\title{
Pharmacological, psychological and non-invasive brain stimulation interventions for preventing depression after stroke (Review)
}

Allida S, Cox KL, Hsieh CF, House A, Hackett ML

Allida S, Cox KL, Hsieh C-F, House A, Hackett ML.

Pharmacological, psychological and non-invasive brain stimulation interventions for preventing depression after stroke.

Cochrane Database of Systematic Reviews 2020, Issue 5. Art. No.: CD003689.

DOI: 10.1002/14651858.CD003689.pub4.

www.cochranelibrary.com

Pharmacological, psychological and non-invasive brain stimulation interventions for preventing depression after 
TABLE OF CONTENTS

HEADER

ABSTRACT

PLAIN LANGUAGE SUMMARY

SUMMARY OF FINDINGS

BACKGROUND

OBJECTIVES

METHODS

Figure 1.

RESULTS

Figure 2.

Figure 3.

DISCUSSION

Figure 4.

AUTHORS' CONCLUSIONS

ACKNOWLEDGEMENTS

REFERENCES

CHARACTERISTICS OF STUDIES

DATA AND ANALYSES

Analysis 1.1. Comparison 1: Pharmacological interventions (antidepressants) versus placebo, Outcome 1: Depression: meeting study criteria for depression at end treatment

Analysis 1.2. Comparison 1: Pharmacological interventions (antidepressants) versus placebo, Outcome 2: Scoring above cutoff points for a depressive disorder at end of treatment

Analysis 1.3. Comparison 1: Pharmacological interventions (antidepressants) versus placebo, Outcome 3: Depression: average change in scores between baseline and end of treatment

Analysis 1.4. Comparison 1: Pharmacological interventions (antidepressants) versus placebo, Outcome 4: Depression: mean scores at end of treatment

Analysis 1.5. Comparison 1: Pharmacological interventions (antidepressants) versus placebo, Outcome 5: Cognition: average change in scores between baseline and end of treatment

Analysis 1.6. Comparison 1: Pharmacological interventions (antidepressants) versus placebo, Outcome 6: Cognition: Mean scores at end of treatment

Analysis 1.7. Comparison 1: Pharmacological interventions (antidepressants) versus placebo, Outcome 7: Activities of daily living: average change in scores between baseline and end of treatment

Analysis 1.8. Comparison 1: Pharmacological interventions (antidepressants) versus placebo, Outcome 8: Activities of daily living: mean scores at end of treatment

Analysis 1.9. Comparison 1: Pharmacological interventions (antidepressants) versus placebo, Outcome 9: Disability: average change in scores between baseline and end of treatment

Analysis 1.10. Comparison 1: Pharmacological interventions (antidepressants) versus placebo, Outcome 10: Disability: mean scores at end of treatment

Analysis 1.11. Comparison 1: Pharmacological interventions (antidepressants) versus placebo, Outcome 11: Disability: moderate to severe disability at end of treatment

Analysis 1.12. Comparison 1: Pharmacological interventions (antidepressants) versus placebo, Outcome 12: Adverse events: death

Analysis 1.13. Comparison 1: Pharmacological interventions (antidepressants) versus placebo, Outcome 13: Adverse events: all

Analysis 1.14. Comparison 1: Pharmacological interventions (antidepressants) versus placebo, Outcome 14: Adverse events: leaving the study early (including death)

Analysis 2.1. Comparison 2: Psychological therapy versus standard care and/or attention control, Outcome 1: Depression: meeting study criteria for depression at end of treatment

Analysis 2.2. Comparison 2: Psychological therapy versus standard care and/or attention control, Outcome 2: Scoring above cut-off points for a depressive disorder at end of treatment

Analysis 2.3. Comparison 2: Psychological therapy versus standard care and/or attention control, Outcome 3: Depression: mean scores at end of treatment

Analysis 2.4. Comparison 2: Psychological therapy versus standard care and/or attention control, Outcome 4: Depression: mean scores at end of follow-up 
Analysis 2.5. Comparison 2: Psychological therapy versus standard care and/or attention control, Outcome 5: Psychological distress: average change in scores between baseline and end of treatment

Analysis 2.6. Comparison 2: Psychological therapy versus standard care and/or attention control, Outcome 6: Psychological distress: mean scores at end of treatment

Analysis 2.7. Comparison 2: Psychological therapy versus standard care and/or attention control, Outcome 7: General Health: average change in scores between baseline and end of treatment

Analysis 2.8. Comparison 2: Psychological therapy versus standard care and/or attention control, Outcome 8: General Health: Mean scores at end of treatment

Analysis 2.9. Comparison 2: Psychological therapy versus standard care and/or attention control, Outcome 9: Social activities: average change in scores between baseline and end of treatment

Analysis 2.10. Comparison 2: Psychological therapy versus standard care and/or attention control, Outcome 10: Social activities: mean scores at end of treatment

Analysis 2.11. Comparison 2: Psychological therapy versus standard care and/or attention control, Outcome 11: Activities of daily living: Average change in scores between baseline and end of treatment

Analysis 2.12. Comparison 2: Psychological therapy versus standard care and/or attention control, Outcome 12: Activities of daily living: mean scores at end of treatment

Analysis 2.13. Comparison 2: Psychological therapy versus standard care and/or attention control, Outcome 13: Activities of daily living: mean scores at end of follow-up

Analysis 2.14. Comparison 2: Psychological therapy versus standard care and/or attention control, Outcome 14: Anxiety: mean scores at end of treatment

Analysis 2.15. Comparison 2: Psychological therapy versus standard care and/or attention control, Outcome 15: Anxiety: mean scores at end of follow-up

Analysis 2.16. Comparison 2: Psychological therapy versus standard care and/or attention control, Outcome 16: Adverse events: death

Analysis 2.17. Comparison 2: Psychological therapy versus standard care and/or attention control, Outcome 17: Adverse events: all

Analysis 2.18. Comparison 2: Psychological therapy versus standard care and/or attention control, Outcome 18: Adverse events: leaving the study early (including death)

Analysis 3.1. Comparison 3: Pharmacological interventions (psychostimulants) versus placebo, Outcome 1: Depression: average change in scores between baseline and end of treatment

Analysis 3.2. Comparison 3: Pharmacological interventions (psychostimulants) versus placebo, Outcome 2: Cognition: average change in scores between baseline and end of treatment

Analysis 3.3. Comparison 3: Pharmacological interventions (psychostimulants) versus placebo, Outcome 3: Disability: average change in scores between baseline and end of treatment

Analysis 3.4. Comparison 3: Pharmacological interventions (psychostimulants) versus placebo, Outcome 4: Adverse events: death

Analysis 3.5. Comparison 3: Pharmacological interventions (psychostimulants) versus placebo, Outcome 5: Adverse events: leaving the study early (including death)

ADDITIONAL TABLES

APPENDICES

WHAT'S NEW

HISTORY

CONTRIBUTIONS OF AUTHORS 
[Intervention Review]

\title{
Pharmacological, psychological and non-invasive brain stimulation interventions for preventing depression after stroke
}

\author{
Sabine Allida ${ }^{1}$, Katherine Laura Cox ${ }^{1}$, Cheng-Fang Hsieh ${ }^{2}$, Allan House ${ }^{3}$, Maree L Hackett 4,5
}

1Mental Health, The George Institute for Global Health, Faculty of Medicine, University of New South Wales, Sydney, Australia. 2Division of Geriatrics and Gerontology, Department of Internal Medicine and Department of Neurology, Kaohsiung Medical University Hospital, Kaohsiung Medical University, Kaohsiung, Taiwan. ${ }^{3}$ Division of Psychological and Social Medicine, Leeds Institute of Health Sciences, University of Leeds, Leeds, UK. ${ }^{4}$ Professor, Program Head, Mental Health, The George Institute for Global Health, Faculty of Medicine, University of New South Wales, Sydney, Australia. ${ }^{5}$ Faculty of Health and Wellbeing, University of Central Lancashire, Preston, Lancashire, UK

Contact address: Maree L Hackett, mhackett@georgeinstitute.org.au.

Editorial group: Cochrane Stroke Group.

Publication status and date: New search for studies and content updated (conclusions changed), published in Issue 5, 2020.

Citation: Allida S, Cox KL, Hsieh C-F, House A, Hackett ML. Pharmacological, psychological and non-invasive brain stimulation interventions for preventing depression after stroke. Cochrane Database of Systematic Reviews 2020, Issue 5. Art. No.: CD003689. DOI: 10.1002/14651858.CD003689.pub4.

Copyright @ 2020 The Cochrane Collaboration. Published by John Wiley \& Sons, Ltd.

\section{A B S T R A C T}

\section{Background}

Depression is an important consequence of stroke that influences recovery yet often is not detected, or is inadequately treated. This is an update and expansion of a Cochrane Review first published in 2004 and previously updated in 2008.

\section{Objectives}

The primary objective is to test the hypothesis that pharmacological, psychological therapy, non-invasive brain stimulation, or combinations of these interventions reduce the incidence of diagnosable depression after stroke. Secondary objectives are to test the hypothesis that pharmacological, psychological therapy, non-invasive brain stimulation or combinations of these interventions reduce levels of depressive symptoms and dependency, and improve physical functioning after stroke. We also aim to determine the safety of, and adherence to, the interventions.

\section{Search methods}

We searched the Specialised Register of Cochrane Stroke and the Cochrane Depression Anxiety and Neurosis (last searched August 2018). In addition, we searched the following databases; Cochrane Central Register of Controlled Trials, CENTRAL (the Cochrane Library, 2018, Issue 8), MEDLINE (1966 to August 2018), Embase (1980 to August 2018), PsycINFO (1967 to August 2018), CINAHL (1982 to August 2018) and three Web of Science indexes (2002 to August 2018). We also searched reference lists, clinical trial registers (World Health Organization International Clinical Trials Registry Platform (WHO ICTRP); to August 2018 and ClinicalTrials.gov; to August 2018), conference proceedings; we also contacted study authors.

\section{Selection criteria}

Randomised controlled trials (RCTs) comparing: 1) pharmacological interventions with placebo; 2) one of various forms of psychological therapy with usual care and/or attention control; 3) one of various forms of non-invasive brain stimulation with sham stimulation or usual care; 4) a pharmacological intervention and one of various forms of psychological therapy with a pharmacological intervention and usual care and/or attention control; 5) non-invasive brain stimulation and pharmacological intervention with a pharmacological intervention and sham stimulation or usual care; 6) pharmacological intervention and one of various forms of psychological therapy with placebo and psychological therapy; 7) pharmacological intervention and non-invasive brain stimulation with placebo plus non-invasive brain 
stimulation; 8) non-invasive brain stimulation and one of various forms of psychological therapy versus non-invasive brain stimulation plus usual care and/or attention control; and 9) non-invasive brain stimulation and one of various forms of psychological therapy versus sham brain stimulation or usual care plus psychological therapy, with the intention of preventing depression after stroke.

\section{Data collection and analysis}

Review authors independently selected studies, assessed risk of bias, and extracted data from all included studies. We calculated mean difference (MD) or standardised mean difference (SMD) for continuous data and risk ratio (RR) for dichotomous data with $95 \%$ confidence intervals (Cls). We assessed heterogeneity using the $\mathrm{I}^{2}$ statistic and assessed the certainty of evidence using GRADE.

\section{Main results}

We included 19 RCTs (21 interventions), with 1771 participants in the review. Data were available for 12 pharmacological trials (14 interventions) and seven psychological trials. There were no trials of non-invasive brain stimulation compared with sham stimulation or usual care, a combination of pharmacological intervention and one of various forms of psychological therapy with placebo and psychological therapy, or a combination of non-invasive brain stimulation and a pharmacological intervention with a pharmacological intervention and sham stimulation or usual care to prevent depression after stroke. Treatment effects were observed on the primary outcome of meeting the study criteria for depression at the end of treatment: there is very low-certainty evidence from eight trials (nine interventions) that pharmacological interventions decrease the number of people meeting the study criteria for depression (RR $0.50,95 \%$ $\mathrm{Cl} 0.37$ to $0.68 ; 734$ participants) compared to placebo. There is very low-certainty evidence from two trials that psychological interventions reduce the proportion of people meeting the study criteria for depression (RR $0.68,95 \% \mathrm{Cl} 0.49$ to $0.94,607$ participants) compared to usual care and/or attention control.

Eight trials (nine interventions) found no difference in death and other adverse events between pharmacological intervention and placebo groups ( $\mathrm{RR} 1.25,95 \% \mathrm{Cl} 0.32$ to 4.91; 496 participants) based on very low-certainty evidence. Five trials found no difference in psychological intervention and usual care and/or attention control groups for death and other adverse events (RR $1.18,95 \% \mathrm{Cl} 0.73$ to $1.91 ; 975$ participants) based on very low-certainty evidence.

\section{Authors' conclusions}

The available evidence suggests that pharmacological interventions and psychological therapy may prevent depression and improve mood after stroke. However, there is very low certainty in these conclusions because of the very low-certainty evidence. More trials are required before reliable recommendations can be made about the routine use of such treatments after stroke.

\section{PLAIN LANGUAGE SUMMARY}

\section{Interventions for preventing depression after stroke}

\section{Review question}

Do pharmacological, psychological, non-invasive brain stimulation or a combination of these interventions prevent depression and improve outcomes after stroke?

\section{Background}

The role of interventions for preventing depression after stroke is unclear. Depression is a common and important complication of stroke that is often missed or poorly managed. Little is known about whether prevention strategies started early after stroke will reduce the risk of depression and improve recovery for those not depressed at assessment.

\section{Search date}

We identified trials using searches conducted on 13 August 2018.

\section{Study characteristics}

We included trials which reported on the use of pharmacological and psychological interventions to prevent depression after stroke. Average age of participants ranged from 55 to 73 years. Trials were from Asia (3), Europe (8), North America (5), and Australia (3).

\section{Key results}

We included 19 trials (12 pharmacological and seven psychological) involving 1771 participants. Outcome information was available for nine pharmacological and two psychological trials, which suggested that these treatments might reduce the risk of developing depression. A smaller number of studies (eight pharmacological and five psychological studies) found no increase in death or adverse events.

\section{Certainty of evidence}

We rated the certainty of evidence as very low due to limitations in study design. 


\section{Conclusion}

Our ability to generalise these findings to all stroke survivors is limited due to the small proportion of survivors who were eligible to participate in these clinical trials. More well-designed clinical trials are needed that test practical interventions for preventing depression across all stroke survivors. 
SUMMARY OF FINDINGS

Summary of findings 1. Pharmacological interventions (antidepressants) compared to placebo for preventing depression after stroke

Pharmacological interventions (antidepressants) compared to placebo for preventing depression after stroke

Patient or population: people with stroke

Setting: hospital and community

Intervention: pharmacological interventions (antidepressants)

Comparison: placebo

\begin{tabular}{|c|c|c|c|c|c|c|}
\hline \multirow[t]{2}{*}{ Outcomes } & \multicolumn{2}{|c|}{ Anticipated absolute effects ${ }^{*}(95 \% \mathrm{CI})$} & \multirow{2}{*}{$\begin{array}{l}\text { Relative } \\
\text { effect } \\
(95 \% \mathrm{CI})\end{array}$} & \multirow{2}{*}{$\begin{array}{l}\text { No of par- } \\
\text { ticipants } \\
\text { (studies) }\end{array}$} & \multirow{2}{*}{$\begin{array}{l}\text { Certainty } \\
\text { of the evi- } \\
\text { dence } \\
\text { (GRADE) }\end{array}$} & \multirow[t]{2}{*}{ Comments } \\
\hline & Risk with placebo & $\begin{array}{l}\text { Risk with pharmacological } \\
\text { interventions (antidepres- } \\
\text { sants) }\end{array}$ & & & & \\
\hline \multirow{2}{*}{$\begin{array}{l}\text { Depression: meeting study crite- } \\
\text { ria for depression at end treatment } \\
\text { (Analysis 1.1) }\end{array}$} & \multicolumn{2}{|l|}{ Study population } & \multirow{2}{*}{$\begin{array}{l}\text { RR } 0.50 \\
(0.37 \text { to } \\
0.68)\end{array}$} & \multirow{2}{*}{$\begin{array}{l}734 \\
(9 \text { RCTs) }\end{array}$} & \multirow{2}{*}{$\begin{array}{l}\oplus \ominus \ominus \ominus \\
\text { Very } \\
\text { lowa,b }\end{array}$} & \\
\hline & 250 per 1000 & $\begin{array}{l}125 \text { per } 1000 \\
(92.5 \text { to } 170)\end{array}$ & & & & \\
\hline $\begin{array}{l}\text { Scoring above cut-off points for a de- } \\
\text { pressive disorder at end of treatment } \\
\text { (Analysis 1.2) }\end{array}$ & - & & - & (0 RCTs) & - & No data available. \\
\hline \multicolumn{2}{|c|}{ Depression: mean scores at end of treatment (Analysis 1.4) } & $\begin{array}{l}\text { MD } 0.59 \text { higher } \\
\text { (1.46 lower to } 2.63 \text { higher) }\end{array}$ & - & $\begin{array}{l}100 \\
(4 \mathrm{RCTS})\end{array}$ & $\begin{array}{l}\oplus \ominus \ominus \ominus \\
\text { Very } \\
\text { lowa,c }\end{array}$ & $\begin{array}{l}\text { HDRS }(\text { high score = more de- } \\
\text { pressed) }\end{array}$ \\
\hline \multicolumn{2}{|c|}{$\begin{array}{l}\text { Activities of daily living: mean scores at end of treatment } \\
\text { (Analysis 1.8) }\end{array}$} & $\begin{array}{l}\text { MD } 3.86 \text { lower } \\
\text { (9.48 lower to } 1.77 \text { higher) }\end{array}$ & - & $\begin{array}{l}116 \\
\text { (3 RCTs) }\end{array}$ & $\begin{array}{l}\oplus \Theta \Theta \Theta \\
\text { Very } \\
\text { lowa,c }\end{array}$ & $\begin{array}{l}\text { Barthel Index (high score = more } \\
\text { dependent) }\end{array}$ \\
\hline \multicolumn{2}{|c|}{ Disability: mean scores at end of treatment (Analysis 1.10) } & - & - & $\begin{array}{l}204 \\
\text { (4 studies) }\end{array}$ & - & $\begin{array}{l}\text { No totals. } \\
\text { Hemispheric Stroke Scale Total } \\
\text { Score (high score = more neuro- } \\
\text { logical deficit) }\end{array}$ \\
\hline
\end{tabular}




\begin{tabular}{|c|c|c|c|c|c|c|}
\hline & & & & & & $\begin{array}{l}\text { Johns Hopkins Functioning In- } \\
\text { ventory (high score = less func- } \\
\text { tion) }\end{array}$ \\
\hline \multirow{2}{*}{$\begin{array}{l}\text { Adverse events: death - at end of } \\
\text { treatment Analysis } 1.12\end{array}$} & \multicolumn{2}{|c|}{ Study population } & \multirow{2}{*}{$\begin{array}{l}\text { RR } 1.25 \\
(0.32 \text { to } \\
4.91)\end{array}$} & \multirow{2}{*}{$\begin{array}{l}496 \\
\text { (9 RCTs) }\end{array}$} & \multirow{2}{*}{$\begin{array}{l}\oplus \ominus \ominus \ominus \\
\text { Very } \\
\text { lowa,c }\end{array}$} & \\
\hline & 0 per 1000 & $\begin{array}{l}0 \text { per } 1000 \\
(0 \text { to } 0)\end{array}$ & & & & \\
\hline
\end{tabular}

${ }^{*}$ The risk in the intervention group (and its $95 \%$ confidence interval) is based on the assumed risk in the comparison group and the relative effect of the intervention (and its $95 \% \mathrm{Cl})$.

CI: confidence interval; HDRS: Hamilton Depression Rating Scale; MMSE: Mini Mental State Examination; MD: mean difference; RCT: randomised controlled trial; RR: risk ratio

\section{GRADE Working Group grades of evidence}

High certainty: we are very confident that the true effect lies close to that of the estimate of the effect.

Moderate certainty: we are moderately confident in the effect estimate; the true effect is likely to be close to the estimate of the effect, but there is a possibility that it is substantially different.

Low certainty: our confidence in the effect estimate is limited; the true effect may be substantially different from the estimate of the effect.

Very low certainty: we have very little confidence in the effect estimate; the true effect is likely to be substantially different from the estimate of effect.

aWe downgraded the certainty of evidence as the studies were rated as unclear or high risk in multiple risk of bias domains.

$b$ We downgraded the certainty of evidence because the confidence intervals were wide.

cWe downgraded the certainty of evidence because the confidence intervals were very wide.

\section{Summary of findings 2. Psychological therapy compared to usual care and/or attention control for preventing depression after stroke}

\section{Psychological therapy compared to usual care and/or attention control for preventing depression after stroke}

Patient or population: people with stroke

Setting: hospital and community

Intervention psychological therapy

Comparison: usual care and/or attention control

\begin{tabular}{|c|c|c|c|c|c|c|}
\hline \multirow[t]{2}{*}{ Outcomes } & \multicolumn{2}{|c|}{ Anticipated absolute effects* $(95 \% \mathrm{Cl})$} & \multirow{2}{*}{$\begin{array}{l}\text { Relative } \\
\text { effect } \\
(95 \% \mathrm{CI})\end{array}$} & \multirow{2}{*}{$\begin{array}{l}\text { No of par- } \\
\text { ticipants } \\
\text { (studies) }\end{array}$} & \multirow{2}{*}{$\begin{array}{l}\text { Certain- } \\
\text { ty of } \\
\text { the evi- } \\
\text { dence } \\
\text { (GRADE) }\end{array}$} & \multirow[t]{2}{*}{ Comments } \\
\hline & $\begin{array}{l}\text { Risk with usual care and/ } \\
\text { or attention control }\end{array}$ & $\begin{array}{l}\text { Risk with psycho- } \\
\text { logical therapy }\end{array}$ & & & & \\
\hline $\begin{array}{l}\text { Depression: meeting study criteria for de- } \\
\text { pression at end of treatment (Analysis } 2.1 \text { ) }\end{array}$ & Study population & & RR 0.68 & $\begin{array}{l}607 \\
(2 \mathrm{RCTs})\end{array}$ & $\oplus \odot \ominus \ominus$ & \\
\hline
\end{tabular}




\begin{tabular}{|c|c|c|c|c|c|c|c|}
\hline 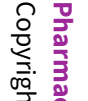 & & 296 per 1000 & $\begin{array}{l}201 \text { per } 1000 \\
\text { (145 to } 278)\end{array}$ & $\begin{array}{l}(0.49 \text { to } \\
0.94)\end{array}$ & & $\begin{array}{l}\text { Very } \\
\text { lowa,b }\end{array}$ & \\
\hline 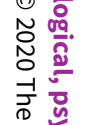 & $\begin{array}{l}\text { Scoring above cut-off points for a depres- } \\
\text { sive disorder at end of treatment (Analysis } \\
2.2 \text { ) }\end{array}$ & - & - & - & (0 RCTs) & - & No data available \\
\hline 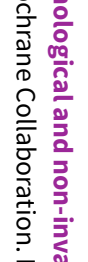 & \multicolumn{2}{|c|}{ Depression: mean scores at end of treatment (Analysis 2.3) } & - & - & $\begin{array}{l}132 \\
\text { (2 studies) }\end{array}$ & - & $\begin{array}{l}\text { No totals } \\
\text { HDRS (high score = more de- } \\
\text { pressed) } \\
\text { MADRS (high score = more de- } \\
\text { pressed) }\end{array}$ \\
\hline 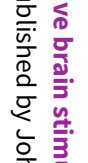 & \multicolumn{2}{|c|}{ Psychological distress: mean scores at end of treatment (Analysis 2.6) } & - & - & $\begin{array}{l}450 \\
(1 \mathrm{RCT})\end{array}$ & & $\begin{array}{l}\text { No totals } \\
\text { GHQ-28 (high score = greater psy- } \\
\text { chological distress) }\end{array}$ \\
\hline 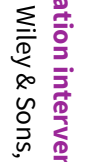 & \multicolumn{2}{|c|}{ General Health: mean scores at end of treatment (Analysis 2.8) } & $\begin{array}{l}\text { MD } 4.60 \text { higher } \\
\text { ( } 21.25 \text { lower to } \\
30.45 \text { higher) }\end{array}$ & - & $\begin{array}{l}240 \\
\text { (1 study) }\end{array}$ & - & $\begin{array}{l}\text { No totals } \\
\text { Nottingham Health Profile (high } \\
\text { score = better health) }\end{array}$ \\
\hline $\begin{array}{l}? \\
\stackrel{0}{0} \\
\stackrel{0}{0} \\
\stackrel{0}{0}\end{array}$ & \multicolumn{2}{|c|}{ Social activities: mean scores at end of treatment (Analysis 2.10) } & $\begin{array}{l}\text { MD } 0.39 \text { lower } \\
\text { (3.81 lower to } 3.03 \\
\text { higher) }\end{array}$ & - & $\begin{array}{l}690 \\
(2 \mathrm{RCTs})\end{array}$ & $\begin{array}{l}\oplus \Theta \Theta \odot \\
\text { Very } \\
\text { lowa,c,d }\end{array}$ & $\begin{array}{l}\text { Frenchay Activities Index (high } \\
\text { score = better level of activity) }\end{array}$ \\
\hline 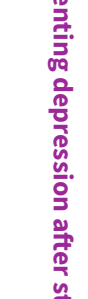 & \multicolumn{2}{|c|}{ Activities of daily living: mean scores at end of treatment (Analysis 2.12) } & - & - & $\begin{array}{l}879 \\
\text { (4 studies) }\end{array}$ & - & $\begin{array}{l}\text { No totals } \\
\text { Barthel Index (high score = more } \\
\text { dependent) } \\
\text { Nottingham Extended Activities of } \\
\text { daily living (high score = more inde- } \\
\text { pendent) }\end{array}$ \\
\hline 要 & \multirow{2}{*}{$\begin{array}{l}\text { Adverse events: death - at end of treat- } \\
\text { ment (Analysis 2.16) }\end{array}$} & Study population & & \multirow{2}{*}{$\begin{array}{l}\text { RR } 1.18 \\
(0.73 \text { to } \\
1.91)\end{array}$} & \multirow{2}{*}{$\begin{array}{l}975 \\
\text { (5 RCTs) }\end{array}$} & \multirow{2}{*}{$\begin{array}{l}\oplus \ominus \Theta \Theta \\
\text { Very } \\
\text { lowa,c }\end{array}$} & \\
\hline 通 & & 42 per 1000 & $\begin{array}{l}49 \text { per } 1000 \\
\text { (30 to } 79)\end{array}$ & & & & \\
\hline
\end{tabular}

${ }^{*}$ The risk in the intervention group (and its $95 \%$ confidence interval) is based on the assumed risk in the comparison group and the relative effect of the intervention (and its $95 \% \mathrm{Cl}$ ). 


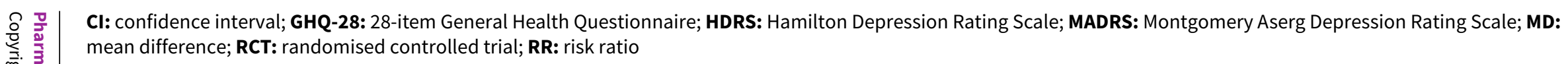

\title{
GRADE Working Group grades of evidence
}

High certainty: we are very confident that the true effect lies close to that of the estimate of the effect.

Moderate certainty: we are moderately confident in the effect estimate; the true effect is likely to be close to the estimate of the effect, but there is a possibility that it is substantially different.

Low certainty: our confidence in the effect estimate is limited; the true effect may be substantially different from the estimate of the effect.

Very low certainty: we have very little confidence in the effect estimate; the true effect is likely to be substantially different from the estimate of effect.

aWe downgraded the certainty of evidence as the studies were rated high risk in multiple risk of bias domains.

$b$ We downgraded the certainty of evidence because the confidence intervals were wide.

cWe downgraded the certainty of evidence because the confidence intervals were very wide.

$d$ We downgraded the certainty of evidence because of substantial heterogeneity observed $(I=80 \%)$. 


\section{B A C K G R O U N D}

\section{Description of the condition}

Depressive and anxiety disorders are important sequelae of stroke, occurring in up to half of people in the first year after onset, although estimates differ between studies due to varying definitions, populations, exclusion criteria, and the timing of assessments (Ayerbe 2013; Hackett 2014). Although there is much controversy surrounding stroke-associated depression as a specific type of depressive syndrome, the condition may impede rehabilitation (Parikh 1990; Sinyor 1986), by impairing physical and cognitive function (Robinson 1986), and contributing to stress on carers (Anderson 1995). Furthermore, depression following stroke may also be associated with an increased risk of death (House 2001; Morris 1993), including death by suicide (Stenager 1998). Depressive illness among older people, in general, is associated with greater morbidity and dependency, higher use of drugs and alcohol, increased use of healthcare resources, and poor compliance with treatment of co-morbid conditions (Katona 1995).

\section{Description of the intervention}

We considered three broad interventions in this review.

- Pharmacological interventions designed to prevent depression: there are several classes of relevant pharmacological agents including selective serotonin reuptake inhibitors (SSRIs) (e.g. le fluvoxamine, fluoxetine, sertraline, citalopram, and paroxetine), serotonin-norepinephrine reuptake inhibitors (SNRIs) (e.g. venlafaxine, milnacipran, sibutramine), monoamine oxidase inhibitors (MAOIs) (e.g. moclobemide), tricyclic antidepressants (TCAs) (e.g. nortriptyline, imipramine, and clomipramine), and other antidepressant medications including psychostimulants (e.g. methylphenidate), mood stabilisers (e.g. lithium), or benzodiazepines.

- One of various forms of psychological intervention (talking therapy) designed to prevent depression: as there are many potential therapies we included any psychological intervention that involved direct person-professional interaction. The content of the interaction could vary from counselling to specific psychotherapy provided it was directed at helping people develop their social problem-solving skills and adjustment to the emotional impact of stroke. All interventions had to have a psychological component - talking, listening, support, advice; be based on a theory of talking therapy; be structured and time-tabled as a talking therapy; and be delivered by somebody with some explicitly stated training and supervision in therapies. The person-professional interaction could take place in person, via telephone or other media. We did not include web-based interventions even if mediated by a health professional. We did not include interventions based upon self-management or supported self-management.

- Non-invasive brain stimulation designed to prevent depression: electroconvulsive therapy (ECT) involves the brief passage of an electrical current through the brain via electrodes applied to the scalp to induce a generalised seizure (i.e. a fit or convulsion). The seizure comprises two components: a central element, the ictus involving depolarisation (i.e. discharge of neurotransmitter chemicals) of brain cells, and a peripheral element of convulsive, jerking movements of the body, although this is now modified due to use of a short-acting anaesthetic and muscle relaxant, as part of what is called modified ECT. Modified ECT replaced the initial crude equipment and techniques of unmodified ECT from the mid-1950s. The seizure is detected by electrodes placed on the scalp to monitor brain electrical activity (i.e. EEG). The ECT electrodes can be placed on both sides of the head (bilateral placement), or on one side, usually the right side of the head (unilateral placement). The passage of an electrical current through the skull to the brain is necessary to trigger a seizure. In this update, we expanded the review to include other non-invasive brain stimulation techniques such as 1) transcranial magnetic stimulation or repetitive transcranial magnetic stimulation (TMS or rTMS, where a magnetic 'coil' is placed near the head of the person receiving the treatment without making physical contact); 2) transcranial direct current stimulation (tDCS, where a constant, low current is delivered directly to the brain area of interest via small electrodes); 3) cranial electrotherapy stimulation (CES, where a small, pulsed electric current is applied across a person's head), and 4) magnetic seizure therapy (MST), a type of convulsive therapy that involves replacing the electrical stimulation used in ECT with a rapidly alternating strong magnetic stimulation.

We further considered these combinations of the three broad interventions.

- Pharmacological intervention and one of various forms of psychological therapy with a pharmacological intervention and usual care and/or attention control.

- Pharmacological intervention and one of various forms of psychological therapy with placebo and psychological therapy.

- Pharmacological intervention and non-invasive brain stimulation with a pharmacological intervention and sham stimulation or usual care.

- Pharmacological intervention and non-invasive brain stimulation with placebo plus non-invasive brain stimulation.

- Non-invasive brain stimulation and one of various forms of psychological therapy versus non-invasive brain stimulation plus usual care and/or attention control.

- Non-invasive brain stimulation and one of various forms of psychological therapy versus sham brain stimulation or usual care plus psychological therapy.

\section{How the intervention might work}

Pharmacological interventions are thought to alter the synaptic transmission process in the brain to increase neurotransmission, for example serotonin reuptake SSRIs are intended to block the transport of serotonin, SNRIs are designed to increase the levels of serotonin and norepinephrine, and TCAs are designed to block the reuptake of norepinephrine.

Psychological interventions focus on changing thought, emotional, behavioural, and relationship patterns. During psychological interventions, trained therapists work with individuals to help them see patterns in their thoughts, emotions, behaviours, or relationships that may be problematic. The therapist's role is to help a person understand the patterns and assist them in developing ways to overcome them.

During modified ECT a small amount of electric current is passed briefly across the brain to cause an artificial epileptic fit that affects the entire brain. Repeated ECT is believed to alter chemical pathways in the brain that are responsible for depression. The 
exact mechanism of action of TMS, rTMS, tDCS, and CES remains unclear. They are thought to induce intracerebral current flow, increase or decrease neuronal excitability and/or activate nerve cells in the specific area being stimulated. MST involves replacing the electrical stimulation used in ECT with a magnetic stimulus, which is purported to produce similar clinical effects but without the cognitive side effects.

\section{Why it is important to do this review}

Although depression may influence recovery and outcome following stroke, many, perhaps most, people do not receive effective treatment because their mood disorder is undiagnosed or inadequately treated (Ebrahim 1987; Hackett 2005a; House 1989). This is due in part to the problems with the diagnosis of a significant mood state among older people with disability. Even in otherwise healthy individuals, the assessment of abnormal mood is fraught with difficulty. Information from the general population suggests that, despite the severity and possible complications, only half of those with depression will seek professional help (WHO 2000). This may be due to the stigma associated with a diagnosis, people not realising they are unwell, or feeling their condition is beyond help, a natural part of ageing or a consequence of stroke. Given the problems inherent with diagnosis, that people are at high risk of developing depression after stroke, and the uncertainty about the balance of benefit and risks of treatment, there is interest in therapies commenced early after the onset of stroke that prevent abnormal mood and improve outcome. We undertook a systematic review of all randomised controlled trials (RCTs), both published and unpublished, of pharmacological agents, psychological interventions, non-invasive brain stimulation, or their combination for the prevention of depression associated with stroke. This is an update of a Cochrane Review first published in 2004 (Anderson 2004), and last updated in 2008 (Hackett 2008).

\section{OB JECTIVES}

\section{Primary objective}

- To determine whether pharmacological therapy, psychological therapy, non-invasive brain stimulation or combinations of these interventions prevent the incidence of diagnosable depression after stroke.

\section{Secondary objectives}

- To determine whether pharmacological therapy, psychological therapy, non-invasive brain stimulation or combinations of these interventions reduce levels of depressive symptoms, improve physical and neurological function and health-related quality of life, and reduce dependency after stroke.

- To assess the safety of, and adherence to such treatments.

\section{ME T HO D S}

\section{Criteria for considering studies for this review}

\section{Types of studies}

We restricted the review to all relevant randomised controlled trials (RCTs). There was no restriction on eligibility of RCTs on the basis of language, sample size, duration of follow-up, or publication status. Trials that met all the inclusion criteria, but in which no outcome data were available (either from the report of the trial or from the authors), could not contribute meaningfully to a pooled estimate of effect. These trials were regarded as 'dropouts' rather than ineligible and we have listed them in an additional table to indicate that they have not been overlooked (Table 1).

\section{Types of participants}

We included all participants with a confirmed history of stroke where there was an explicit intention to provide an intervention to prevent depression associated with stroke. Stroke was defined according to clinical criteria. The criteria included cerebral infarction, intracerebral haemorrhage, and uncertain pathological subtypes, but excluded studies of subarachnoid haemorrhage (SAH) only, which has a different natural history and management strategy to other stroke subtypes. We included studies with small numbers of participants with SAH. We excluded trials that included mixed populations (such as stroke and head injury or other central nervous system disorders) unless separate results for participants with stroke were identified. We excluded participants with a diagnosed depressive disorder or a mood score above the standard cut-off score for depressive disorder at baseline, but included them in a review of pharmacological, psychological and noninvasive brain stimulation interventions for treating depression after stroke (Allida 2020). We excluded participants if they were treated primarily for a stroke-associated pain syndrome or other physical disorder, even if depression was measured as a secondary outcome.

\section{Types of interventions}

We included the following interventions.

- Comparison between a pharmacological intervention and placebo for the prevention of depression after stroke. Specific pharmacological agents included tricyclic antidepressants (e.g. nortriptyline, imipramine, and clomipramine), selective serotonin reuptake inhibitors (SSRIs) (e.g. fluvoxamine, fluoxetine, sertraline, and paroxetine), monoamine oxidase inhibitors (MAOIs) (e.g. moclobemide), and other antidepressant medications. We included psychostimulants (e.g. methylphenidate), mood stabilisers (e.g. lithium), benzodiazepines, and combined preparations, but analysed these separately.

- Comparison between psychological therapy and usual care (or attention control) for the prevention of depression after stroke. We included any psychological therapy that involved direct person-professional interaction. The content of the interaction could vary from counselling to specific psychological therapy, provided it was directed at helping people develop their social problem-solving skills and adjustment to the emotional impact of stroke. All interventions had to have a psychological component, such as talking, listening, support, advice; be based on a theory of talking therapy; be structured and time-tabled as a talking therapy; and be delivered by somebody with some explicitly stated training and supervision in therapies.

- Comparison between non-invasive brain stimulation and sham stimulation or usual care for the prevention of depression associated with stroke. We found no trials of non-invasive brain stimulation interventions. Any future trials will be included but analysed separately.

Alternatively, we included their combinations. 
- Pharmacological intervention and one of various forms of psychological therapy versus pharmacological intervention plus usual care and/or attention control.

- Pharmacological intervention and one of various forms of psychological therapy versus placebo plus psychological therapy.

- Pharmacological intervention and non-invasive brain stimulation versus pharmacological intervention plus sham stimulation or usual care.

- Pharmacological intervention and non-invasive brain stimulation versus placebo plus non-invasive brain stimulation.

- Non-invasive brain stimulation and one of various forms of psychological therapy versus non-invasive brain stimulation plus usual care and/or attention control.

- Non-invasive brain stimulation and one of various forms of psychological therapy versus sham brain stimulation or usual care plus psychological therapy.

Exclusions included the following.

- Interventions with an agent or therapy that was being primarily evaluated for other reasons (for example, to improve physical function, provide neuroprotection, or to facilitate neuroregeneration) with a mood endpoint.

- Interventions with the sole purpose of educating or providing information.

- Occupational therapy (including leisure therapy and other rehabilitation services).

- Acupuncture or electro-acupuncture.

- Herbal medicines.

- Interventions which involved visits from stroke support workers, unless there was a clearly defined psychological component.

Attention control in psychological therapy trials can include nonspecific interventions such as relaxation classes, or follow-up with a clinician who has no psychological training.

\section{Types of outcome measures}

\section{Primary outcomes}

The primary analyses focus on the proportion of people who met the diagnostic categories of depression that were applied by the authors of the trial, at the end of the treatment period. These included:

- meeting the criteria for depression, dysthymia or minor depression as defined by the American Psychiatric Association Diagnostic and Statistical Manual of Mental Disorders (DSM-IIIR, DSM-IV, DSM V; APA 1987; APA 1994; APA 2013) or similar standard diagnostic criteria;

- scoring above cut-off points for a depressive disorder, as defined by symptom scores on standard mood rating scales.

\section{Secondary outcomes}

The secondary outcomes were:

- depression score, as measured on scales such as the Hamilton Depression Rating Scale (HDRS, Hamilton 1960), Montgomery Åsberg Depression Rating Scale (MADRS, Montgomery 1979), Geriatric Depression Scale (GDS, Gompertz 1993), Beck Depression Inventory (BDI, Beck 1961), and Hospital Anxiety and
Depression Scale (HADS Depression sub-scale, Zigmond 1983) at the end of treatment/follow-up;

- psychological distress, as measured on composite scales such as the General Health Questionnaire (GHQ, Goldberg 1972);

- general health, as measured on composite scales such as the Nottingham Health Profile (NHP, Hunt 1986);

- cognition, as measured on scales such as the Mini-Mental State Examination (MMSE, Folstein 1975);

- social activities, as measured on scales such as the Frenchay Activities Index (FAl, Wade 1985);

- activities of daily living, as measured on scales such as the Barthel Index (BI, Mahoney 1965);

- disability, as measured on scales such as the Hemispheric Stroke Scale (HSS, Adams 1990);

- disadvantages of treatment, recorded as adverse events; grouped by death, all, and leaving the study early (including death).

We examined the reason for participants withdrawing from the studies as a marker of acceptance.

We have identified additional outcomes, where measured, for use in further reviews.

- Anxiety, as measured on scales such as the Hamilton Anxiety Scale, Beck Anxiety Inventory, and the Hospital Anxiety and Depression Scale (HADS Anxiety sub-scale, Zigmond 1983).

\section{Search methods for identification of studies}

This review is an update of a previously published Cochrane Review updated in 2008 (Hackett 2008; Appendix 1). The first published review was in 2004 (Anderson 2004). For this update, we searched all databases from inception until August 2018. We searched for relevant trials in all languages and arranged for translation of trial reports when necessary.

\section{Specialised Register of Cochrane Stroke}

See the methods for the Cochrane Stroke Group Specialised register. The Cochrane Stroke Group Information Specialist searched the Specialised Register of Cochrane Stroke on 13 August 2018.

\section{Electronic searches}

We searched the following bibliographic databases.

- Cochrane Depression Anxiety and Neurosis Trials Register (last searched August 2018)

- Cochrane Central Register of Controlled Trials (CENTRAL; 2018, Issue 8), in the Cochrane Library (Appendix 2)

- MEDLINE (OVID):1966 to August 2018 (Appendix 3)

- Embase (OVID): 1980 to August 2018 (Appendix 4)

- PsycINFO (OVID): 1967 to August 2018 (Appendix 5)

- Cumulative Index to Nursing and Allied Health Literature, CINAHL (EBSCO): 1982 to August 2018 (Appendix 6)

- Science Citation Index - Expanded (SCI-EXPANDED), Social Sciences Citation Index (SSCI), and Arts \& Humanities Citation Index (A\&HCI) in Web of Science (ISI): 2002 to August 2018 (Appendix 7)

Pharmacological, psychological and non-invasive brain stimulation interventions for preventing depression after stroke (Review) 
Biological Abstracts has now been superseded by ISI Web of Science, which includes the Arts and Humanities Index. Several databases/citation indexes (Applied Science and Technology Plus; Biological Abstracts; BIOSIS Previews; General Science Plus; Dissertations and Theses) listed in Appendix 1 were not used for this update.

We also searched the following ongoing trials registers and registries using 'stroke' or 'brain infarction' or 'depression' or 'low mood' from inception to August 2018.

- US National Institutes of Health Ongoing Trials Register ClinicalTrials.gov (www.clinicaltrials.gov)

- World Health Organization International Clinical Trials Registry Platform ( WHO ICTRP) (www.who.int/ictrp/en/)

\section{Searching other resources}

In addition, we also searched abstracts and conference proceedings from the following international conferences for relevant studies.

- European Stroke Organisation Conference (2015 to 2018)

- Stroke Society of Australasia Annual Scientific Meetings (2008 to 2018)

- World Stroke Congress (2000 to 2016)

- Asia Pacific Stroke Conference (2011 to 2017)

The full search strategies for other resources are presented in Appendix 8.

\section{Personal communication}

We contacted the study authors for information on ongoing and 'dropout' trials or to request additional study data and in some instances, additional analyses.

\section{Reference lists}

We searched the reference lists of relevant trials, systematic reviews and reviewed chapters in books on the prevention and treatment of depression and management of stroke, including but not limited to, reviews of the management of stroke, books specifically directed at the treatment or prevention of depression, and those on stroke and old age.

\section{Data collection and analysis}

\section{Selection of studies}

Two review authors $(\mathrm{SA}, \mathrm{KC})$ reviewed all citations for this update and discarded those that were irrelevant, based on the title of the publication and its abstract. In the presence of any suggestion that an article was possibly relevant, we retrieved the full-length article for further assessment. Two review authors (SA, KC) independently selected the trials for inclusion in the review from the culled citation list. Potentially relevant Chinese language articles were translated by another study author (C-FH). We resolved disagreements by discussion, and $\mathrm{MH}$ and $\mathrm{AH}$ confirmed the final list and adjudicated any persisting differences of opinion. The selection process is presented in a PRISMA flow diagram (Figure 1). We listed the included studies under Characteristics of included studies, and studies that we ultimately excluded under Characteristics of excluded studies and provided the primary reasons for exclusion. 
Figure 1. Study flow diagram.

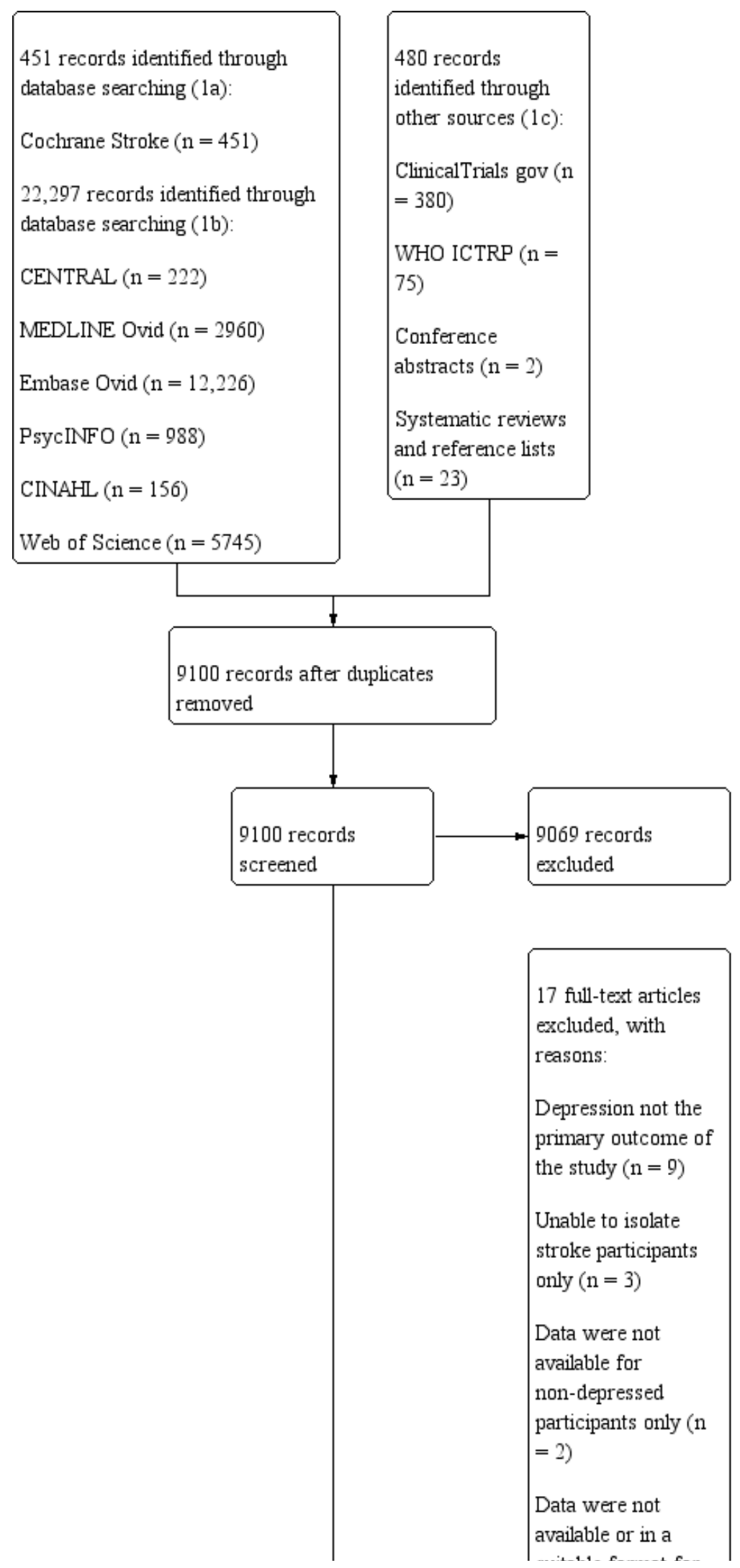


Figure 1. (Continued)

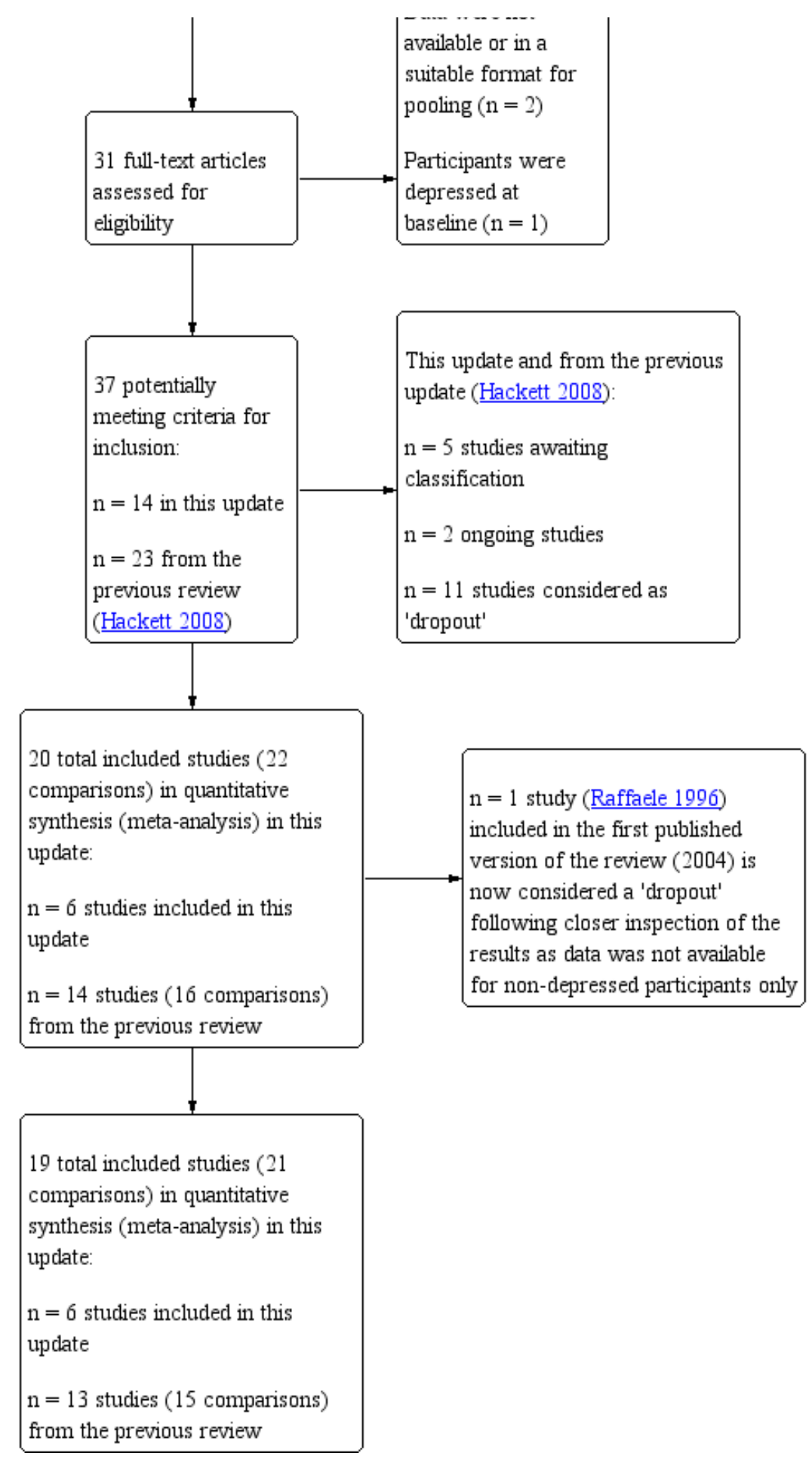

\section{Data extraction and management}

Four review authors (SA, KC, C-FH, MH) independently extracted the study characteristics and outcome data from studies included in this update, on specially designed forms. We cross-checked and entered the data into Review Manager 5 (Review Manager 2014).We resolved disagreements by discussion or through consultation with two other review authors $(\mathrm{AH}$ or $\mathrm{MH})$. We obtained missing information from the study authors when possible. Information on funding sources are mentioned in the notes section of the Characteristics of included studies table.

We collected data on:

- the report: author, year, and source of publication; 
- the study: sample characteristics, social demography, definition and criteria used for depression;

- the participants: stroke sequence (first ever versus recurrent), social situation, time elapsed since stroke onset, history of psychiatric illness, current neurological status, current treatment for depression, and a history of coronary artery disease;

- the research design and features: sampling mechanism, treatment assignment mechanism, adherence, non-response, and length of follow-up;

- the intervention: type, duration, dose, timing, and mode of delivery;

- the effect size: sample size, nature of outcome, estimate and standard error on $\mathrm{x} d \mathrm{y}=\mathrm{SD}$.

To allow for intention-to-treat (ITT) analysis, we sought the data irrespective of adherence, fidelity of the intervention to the protocol, and regardless of whether the participants were subsequently deemed ineligible or otherwise excluded from treatment or follow-up. Where study authors used multiple measures to assess depression, we extracted data from the measure the study authors stated was used to assess the primary outcome. For measures assessing secondary outcomes, we extracted data from the most commonly used measure. Where data for the same trial endpoint were conflicting across multiple publications, we extracted data from the first publication reporting data for that outcome. We checked all the extracted data for agreement between review authors. We obtained missing information from the primary investigators whenever possible. To avoid introducing bias, we obtained this unpublished information in writing, on forms designed for the purpose, and entered it into RevMan.

\section{Assessment of risk of bias in included studies}

Three review authors (SA, KC, C-FH) independently assessed risk of bias for each new study using the criteria outlined in Section 8.1 of the Cochrane Handbook for Systematic Reviews of Interventions (Higgins 2019). We resolved any disagreements by discussion or by involving another review author (MH). Although there are a number of scales devised for assessing the quality of RCTs, there is no convincing evidence that complex and time-consuming scales are more effective than simple scales (Verhagen 2001). We assessed the risk of bias according to the following domains.

- Random sequence generation.

- Allocation concealment: If allocation was performed using opaque envelopes we also categorised this as 'high risk' as it is not tamper-proof.

- Blinding of participants and personnel: For psychological interventions, we recognise that participants are unlikely to remain blinded however, we also categorised this as 'high risk'.

- Blinding of outcome assessment.

- Incomplete outcome data

- Selective outcome reporting: when a published trial protocol was not available we categorised this as unclear risk.

- Other bias.

We also provided a quote from the study to justify our judgment in the 'Risk of bias in included studies' table. When considering treatment effects, we have taken into account the risk of bias for the studies that contributed to that outcome.

\section{Measures of treatment effect}

\section{Dichotomous dato}

For all dichotomous outcomes, we calculated risk ratios (RRs) with 95\% confidence intervals (Cls) where appropriate, using randomeffects analyses.

\section{Continuous data}

For continuous data, if ordinal scale data appeared to be normally distributed, or if the analysis suggested parametric tests were appropriate, we treated the outcome measures as continuous. If there were at least two trials that reported the same outcomes, then we calculated a mean difference (MD) with $95 \%$ Cls across the trials. Where different outcome measures were used, we calculated a standardised mean difference (SMD) with 95\% Cls.

\section{Unit of analysis issues}

We predicted that randomisation would occur at the level of the individual participant in most, if not all, trials. Outcomes are reported at the end of treatment, and the end of follow-up, where data are available. Where trials included two or more active intervention arms and only one control arm (placebo, attention control, or usual care), we compared data from each treatment arm with data from the total number of participants in the control group, divided by the number of active intervention groups. The comparisons are presented as separate trials.

\section{Dealing with missing data}

We wrote to the authors of all included, ongoing, and dropout trials requesting data that were unavailable or ambiguous in the published articles. We received responses with additional data from the study authors of four new trials (Hoffman 2015; Kerr 2018; Robinson 2008; Wichowicz 2017). In 2008, we received responses with additional data from authors of two trials (Almeida 2006; Watkins 2007). In 2004 we received responses about six trials (Downes 1995; Forster 1996; Friedland 1992; House 2000; Reding 1986; Robinson 2000a; Sitzer 2002). We received a response from one further author, but no additional data had been provided by the time of publication (Rasmussen 2003). We received no responses from the remaining authors. We also wrote to all pharmaceutical companies known to produce, or have a licence to produce, pharmacological interventions in 2004. We received nine replies identifying no new trials, so we did not repeat this in the 2008 or current update.

\section{Assessment of heterogeneity}

We assessed clinical and methodological heterogeneity by examining the study characteristics. We used the 12 statistic to measure heterogeneity among the trials in each analysis (Deeks 2011). If there were at least two trials that reported the same outcomes, we reviewed the data for appropriateness of pooling. We interpreted the amount of heterogeneity as low $(0 \%$ to $29 \%$ ), moderate (30\% to $49 \%$ ), substantial (50\% to $89 \%)$, and considerable $(90 \%$ to $100 \%) I^{2}$ values. We reported similarities between interventions, participants, design, and outcomes in the included trials subsection. 


\section{Assessment of reporting biases}

We assessed publication bias by a funnel plot only if there were 10 or more included trials (Higgins 2019), using depression as the outcome of interest. We attempted to avoid language bias by including trials irrespective of language of publication, and translation was performed where needed by native speakers of that language. Review author (C-FH) translated and extracted data for the Chinese language papers. In some cases, similarities between trial reports indicated the possibility of multiple publications from the same trial. We contacted the authors to check whether the publications were duplicates. In the absence of a response and explicit cross-referencing, we judged articles to be from the same trial if they met the following criteria: 1) there was evidence of overlapping recruitment sites, study dates, and grant funding numbers, and 2) there were similar or identical reported participant characteristics in the trials.

\section{Data synthesis}

We analysed data using Review Manager software and pooled data for meta-analysis when trials assessed similar treatments and had similar outcomes (Review Manager 2014). We conducted a meta-analysis using available calculated mean difference (MD) or standardised mean differences (SMD) for continuous outcomes, and risk ratios (RRs) for dichotomous outcomes using a randomeffects analyses. In the results we included measures of uncertainty, such as $95 \%$ confidence intervals $(\mathrm{Cls})$ and estimates of $\mathrm{I} 2$. For all summary statistics in this review, please refer to the effects at the end of intervention, or at the end of follow-up.

\section{Summary of findings and certainty of the evidence}

We also assessed the certainty of evidence according to GRADE (Atkins 2004), by constructing a 'Summary of findings' table for the main outcomes per comparison using the GRADEPro tool (GRADEproGDT 2015; Schünemann 2019). We reported the relevant outcomes in the 'Summary of findings' table for each comparison.

These data were available for comparison: (1) pharmacological interventions versus placebo; and (2) one of various forms of psychological therapy versus usual care and/or attention control.

For the comparison 'Pharmacological intervention versus placebo', we reported the certainty of evidence for the following outcomes: meeting the study criteria for depression, scoring above cut-off points for a depressive disorder, mean depression scores, mean cognition scores, mean activities of daily living scores, and death at the end of treatment.

For the comparison 'Psychological interventions versus usual care and/or attention control', we reported the certainty of evidence for the following outcomes: meeting the study criteria for depression, scoring above cut-off points for a depressive disorder, mean depression scores, mean psychological distress scores, mean general health scores, mean social activities score, and death at the end of treatment.

We had no data for the remaining comparisons: (3) noninvasive brain stimulation and sham stimulation or usual care; (4) pharmacological intervention and one of various forms of psychological therapy versus pharmacological intervention plus usual care and/or attention control; (5) pharmacological intervention and one of various forms of psychological therapy versus placebo plus psychological therapy; (6) pharmacological intervention and non-invasive brain stimulation versus pharmacological intervention plus sham stimulation or usual care; (7) pharmacological intervention and noninvasive brain stimulation versus placebo plus non-invasive brain stimulation; (8) non-invasive brain stimulation and one of various forms of psychological therapy versus non-invasive brain stimulation plus usual care and/or attention control; and (9) non-invasive brain stimulation and one of various forms of psychological therapy versus sham brain stimulation or usual care plus psychological therapy. See Types of interventions.

\section{Subgroup analysis and investigation of heterogeneity}

We planned to undertake subgroup analyses to explore the influence of date of publication, sample size, duration of follow-up, treatment type, high (over 20\%) number of dropouts, and blinded versus unblinded outcome assessors. We performed subgroup analyses by method of assessment and outcome measures used to assess depression, psychological distress, general health, cognition, social activities, activities of daily living (ADL), disability, and anxiety for all comparisons.

If there were at least two trials that reported the same outcomes, we reviewed the data for appropriateness of pooling. If there was definite evidence of heterogeneity $(12>50 \%)$, we explored the potential reasons for the differences by performing subgroup analyses and meta-regression (Normand 1999). If the heterogeneity could not be explained, we combined the trials using randomeffects analyses with cautious interpretation, or did not combine them at all. Where possible, we performed subgroup analyses to examine the impact of treatment type and duration, and of stroke severity.

\section{Sensitivity analysis}

We planned to explore the sensitivity of the combined estimate of individual trials by leaving out one study if there was high risk of bias and methodological differences. We then calculated the combined effect of the remaining trials, and compared the results with the combined effect based on all the trials.

\section{RES U LTS}

\section{Description of studies}

\section{Results of the search}

In total, we identified 23,228 records, of these we retrieved 22,297 through database searching. We found 931 additional references by searching other resources. After duplicates were removed, we screened 9100 titles and abstracts and excluded 9069 irrelevant records. We retrieved full-text reports for the remaining 31 studies. After reading the full-texts, we excluded 17 studies as they did not meet the review eligibility criteria. We have provided the primary reasons for exclusions in the Characteristics of excluded studies table and Figure 1. We identified two trials that met the inclusion criteria (Hadidi 2014; Kim 2017). However, data were not available for non-depressed participants only. These trials are considered 'dropouts' (Table 1).

In the previous published version of this review (Hackett 2008), eight trials (Bramanti 1989; Downes 1995; Friedland 1992; Graffingo 2003; Leathley 2003; McCafferty 2000; Ohtomo 1985; Raffaele 1996) met the inclusion criteria but were considered 'dropouts' as data 
were not available for non-depressed participants only (Downes 1995; Raffaele 1996), and were either not available or not in a suitable format for meta-analysis (Bramanti 1989; Friedland 1992; Graffingo 2003; Leathley 2003; McCafferty 2000; Ohtomo 1985). See Table 1 for a more detailed information on these trials.

We received responses with additional data from authors of three new trials (Kerr 2018; Hoffman 2015; Wichowicz 2017).

\section{Included studies}

From the first published version of this review, there were a total of 12 included trials (14 interventions) (Creytens 1980; Dam 1996a/Dam 1996b; Forster 1996; Goldberg 1997; Grade 1998; House 2000; Palomaki 1999; Raffaele 1996; Rasmussen 2003; Reding 1986; Robinson 2000a/Robinson 2000b; Roh 1996). We included two trials in the 2008 update (Almeida 2006; Watkins 2007), resulting in 14 included trials (16 interventions), with 1515 participants at entry. Two trials compared two active treatments with placebo (Dam 1996a; Robinson 2000a). We compared data from both treatment arms in these trials with data from half the number of participants in the control groups, and presented the results as two separate studies (Dam 1996a/Dam 1996b; Robinson 2000a/Robinson 2000b). One trial included an attention control (where the time participants in the treatment group spent with a trained therapist was controlled for by attention control participants spending equal time with an untrained volunteer) as well as a control (usual care) group (House 2000). We combined data from the attention control and control group and compared these with data from the treatment group. One trial (Raffaele 1996) included in the first published version of the review (2004) is considered a 'dropout' following closer inspection of the results as data was not available for non-depressed participants only. More detailed information is provided in the Characteristics of included studies table.

In this update, we included six new trials with 417 participants (Hoffman 2015; Kerr 2018; Robinson 2008; Tsai 2011; Wichowicz 2017; Xu 2006). In total, 19 trials (21 interventions) involving 1771 participants are included.

\section{Participants}

All trials in this review included men and women. The average age of participants ranged from 55 to 73 years. Most of the trials reported the time between stroke and randomisation into the trial, with the range covering 'within three days' to six months. Most of the trials included participants with ischaemic stroke, diagnosed using a combination of standard clinical and computed tomography (CT) criteria. For more detailed information on each included study, please refer to the Characteristics of included studies table.

\section{Interventions}

Twelve trials (14 interventions) assessed pharmacological interventions compared to placebo (Almeida 2006; Creytens 1980; Dam 1996a/Dam 1996b; Grade 1998; Palomaki 1999; Rasmussen 2003; Reding 1986; Robinson 2000a/Robinson 2000b; Robinson 2008; Roh 1996; Tsai 2011; Xu 2006), and seven assessed psychological interventions compared to usual care and/or attention control (Forster 1996; Goldberg 1997; Hoffman 2015; House 2000; Kerr 2018; Watkins 2007; Wichowicz 2017). Results from these trials are presented and discussed separately.
There were no trials reporting on the remaining comparisons: (3) non-invasive brain stimulation and sham stimulation or usual care; (4) pharmacological intervention and one of various forms of psychological therapy versus pharmacological intervention plus usual care and/or attention control; (5) pharmacological intervention and one of various forms of psychological therapy versus placebo plus psychological therapy; (6) pharmacological intervention and non-invasive brain stimulation versus pharmacological intervention plus sham stimulation or usual care; (7) pharmacological intervention and non-invasive brain stimulation versus placebo plus non-invasive brain stimulation; (8) non-invasive brain stimulation and one of various forms of psychological therapy versus non-invasive brain stimulation plus usual care and/or attention control; and (9) non-invasive brain stimulation and one of various forms of psychological therapy versus sham brain stimulation or usual care plus psychological therapy. (See Types of interventions).

\section{Pharmacological interventions}

Among trials of pharmacological interventions, six compared a serotonin reuptake inhibitor (SSRI) against placebo (fluoxetine: Dam 1996a; Robinson 2000a; sertraline: Almeida 2006; Rasmussen 2003; escitalopram: Robinson 2008), and paroxetine: Xu 2006), one compared a serotonin antagonist and reuptake inhibitor against placebo (trazodone: Reding 1986), one compared a tricyclic antidepressant against placebo (nortriptyline: Robinson 2000b), and one compared a serotonin-norepinephrine reuptake inhibitor against placebo (milnacipran: Tsai 2011). Other treatments with antidepressant effects were used in five trials (piracetam: Creytens 1980; maprotiline: Dam 1996b; mianserin: Palomaki 1999; indeloxazine: Roh 1996), and a psychostimulant (methylphenidate: Grade 1998). Four trials (five interventions) used a fixed dosing regimen (Almeida 2006; Dam 1996a/Dam 1996b; Roh 1996; Xu 2006), and eight (nine interventions) used a flexible (Grade 1998; Rasmussen 2003; Robinson 2008), or escalating (Creytens 1980; Palomaki 1999; Reding 1986; Robinson 2000a/Robinson 2000b; Tsai 2011), regimen. Treatment duration varied from four weeks (Grade 1998; Reding 1986), to 52 weeks (Palomaki 1999; Rasmussen 2003; Robinson 2008; Tsai 2011).

\section{Psychological therapy}

Varying forms of psychological therapy were used in the included trials. Two trials stated explicitly that the intervention was problem-solving therapy (Forster 1996; House 2000), or cognitive behavioural coping therapy (Hoffman 2015). Two trials provided an intervention that was more broadly defined (home-based therapy: Goldberg 1997; and solution-focused brief therapy: Wichowicz 2017), and two provided motivational interviewing (Kerr 2018; Watkins 2007). Five trials used 'usual care' as the control comparison (Forster 1996; Goldberg 1997; Hoffman 2015; Kerr 2018; Watkins 2007), and one used usual care and attention control groups (House 2000). One trial stated that participants in the control comparison did not receive any psychotherapeutic interventions. The interventions were delivered by a variety of trained professionals, including specialist nurses (Forster 1996; Hoffman 2015; House 2000; Kerr 2018; Watkins 2007; Wichowicz 2017), and a mixed team of therapists (Goldberg 1997; Watkins 2007). Treatment duration varied from one visit per week for four weeks (Watkins 2007), to monthly home visits over one year (Goldberg 1997). 


\section{Outcomes}

\section{Primary outcome: depression}

Thirteen assessment scales were used to diagnose the proportion of people meeting the criteria for depression or scoring above the cut-off points for a depressive disorder in the 19 trials (21 interventions). The most commonly used measures were the Hamilton Depression Rating Scale (Almeida 2006; Dam 1996a; Dam 1996b; Grade 1998; Palomaki 1999; Rasmussen 2003; Robinson 2000a; Robinson 2000b; Robinson 2008; Tsai 2011), and the Hospital Anxiety Depression Scale (Hoffman 2015; Kerr 2018; Wichowicz 2017). Seven trials used two or more scales to assess abnormal mood or depression (Grade 1998; Hoffman 2015; House 2000; Kerr 2018; Palomaki 1999; Rasmussen 2003; Watkins 2007), and two older trials relied on a clinical or physician assessment (Reding 1986; Roh 1996).

\section{Secondary outcomes}

A variety of additional outcomes were assessed in each trial. Several studies assessed and reported outcome data for depression (Almeida 2006; Dam 1996a; Dam 1996b; Grade 1998; Hoffman 2015; House 2000; Kerr 2018; Palomaki 1999; Rasmussen 2003; Robinson 2000a/Robinson 2000b; Robinson 2008; Roh 1996; Tsai 2011; Watkins 2007; Wichowicz 2017; Xu 2006), psychological distress (House 2000; Watkins 2007), general health (Forster 1996), cognition (Almeida 2006; Grade 1998; Robinson 2000a/Robinson 2000b), social activities (Forster 1996; House 2000), activities of daily living Dam 1996a/Dam 1996b; Forster 1996; Hoffman 2015; House 2000; Reding 1986; Watkins 2007; Xu 2006), disability (Almeida 2006; Dam 1996a/Dam 1996b; Grade 1998; Robinson 2000a/Robinson 2000b), and anxiety (Hoffman 2015; Kerr 2018; Wichowicz 2017). A wide variety of additional measures were used in the trials (see Characteristics of included studies). Although most trials reported data from all the scales and assessments that were stated as used, these data were often not presented in a format that was easily collated for this review. We sought additional data from study authors if possible. Adverse events were reported in many studies (Almeida 2006; Creytens 1980; Dam 1996a/Dam 1996b; Grade 1998; House 2000; Palomaki 1999; Robinson 2000a/Robinson 2000b; Robinson 2008; Roh 1996; Tsai 2011; Watkins 2007); however, it was difficult to determine whether recording and reporting of adverse events were systematic. In other studies, adverse events were not reported by randomised group (Reding 1986), or only in a selected manner (Forster 1996; Goldberg 1997; Rasmussen 2003).

\section{Ongoing studies}

Two trials are ongoing (Sitzer 2002: pharmacological intervention; Kirkevold 2018: psychological therapy).

\section{Studies awaiting classification}

From the previous published version of this review, there were two trials listed as awaiting classification (Evans 1985; Katz 1998).
We were unable to obtain more information or outcome data from these trials, despite multiple attempts to contact the study authors. In the present review, there are four additional trials listed as awaiting classification (Chang 2011; IRCT201112228490N1; Ostwald 2014; Razazian 2016). We were unsure if depression was the primary outcome in two trials (IRCT201112228490N1; Razazian 2016). In the other two trials, no information regarding supervision was provided for the psychological therapy component of the intervention to help us determine if they meet our review criteria (Chang 2011; Ostwald 2014).

\section{Dropout studies}

In the previous published version of this review (Hackett 2008), seven trials met the inclusion criteria but were considered 'dropouts' as data were not available for non-depressed participants only (Downes 1995); or were either not available or not in a suitable format for meta-analysis (Bramanti 1989; Friedland 1992; Graffingo 2003; Leathley 2003; McCafferty 2000; Ohtomo 1985). See Table 1 for more detailed information on these trials.

We identified two new trials that met the inclusion criteria; however, data were not available for non-depressed participants only (Hadidi 2014; Kim 2017; Ostwald 2014). These trials are considered 'dropouts' (Table 1). One trial (Raffaele 1996) previously included in the first published version of the review (2004) is now considered a 'dropout' following closer inspection of the results as data was not available for non-depressed participants only.

We contacted the study authors of the dropout studies to request data for non-depressed participants only. However, we did not receive any responses.

\section{Excluded studies}

For this update, we excluded 17 trials at the full-text review stage. Reasons for exclusion include: 1 ) depression was not the primary outcome $(n=9) ; 2)$ unable to isolate data for stroke participants ( $n$ = 3); 3) data were not available for non-depressed participants at baseline $(n=2) ; 4)$ data were not available or in a suitable format for pooling ( $n=2)$ and; 5) participants were depressed at baseline $(n=1)$. We have listed the excluded studies, and their reasons for exclusion, in the Characteristics of excluded studies table.

\section{Risk of bias in included studies}

We present a graphical summary of 'Risk of bias' assessments performed by review authors for the included studies, based on the seven risk of bias domains (Figure 2). Figure 3 provides a summary of risk of bias for each included study. The reasons for judgements are provided in the 'Risk of bias' section in Characteristics of included studies. 
Figure 2. 'Risk of bias' graph: review authors' judgements about each risk of bias item presented as percentages across all included studies.

Random sequence generation (selection bias)

Allocation concealment (selection bias) Blinding of participants and personnel (performance bias): All outcomes Blinding of outcome assessment (detection bias): All outcomes Incomplete outcome data (attrition bias): All outcomes

Selective reporting (reporting bias)

Other bias
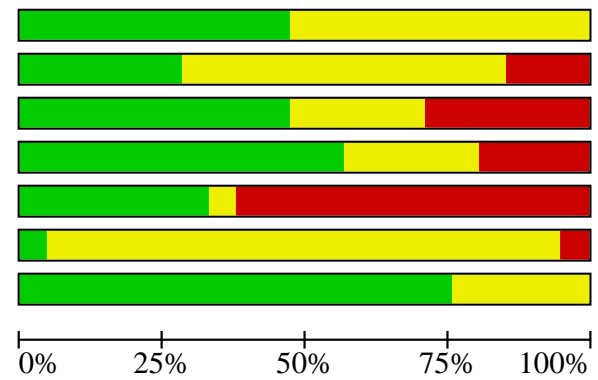
Figure 3. 'Risk of bias' summary: review authors' judgements about each risk of bias item for each included study.

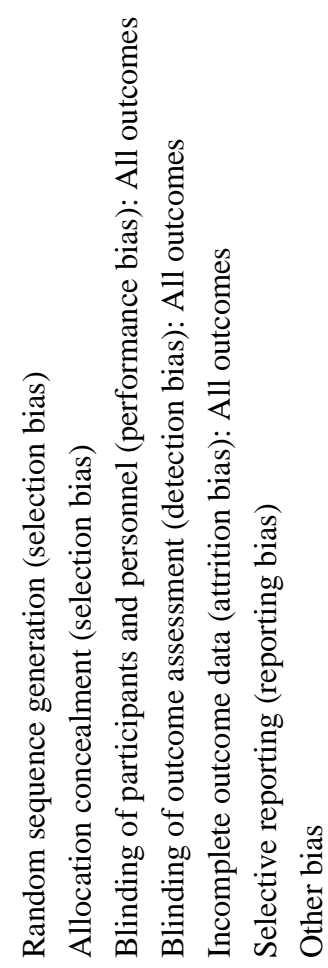

Almeida 2006

Creytens 1980

Dam 1996a

Dam 1996b

Forster 1996

Goldberg 1997

Grade 1998

Hoffman 2015

House 2000

Kerr 2018

Palomaki 1999

Rasmussen 2003

Reding 1986

Robinson 2000a

Robinson 2000b

Robinson 2008

Roh 1996

Tsai 2011

Watkins 2007

Wichowicz 2017

Xu 2006

\begin{tabular}{|c|c|c|c|c|c|c|}
\hline+ & + & + & ? & & $?$ & $?$ \\
\hline$?$ & $?$ & ? & ? & $\theta$ & ? & ? \\
\hline ? & \begin{tabular}{|l|}
$?$ \\
\end{tabular} & $?$ & + & & $?$ & + \\
\hline$?$ & $?$ & $?$ & + & & $?$ & + \\
\hline$?$ & ? & ? & $?$ & & $?$ & + \\
\hline+ & ? & $\theta$ & 0 & & $?$ & + \\
\hline+ & + & + & + & + & $?$ & + \\
\hline+ & $?$ & - & + & + & $?$ & + \\
\hline+ & + & e & 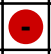 & + & $?$ & + \\
\hline+ & & - & $\odot$ & $\odot$ & $?$ & + \\
\hline$?$ & 0 & + & ? & $\odot$ & $?$ & $?$ \\
\hline$?$ & ? & + & + & & $?$ & $?$ \\
\hline+ & ? & + & + & & $?$ & + \\
\hline$?$ & $?$ & + & + & & $?$ & + \\
\hline$?$ & $?$ & + & + & & $?$ & + \\
\hline+ & + & + & + & + & $\theta$ & + \\
\hline+ & + & + & + & 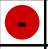 & $?$ & + \\
\hline$?$ & + & + & + & + & + & $?$ \\
\hline$?$ & $\theta$ & $\theta$ & $\theta$ & + & $?$ & + \\
\hline+ & $?$ & $\odot$ & + & + & $?$ & + \\
\hline$?$ & $?$ & $?$ & $?$ & $?$ & $?$ & + \\
\hline
\end{tabular}




\section{Allocation}

The randomisation sequence was appropriately generated in 10 trials (Almeida 2006; Goldberg 1997; Grade 1998; Hoffman 2015; House 2000; Kerr 2018; Reding 1986; Robinson 2008; Roh 1996; Wichowicz 2017), thus we rated these as low risk. However, nine trials (11 interventions) did not describe their method of sequence generation; we rated these as unclear risk (Creytens 1980; Dam 1996a/Dam 1996b; Forster 1996; Palomaki 1999; Rasmussen 2003; Robinson 2000a/Robinson 2000b; Tsai 2011; Watkins 2007; Xu 2006).

We rated six trials as low risk as an appropriately generated and clearly concealed allocation procedure was used (Almeida 2006; Grade 1998; House 2000; Robinson 2008; Roh 1996; Tsai 2011). Twelve trials (10 interventions) did not describe their method of allocation concealment and so we rated them as unclear risk (Creytens 1980; Dam 1996a/Dam 1996b; Forster 1996; Goldberg 1997; Hoffman 2015; Rasmussen 2003; Reding 1986; Robinson 2000a/Robinson 2000b; Wichowicz 2017; Xu 2006). One trial used opaque sealed envelopes (Watkins 2007), while another trial used coloured paper to conceal allocation (Kerr 2018), and one trial used sealed envelopes as their method of allocation concealment (Palomaki 1999), so they were all rated as high risk.

\section{Blinding}

Nine trials (10 interventions) reported that participants and personnel were blinded to the treatment allocation and so we rated these as low risk (Almeida 2006; Grade 1998; Palomaki 1999; Rasmussen 2003; Reding 1986; Robinson 2000a/Robinson 2000b; Robinson 2008; Roh 1996; Tsai 2011). Four trials (five interventions) did not provide information about blinding of participants and personnel so we rated them as unclear risk (Creytens 1980; Dam 1996a/Dam 1996b; Forster 1996; Xu 2006). We rated six trials as high risk (Goldberg 1997; Hoffman 2015; House 2000; Kerr 2018; Watkins 2007; Wichowicz 2017): due to the nature of the intervention in these trials, it is very unlikely that the participants and personnel remained blinded to the treatment allocation.

We rated nine trials (11 interventions) as low risk as outcome assessors were blinded to the treatment allocation (Dam 1996a/Dam 1996b; Grade 1998; Hoffman 2015; Rasmussen 2003; Robinson 2000a/Robinson 2000b; Robinson 2008; Roh 1996; Tsai 2011; Wichowicz 2017). Five trials did not provide information about blinding of outcome assessors and so we rated them as unclear risk (Almeida 2006; Creytens 1980; Forster 1996; Palomaki 1999; Xu 2006). Three trials had blinded outcome assessors, however it is likely that they became aware of the treatment allocation and we therefore rated them as high risk (Goldberg 1997; House 2000; Kerr 2018). In one trial, participants were sent questionnaires to complete on their own and so we also rated this as high risk (Watkins 2007).

\section{Incomplete outcome data}

We rated seven trials as low risk as they provided intentionti-treat (ITT) analyses (Grade 1998; Hoffman 2015; House 2000; Robinson 2008; Tsai 2011; Watkins 2007; Wichowicz 2017). One trial reported completing ITT analyses but not how they handled missing data so we rated it as unclear risk (Xu 2006). We rated 11 trials (13 interventions) as high risk for attrition bias as they reported per-protocol analyses only (Almeida 2006; Creytens 1980; Dam 1996a/Dam 1996b; Forster 1996; Goldberg 1997; Kerr
2018; Palomaki 1999; Rasmussen 2003; Reding 1986; Robinson 2000a/Robinson 2000b; Roh 1996).

\section{Selective reporting}

We rated one trial as low risk as all pre-specified outcomes from the trial protocol were reported in the publication (Tsai 2011). We rated 17 trials (19 interventions) as unclear risk as all pre-specified outcomes were reported but no trial protocol was available to compare with the publication (Almeida 2006; Creytens 1980; Dam 1996a/Dam 1996b; Forster 1996; Goldberg 1997; Grade 1998; Hoffman 2015; House 2000; Kerr 2018; Palomaki 1999; Rasmussen 2003; Reding 1986; Robinson 2000a/Robinson 2000b; Roh 1996; Watkins 2007; Wichowicz 2017; Xu 2006). We rated one trial as high risk as one outcome reported in the study protocol or trial registry information page was not reported in the primary results publication (Robinson 2008).

\section{Other potential sources of bias}

We rated 14 trials (16 interventions) as low risk as the baseline demographic characteristics were balanced between the groups (Dam 1996a/Dam 1996b; Forster 1996; Goldberg 1997; Grade 1998; Hoffman 2015; House 2000; Kerr 2018; Reding 1986; Robinson 2000a/Robinson 2000b; Robinson 2008; Roh 1996; Watkins 2007; Wichowicz 2017; Xu 2006). We rated five trials as unclear risk as either no information about baseline demographics between groups were provided or we were unclear how differences in age or the proportion of participants with heart disease in one group may pose bias (Almeida 2006; Creytens 1980; Palomaki 1999; Rasmussen 2003; Tsai 2011).

\section{Effects of interventions}

See: Summary of findings 1 Pharmacological interventions (antidepressants) compared to placebo for preventing depression after stroke; Summary of findings 2 Psychological therapy compared to usual care and/or attention control for preventing depression after stroke

See 'Summary of findings 1; Summary of findings 2'.

Overall, we included 1930 participants in this review. In view of the large number and heterogeneous nature of the outcome measures and the reporting of results, we considered it inappropriate to pool outcome data for many endpoints. For details of the comparisons made for the studies with outcome data, refer to the Data and analyses section (as outlined in the protocol, data for trials of psychostimulants (Grade 1998) are presented separately).

\section{Primary outcome}

\section{Depression}

\section{Meeting the study criteria for depression at the end of treatment}

Eight trials, nine pharmacological interventions, found favourable treatment effects (risk ratio (RR) $0.50,95 \%$ confidence interval (CI) 0.37 to $0.68,734$ participants, very low-certainty evidence, Analysis 1.1) for this outcome at the end of treatment compared to placebo (Almeida 2006; Palomaki 1999; Rasmussen 2003; Robinson 2000a/Robinson 2000b; Robinson 2008; Roh 1996; Tsai 2011; Xu 2006). No heterogeneity was observed but confidence intervals were very wide. 
Two trials of psychological therapy revealed favourable treatment effects (RR $0.68,95 \% \mathrm{Cl} 0.49$ to $0.94,607$ participants, very lowcertainty evidence, Analysis 2.1) for this outcome at the end of treatment compared to usual care and/or attention control (House 2000; Watkins 2007). No heterogeneity was observed but confidence intervals were very wide.

\section{Other outcomes}

\section{Depression}

\section{Average change in scores between baseline and end of treatment}

Three trials, five pharmacological interventions, found no statistically significant difference in the average change in depression scores between baseline and the end of treatment between those who received pharmacological interventions compared to placebo (standardised mean difference (SMD) -0.05 , $95 \% \mathrm{Cl}-0.33$ to 0.23 , 211 participants, Analysis 1.3) (Almeida 2006; Dam 1996a/Dam 1996b; Robinson 2000a/Robinson 2000b). No heterogeneity was observed but confidence intervals were very wide.

We did not perform a meta-analysis for the comparison between psychostimulants and placebo (Analysis 3.1), as only one trial contributed data to this outcome (Grade 1998).

\section{Mean scores at the end of treatment}

Two trials, four pharmacological interventions, revealed no statistically significant difference in mean depression scores at the end of treatment between the pharmacological intervention and placebo groups (MD 0.59, 95\% Cl -1.46 to $2.63,100$ participants, very low-certainty evidence, Analysis 1.4) (Dam 1996a/Dam 1996b; Robinson 2000a/Robinson 2000b). No heterogeneity was observed but confidence intervals were very wide.

We did not perform a meta-analysis on this outcome for the comparison psychological therapy versus usual care and/or attention control (Analysis 2.3), due to the heterogenous nature of the outcome measures.

\section{Mean scores at the end of follow-up}

We did not perform a meta-analysis on this outcome for the comparison: psychological therapy versus usual care and/or attention control (Analysis 2.4), due to the heterogenous nature of the outcome measures.

\section{Psychological distress}

Two trials of psychological therapy found favourable treatment effects in the average change in psychological distress scores between baseline and the end of treatment (MD -1.37, 95\% Cl -2.27 to $-0.48,607$ participants, Analysis 2.5) compared to usual care or attention control (House 2000; Watkins 2007). No heterogeneity was observed but confidence intervals were very wide.

We did not perform a meta-analysis on the mean psychological distress scores at the end of treatment for the comparison psychological therapy versus usual care and/or attention control (Analysis 2.6), due to the heterogenous nature of the outcome measures.

\section{General health}

We did not perform a meta-analysis on the average change and mean general health scores at the end of treatment for the comparison psychological therapy versus usual care and/ or attention control (Analysis 2.7; Analysis 2.8), due to the heterogenous nature of the outcome measures.

\section{Cognition}

\section{Average change in scores between baseline and end of treatment}

Two trials, three pharmacological interventions, found no statistically significant difference between the pharmacological intervention and placebo groups at the end of treatment (MD 0.11, $95 \% \mathrm{Cl}-1.02$ to $1.24,159$ participants, very low-certainty evidence Analysis 1.5) (Almeida 2006; Robinson 2000a/Robinson 2000b). No heterogeneity was observed but confidence intervals were very wide.

We did not perform a meta-analysis on this outcome for the comparison pharmacological interventions versus placebo (psychostimulants) as only one study contributed data to this outcome (Analysis 3.2).

\section{Mean scores at the end of treatment}

One trial, two pharmacological interventions, revealed no statistically significant difference between the pharmacological intervention and placebo groups at the end of treatment (MD $-0.42,95 \% \mathrm{Cl}-2.60$ to $1.76,48$ participants, Analysis 1.6) (Robinson 2000 a/Robinson 2000b). No heterogeneity was observed but confidence intervals were very wide.

\section{Social activities}

Two trials of psychological therapy found no statistically significant difference between the psychological therapy and usual care and/ or attention control groups at the end of treatment (MD - $0.39,95 \%$ $\mathrm{Cl}-3.81$ to 3.03, 690 participants, Analysis 2.10) (Forster 1996; House 2000). There was $80 \%$ heterogeneity and very wide confidence intervals.

\section{Activities of daily living}

\section{Average change in scores between baseline and end of treatment}

Two trials, three pharmacological interventions, found no statistically significant difference between the pharmacological intervention and placebo groups at the end of treatment (MD 1.18, $95 \% \mathrm{Cl}-7.77$ to $10.14,57$ participants, very low-certainty evidence, Analysis 1.7) (Dam 1996a/Dam 1996b; Reding 1986).

Three trials of psychological therapy revealed no statistically significant difference between the psychological therapy and usual care and/or attention control groups at the end of treatment (MD $0.29,95 \% \mathrm{Cl}-0.18$ to $0.77,847$ participants, very low-certainty evidence, Analysis 2.11) (Forster 1996; House 2000; Watkins 2007).

\section{Mean scores at the end of treatment/follow-up}

Two trials, three pharmacological interventions, found no favourable treatment effects compared to placebo (MD -3.86, 95\% $\mathrm{Cl}-9.48$ to $1.77,116$ participants, Analysis 1.8) (Dam 1996a/Dam 1996b; Xu 2006). There was minimal heterogeneity $\left(I^{2}=22 \%\right)$ and very wide confidence intervals were observed. 
We did not perform a meta-analysis on this outcome for the comparison psychological therapy versus usual care and/ or attention control (Analysis 2.12; Analysis 2.13), due to the heterogenous nature of the outcome measures.

\section{Disability}

We did not perform a meta-analysis on this outcome for the comparison pharmacological interventions versus placebo (antidepressants) (Analysis 1.9; Analysis 1.10), due to the heterogenous nature of the outcome measures.

We did not perform a meta-analysis on this comparison of pharmacological interventions versus placebo (psychostimulants), as only one trial contributed data to this outcome (Analysis 3.3).

\section{Anxiety}

We did not perform a meta-analysis on this outcome for the comparison psychological therapy versus usual care and/ or attention control (Analysis 2.14; Analysis 2.15), due to the heterogenous nature of the outcome measures.

\section{Adverse events: death}

Eight trials, nine pharmacological interventions found no statistically significant difference in death between those who received pharmacological interventions compared to placebo (RR $1.25,95 \% \mathrm{Cl} 0.32$ to $4.91,496$ participants, very low-certainty evidence, Analysis 1.12) (Creytens 1980; Dam 1996a/Dam 1996b; Palomaki 1999; Robinson 2000a/Robinson 2000b; Robinson 2008; Roh 1996; Xu 2006). No heterogeneity was observed but confidence intervals were very wide.

Five trials of psychological therapy showed no statistically significant difference between the psychological therapy and usual care and/or attention control groups. No heterogeneity was observed but confidence intervals were very wide (RR 1.18, 95\% $\mathrm{Cl} 0.73$ to 1.91 , 975 participants, very low-certainty evidence, Analysis 2.16) (Forster 1996; House 2000; Kerr 2018; Watkins 2007; Wichowicz 2017).

\section{Adverse events: all}

There was no statistically significant harm in the pharmacological interventions versus placebo (antidepressants) or psychological therapy versus usual care and/or attention control comparisons. See Analysis 1.13; Analysis 2.17. One trial reported that psychostimulants resulted in no deaths at the end of treatment (Analysis 3.4).

\section{Adverse events: leaving the study early (including death)}

Ten trials, 12 pharmacological interventions, found no statistically significant difference between the pharmacological interventions and placebo groups at the end of treatment (RR 1.03, 95\% Cl 0.84 to 1.26, 836 participants, Analysis 1.14) (Almeida 2006; Creytens 1980; Dam 1996a/Dam 1996b; Palomaki 1999; Rasmussen 2003; Robinson 2000a/Robinson 2000b; Robinson 2008; Roh 1996; Tsai 2011; Xu 2006). No heterogeneity was observed but confidence intervals were very wide.

We did not perform a meta-analysis on this outcome for the comparison pharmacological interventions versus placebo (psychostimulants), as only one trial contributed data to this outcome (Analysis 3.5).
Six trials of psychological therapy revealed no statistically significant difference between the psychological therapy and usual care and/or attention control groups (RR $1.30,95 \% \mathrm{Cl} 0.90$ to 1.88, 1030 participants, Analysis 2.18) (Forster 1996; Goldberg 1997; House 2000; Kerr 2018; Watkins 2007; Wichowicz 2017). No heterogeneity was observed but confidence intervals were very wide.

\section{DISCUSSION}

\section{Summary of main results}

We added six new trials (417 participants) to this review update, resulting in 19 included trials (21 interventions, 1771 participants). Data were available for 12 pharmacological trials (14 interventions) and seven psychological trials. We found no trials of other included interventions, or their combination.

\section{Pharmacological therapy trials}

Compared with placebo, there is very low-certainty evidence which shows that pharmacological therapy reduced the incidence of depression after stroke. The very low-certainty rating was due to the variety of study methods and endpoints measured, inadequate reporting of allocation concealment, evidence of attrition bias (high numbers of dropouts in several of the studies), and wide confidence intervals. Because of this low certainty we have previously only provided a narrative review of data for pharmacological therapy and concluded that the evidence did not indicate that it was effective in preventing depression after stroke (Hackett 2008). In other non-stroke trials, where antidepressants did not result in a clinically significant reduction in depressive symptoms, benefits were indicated on other emotional symptoms, such as reducing anxiety and improving self-rated mental health and quality of life (e.g. Lewis 2019). There was no evidence of adverse effects or death associated with the use of pharmacological therapy to prevent depression after stroke. However, important known adverse effects such as falls and hip fracture apparent in other stroke trials (FOCUS 2019), were not reported, or the included trials were too small to identify them or, most likely, they were not systematically recorded.

The pharmacological therapy trials included participants whose time from onset of stroke to randomisation ranged from within three days to 13 weeks. Duration of the interventions ranged from three weeks to 52 weeks. This is important because of the serious debate about withdrawal effects and uncertainty about the balance of risk and benefit in longer-term prescribing. The prescription of antidepressants in this population is usually started in stroke services and maintained in primary care. This means important uncertainty remains for service providers and the evidence on which to guide pharmacological therapy is limited.

\section{Psychological therapy trials}

Compared to usual care or attention control, there is very low-certainty evidence which shows that psychological therapy reduced the incidence of depression after stroke at the end of treatment. No new trials reported on this outcome so these results are the same as in the previous review (Hackett 2008). As for pharmacological therapy, potential minor benefits on nonspecific symptoms are not covered. The certainty of evidence was very low due to inadequate reporting of allocation concealment and blinding of participants, personnel and outcome assessment, evidence of attrition bias (high numbers of dropouts in several of 
the studies), and wide confidence intervals. There was no evidence of adverse effects or death associated with psychological therapy to prevent depression after stroke.

Psychological therapy trials included participants whose time from onset of stroke to randomisation ranged from within days to 30 weeks. It is difficult to consider trials including participants six months after their stroke as true prevention trials. Duration of treatment was between four weeks and 52 weeks.

\section{Non-invasive brain stimulation or combinations of included intervention trials}

We found no trials of non-invasive brain stimulation compared with sham stimulation or usual care, or a combination of any of the included interventions with only one of the included interventions, to prevent depression after stroke.

\section{Other trials}

There were 10 trials we considered as 'dropouts' as outcome data were not analysed by depression status at baseline $(n=4)$ or not in a suitable format for pooling $(n=6)$. There are six trials awaiting classification. We were unable to obtain more information or outcome data in two trials. We were unsure if depression was the primary outcome in two trials, and in three trials we were unable to determine if the psychological therapy component meets the review criteria. Two ongoing trials (one psychological and one pharmacological therapy) will contribute more evidence for future updates of this review. The identification of ongoing trials, dropout trials, and trials awaiting classification indicate that this is an area of research for which further evidence will evolve in the short and longer term.

A major but not unexpected finding was the considerable heterogeneity in design, analysis, and reporting of clinical trials in this area of stroke medicine. To begin with, the trials included participants whose time from the onset of stroke to randomisation ranged from several days to seven months. It is likely that the aetiology of depression and response to treatment will vary across the different stages of recovery from stroke; for example, people who are in the acute phase of stroke are likely to be adjusting to experiencing a potentially life-threatening event as well as rapidly evolving effects of cerebral ischaemia. On the other hand, those who survive long term are adjusting to any fixed neurological deficits and residual disability, with varying impact on their social and financial situation. In a similar manner, the effects of treatment are likely to vary according to whether they are administered for short (several weeks) or long (several months to a year) time periods.

Another aspect of heterogeneity was the inclusion criteria across trials. Ideally, participants should be homogeneous with regard to stroke diagnosis, which requires the use of standard diagnostic criteria and neuroimaging in a high proportion of cases. Several of the pharmacological therapy trials, and only one of the psychological therapy trials, reported the method used to diagnose stroke, and while most did not provide details regarding stroke subtype, four trials included participants with subarachnoid haemorrhage. Given differences in the natural history, management, and also potential aetiological factors for depression in the setting of subarachnoid haemorrhage, it could be argued that this form of stroke should be examined separately to atherosclerotic stroke.
With regard to other entry criteria, many trials excluded people with communication problems, cognitive impairment, and previous psychiatric illness. This may be appropriate for trials of psychological therapy, as participants are required to engage actively in talking therapies. However, use of a large list of exclusions in trials of pharmacological therapy means that the results are likely to be applicable only to a small proportion of survivors of stroke who have a narrow range of co-morbidity and other characteristics. This reinforces a common criticism of depression research in the general population that the trial participants are not representative of those requiring treatment in the 'real world' (Zimmerman 2002). It would appear that this criticism is also applicable to trials to prevent depression after stroke, where up to half of survivors may be excluded using such criteria (Turner-Stokes 2003). Given the older age of most people with stroke, and the frequent presence of neurological impairments, aphasia, and co-morbid medical conditions, the fact that up to half of survivors are excluded from these trials limits the external validity of the results.

While it is relatively easy to diagnose major vascular disease endpoints in clinical trials, defining depression as an outcome can be problematic. Disturbances of language and cognition are common after stroke, and physical and other behavioural cues, such as slowness, reduced appetite, and loss of facial expression, may be misleading in the diagnosis of abnormal mood. Although gold-standard diagnostic definitions are available for major depression, dysthymia (Persistent Depressive Disorder) and other mood disorders (for example, APA 1987, APA 1994, APA 2013, and ICD 10 1992), these require modification to use among people with stroke. Clearly, there is the need to accept stroke as the concomitant medical condition, but also the presence of symptoms for less than the two years required for a diagnosis of dysthymia. In this situation minor depression (APA 1994), which is characterised by the presence of two to four depressive symptoms during a twoweek period, and requires one of these symptoms to be either depressed mood or loss of interest or pleasure, may be more appropriate.

In clinical practice, a trained health professional establishes the presence of abnormal mood by interviewing a person. Given the practical difficulties and high cost of conducting detailed psychiatric interviews on participants in clinical trials, we considered it appropriate to adopt a pragmatic approach and determine a case of depression on the basis of a psychiatric interview or use of a validated mood questionnaire. However, there are several problems associated with this approach. First, some studies included participants whose baseline mood scores were within the range for abnormal mood or depression. As we consider it inappropriate to undertake a study of the prevention of depression when it may already be present, we have elected to exclude these participants in these studies until data are available from the researchers. Second, the multiple approaches and scales used to assess mood across studies, and the variation in reporting of data within and between studies, made the pooling of data inappropriate in most cases. Finally, since primary a priori endpoints were seldom reported for the multiple measures used, it was difficult to assess whether the data presented were truly the intended primary outcomes in many studies, particularly given the high number of studies with selective reporting of outcomes. 
Another important aspect of trial design is the sample size required to detect a clinically meaningful effect; that is, how much of a difference between groups is considered reasonable to expect. While the continuous measures offered by various mood scales are appealing, as only 50 to a few hundred participants may be required in trials, the clinical significance of a few points difference between group means on follow-up is not so clear cut. Transformation of scores according to particular cut-off values, or the assessment of people using standardised clinical diagnostic criteria to provide a categorical disease endpoint is more meaningful, but may require several thousand participants. In addition, participants in clinical trials are likely to be more biologically robust and may potentially receive a treatment effect by virtue of their participation alone.

Finally, it is difficult to interpret the significance of outcomes when there is inadequate concealment of randomisation and high numbers of dropouts in several of the studies. Few trials systematically recorded and reported adverse events, making it impossible to undertake a reliable assessment of the benefits and risks of therapy. There was inconsistency in the methods of analysis and reporting of results, with most studies presenting per-protocol analyses. For trials with high dropouts (more than $20 \%$ ), intentionto-treat (ITT) analysis becomes very important. Should ITT (with missing data for the best possible and worst possible outcome) and per-protocol analyses indicate similar trends, then the findings are likely to be interpreted as clinically robust.

In summary, the lack of evidence of an effect of pharmacological and psychological therapy for preventing depression after stroke can be readily attributed to the limited numbers of studies, many of which are limited by poor study design, analysis, and reporting, as much as to the possible lack of limited efficacy of the interventions.

\section{Overall completeness and applicability of evidence}

The present review included 19 trials (21 interventions), with 1771 participants. Data were available for 12 pharmacological trials (14 interventions) and seven psychological therapy trials. We considered 11 trials as 'dropouts' as outcome data were not available in a suitable format for the review (six trials), or were not presented by depressed and not depressed participants at baseline (five trials). Five trials (two pharmacological interventions and three psychological therapies) are awaiting classification Two trials (pharmacological interventions and psychological therapy) are ongoing.

The accuracy of the findings of this systematic review and metaanalysis is based on the trials which met the eligibility criteria. These findings must be considered in light of several basic methodological limitations of many of these trials, including the short duration of many interventions, variation in the types of trial participants recruited, the methods used to diagnose depression, lack of an a priori measurable endpoint, and the generally poor design, outcome assessment, analysis and interpretation of results. We will incorporate new data in future updates.

\section{Quality of the evidence}

We rated the certainty of evidence for all comparisons using the five GRADE considerations (study limitations, consistency of effect, indirectness, imprecision and publication bias; Schünemann 2019). We created a 'Summary of findings' table for each comparison. Overall, we assessed the certainty as very low.

\section{Limitations in study design or execution}

For the comparison of pharmacological interventions with placebo, we downgraded the certainty of evidence for the following outcomes: meeting the study criteria for depression at end of treatment, mean depression scores, mean cognition scores, mean activities of daily living scores, and death at end of treatment by two points as we rated several trials as having a high or unclear risk of bias in multiple domains. See Summary of findings 1.

For the comparison of psychological interventions with usual care and/or attention control, we downgraded the certainty of evidence for the following outcomes: meeting the study criteria for depression at end of treatment, mean social activities scores, and death at end of treatment by two points as we rated several trials as having a high risk in multiple risk of bias domains. See Summary of findings 2 .

\section{Inconsistency of results}

For the comparisons of pharmacological interventions with placebo, we did not downgrade the certainty of evidence for inconsistency of results. No heterogeneity between the studies was observed. See Summary of findings 1;

For the comparison of psychological therapy with usual care and/ or attention control, we downgraded the social activities scores by two points for substantial heterogeneity $\left(1^{2}=80 \%\right)$. See Summary of findings 2.

\section{Indirectness of evidence}

All included trials addressed the main review questions (PICO): 1) the effect of pharmacological interventions compared to placebo; and 2) psychological therapy compared to usual care and/or attention control in preventing depression or depressive symptoms after stroke. Thus, we did not downgrade any outcomes in any comparisons for indirectness of evidence. See Summary of findings 1 ; Summary of findings 2 .

\section{Imprecision}

For the comparison of pharmacological interventions with placebo, we downgraded the certainty of evidence for meeting the study criteria for depression at the end of treatment by one point because the confidence intervals were wide. We also downgraded the certainty of evidence for the following outcomes: mean depression scores, mean cognition scores, mean activities of daily living scores, and death at end of treatment, because the confidence intervals were very wide. See Summary of findings 1.

For the comparison of psychological interventions with usual care and/or attention control, we downgraded the certainty of evidence for meeting the study criteria for depression at end of treatment by one point because the confidence intervals were wide. We also downgraded the certainty of evidence for the following outcomes: mean social activities score and death at end of treatment, because the confidence intervals were very wide. See Summary of findings 2.

\section{Publication bias}

We assessed publication bias using funnel plots for the outcome: meeting the study criteria for depression at end of treatment for the pharmacological interventions versus placebo comparison. We 
deemed this necessary to show no evidence of publication bias for this outcome (Figure 4), as there were nine trials (close to 10 trials, the usual minimum requirement) contributing to the analysis. We did not assess publication bias using funnel plots for the other outcomes due to the small number of trials ( $<10$ trials) contributing to the analysis. Therefore, we did not downgrade the certainty of evidence for publication bias in any of the outcomes. See Summary of findings 1; Summary of findings 2.

Figure 4. Funnel plot of comparison: 1 Pharmacological interventions (antidepressants) versus placebo, outcome: 1.1 Depression: meeting study criteria for depression at end treatment.

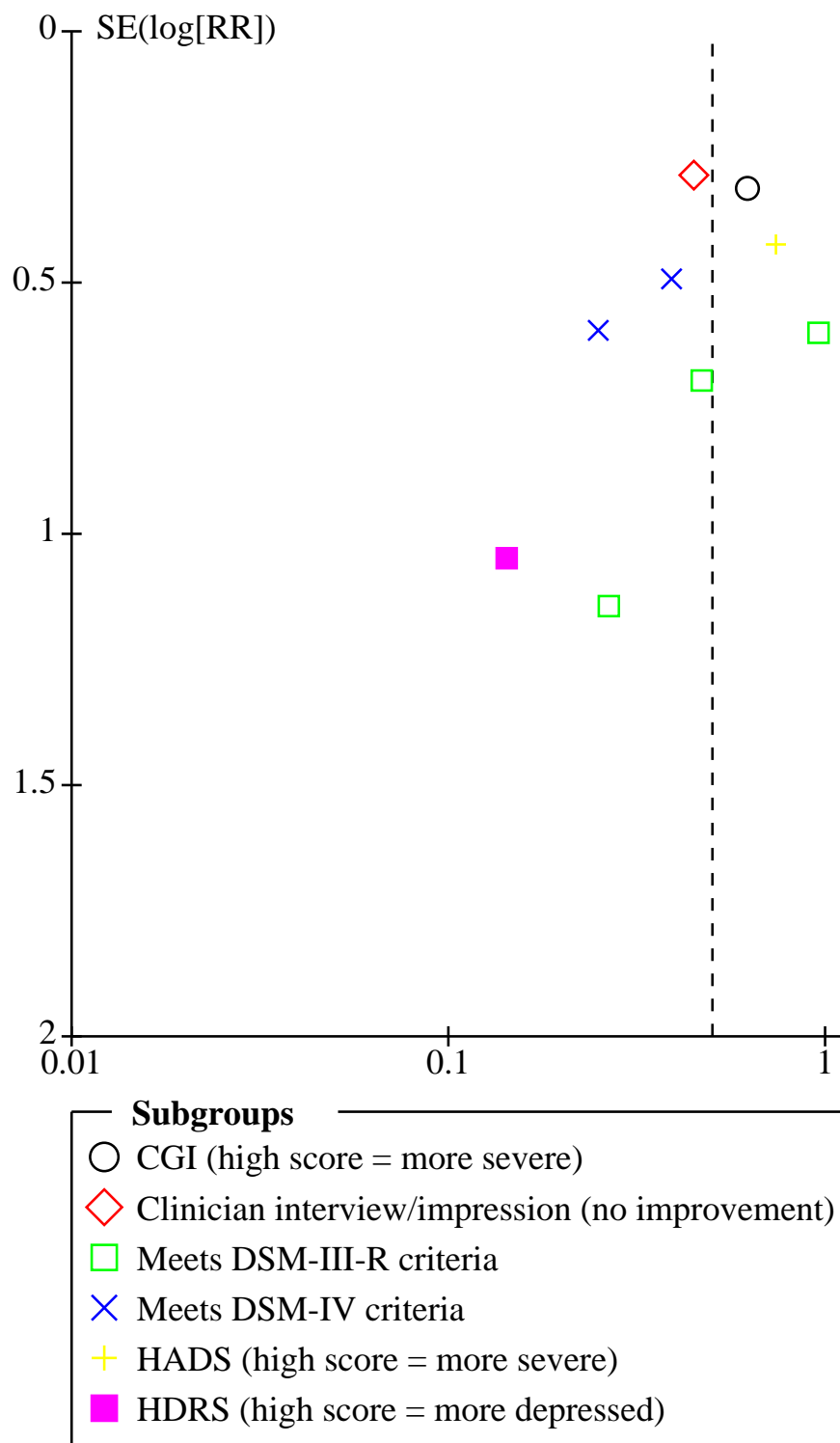

\section{Potential biases in the review process}

\section{Strengths and weaknesses of this review}

This review has rigorously adhered to the Cochrane methods for performing systematic reviews. During the review process we have tried to avoid and minimise any biases. We undertook extensive searches of databases and additional resources. We did not apply any language restrictions within the search process. Thus, we believe that we have identified and included all potentially relevant trials in this review. We arranged for any potentially relevant non-
English full-text trials to be translated into English to finalise the eligibility process. Furthermore, at least two review authors independently extracted and managed the data.

The main weakness of this review is the heterogenous nature of the outcome measures and the frequent use of multiple scales between and within trials. As a result, we were unable to pool outcome data for many endpoints. Furthermore, inadequate reporting for some of the trials has precluded classification of risk of bias as either low or high risk. This has led us to rate some of the trials across the 
categories as having an unclear risk of bias and an overall rating of 'very low' certainty of evidence.

\section{Agreements and disagreements with other studies or reviews}

To date, there are no other systematic reviews as comprehensive as the current review.

We found one other systematic review comparing the effect of pharmacotherapy to prevent depression in the stroke population (Salter 2013). This review is similar to our review, particularly in their inclusion of trials with people with no diagnosed depressive disorder or a mood score below the standard cut-off score for a depressive disorder at baseline. The authors included eight trials, of which six trials (seven interventions) (Almeida 2006; Palomaki 1999; Rasmussen 2003; Robinson 2000a/Robinson 2000b; Robinson 2008; Tsai 2011) are included in our review. In contrast to our review, that review combined the results from both active treatment conditions/arms (nortriptyline and fluoxetine) from the Robinson 2000a trial in their analysis. We excluded the other two included trials as one trial did not have a placebo comparison and the other trial had motor function as the primary outcome. The Salter 2013 review found a significant reduced risk for depression (odds ratio (OR $0.34,95 \% \mathrm{Cl} 0.22$ to 0.53 ) based on satisfactory methodological quality according to the Jadad scale (Clark 1999). Similarly, they also found that the reporting of adverse events was generally poor, making it difficult to undertake a reliable and meaningful evaluation of the relative risks for adverse events associated with prophylactic therapy.

We found a more targeted systematic review of fluoxetine to prevent depression in the stroke population (Yi 2010). Only two of their six included trials are included in our review (Dam 1996a; Robinson 2000a). We excluded the remaining four of their included trials as they did not have a placebo comparison. Yi 2010 demonstrated that fluoxetine reduced the incidence of depression after stroke (OR $=0.25,95 \% \mathrm{Cl} 0.11$ to 0.56$)$, with no evidence of adverse events (OR) $0.88,95 \% \mathrm{Cl} 0.31$ to $2.49, \mathrm{P}=0.82$ ). The authors noted several methodological limitations including inadequate randomisation sequence, randomisation concealment, and dropout rates ranging from $0 \%$ to $11.1 \%$ in the fluoxetine groups and from $0 \%$ to $14.3 \%$ in the control groups.

There were no systematic reviews comparing the effect of repetitive transcranial magnetic stimulation (rTMS) to sham rTMS, or of a combination of rTMS and pharmacotherapy to usual care or sham rTMS and pharmacotherapy to prevent depression after stroke. There is one systematic review evaluating the use of rTMS in people diagnosed with major depressive disorder in the general populations rather than a population with stroke (Rachid 2018). However, rTMS was not used to prevent depression but more as maintenance therapy to prevent relapse of depression.

One systematic review reported on the effect of psychotherapy for preventing depression after stroke (Baker 2018). The authors included five trials, of which one (Hoffman 2015), is included in our review. We excluded the remaining four trials as the intervention in two did not meet the review criteria, one trial only included caregivers, and the remaining trial did not have depression as the primary outcome. The review authors did not perform a metaanalysis and only described the included studies narratively .
The identification of studies currently ongoing and those awaiting classification indicate that this is an area of stroke research for which further evidence will evolve in the short and longer term.

\section{AUTHORS' CONCLUSIONS}

\section{Implications for practice}

There is inadequate evidence at present to support the routine use of antidepressants, psychostimulants, other drugs, or psychological therapies to prevent depression and improve recovery after stroke. All positive effects are based on very lowcertainty evidence. The small positive benefit of psychological strategies probably endorses the use of more structured approaches to the delivery of education and advice targeting emotional recovery and adjustment to the effects of stroke. However, the amount of evidence to support the routine use of these approaches in stroke rehabilitation is limited, as is the generalisability of these findings to all stroke survivors due to the narrow inclusion and exclusion criteria for participants in these trials.

\section{Implications for research}

One reason for uncertainty in this area is that previous trials have been of inadequate design. There is a need for further research using more rigorous methods. In addition to concealment of randomisation, and blinded treatment allocation and outcome assessments, future trials of pharmacological and psychological therapy for the prevention of depression after stroke should be designed with attention to the methodological issues described below.

- The trial should be of adequate power to detect modest but clinically important effects on a binary endpoint of significantly abnormal mood state, defined either by structured psychiatric interview or a validated mood scale.

- Participants should be recruited early (within four to six weeks) after the onset of stroke and the treatment continued for at least six months (the high-risk period), to allow the maximum effect on the natural history of the disorder and evaluation of a treatment regimen that could be relatively easily adopted into clinical practice.

- Standard clinical criteria for stroke should be used, and people with subarachnoid haemorrhage and those depressed at baseline excluded from participation.

- Exclusion criteria should be kept to a minimum without forgoing safety to allow trial results to be generalisable to most stroke survivors.

- A priori primary and secondary outcomes should be stated.

- Careful prospective assessment and complete reporting of adverse events should be undertaken.

- A limited number of key outcomes should be measured and reported.

\section{ACKNOWLEDGEMENTS}

The 2004 published version of this review was supported by a grant from the Stroke Society of Australasia in 2003, with additional financial assistance provided by the Academic Unit of Psychiatry, the University of Leeds, and the Department of Clinical Neurosciences, the University of Edinburgh. We would 
like to thank the Cochrane Stroke Group, particularly Brenda Thomas and Josh Cheyne, for searching the Cochrane Stroke Register and assistance with developing and updating the search strategies. We also thank Hazel Fraser for assistance throughout the review process, co-authors of previous versions of this review, and Helen Lang for title and abstract screening. We thank Professor Stefano Ricci for translating a study in Italian, Mansur Kutlubaev for translating studies in Russian, Dr Matthias Volz and Simon
Ladwig for translating and extracting from a German study, Zien Zhou for translating letters for personal communication for studies in Chinese, and Dr Megumi Oshima and Dr Sohei Yoshimura for extracting data from a Japanese study. Special acknowledgement and thanks go to Dr Judith Redfern who led the screening, reviewing, and extracting of data for parts of this update but had to stop when she was diagnosed with terminal cancer. Jude died in February 2018. 


\section{R E F E R E N C E S}

\section{References to studies included in this review}

Almeida 2006 \{published data only\}

Almeida OP, Waterreus A, Hankey GJ. Preventing depression after stroke: results from a randomised placebo-controlled trial. Journal of Clinical Psychiatry 2006;67(7):1104-9.

\section{Creytens 1980 \{published data only\}}

Creytens G. New methods in treatment of cerebral pathology [Nouveautes dans le traitement de la pathologie cerebrale]. Acta Therapeutica 1980;6(1):33-53.

\section{Dam 1996a \{published data only\}}

Dam M, Tonin P, De Boni A, Pizzolato G, Casson S, Ermani M, et al. Effects of fluoxetine and maprotiline on functional recovery in poststroke hemiplegic patients undergoing rehabilitation therapy. Psychiatria Hungarica 1998;Suppl 2:47-54.

* Dam M, Tonin P, De Boni A, Pizzolato G, Casson S, Ermani M, et al. Effects of fluoxetine and maprotiline on functional recovery in poststroke hemiplegic patients undergoing rehabilitation therapy. Stroke 1996;27(7):1211-4.

\section{Dam 1996b \{published data only\}}

Dam M, Tonin P, De Boni A, Pizzolato G, Casson S, Ermani M, et al. Effects of fluoxetine and maprotiline on functional recovery in poststroke hemiplegic patients undergoing rehabilitation therapy. Psychiatria Hungarica 1998;Suppl 2:47-54.

* Dam M, Tonin P, De Boni A, Pizzolato G, Casson S, Ermani M, et al. Effects of fluoxetine and maprotiline on functional recovery in poststroke hemiplegic patients undergoing rehabilitation therapy. Stroke 1996;27(7):1211-4

\section{Forster 1996 \{published data only\}}

Dowswell G, Lawler J, Dowswell T, Young J, Forster A, Hearn J. Investigating recovery from stroke: a qualitative study. Journal of Clinical Nursing 2000;9:507-15.

Dowswell G, Lawler J, Hearn J, Young J, Forster A. Recovering after stroke: a qualitative study of patient and care-giver views and the impact of specialist nurse intervention. Research report to Northern and Yorkshire Regional Health Authority from the Department of Applied Social Studies, University of Bradford, Bradford, UK 1999.

Dowswell G, Lawler J, Young J, Forster A, Hearn J. A qualitative study of specialist nurse support for stroke patients and caregivers at home. Clinical Rehabilitation 1997;11:293-301.

Dowswell G, Lawler L, Young J. Unpacking the 'black box' of a nurse-led stroke support service. Clinical Rehabilitation 2000;14:160-71.

* Forster A, Young J. Specialist nurse support for patients with stroke in the community: a randomised controlled trial. BMJ 1996;312(7047):1642-6.

Lawler J, Dowswell G, Hearn J, Forster A, Young J. Recovering from stroke: a qualitative investigation of the role of goal setting in late stroke recovery. Journal of Advanced Nursing 1999;30(2):401-9.

Young J, Bogle S, Forster A. Determinants of social outcome measured by the Frenchay activities index at one year after stroke onset. Cerebrovascular Diseases 2001;12:114-20.

Young J, Forster A. A randomized trial of community stroke support. Clinical Rehabilitation 1996;10:353-4.

Goldberg 1997 \{published data only\}

Goldberg G, Segal ME, Berk SN, Schall RR, Gershkoff AM. Stroke transition after inpatient rehabilitation. Topics in Stroke Rehabilitation 1997;4(1):64-79.

Grade 1998 \{published data only\}

Grade C, Redford B, Chrostowski J, Toussaint L, Blackwell B. Methylphenidate in early poststroke recovery: a double-blind, placebo-controlled study. Archives of Physical Medicine and Rehabilitation 1998;79(9):1047-50.

\section{Hoffman 2015 \{published data only\}}

ACTRN12609000741280. Evaluation of brief interventions for enhancing early emotional adjustment following stroke: A pilot randomised controlled trial. https://www.anzctr.org.au/Trial/ Registration/TrialReview.aspx?id=308355\&isClinicalTrial=False (first received 25 August 2009).

* Hoffmann T, Ownsworth T, Eames S, Shum D. Evaluation of brief interventions for enhancing early emotional adjustment following stroke: a pilot randomised controlled trial. Topics in Stroke Rehabilitation 2015;22(2):117-26.

Ownsworth T, Hoffman T, Stemm B, Evans E, Howlett J, Shum D. Early cognitive appraisals, benefit finding and emotional status after stroke: pre-intervention associations and preliminary intervention findings. Brain Impairment 2011;12:66.

\section{House 2000 \{published data only\}}

Hill K, House A, Knapp P, Wardhaugh C, Bamford J, Vail A. Prevention of mood disorder after stroke: a randomised controlled trial of problem solving therapy versus volunteer support. BMC Neurology 2019;19(128):1-10.

House A, Knapp P, Dempster C, Garbett F, Todd C, Bamford J, et al. Does psychological treatment improve the outcomes after stroke? In: Proceedings of the British Psychological Society. Vol. 6. 1998:33.

* House A. Problem-solving therapy improves psychological outcome after stroke: a randomised controlled trial. Unpublished 2003.

House A. The treatment of depression after stroke. Journal of Psychosomatic Research 2000;48(3):235.

\section{Kerr 2018 \{published data only\}}

Kerr D, Mackey E, Wijeratne T, McCann T. Nursing early motivational interviewing on post stroke depressive symptoms: pilot randomized controlled trial of the good mood intervention program. International Journal of Stroke 2014;19:245. 
* Kerr D, McCann T, Mackey E, Wijeratne T. Effects of early motivational interviewing on post-stroke depressive symptoms: a pilot randomised study of the Good Mood Intervention program. International Journal of Nursing Practice 2018;24:1-8.

Palomaki 1999 \{published data only\}

Berg A, Palomaki H, Lehtihalmes M, Lonnqvist J, Kaste M. Poststroke depression: an 18-month follow-up. Stroke 2003;34:138-43.

Palomaki H, Kaste M, Berg A, Lonnqvist R, Lehtihalmes M. Poststroke sleep disorder treated with mianserin. Cerebrovascular Diseases 1997;7 Suppl 4:27.

Palomaki H, Kaste M, Berg A, Lonnqvist R, Lonnqvist J, Lehtihalmes $M$, et al. Complaints of poststroke insomnia and its treatment with mianserin. Cerebrovascular Diseases 2003;15(1-2):56-62.

* Palomaki H, Kaste M, Berg A, Lonnqvist R, Lonnqvist J, Lehtihalmes $\mathrm{M}$, et al. Prevention of poststroke depression: 1 year randomised placebo controlled double blind trial of mianserin with 6 month follow up after therapy. Journal of Neurology, Neurosurgery and Psychiatry 1999;66(4):490-4.

\section{Rasmussen 2003 \{published data only\}}

* Rasmussen A, Lunde M, Poulsen D, Sorensen K, Qvitzau S, Bech P. A double-blind, placebo-controlled study of sertraline in the prevention of depression in stroke patients. Psychosomatics 2003;44(3):216-21.

Rasmussen A, Lunde M, Sorensen K, Qvitzau S, Bech P. A double-blind placebo-controlled study of sertraline in the prevention of depression in stroke patients. European Neuropsychopharmacology 2002;12 Suppl 3:231.

Rasmussen A. A double blind controlled study of the influence of sertraline on the incidence of post stroke depression and on emotional lability. PhD Thesis 2001.

Rasmussen A. Depression and stroke. Nordic Journal of Psychiatry 2001;55(4):288.

Rasmussen A. Prophylatic treatment for post stroke depression and comorbidity. Journal of Psychosomatic Research 2000;48(3):66.

\section{Reding 1986 \{published and unpublished data\}}

Reding MJ, Orto LA, Winter SW, Fortuna IM, Di Ponte P, McDowell FH. Antidepressant therapy after stroke: a doubleblind trial. Archives of Neurology 1986;43(8):763-5.

\section{Robinson 2000a \{published data only\}}

Chan KL, Campayo A, Moser DJ, Arndt S, Robinson RG. Aggressive behavior in patients with stroke: association with psychopathology and results of antidepressant treatment. Archives of Physical Medicine and Rehabilitation 2006;87:793-8.

Chemerinski E, Robinson RG, Arndt S, Kosier JT. The effect of remission of poststroke depression on activities of daily living in a double-blind randomized treatment study. Journal of Nervous and Mental Disease 2001;189(7):421-5.
Dean CE, Sonis J. Mortality and poststroke depression. American Journal of Psychiatry 2004;161(8):1506-7.

Franco K, Malhotra S. Poststroke depression. American Journal of Psychiatry 2001;158(4):658-9.

Jorge R. The effect of antidepressants on mortality after stroke. In: Proceedings of the 158th Annual Meeting of the American Psychiatric Association. USA, Atlanta, Georgia, 21-26 May 2005:16D

Jorge RE, Robinson RG, Arndt S, Starkstein S. Mortality and poststroke depression: a placebo-controlled trial of antidepressants. American Journal of Psychiatry 2003;160(10):1823-9.

Kimura M, Robinson RG, Kosier JT. Treatment of cognitive impairment after poststroke depression: a double-blind treatment trial. Stroke 2000;31(7):1482-6.

Kimura M, Tateno A, Robinson RG. Treatment of poststroke generalized anxiety disorder comorbid with poststroke depression: merged analysis of nortriptyline trials. American Journal of Geriatric Psychiatry 2003;11:320-7.

Narushima K, Chan KL, Kosier JT, Robinson RG. Does cognitive recovery after treatment of poststroke depression last? A 2year follow-up of cognitive function associated with poststroke depression. American Journal of Psychiatry 2003;160(6):1157-62.

Narushima K, Kosier JT, Robinson RG. Preventing poststroke depression: a 12-week double-blind randomized treatment trial and 21-month follow-up. Journal of Nervous and Mental Diseases 2002;190(5):296-303.

Narushima K, Robinson RG. The effect of early versus late antidepressant treatment on physical impairment associated with poststroke depression: is there a time-related therapeutic window? Journal of Nervous and Mental Disease 2003;191(10):645-52.

Robinson RG, Narushima K. Prevention strategies for post-stroke depression. Journal of Clinical Psychiatry 2002;63(11):1079.

* Robinson RG, Schultz SK, Castillo C, Kopel T, Kosier JT, Newman RM, et al. Nortriptyline versus fluoxetine in the treatment of depression and in short-term recovery after stroke: a placebo-controlled, double-blind study. American Journal of Psychiatry 2000;157(3):351-9.

Starkstein SE, Manes F. Apathy and depression following stroke. CNS Spectrums 2000;5(3):43-50.

Robinson 2000b \{published data only\}

Chan KL, Campayo A, Moser DJ, Arndt S, Robinson RG. Aggressive behavior in patients with stroke: association with psychopathology and results of antidepressant treatment. Archives of Physical Medicine and Rehabilitation 2006;87:793-8.

Chemerinski E, Robinson RG, Arndt S, Kosier JT. The effect of remission of poststroke depression on activities of daily living in a double-blind randomized treatment study. Journal of Nervous and Mental Disease 2001;189(7):421-5. 
Dean CE, Sonis J. Mortality and poststroke depression. American Journal of Psychiatry 2004;161(8):1506-7.

Franco K, Malhotra S. Poststroke depression. American Journal of Psychiatry 2001;158(4):658-9.

Jorge R. The effect of antidepressants on mortality after stroke. In: Proceedings of the 158th Annual Meeting of the American Psychiatric Association. USA, Atlanta, Georgia, 21-26 May 2005:16D.

Jorge RE, Robinson RG, Arndt S, Starkstein S. Mortality and poststroke depression: a placebo-controlled trial of antidepressants. American Journal of Psychiatry 2003;160(10):1823-9.

Kimura M, Robinson RG, Kosier JT. Treatment of cognitive impairment after poststroke depression: a double-blind treatment trial. Stroke 2000;31(7):1482-6.

Kimura M, Tateno A, Robinson RG. Treatment of poststroke generalized anxiety disorder comorbid with poststroke depression: merged analysis of nortriptyline trials. American Journal of Geriatric Psychiatry 2003;11(3):320-7.

Narushima K, Chan KL, Kosier JT, Robinson RG. Does cognitive recovery after treatment of poststroke depression last? A 2year follow-up of cognitive function associated with poststroke depression. American Journal of Psychiatry 2003;160(6):1157-62.

Narushima K, Kosier JT, Robinson RG. Preventing poststroke depression: a 12-week double-blind randomized treatment trial and 21-month follow-up. Journal of Nervous and Mental Diseases 2002;190(5):296-303.

Narushima K, Robinson RG. The effect of early versus late antidepressant treatment on physical impairment associated with poststroke depression: is there a time-related therapeutic window? Journal of Nervous and Mental Disease 2003;191(10):645-52.

Robinson RG, Narushima K. Prevention strategies for post-stroke depression. Journal of Clinical Psychiatry 2002;63(11):1079.

* Robinson RG, Schultz SK, Castillo C, Kopel T, Kosier JT, Newman RM, et al. Nortriptyline versus fluoxetine in the treatment of depression and in short-term recovery after stroke: a placebo-controlled, double-blind study. American Journal of Psychiatry 2000;157(3):351-9.

Starkstein SE, Manes F. Apathy and depression following stroke. CNS Spectrums 2000;5(3):43-50.

\section{Robinson 2008 \{published data only\}}

Robinson RG, Jorge RE, Moser DJ. Escitalopram and problemsolving therapy for prevention of post-stroke depression: a randomized controlled trial. JAMA 2008;299(20):2391-400.

\section{Roh 1996 \{published data only\}}

Roh JK, Lee KH, Kim M, Yamamoto M. Accelerated recovery from ischemic stroke with indeloxazine hydrochloride: results of a double-masked clinical study in Korea. Current Therapeutic Research, Clinical and Experimental 1996;57(8):632-42.

\section{Tsai 2011 \{published data only\}}

Tsai CS, Wua CL, Chouf SY, Tsanga HY, Su JA. Prevention of poststroke depression with milnacipran in patients with acute ischemic stroke: a double-blind randomized placebocontrolled trial. International Clinical Psychopharmacology 2011;26(5):263-7.

Watkins 2007 \{published and unpublished data\}

Deans CF, Jack CIA. Evaluation of motivational interviewing early after acute stroke: a randomized controlled trial. Clinical Rehabilitation 2006;20:731-6.

Sutton C, Dickinson H, Leathley M, Hills K, Auton M, Lightbody E, et al. Motivational interviewing: altering outcome after stroke. In: 12th European Stroke Conference. Valencia, Spain, 2003 May 21-24:103.

Van den Broek MD. Motivational interviewing after stroke. In: Society for Research in Rehabilitation Winter Conference. Preston, United Kingdom, 2003.

* Watkins CL, Auton MF, Deans CF, Dickinson HA, Jack Cl, Lightbody $\mathrm{CE}$, et al. Motivational interviewing early after acute stroke: a randomized, controlled trial. Stroke 2007;38:1004-9. [ISRCTN544654472]

\section{Wichowicz 2017 \{published data only\}}

Wichowicz HM, Puchalska L, Rybak-Korneluk AM, Gąsecki D, Wiśniewska A. Application of Solution-Focused Brief Therapy (SFBT) in individuals after stroke. Brain Injury 2017;31(11):1507-12.

\section{Xu 2006 \{published data only\}}

Xu J, Wang JP, Liu J. Preventive effects of antidepressant on post-stroke depression. Chinese Mental Health Journal 2006;20(3):186-8.

\section{References to studies excluded from this review}

\section{Aben 2014 \{published data only\}}

Aben L, Heijenbrok-Kal MH, Ponds RW, BusschbachJJV, Ribbers GM. Long-lasting effects of a new memory self-efficacy training for stroke patients: a randomized controlled trial. Neurorehabilitation and Neural Repair 2014;28(3):199-206.

* Aben L, Heijenbrok-Kal MH, van Loon EM, Groet E, Ponds RW, Busschbach JJV, et al. Training memory self-efficacy in the chronic stage after stroke: a randomized controlled trial. Neurorehabilitation and Neural Repair 2013;27(2):110-7.

\section{Agnoli 1985 \{published data only\}}

Agnoli A, Fioravanti M, Lechner H. Efficacy of CDP-choline in chronic cerebral vascular diseases (CCVD). In: Zappia V, Kennedy EP, Nilsson BI, Galletti P, editors(s). Novel Biochemical, Pharmacological and Clinical Aspects of Cytidinediphosphocholine. New York: Elsevier, 1985:305-15.

Bramanti 1989 \{published data only\}

Bramanti P, Ricci RM, Di Bella P, De Luca GP, Sessa E, Di Leo $M$, et al. Neuropsychologic and clinical evaluation of the 
administration of protirelin tartrate in cerebrovascular diseases. Rassegna di Medicina Interna 1989;10(4):157-61.

\section{Casella 1960 \{published data only\}}

Casella C, Sokolow J. A study to determine the energizing effects of iproniazid (Marsilid) on a group of hemiplegics. Archives of Physical Medicine and Rehabilitation 1960;41:381-5.

Choi-Kwon 2006 \{published data only\}

Choi-Kwon S, Choi J, Kwon SU, Kang D, Kim JS. Fluoxetine is not effective in the treatment of poststroke fatigue: a doubleblind, placebo controlled study. Cerebrovascular Diseases 2007;23:103-8.

* Choi-Kwon S, Han SW, Kwon SU, Kang DW, Choi JM, Kim JS. Fluoxetine treatment in poststroke depression, emotional incontinence, and anger proneness: a double-blind, placebocontrolled study. Stroke 2006;37:156-61.

Kim JS. Post-stroke emotional disturbances. In: Symposium 3: Prevention of Recurrent Vascular Events and Other Complications of Stroke. 2006.

\section{Downes 1995 \{published data only\}}

Downes B, Rooney V, Oyebode JR, Roper-Hall A, Mayer P, Main A. The effect of giving information and counselling on depression and anxiety in stroke survivors and carers (The Birmingham Stroke Counselling Project). Unpublished 1995.

\section{F.X. 1976 \{published data only\}}

FX Project for Phase III Study. Double blind study of FX-505 (ifenprodil) on cerebrovascular diseases: phase III study. Rinsho Hyoka 1976;4(3):419-58.

\section{Friedland 1992 \{published data only\}}

Friedland J, McColl M. Social support for stroke survivors: development and evaluation of an intervention program. Physical and Occupational Therapy in Geriatrics 1989;7(3):55-69.

* Friedland JF, McColl M. Social support intervention after stroke: results of a randomized trial. Archives of Physical Medicine and Rehabilitation 1992;73(6):573-81.

Graffingo 2003 \{published data only\}

Graffingo C. Poststroke depression and functional recovery (SADBRAIN). Unpublished 2003.

\section{Hadidi 2014 \{published data only\}}

Hadidi N, Buckwalter K, Lindquist R, Rangen C. Feasibility of a pilot study of problem-solving therapy for stroke survivors. Rehabilitation Nursing 2014;40(5):327-37.

\section{ljzerman 2005 \{published data only\}}

ISRCTN88489864. Influence of a single dose of fluoxetine on muscle activation patterns and functional ability in chronic stroke patients. http://www.isrctn.com/ISRCTN88489864? $q=\&$ filters=\&sort=\&offset=14788\&totalResults=18648\&page=296\&pag search (first received 20 December 2005).

ljzerman MJ, van Genderen HI, Nijlant JJ. Effect of a single dose of the SSRI fluoxetine on motor activation patterns of the upper extremity in chronic stroke survivors. Neurorehabilitation and Neural Repair 2006;20(1):158 (Abst P2-024).

Isenberg 2000 \{published data only\}

Isenberg N. A double-blind, placebo controlled, dose-ranging study of nefiracetam in patients with post-stroke depression. Daiichi Pharmaceutical Co 2000.

Kim 2017 \{published data only\}

Kim JS, Lee EJ, Chang D, Park JH, Ahn SH, Cha JK, et al, EMOTION investigators. Efficacy of early administration of escitalopram on depressive and emotional symptoms and neurological dysfunction after stroke: a multicentre,doubleblind,randomised, placebo-controlled study. Lancet Psychiatry 2017;4(1):33-41.

Kim 2017a \{published data only\} Kim KU, Kim SH, An TG. The effects of repetitive transcranial magnetic stimulation (rTMS) on depression, visual perception, and activities of daily living in stroke patients. Journal of Physical Therapy Science 2017;29(6):1036-9.

Laska 2005 \{published data only\}

Laska AC, Mårtensson B, Kahan T, von Arbin M, Murray V. Recognition of depression in aphasic stroke patients. Cerebrovascular Diseases 2007;24(1):74-9.

* Laska AC, von Arbin M, Kahan T, Hellblom A, Murray V. Longterm antidepressant treatment with moclobemide for aphasia in acute stroke patients: a randomised, double-blind, placebocontrolled study. Cerebrovascular Diseases 2005;19(2):125-32.

\section{Latow 1983 \{published data only\}}

Latow J. Psychotherapy and its effect on depression, sickrole identification and rehabilitation outcome for stroke victims. Archives of Physical Medicine and Rehabilitation 1983;64(10):511-2.

\section{Leathley 2003 \{published data only\}}

Leathley M, Fall S, Sharma A, Watkins C, Barer D. Life after stroke: multicentre trial of psychosocial interventions after stroke. Cerebrovascular Diseases 2003;16 Suppl 4:70.

\section{Leijon 1989 \{published data only\}}

Leijon G, Boivie J. Central post-stroke pain: a controlled trial of amitriptyline and carbamazepine. Pain 1989;36(1):27-36.

\section{Lobjanidze 2010 \{published data only\}}

Lobjanidze N, Dzagnidze A, Jeiranashvili A, Kukava M, Beridze M, Khachiashvili $\mathrm{M}$, et al. Long-term effects of the use of citicoline (ceraxone) in the post-stroke cognitive-mood impairment. Cerebrovascular Diseases 2010;29 Suppl 2:615.

Martucci 1986 \{published data only\}

Martucci N, Manna V, Mailland F. Electroencephalographicpharmacological and neuropsychological study of dihydroergocristine mesylate in patients with chronic cerebrovascular disease. Advances in Therapy 1986;3(4):210-23. 
McCafferty 2000 \{published data only\}

McCafferty G, Kilcullen P, Kligerman B, Hickling E, Taylor A, Davidson B. A psychosocial intervention for post-stroke depression (PDS): a controlled treatment study. Journal of Stroke and Cerebrovascular Diseases 2000;9(4):200.

Ohtomo 1985 \{published data only\}

Ohtomo E, Kutsuzawa T, Araki G, Hirai S, Terashi A, Kuzuya F, et al. Clinical usefulness of tiapride on psychiatric symptoms caused by cerebrovascular disorders. Clinical Evaluation 1985;13:295-332.

\section{Ostwald 2006 \{published data only\}}

NCT00178529. Intervention for stroke survivors and spousal caregivers. https://clinicaltrials.gov/ct2/show/NCT00178529 (first received 15 September 2005).

* Ostwald SK, Wasserman J, Davis S. Medications, comorbidities, and medical complications in stroke survivors: the CAReS study. Rehabilitation Nursing 2006;31(1):10-4.

\section{Ostwald 2014 \{published data only\}}

NCT00178529. Intervention for stroke survivors and spousal caregivers. https://clinicaltrials.gov/ct2/show/NCT00178529 (first received 15 September 2005).

* Ostwald S, Godwin K, Cron S, Kelley C, Hersch G, Davis S. Home-based psychoeducational and mailed information programs for stroke-caregiving dyads post-discharge: a randomized trial. Disability \& Rehabilitation 2014;36(1):55-62.

Ostwald SK, Davis S, Hersch G, Kelley C, Godwin KM. Evidence based educational guidelines for stroke survivors after discharge home. Journal of Neuroscience Nursing 2008;40(3):173-91.

Ostwald SK, Wasserman J, Davis S. Medications, comorbidities, and medical complications in stroke survivors: the CAReS study. Rehabilitation Nursing 2006;31(1):10-4.

\section{Otomo 1986 \{published data only\}}

Otomo E, Tohgi H, Hirai S, Gotoh F, Hasegawa K, Tazaki Y, et al. Clinical evaluation of YM-08054 (indeloxazine) in the treatment of cerebrovascular disorder. Igaku no Ayumi 1986;136(7):535-55.

\section{Poppitt 2009 \{published data only\}}

Poppitt SD, Howe CA, Lithander FE, Silvers KM, LinRB, Croft J, et al. Effects of moderate-dose omega-3 fish oil on cardiovascular risk factors and mood after ischemic stroke: a randomized controlled trial. Stroke 2009;40:3485-92.

\section{Raffaele 1996 \{published data only\}}

Raffaele R, Rampello L, Vecchio I, Tornali C, Malaguanera M. Trazodone therapy of the post-stroke depression. Archives of Gerontology and Geriatrics 1996;22 Suppl 1:217-20.

\section{Sasaki 2017 \{published data only\}}

Sasaki N, Hara T, Yamada N, Niimi M, Kakuda W, Abo M. The efficacy of high-frequency repetitive transcranial magnetic stimulation for improving apathy in chronic stroke patients. European Neurology 2017;78:28-32.
Sivenius 2001 \{published data only\}

Sivenius J, Sarasoja T, Aaltonen H, Heinonen E, Kilkku O, Reinikainen K. Selegiline treatment facilitates recovery after stroke. Journal of Neurologic Rehabilitation 2001;15(3):183-90.

Walker-Batson 1995 \{published data only\}

Walker-Batson D, Smith P, Curtis S, Unwin H, Greenlee R. Amphetamine paired with physical therapy accelerates motor recovery after stroke: further evidence. Stroke 1995;26(12):2254-9.

\section{Yamamoto 1999 \{published data only\}}

Yamamoto T, Nishimura R, Takagi T. Importance of a psychological approach to the chronic cerebral vascular accident patient. In: Proceedings of the 13th International Congress of the World Confederation of Physical Therapy. Yokohama, Japan, 1999.

\section{References to studies awaiting assessment}

\section{Chang 2011 \{published data only\}}

Chang K, ZhangH, Xia Y, Chen C. Testing the effectiveness of knowledge and behavior therapy in patients of hemiplegic stroke. Topics in Stroke Rehabilitation 2011;18(5):525-35.

\section{Evans 1985 \{published data only\}}

Evans RL, Kleinman L, Halar EM, Herzer K. Predicting outcome of group counselling with severely disabled patients. American Journal of Physical Medicine 1985;64(1):24-31.

\section{IRCT201112228490N1 \{published data only\}}

IRCT201112228490N1. Effect of fluoxetine on functional recovery of patients with cerebrovascular accident following middle cerebral artery trunk obstruction: a randomized clinical trial. http://en.irct.ir/trial/8954 (first received 25 January 2012).

Katz 1998 \{published data only\}

Katz RA, Hubbard DJ, Blaine J. The effect of group psychotherapy on post-stroke depression. Rehabilitation Psychology 1998;43(2):178.

\section{Razazian 2016 \{published data only\}}

Razazian N, Esmaeili O, Almasi A. Effect of fluoxetine on motor improvement in ischemic stroke patients: a double blind clinical trial study. Journal of Research in Medical Science 2016; In press:1-6.

\section{References to ongoing studies}

Kirkevold 2018 \{published data only\}

Kirkevold M, Bragstad LK, Bronken BA, Kvigne K, Martinsen R, Hjelle EG, et al. Promoting psychosocial well-being following stroke: study protocol for a randomized, controlled trial. BMC Psychology 2018;6(12):1-12.

Sitzer 2002 \{published data only\}

Huff W. Prevention of depression after ischemic stroke: PreDIS. Stroke Trials Directory, www.strokecenter.org/trials/TrialDetail (first received 25 September 2001). 
Huff W, Steckel R, Sitzer M. Prevention of depression after ischemic stroke - the PreDIS study. Pharmacopsychiatry 2001;34(5):Abst. 85.

* Sitzer M, Steckel R. Prevention of poststroke depression after acute ischaemic stroke using the selective serotonin reuptakeinhibitor sertraline (PREDIS-Study). Stroke 2002;33(2):651-2.

Sitzer M, Steckel R. Prevention of poststroke depression after acute ischemic stroke using the selective serotonin reuptakeinhibitor sertraline (PreDIS-study). In: Proceedings of the 27th International Stroke Conference. USA, San Antonio: The American Stroke Association, 7-9 February 2002:(Abst. CTP326).

\section{Additional references}

\section{Adams 1990}

Adams RJ, Meador KJ, Sethi KD, Grotta JC, Thomson DS. Graded neurologic scale for use in acute hemispheric stroke treatment protocols. Stroke 1990;71:876-80.

\section{Allida 2020}

Allida S, Cox KL, Hsieh CF, Lang H, House A, Hackett ML. Pharmacological, psychological, and non-invasive brain stimulation interventions for treating depression after stroke. Cochrane Database of Systematic Reviews 2020, Issue 1. [DOI: 10.1002/14651858.CD003437.pub4]

\section{Anderson 1995}

Anderson CS, Linto J, Stewart-Wynne EG. A population-based assessment of the impact and burden of caregiving for longterm stroke survivors. Stroke 1995;26:843-9.

\section{APA 1987}

American Psychiatric Association. Diagnostic and statistical manual of mental disorders: DSM-III-R. Washington, DC: American Psychiatric Association, 1987.

\section{APA 1994}

American Psychiatric Association. Diagnostic and statistical manual of mental disorders: DSM-IV. Washington, DC: American Psychiatric Association, 1994.

\section{APA 2013}

American Psychiatric Association, DSM-5 Task Force. Diagnostic and statistical manual of mental disorders: DSM- $5^{\mathrm{TM}}$ (5th ed.). Arlington, VA, USA: American Psychiatric Publishing, Inc., 2013.

\section{Atkins 2004}

Atkins D, Best D, Briss PA, Eccles M, Falck-Yitter Y, Flottorp G, et al. GRADE Working Group. Grading quality of evidence and strength of recommendations. BMJ 2004;328(7454):1490.

\section{Ayerbe 2013}

Ayerbe L, Ayis S, Wolfe CDA, Rudd AG. Natural history, predictors and outcomes of depression after stroke: systematic review and meta-analysis. British Journal of Psychiatry 2013;202:14-21.

\section{Baker 2018}

Baker C, Worrall L, Rose M, Hudson K, Ryan B, O'Byrne L. A systematic review of rehabilitation interventions to prevent and treat depression in post-stroke aphasia. Disability and Rehabilitation 2018;40(16):1870-92.

\section{Beck 1961}

Beck AT, Ward C, Mendelson M. An inventory for measuring depression. Archives of General Psychiatry 1961;4:561-71.

\section{Clark 1999}

Clark HD, Wells GA, Huët C, McAlister FA, Salmi LR, Fergusson D, et al. Assessing the quality of randomized trials: reliability of the Jadad scale. Control Clinical Trials 1999;20(5):448-52.

\section{Deeks 2011}

Deeks JJ, Higgins JP, Altman DG, editor(s). Chapter 9: Analysing data and undertaking meta-analyses. In: Higgins JP, GreenS, editor(s). Cochrane Handbook for Systematic Reviews of Interventions Version 5.1.0 (updated March 2011). The Cochrane Collaboration, 2011. Available from handbook.cochrane.org.

\section{Ebrahim 1987}

Ebrahim S, Barer D, Nouri F. Affective illness after stroke. British Journal of Psychiatry 1987;151:52-6.

\section{FOCUS 2019}

FOCUS trial collaboration. Effects of fluoxetine on functional outcomes after acute stroke (FOCUS): a pragmatic, doubleblind, randomised, controlled trial. Lancet 2011;393:265-74.

\section{Folstein 1975}

Folstein MF, Folstein SE, McHugh PR. 'Mini-Mental State': a practical method for grading the cognitive state of patients for the clinician. Journal of Psychiatric Research 1975;12:189-98.

\section{Goldberg 1972}

Goldberg DP. The Detection of Psychiatric Illness by Questionnaire. Vol. Maudsley Monograph No. 21. Oxford: Oxford University Press, 1972.

\section{Gompertz 1993}

Gompertz P, Pound P, Ebrahim S. The reliability of stroke outcome measurement. Clinical Rehabilitation 1993;7:290-6.

\section{GRADEproGDT 2015 [Computer program]}

McMaster University (developed by Evidence Prime). Available at gradepro.org GRADEproGDT. Hamilton (ON): McMaster University (developed by Evidence Prime). Hamilton (ON): McMaster University (developed by Evidence Prime). Available at gradepro.org, 2015.

\section{Hackett 2005a}

Hackett ML, Yapa C, Parag V, Anderson CS. Frequency of depression after stroke: a systematic review of observational studies. Stroke 2005;36:1330-40.

\section{Hackett 2014}

Hackett M, Pickles K. Part I: frequency of depression after stroke: an updated systematic review and meta-analysis of observational studies. World Stroke Organisation 2014;9(December):1017-25 


\section{Hamilton 1960}

Hamilton M. Rating scale for depression. Journal of Neurology, Neurosurgery and Psychiatry 1960;23:56-62.

\section{Higgins 2019}

Higgins JP, Thomas J, Chandler J, Cumpston M, Li T, Page MJ, et al (editors). Cochrane Handbook for Systematic Reviews of Interventions Version 6.0 (updated July 2019). Cochrane, 2019. Available from www.training.cochrane.org/handbook.

\section{House 1989}

House A, Dennis M, Hawton K, Warlow C. Methods of identifying mood disorders in stroke patients: experience in the Oxfordshire community Stroke Project. Age and Aging 1989;18:371-9.

\section{House 2001}

House A, Knapp P, Bamford J, Vail A. Mortality at 12 and 24 months after stroke may be associated with depressive symptoms at 1 month. Stroke 2001;32:696-701.

\section{Hunt 1986}

Hunt SM, McEwan J, McKenna SP. Measuring Health Status. Beckenham: Croom Helm, 1986.

\section{ICD 101992}

World Health Organization. The ICD-10 classification of mental and behavioural disorders: clinical descriptions and diagnostic guidelines. Geneva: World Health Organization, 1992.

\section{Katona 1995}

Katona CL, Watkin V. Depression in old age. Reviews in Clinical Gerontology 1995;5:427-41.

\section{Lewis 2019}

Lewis G, Duffy L, Ades A, Amos R, Araya R, Brabyn S, et al. The clinical effectiveness of sertraline in primary care and the role of depression severity and duration (PANDA): a pragmatic, doubleblind, placebo-controlled randomised trial. Lancet Psychiatry 2019;6(11):903-14.

\section{Mahoney 1965}

Mahoney FI, Barthel DW. Functional evaluation: the Barthel Index. Maryland State Medical Journal 1965;14:61-5.

\section{Montgomery 1979}

Montgomery SA, Asberg M. A new depression scale designed to be sensitive to change. British Journal of Psychiatry 1979;134:382-9.

\section{Morris 1993}

Morris PL, Robinson RG, Andrzejewski P, Samuels J, Price TR. Association of depression with 10-year poststroke mortality. American Journal of Psychiatry 1993;150:124-9.

\section{Normand 1999}

Normand ST. Meta-analysis: formulating, evaluating,combining, and reporting. Statistics in Medicine 1999;18:321-59.

\section{Parikh 1990}

Parikh RM, Robinson RG, Lipsey JR, Starkstein SE, Fedoroff JP, Price TR. The impact of poststroke depression on recovery in activities of daily living over a 2-year follow-up. Archives of Neurology 1990;47:785-9.

\section{Rachid 2018}

Rachid F. Maintenance repetitive transcranial magnetic stimulation (rTMS) for relapse prevention in with depression: a review. Psychiatry Research 2018;262:363-72.

\section{Rankin 1957}

Rankin J. Cerebral vascular accidents in people over the age of 60. II. Prognosis. Scottish Medical Journal 1957;2:200-215.

\section{Review Manager 2014 [Computer program]}

Nordic Cochrane Centre, The Cochrane Collaboration Nordic Cochrane Centre, The Cochrane Collaboration. Review Manager 5 (RevMan 5). Version 5.3. Copenhagen: Nordic Cochrane Centre, The Cochrane Collaboration, 2014.

\section{Robinson 1986}

Robinson RG, Bolla-Wilson K, Kaplan E, Lipsey JR, Price TR. Depression influences intellectual impairment in stroke patients. British Journal of Psychiatry 1986;148:541-7.

\section{Salter 2013}

Salter KL, Foley NC, Zhu L, Jutai JW, Teasell RW. Prevention of poststroke depression: Ddes prophylactic pharmacotherapy work? Journal of Stroke and Cerebrovascular Diseases 2013;22(8):1243-51.

\section{Schünemann 2019}

Schünemann HJ, Higgins JP, Vist GE, Glasziou P, Akl EA, Skoetz N, et al. Chapter 14: Completing 'Summary of findings' tables and grading the certainty of the evidence. In: Higgins JP, Thomas J, Chandler J, Cumpston M, Li T, Page MJ, et al (editors). Cochrane Handbook for Systematic Reviews of Interventions Version 6.0 (updated July 2019). Available from www.training.cochrane.org/handbook.

\section{Sinyor 1986}

Sinyor D, Amato P, Kaloupek DG, Becker R, Goldenberg M, Coopersmith H. Post-stroke depression: relationships to functional impairment, coping strategies, and rehabilitation outcome. Stroke 1986;17:1102-7.

\section{Stenager 1998}

Stenager EN, Madsen C, Stenager E, Boldsen J. Suicide in patients with stroke: epidemiological study. BMJ 1998;316:1206.

\section{Turner-Stokes 2003}

Turner-Stokes L. Poststroke depression: getting the full picture. Lancet 2003;361:1757-8.

\section{Verhagen 2001}

Verhagen AP, de Vet $\mathrm{HC}$, de Bie RA, Boers M, van den Brandt PA. The art of quality assessment of RCTs included in systematic reviews. Journal of Clinical Epidemiology 2001;54:651-4.

\section{Wade 1985}

Wade DT, Legh-Smith J, Langton Hewer R. Social activities after stroke: measurement and natural history using the 
Frenchay Activities Index. International Rehabilitation Medicine 1985;7(4):176-81.

\section{Ware 1993}

Ware JE, Snow KK, Kosinski M, Gandek B. SF-36 HealthSurvey: manual and interpretation guide. Boston, Mass:New England Medical Center, Health Institute 1993.

\section{WHO 2000}

World Health Organization. The World Health Report 2000: Health systems: improving performance. Geneva: World Health Organization, 2000.

\section{Yi 2010}

Yi ZM, Liu F Zhai SD. Fluoxetine for the prophylaxis of poststroke depression in patients with stroke: a meta-analysis. International Journal of Clinical Practice 2010;64(9):1310-7.

\section{Zigmond 1983}

Zigmond AS, Snaith RP. The Hospital Anxiety and Depression Scale. Acta Psychiatrica Scandinavica 1983;67:361-70.

\section{Zimmerman 2002}

Zimmerman M, Mattia JI, Posternak MA. Are subjects in pharmacological treatment trials of depression representative of patients in routine clinical practice? American Journal of Psychiatry 2002;159:469-73.

\section{CHARACTERISTICS OF STUDIES}

Characteristics of included studies [ordered by study ID]

\section{References to other published versions of this review} Anderson 2001

Anderson CC, Hackett ML, House AA. Interventions for preventing depression after stroke. Cochrane Database of Systematic Reviews 2001, Issue 1. [DOI: 10.1002/14651858.CD003689]

\section{Anderson 2004}

Anderson CS, Hackett ML, House AO. Interventions for preventing depression after stroke. Cochrane Database of Systematic Reviews 2004, Issue 2. [DOI: 10.1002/14651858.CD003689.pub2]

\section{Hackett 2005}

Hackett ML, Anderson CS, House AO. Management of depression after stroke: a systematic review of pharmacological therapies. Stroke 2005;36:1092-7.

\section{Hackett 2008}

Hackett ML, Anderson CS, House A, Halteh C. Interventions for preventing depression after stroke. Cochrane Database of Systematic Reviews 2008, Issue Issue 3. [DOI: 10.1002/14651858.CD003689.pub3]

* Indicates the major publication for the study

Almeida 2006

\section{Study characteristics}

\begin{tabular}{|c|c|}
\hline \multirow[t]{4}{*}{ Methods } & Study design: parallel design \\
\hline & Number of arms: 2 \\
\hline & Treatment arm: sertraline (SSRI) \\
\hline & Control arm: matched placebo \\
\hline \multirow[t]{8}{*}{ Participants } & Geographical location: Australia \\
\hline & Setting: inpatient \\
\hline & Stroke criteria: acute ischaemic or haemorrhagic stroke \\
\hline & $\begin{array}{l}\text { Method of stroke diagnosis: via clinical signs (ICD-10) and CT (100\% imaged, 10/111 CT scan did not } \\
\text { show acute ischaemia) }\end{array}$ \\
\hline & $\begin{array}{l}\text { Time since stroke: stroke on average }<2 \text { weeks prior to randomisation } \\
\text { Inclusion criteria: not reported }\end{array}$ \\
\hline & $\begin{array}{l}\text { Exclusion criteria: 1) severe communication difficulties; 2) unstable medical condition; 3) severe cogni- } \\
\text { tive impairment and depression; 4) taking antidepressants within } 4 \text { weeks of stroke; 5) contraindication } \\
\text { to sertraline }\end{array}$ \\
\hline & Depression criteria: HADS score $>8$ \\
\hline & Total number randomised in this trial: 111 \\
\hline
\end{tabular}


Almeida 2006 (Continued)

Number randomised to treatment group: 55 (67\% men, mean age 68 years, SD 13)

Number randomised to control group: 56 (62\% men, mean age 67 years, SD 13)

Total number included in the final analysis: 99

Number included in treatment group for final analysis: 48

Number included in control group for final analysis: 51

$\begin{array}{ll}\text { Interventions } & \text { Treatment: sertraline (SSRI), } 50 \mathrm{mg} \text {, daily (night) } \\ \text { Control: matched placebo } \\ \text { Treatment duration: treatment continued for } 24 \text { weeks } \\ \text { Follow-up: } 28 \text { weeks post treatment end ( } 52 \text { weeks from baseline) }\end{array}$

\begin{tabular}{ll}
\hline Outcomes & Primary outcomes \\
- Depression measured using the HDRS \\
Secondary outcomes \\
- Cognitive function measured using the MMSE \\
- Disability measured using the mRS
\end{tabular}

Notes

\section{Risk of bias}

\begin{tabular}{lll}
\hline Bias & Authors' judgement & Support for judgement \\
\hline $\begin{array}{l}\text { Random sequence genera- } \\
\text { tion (selection bias) }\end{array}$ & Low risk & $\begin{array}{l}\text { Quote: "Subjects were allocated to 24-week treatment with placebo or sertra- } \\
\text { line (fixed daily dose of 50mg at night) according to a computer-generated ran- } \\
\text { dom list of numbers ..." pp. 1105 }\end{array}$ \\
\hline $\begin{array}{l}\text { Allocation concealment } \\
\text { (selection bias) }\end{array}$ & Low risk & $\begin{array}{l}\text { Quote: "Placebo and sertraline were delivered in capsules that had the same } \\
\text { size, shape, colour, smell, and weight" pp. 1105 }\end{array}$ \\
\hline $\begin{array}{l}\text { Blinding of participants } \\
\text { and personnel (perfor- } \\
\text { mance bias) } \\
\begin{array}{l}\text { All outcomes } \\
\text { L }\end{array}\end{array}$ & Low risk & $\begin{array}{l}\text { Quote: "Both the research team and participants were unaware of treatment } \\
\text { allocation ..." pp. 1105 }\end{array}$ \\
\hline
\end{tabular}

Blinding of outcome as- Unclear risk sessment (detection bias)

All outcomes
Quote: "We designed this randomized, double-blind, placebo control trial ..." pp. 1105

$\begin{array}{ll}\begin{array}{l}\text { Incomplete outcome data } \\ \text { (attrition bias) }\end{array} & \text { High risk } \\ \text { All outcomes } & \begin{array}{l}\text { Quote: "... last observation carried forward (LOCF) analyses to investigate the } \\ \text { primary endpoint of interest for this study ..." pp. } 1106\end{array} \\ & \begin{array}{l}\text { Comments: per protocol analysis presented. 11/111 were not included in the } \\ \text { analysis }\end{array}\end{array}$

Selective reporting (re- Unclear risk Comments: no trial protocol available to compare with the publication
porting bias)

\begin{tabular}{|c|c|c|}
\hline Other bias & Unclear risk & $\begin{array}{l}\text { Comments: more participants in treatment group with previous heart attack } \\
\text { and stroke, also higher levels of hypertension }\end{array}$ \\
\hline
\end{tabular}




\section{Study characteristics}

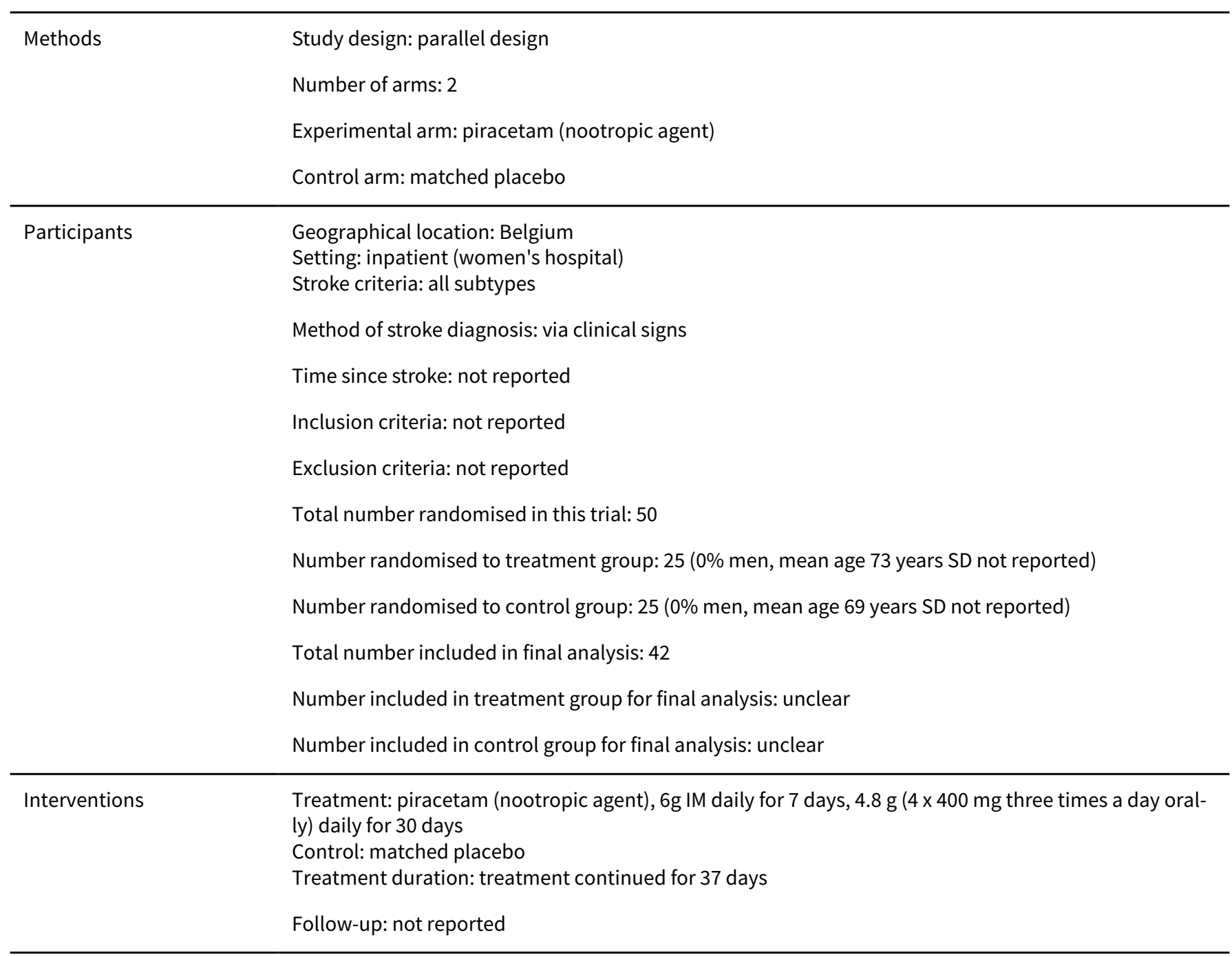

\begin{tabular}{ll}
\hline Outcomes & Primary outcomes \\
- Depression measured using the BOP scale (depressive behaviour sub scale) \\
Secondary outcomes \\
- Leaving the study early \\
- Adverse events \\
- Death
\end{tabular}

Notes

\section{Risk of bias}

\section{Bias} Authors' judgement Support for judgement

Random sequence genera- Unclear risk tion (selection bias)

Quote: "Two independent groups of patients suffering a CVA (acute stage) were randomized...." pp. 23

Comments: method of sequence generation not reported 
Creytens 1980 (Continued)

Allocation concealment Unclear risk Comments: method of allocation concealment not reported (selection bias)

Blinding of participants Unclear risk Quote: "This study was double-blind." pp. 23

and personnel (perfor-

Comments: no details of who was blinded was reported

mance bias)

All outcomes

$\begin{array}{lll}\begin{array}{l}\text { Blinding of outcome as- } \\ \text { sessment (detection bias) }\end{array} & \text { Unclear risk } & \text { Quote: "This study was double-blind." pp. } 23 \\ \text { All outcomes } & \text { Comments: no details of who was blinded was reported }\end{array}$

Incomplete outcome data High risk (attrition bias)

Comments: per protocol analysis presented only. $8 / 50$ participants were not

All outcomes included in the analysis

Selective reporting (re- Unclear risk Comments: no trial protocol available to compare with the publication porting bias)

Other bias Unclear risk

Dam 1996a

\section{Study characteristics}

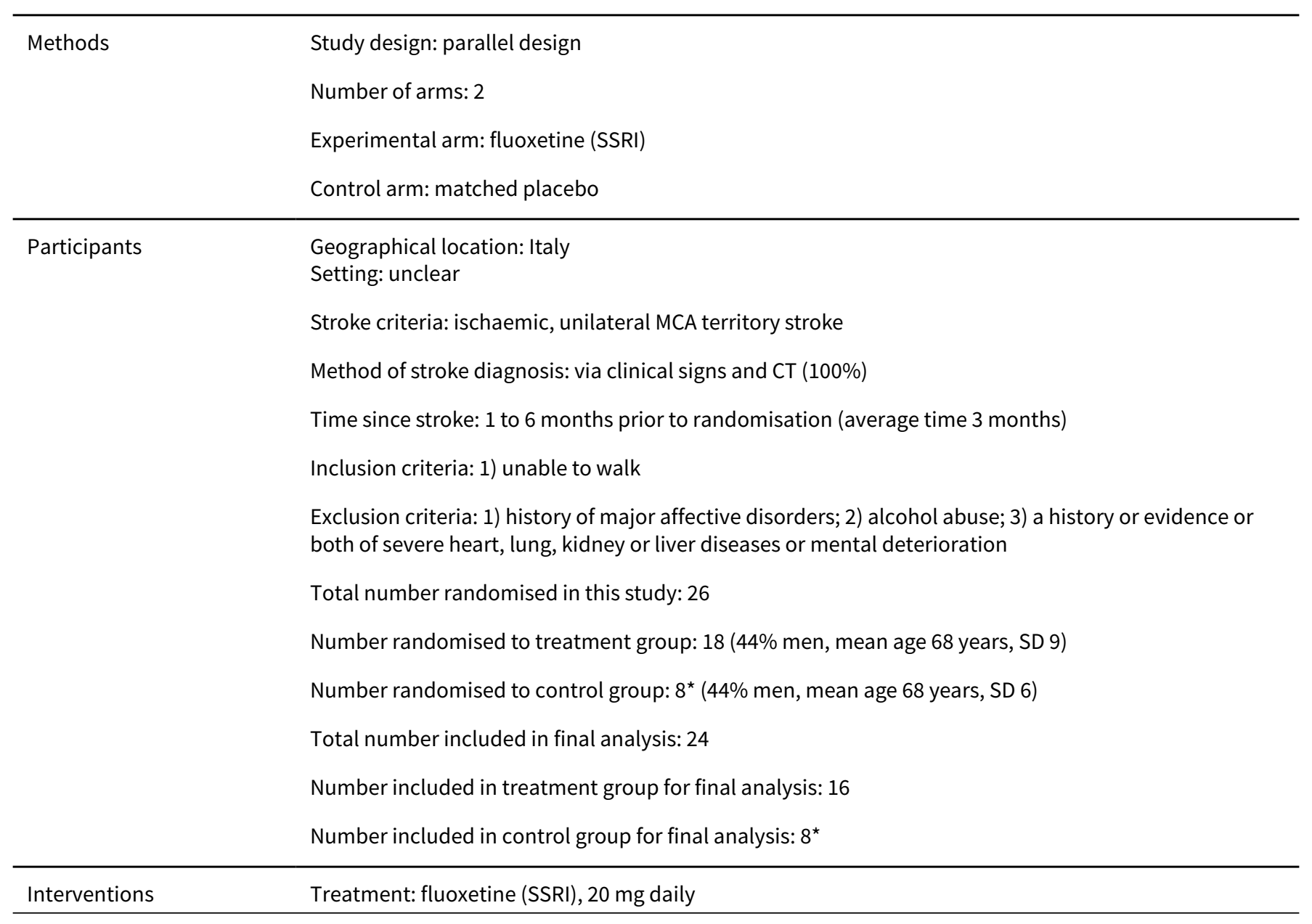

Pharmacological, psychological and non-invasive brain stimulation interventions for preventing depression after stroke (Review) 
Dam 1996a (Continued)

Control: matched placebo

Treatment duration: treatment continued on average $74+/-6$ days, duration not reported for control group

Follow-up: not reported

Primary outcomes
- Depression measured using the HDRS
Secondary outcomes
- Impairment measured using the Hemispheric Stroke Scale (HSS)
- Disability measured using the BI
- Leaving the study early
- Deathe events

Notes

\section{Risk of bias}

Bias Authors' judgement Support for judgement

Random sequence genera- Unclear risk tion (selection bias)

Quote: "Patients were randomly divided into three groups and treated for 3 months with physical therapy associated with ..." pp. 2

Comments: method of sequence generation not reported

\begin{tabular}{l}
$\begin{array}{l}\text { Allocation concealment } \\
\text { (selection bias) }\end{array}$ \\
\hline
\end{tabular}

Blinding of participants Unclear risk Comments: blinding of participants and personnel was not reported
and personnel (perfor-

mance bias)

All outcomes

\begin{tabular}{|c|c|c|}
\hline $\begin{array}{l}\text { Blinding of outcome as- } \\
\text { sessment (detection bias) }\end{array}$ & Low risk & $\begin{array}{l}\text { Quote: "The examining neurologists were blinded to the treatment adminis- } \\
\text { tered to the patients." pp. } 2\end{array}$ \\
\hline
\end{tabular}

All outcomes

\begin{tabular}{|c|c|c|}
\hline $\begin{array}{l}\text { Incomplete outcome data } \\
\text { (attrition bias) }\end{array}$ & High risk & $\begin{array}{l}\text { Comments: per protocol analyses reported only. } 3 / 35 \text { participants were not } \\
\text { included in the analysis }\end{array}$ \\
\hline
\end{tabular}

All outcomes

Selective reporting (re- Unclear risk porting bias)

Comments: no trial protocol available to compare with the publication

Other bias Low risk Comments: there was no statistically significant difference between the treatment and control group in baseline demographic characteristics

\section{Study characteristics}

Methods Study design: parallel design


Dam 1996b (Continued)

Number of arms: 2

Experimental arm: maprotiline (tetracyclic agent)

Control arm: matched placebo

\begin{tabular}{|c|c|}
\hline \multirow[t]{12}{*}{ Participants } & $\begin{array}{l}\text { Geographical location: Italy } \\
\text { Setting: unclear }\end{array}$ \\
\hline & Stroke criteria: ischaemic, unilateral MCA territory stroke. \\
\hline & Method of stroke diagnosis: via clinical signs and CT (100\%) \\
\hline & Time since stroke: 1 to 6 months prior to randomisation (average time 3 months) \\
\hline & Inclusion criteria: 1) unable to walk \\
\hline & $\begin{array}{l}\text { Exclusion criteria: } 1 \text { ) history of major affective disorders; 2) alcohol abuse; } 3 \text { ) a history or evidence or } \\
\text { both of severe heart, lung, kidney or liver diseases or mental deterioration }\end{array}$ \\
\hline & Total number randomised in this study: 26 \\
\hline & Number randomised to treatment group: 17 (43\% men, mean age 68 years, SD 8) \\
\hline & Number randomised to control group: $9^{\star}$ (44\% men, mean age 68 years, SD 6$)$ \\
\hline & Total number included in final analysis: 22 \\
\hline & Number included in treatment group for final analysis: 14 \\
\hline & Number included in control group for final analysis: $8^{\star}$ \\
\hline \multirow[t]{4}{*}{ Interventions } & Treatment: maprotiline (tetracyclic agent), 150 mg daily \\
\hline & Control: matched placebo \\
\hline & $\begin{array}{l}\text { Treatment duration: treatment continued on average } 77+/-7 \text { days, duration not reported for control } \\
\text { group }\end{array}$ \\
\hline & Follow-up: not reported \\
\hline
\end{tabular}

Primary outcomes
- Depression measured using the HDRS
Secondary outcomes
- Impairment measured using the Hemispheric Stroke Scale (HSS)
- Disability measured using the BI
- Leaving the study early
- Death

Notes

\section{Risk of bias}

\section{Bias}

Random sequence genera- Unclear risk tion (selection bias)

\section{Authors' judgement Support for judgement}

Quote: "Patients were randomly divided into three groups and treated for 3 months with physical therapy associated with ..." pp. 2

Comments: method of sequence generation not reported 


\section{Dam 1996b (Continued)}

Allocation concealment Unclear risk Comments: method of allocation not reported
(selection bias)

Blinding of participants

Unclear risk

Comments: blinding of participants and personnel was not reported and personnel (performance bias)

All outcomes

\section{Blinding of outcome as- Low risk} sessment (detection bias) All outcomes

\section{Incomplete outcome data High risk} (attrition bias)

All outcomes

\begin{tabular}{lll}
\hline $\begin{array}{l}\text { Selective reporting (re- } \\
\text { porting bias) }\end{array}$ & Unclear risk & Comments: no trial protocol available to compare with the publication \\
\hline Other bias & Low risk & $\begin{array}{l}\text { Comments: there was no statistically significant difference between the treat- } \\
\text { ment and control group in baseline demographic characteristics }\end{array}$
\end{tabular}

Forster 1996

\section{Study characteristics}

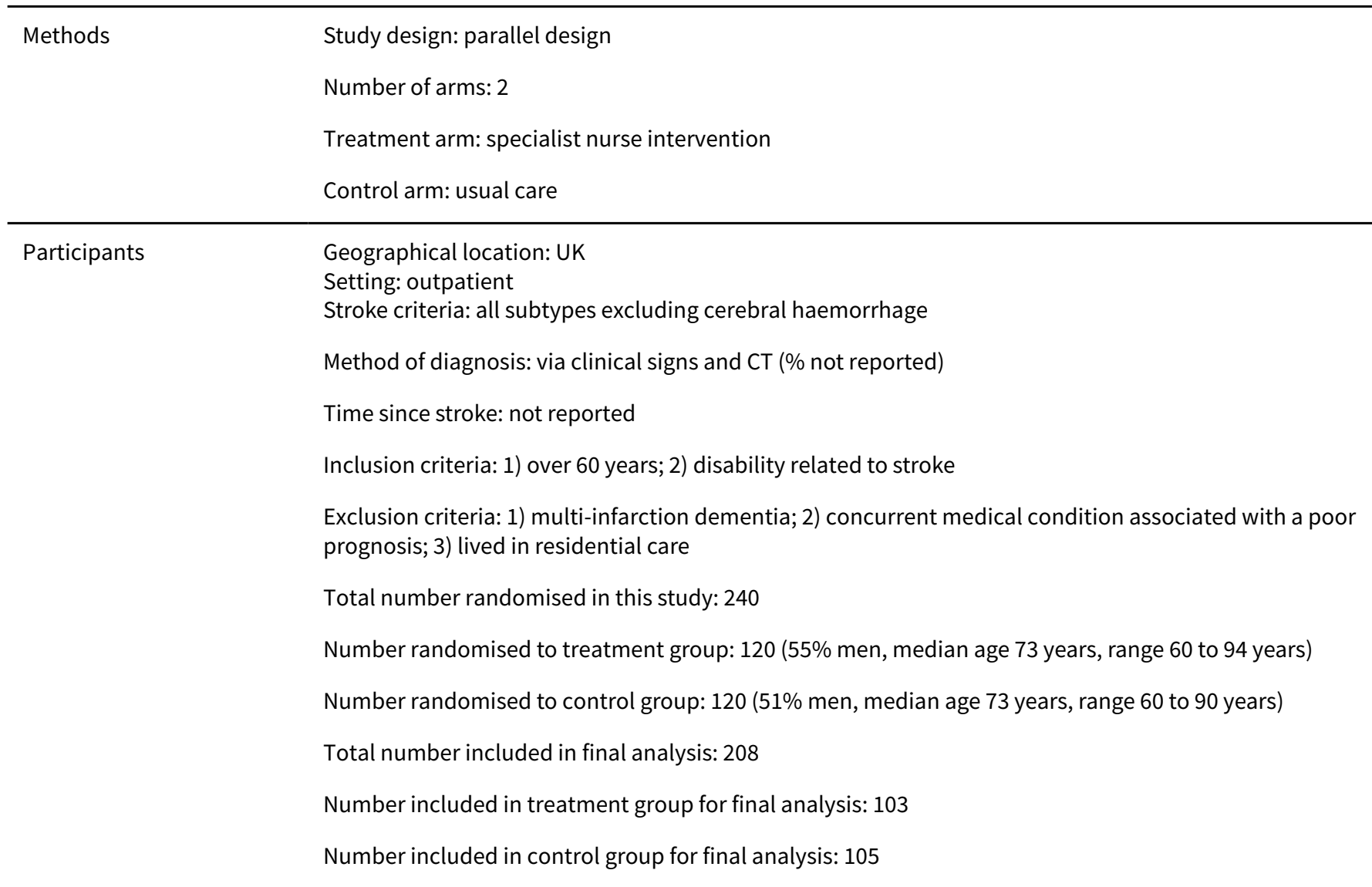


Forster 1996 (Continued)

Interventions
Treatment: specialist nurse intervention; included a counselling and enabling model, specialised in problem solving, goal setting, advice on specific issues plus information booklets

Treatment duration: treatment continued for a minimum 6 visits over the first 6 months (average of 8 visits over first 6 months)

Administed by: specialist G-grade nurses experienced in assessing disability in elderly and in problem solving approaches

Supervision: not reported

Intervention fidelity: not reported

Control: usual care, no visits

Follow-up: not reported

Primary outcomes

- Depression measured using the Nottingham Health Profile (NHP)

Secondary outcomes

- Physical function measured using the NHP,

- Disability measured using the BI

- Activities of daily living measured using the Frenchay Activities Index (FAI)

- Death

Notes

\section{Risk of bias}

\section{Bias}

Authors' judgement Support for judgement

Random sequence genera- Unclear risk tion (selection bias)

Quote: "An assistant in our department who was otherwise unconnected with the study randomized the patients by 4 length random permuted blocks of 6 to the control group or the intervention group (the patients receiving visits by the specialist nurses)." pp. 1642

Comments: method of sequence generation not reported

Allocation concealment Unclear risk Comments: method of allocation not reported
(selection bias)

Blinding of participants Unclear risk Comments: blinding of participants and personnel not reported
and personnel (perfor-

mance bias)

All outcomes

Blinding of outcome as- Unclear risk Comments: blinding of outcome assessment not reported
sessment (detection bias)

All outcomes

\section{Incomplete outcome data High risk} (attrition bias)

All outcomes
Comments: per protocol analysis reported only. 33/240 participants not included in the analysis

Selective reporting (re- Unclear risk

Comments: no trial protocol available to compare with the publication porting bias) 
Forster 1996 (Continued)

Other bias
Comments: there was no statistically significant difference between the treatment and control group in baseline demographic characteristics

Goldberg 1997

\section{Study characteristics}

\begin{tabular}{ll}
\hline Methods & Study design: parallel design \\
Number arms: 2 \\
Treatment arm: home-based therapeutic intervention \\
Control arm: usual care \\
\hline
\end{tabular}

\section{Participants}

Geographical location: USA

Setting: inpatient

Stroke criteria: unclear.

Method of stroke diagnosis: unclear.

Time since stroke: on average 2 weeks (treatment group) and 13 weeks (control group) prior to randomisation

Inclusion criteria: 1) home to return to and readily identified primary caregiver

Exclusion criteria: 1) severe pre-morbid or co-morbid conditions sufficient to impact significantly on their capacity to recover from the stroke; 2) residual cognitive or communicative impairment prevent participation in interviews

Total number randomised in this study: 55

Number randomised to treatment group: 27 (48\% men, median age 72 years, range 65 to 84 years)

Number randomised to control group: 28 ( $55 \%$ men, median age 72 years, range 65 to 81 years)

Total number included in final analysis: 41

Number included in treatment group for final analysis: 21

Number included in control group for final analysis: 20

Treatment: weekly phone contact, monthly home visits; home-based therapeutic team attend specifically to psychosocial stressors and prevent significant psychosocial problems from accelerating

Treatment duration: treatment continued for 1 year

Administered by: multidisciplinary team included a psychiatrist, psychologist, recreational therapist, research programme case manager, social worker

Supervision: case manager (social worker)

Intervention fidelity: not reported

Control: usual care, no visits

Follow-up: last follow-up at 1 year (end of treatment) 
Goldberg 1997 (Continued)

Secondary outcomes

- Leaving the study early

- Recurrent stroke

\section{Notes}

\section{Risk of bias}

\begin{tabular}{lll}
\hline Bias & Authors' judgement & Support for judgement \\
\hline $\begin{array}{ll}\text { Random sequence genera- } \\
\text { tion (selection bias) }\end{array}$ & Low risk & $\begin{array}{l}\text { Quote: "... subjects were randomized using a random number table to either } \\
\text { the experimental group, which received the STAIR program, or to a control } \\
\text { group, which did not." pp. } 67\end{array}$ \\
\hline
\end{tabular}

Allocation concealment Unclear risk
(selection bias)

\begin{tabular}{ll}
\hline $\begin{array}{l}\text { Blinding of participants } \\
\text { and personnel (perfor- }\end{array}$ & High risk \\
manote: "... since the subjects clearly could not be blinded to their own treat- \\
ment. 67
\end{tabular}
mance bias)

All outcomes

\section{Blinding of outcome as- High risk sessment (detection bias)} All outcomes

Incomplete outcome data
(attrition bias)

All outcomes

High risk

\begin{tabular}{lll}
\hline $\begin{array}{l}\text { Selective reporting (re- } \\
\text { porting bias) }\end{array}$ & Unclear risk & Comments: no trial protocol available to compare with the publication \\
\hline Other bias & Low risk & $\begin{array}{l}\text { Comments: there was no statistically significant difference between the treat- } \\
\text { ment and control group in baseline demographic characteristics }\end{array}$ \\
\hline
\end{tabular}

\section{Grade 1998}

\section{Study characteristics}

\begin{tabular}{ll}
\hline Methods & Study design: parallel design \\
& Number of arms: 2 \\
& Experimental arm: methylphenidate \\
& Control arm: matched placebo \\
\hline Participants & Geographical location: USA \\
& Stroke criteria: all subtypes \\
& Method of stroke diagnosis: via clinical signs and CT (100\%) \\
& Time since stroke: on average 18 days (SE 4, treatment group) and 19 days (SE 4, control group) prior to \\
randomisation
\end{tabular}


Grade 1998 (Continued)

\section{Inclusion criteria: not reported}

Exclusion criteria: 1) childbearing potential; 2) hypersensitivity to methylphenidate; 3) significant medical conditions, schizophrenia, delusional disorder, motor tics, uncontrolled epilepsy, malignant hypertension, prominent agitation; 4) current antidepressant treatment

Total number randomised in this study: 21

Number randomised to treatment group: 10 (40\% men, mean age 70 years, SE 4)

Number randomised to control group: 11 (64\% men, mean age 73 years, SE 3)

Total number included in final analysis: 21

Number included in treatment group for final analysis: 10

Number included in control group for final analysis: 11

Treatment: methylphenidate, $5 \mathrm{mg}$ (1 in morning) to $60 \mathrm{mg}$ ( $3 \times 10 \mathrm{mg}$ twice daily) daily; dose increased
if no adverse events reported
Control: matched placebo
Treatment duration: treatment continued for 3 to 4 weeks
Follow-up: not reported.

\begin{tabular}{ll}
\hline Outcomes & Primary outcomes \\
- Depression measured using the HDRS and ZDS \\
Secondary outcomes \\
- Dependence measured using the FIM \\
- Cognitive function measured using the MMSE \\
- Leaving the study early \\
\hline
\end{tabular}

Notes

\section{Risk of bias}

Bias Authors' judgement Support for judgement

Random sequence genera- Low risk tion (selection bias)

Allocation concealment Low risk
(selection bias)
(selection bias)

Blinding of participants Low risk
and personnel (performance bias)

All outcomes

Quote: "Using a random numbers table, the pharmacy department randomly assigned all patients to receive either methylphenidate or placebo in identical capsules." pp. 1048

\begin{tabular}{|c|c|c|}
\hline $\begin{array}{l}\text { Blinding of outcome as- } \\
\text { sessment (detection bias) } \\
\text { All outcomes }\end{array}$ & Low risk & $\begin{array}{l}\text { Quote: "To check the integrity of the double-blind design, a short question- } \\
\text { naire was given after the last rating period. Patients, examiners (psychiatrist, } \\
\text { physical therapist, and psychologist), and other study staff (nurses and physi- } \\
\text { atrist) were asked to indicate whether they believed the patient was given } \\
\text { methylphenidate or placebo (respondents could also answer 'don't know')." } \\
\text { pp. } 1048\end{array}$ \\
\hline
\end{tabular}

Quote: "... assigned all patients to receive either methylphenidate or placebo in identical capsules... " pp. 1048

Quote: "All patients and their families, as well as nurses and clinical examiners, were unaware of which participants were receiving methylphenidate." pp. 1048 
Grade 1998 (Continued)

Incomplete outcome data Low risk Comments: ITT analysis reported
(attrition bias)
All outcomes

All outcomes

Selective reporting (re- Unclear risk Comments: no trial protocol available to compare with the publication
porting bias)

\begin{tabular}{ll}
\hline Other bias $\quad$ Low risk & $\begin{array}{l}\text { Comments: there was no statistically significant difference between the treat- } \\
\text { ment and control group in baseline demographic characteristics }\end{array}$
\end{tabular}

\section{Hoffman 2015}

\section{Study characteristics}

\begin{tabular}{ll}
\hline Methods & Study design: parallel design \\
& Number of arms: 2 \\
& Experimental arm: cognitive behavioural coping skills therapy \\
Control arm: usual care & \\
Geographical location: Australia \\
Setting: inpatient \\
Stroke criteria: unclear. \\
Method of stroke diagnosis: confirmed by chart review \\
Time since stroke: not reported \\
Inclusion criteria: 1$)>18$ years old; 2$)$ adequate cognitive capacity to provide informed consent; 3) ade- \\
quate English and expressive and receptive communication skills \\
Exclusion criteria: 1$)$ neurodegenerative disorder e.g. dementia; 2 ) living > 50 km away from hospital \\
Depression criteria: depression score not an entry criteria. For unpublished analysis \\
HADS $\geq 8$ used for depression criteria \\
Total number randomised in this trial: 16 \\
Number randomised to treatment group: 11 (63.6\% men, mean age 63.6, SD 13.0) \\
Number randomised to control group: 5 (60\% men, mean age 57.0, SD 14.2) \\
Total number included in final analysis: 14 \\
Number included in treatment group for final analysis: 9 \\
Number included in control group for final analysis: 5
\end{tabular}

Interventions

Treatment: $8 \times 1$-hour cognitive-behavioural coping skills sessions delivered by a clinical psychologist with the first 2 in hospital and 6 delivered at home. The sessions included cognitive and behavioural exercises to prepare individuals for discharge and to adjust post-discharge (e.g. psychoeducation, selfmonitoring, graduated activity participation, and cognitive restructuring)

Treatment duration: 8 weeks

Administered by: clinical psychologist

Supervision: clinical psychologist

Intervention fidelity: 10/11 (90\%) in the treatment group (1 discontinued after 1 session) completed the sessions and 5/5 (100\%) in the control group

Control: usual care

Follow-up: 3 months

\section{Outcomes}

Primary outcomes

- Depression measured using the MADRS and the HADS 
Hoffman 2015 (Continued)

- Anxiety measured using the MADRS and the HADS

Secondary outcomes

- Activities of daily living measured using the MBI

- Stress coping measured using the Stress Appraisal Coping Measure

Notes

This trial had 3 arms (self-management therapy, cognitive behavioural therapy and usual care) but only the data from the cognitive behavioural therapy compared with usual care ( $n=14$ participants) are presented here

\section{Risk of bias}

Bias Authors' judgement Support for judgement

\begin{tabular}{ll}
\hline $\begin{array}{l}\text { Random sequence genera- } \\
\text { tion (selection bias) }\end{array}$ & Low risk \\
\end{tabular}
tion (selection bias) randomization sequence ..." pp. 118

\begin{tabular}{lll}
\hline $\begin{array}{l}\text { Allocation concealment } \\
\text { (selection bias) }\end{array}$ & Unclear risk & Comments: method of allocation concealment was not reported \\
\hline $\begin{array}{l}\text { Blinding of participants } \\
\text { and personnel (perfor- } \\
\text { mance bias) }\end{array}$ & High risk & $\begin{array}{l}\text { Comments: due to the nature of the intervention blinding will not be achiev- } \\
\text { able for participants or staff delivering care }\end{array}$ \\
\hline $\begin{array}{l}\text { Blinding of outcome as- } \\
\text { sessment (detection bias) }\end{array}$ & Low risk & $\begin{array}{l}\text { Quote: "Outcomes were assessed in a face-to-face interview conducted by a } \\
\text { research assistant (a registered psychologist) who was blind to group alloca- } \\
\text { tion." pp. } 118\end{array}$ \\
\hline $\begin{array}{l}\text { Incomplete outcome data } \\
\text { (attrition bias) } \\
\text { All outcomes }\end{array}$ & Low risk & $\begin{array}{l}\text { Quote: "Analysis was completed using ... and on an intention to treat basis and } \\
\text { missing data were addressed using the last observation carried forward proce- } \\
\text { dure." pp. } 120\end{array}$
\end{tabular}

\begin{tabular}{lll}
\hline $\begin{array}{l}\text { Selective reporting (re- } \\
\text { porting bias) }\end{array}$ & Unclear risk & Comments: no trial protocol available to compare with the publication \\
\hline Other bias & Low risk & $\begin{array}{l}\text { Comments: there was no statistically significant difference between the treat- } \\
\text { ment and control group in baseline demographic characteristics }\end{array}$ \\
\hline
\end{tabular}

House 2000

\section{Study characteristics}

\begin{tabular}{ll}
\hline Methods & Study design: parallel design \\
& Number of arms: 3 \\
& Experimental arm: problem-solving therapy \\
& Control arm 1: non-specific volunteer visits \\
& Control arm 2: usual care \\
\hline Participants & Geographical location: UK \\
Setting: outpatient \\
Stroke criteria: ischaemic stroke and primary intracerebral haemorrhage
\end{tabular}


Method of stroke diagnosis: via clinical signs and CT (\% not reported)

Time since stroke: 0 to 1 month prior to randomisation

Inclusion criteria: 1) local resident

Exclusion criteria: too ill to interview, impaired speech, cognitive impairment or poor English, living in a residential home at admission, serious concurrent illness likely to dominate pattern of care, participation in another rehabilitation trial

Total number randomised in this study: 450

Number randomised to treatment group: 151 (56\% men, median age 74 years, IQR 65 to 79 )

Number randomised to control group 1: $149^{\wedge}$ (49\% men, median age 72 years, IQR 64 to 78 )

Number randomised to control group 2: $150^{\wedge}$ ( $57 \%$ men, median age 74 years, IQR 66 to 80 )

Total number included in final analysis: 450

Number included in treatment group for final analysis: 151

Number included in control group 1 for final analysis: 149

Number included in control group 2 for final analysis: 150

Interventions

Treatment: problem-solving therapy (manual-based): sessions in participants' home, help to identify and prioritise problems, set goals and identify solutions to problems

Treatment duration: treatment continued for 1 to 10 (median 5) fortnightly sessions for treatment group, 1 to 42 (median 6) fortnightly contacts for attention control group

Administered by: problem-solving therapy delivered by psychiatric nurses, attention control administered by trained volunteers

Supervision: specialised liaison psychiatrist

Intervention fidelity: $\mathrm{n}=121$ participants received standard problem solving intervention, $\mathrm{n}=30$ did not receive full intervention

Control: 1) attention control -non-specific volunteer visits, 2) Usual care, no visits

Follow-up: not reported

Primary outcomes
- Depression (difference between groups at end of treatment) measured using the GHQ-28 total score ${ }^{\star \star}$
- Depression (proportion scoring $>4$ ) measured using the Present State Examination (PSE)
Secondary outcomes
- Leaving the study early
- Death

Notes

\section{Risk of bias}

\section{Bias}

Authors' judgement Support for judgement

Random sequence genera- Low risk tion (selection bias)
Quote: "Random allocation was generated by computer in the trials office in blocks of 15 , stratified by admitting NHS trusts." pp. 2 
House 2000 (Continued)

Allocation concealment Low risk Quote: "Patients were sent a letter confirming their participation and giv(selection bias) ing the name of their volunteer or nurse, as appropriate, and the planned follow-up dates. In this way, patients were not aware that their treatment was being randomly allocated, and did not know that other patients were receiving a different treatment." pp. 2
Blinding of participants High risk and personnel (performance bias)

All outcomes
Quote: "Patients were not masked to their allocation as this is clearly impossible to achieve with this type of intervention. However, as a result of the randomization procedure patients were unaware of other treatment allocations." pp. 2

Comments: blinding of personnel not reported.
Blinding of outcome as- High risk sessment (detection bias) All outcomes
Quote: "Follow up assessments took place in the patient's home with an interviewer who was not informed of the treatment allocation of the patient. Patients who received problem-solving were asked by the therapist not to reveal their allocation. To test the extent of unmasking of outcome assessors, we asked the interviewer to guess the allocation of 127 patients seen at 12 months." pp. 3

"The interviewer guessed correctly in 65 (51.2\%) patients (kappa $=0.26, \mathrm{p}<$ 0.001 ), suggesting that some unmasking had occurred." pp. 4

Comments: ITT analysis reported. All participants were included in the analy-

$\begin{array}{ll}\text { Incomplete outcome data } & \text { Low risk } \\ \text { (attrition bias) } & \text { sis }\end{array}$

All outcomes

Selective reporting (re- Unclear risk Comments: no trial protocol available to compare with the publication
porting bias)

Other bias Low risk
Comments: there was no statistically significant difference between the treatment and control group in baseline demographic characteristics

\section{Kerr 2018}

\section{Study characteristics}

\begin{tabular}{|c|c|}
\hline Methods & $\begin{array}{l}\text { Study design: parallel design } \\
\text { Number of arms: } 2 \\
\text { Experimental arm: individual motivational interviewing } \\
\text { Control arm: usual care }\end{array}$ \\
\hline Participants & $\begin{array}{l}\text { Geographical location: Australia } \\
\text { Setting: inpatient } \\
\text { Stroke criteria: acute presentation after acute stroke (cerebral infarction/intracerebral haemorrhage) } \\
\text { Method of stroke diagnosis: confirmed by neurologist in the medical notes } \\
\text { Time since stroke: not reported } \\
\text { Inclusion criteria: } 1 \text { ) acute presentation after acute stroke (cerebral infarction/intracerebral haemor- } \\
\text { rhage; 2) cognitively alert } \\
\text { Exclusion criteria: } 1 \text { ) mental health conditions, including depressive symptoms requiring profession- } \\
\text { al support within } 1 \text { month; } 2 \text { ) severe communication problems e.g. significant dysphasia or aphasia; } 3 \text { ) } \\
\text { myocardial infarction; } 4) \text { concurrent neurological disease/trauma; 5) subarachnoid haemorrhage } \\
\text { Depression criteria: depression score not an entry criteria. } \\
\text { Total number randomised in this trial: } 28 \\
\text { Number randomised to treatment group: } 14 \text { ( } 85.7 \% \text { men, mean age } 69.14, \text { SD 10.91) } \\
\text { Number randomised to control group: } 14 \text { ( } 50 \% \text { men, mean age } 71.95, \text { SD 13.14) }\end{array}$ \\
\hline
\end{tabular}

Pharmacological, psychological and non-invasive brain stimulation interventions for preventing depression after stroke (Review) 
Kerr 2018 (Continued)

Total number included in final analysis: 25

Number included in treatment group for final analysis: 12

Number included in control group for final analysis: 13

Interventions

Treatment: $3 \times 30$-minute sessions of motivational interviewing. The purpose of Session 1 was to set the agenda and encourage the patient to talk about adjustment to stroke. In Session 2, the patient was encouraged to identify realistic goals for recovery and identify barriers to achieving goals. In Session 3, the goal was to identify any ambivalence the patient had about achieving goals; support the patient's optimism and self-efficacy, and assist identification of solutions to solve problems. Participants were encouraged to summarise their goals and commitment and clarify any information from the first 2 sessions

Treatment duration: 3 months

Administered by: trained facilitators

Supervision: by a psychologist

Intervention fidelity: unclear

Control: usual care

Follow-up: 3 months

Primary outcomes
- Depression measured using the HADS and the PHQ-9
- Anxiety measured using the HADS
Secondary outcomes
- Quality of life measured using the QoL Index

Notes

\section{Risk of bias}

\begin{tabular}{lll}
\hline Bias & Authors' judgement & Support for judgement \\
\hline $\begin{array}{l}\text { Random sequence genera- } \\
\text { tion (selection bias) }\end{array}$ & Low risk & $\begin{array}{l}\text { Quote: "A computer-generated block randomization list equally divided all } \\
\text { numbers between } 1 \text { and } 60 \text { into either treatment or control groups." pp. } 3\end{array}$ \\
\hline $\begin{array}{l}\text { Allocation concealment } \\
\text { (selection bias) }\end{array}$ & High risk & $\begin{array}{l}\text { Quote: "Allocation to the intervention or control arms was concealed from } \\
\text { participants until after recruitment and baseline data collection. Envelopes } \\
\text { were prepared by the Principal Investigator and stored in a locked cupboard } \\
\text { in the ward. The envelopes were numbered sequentially, indicating the order } \\
\text { in which participants were enrolled into the study (e.g. the first participant re- } \\
\text { ceived the envelope labelled 'Number 1', the second participant received the } \\
\text { envelope 'Number 2', etc.). A note in the envelope indicated the allocation (to } \\
\text { intervention or control group), concealed by coloured paper to protect the } \\
\text { identity of the allocation group. The project manager opened the randomiza- } \\
\text { tion envelopes after baseline data collection." pp. } 3\end{array}$
\end{tabular}

\begin{tabular}{lll}
\hline $\begin{array}{l}\text { Blinding of participants } \\
\text { and personnel (perfor- }\end{array}$ & High risk & $\begin{array}{l}\text { Quote: "Allocation to the intervention or control arms was concealed from } \\
\text { participants until after recruitment and baseline data collection." pp. } 3\end{array}$
\end{tabular}
mance bias)

All outcomes

Comments: blinding of personnel not reported

Blinding of outcome as-
sessment (detection bias)
sessment (detection bias)

Quote: "The research assistant, a nurse with significant research experience, All outcomes was employed to collect data at the 2 follow-up time points. Although inten- 
Kerr 2018 (Continued)

tionally blinded, the research assistant may have become aware of the allocation in conversation with the participant." pp. 5

\begin{tabular}{lll}
\hline Incomplete outcome data & High risk & Comments: per protocol analysis reported in Table 1 and 2. 10/48 participants \\
(attrition bias) & were not included in the analysis. (8 dropped out; 2 unable to participate; 1 \\
All outcomes & died, 1 developed aphasia)
\end{tabular}

Selective reporting (re- Unclear risk Comments: no trial protocol available to compare with the publication
porting bias)

Other bias Low risk

Quote: "Patients were broadly similar for age, gender, nationality, and marital status. Capacity to perform activities of daily living were similar, according to the Modified Barthel Index." pp. 6

Comments: no other bias detected

\section{Palomaki 1999}

\section{Study characteristics}

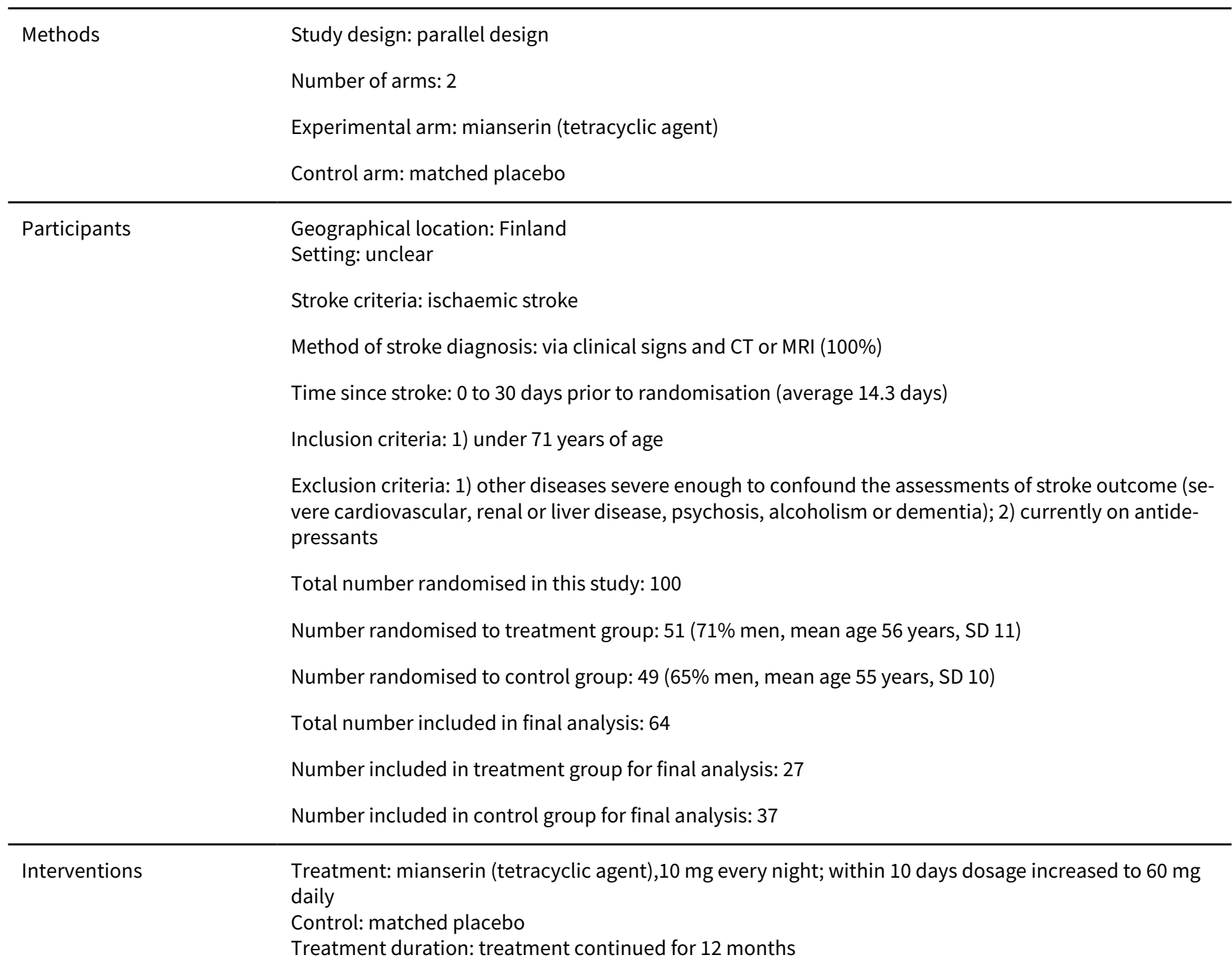


Palomaki 1999 (Continued)

Follow-up: 18 months

Primary outcomes
- Depression (proportion not meeting criteria for major depression) measured using the DSM-III-R
- Depression (change in scores from baseline to end of treatment) measured using the HDRS and the BDI
Secondary outcomes
- Adverse events

Notes

\section{Risk of bias}

\begin{tabular}{|c|c|c|}
\hline Bias & Authors' judgement & Support for judgement \\
\hline $\begin{array}{l}\text { Random sequence genera- } \\
\text { tion (selection bias) }\end{array}$ & Unclear risk & $\begin{array}{l}\text { Quote: "The patients were randomized to mianserin or placebo groups of } \\
\text { equal size, stratified according to location of lesion ..." pp. } 490 \\
\text { Comments: method of sequence generation not reported }\end{array}$ \\
\hline $\begin{array}{l}\text { Allocation concealment } \\
\text { (selection bias) }\end{array}$ & High risk & $\begin{array}{l}\text { Quote: "The randomization codes were kept in sealed envelopes." pp. } 491 \\
\text { Comments: this method of concealment can be tampered with }\end{array}$ \\
\hline $\begin{array}{l}\text { Blinding of participants } \\
\text { and personnel (perfor- } \\
\text { mance bias) } \\
\text { All outcomes }\end{array}$ & Low risk & $\begin{array}{l}\text { Quote: "For each stratum, the test drugs were provided in numbered vials con- } \\
\text { taining tablets of identical appearance." pp. } 491\end{array}$ \\
\hline
\end{tabular}

\begin{tabular}{lll}
\hline $\begin{array}{l}\text { Blinding of outcome as- } \\
\text { sessment (detection bias) } \\
\text { All outcomes }\end{array}$ & Unclear risk & Comments: blinding of outcome assessment not reported \\
\hline $\begin{array}{l}\text { Incomplete outcome data } \\
\text { (attrition bias) }\end{array}$ & High risk & $\begin{array}{l}\text { Comments: per protocol analysis reported only. Only } 75 \% \text { of participants re- } \\
\text { mained in placebo and } 52 \% \text { remained in treatment group at } 12 \text { months and } \\
\text { were included in the analysis }\end{array}$ \\
\hline
\end{tabular}

\begin{tabular}{lll}
\hline $\begin{array}{l}\text { Selective reporting (re- } \\
\text { porting bias) }\end{array}$ & Unclear risk & Comments: no trial protocol available to compare with the publication \\
\hline Other bias & Unclear risk & Comments: the treatment group had more heart disease \\
\hline
\end{tabular}

Rasmussen 2003

\section{Study characteristics}

\begin{tabular}{ll}
\hline Methods & Study design: parallel design \\
& Number of arms: 2 \\
& Experimental arm: sertraline (SSRI) \\
& Control arm: matched placebo \\
\hline Participants & Geographical location: Denmark \\
& Setting: unclear
\end{tabular}


Stroke criteria: ischaemic and primary intracerebral haemorrhage

Method of stroke diagnosis: via clinical signs and symptoms

Time since stroke: 0 to 4 weeks prior to randomisation

Inclusion criteria: not reported

Exclusion criteria: 1) current depression; 2) significant aphasia or dementia; 3) history of schizophrenia; 4) psychosis or severe drug abuse; 5) pre-existing neurological illness; 6) antidepressants in preceding 4 weeks; 7) current (within 6 months) cardiovascular illness

Total number randomised in this study: 137

Number randomised to treatment group: 70 (50\% men, mean age 72 years, SD 9)

Number randomised to control group: 67 (51\% men, mean age 68 years, SD 11)

Total number included in final analysis: 118

Number included in treatment group for final analysis: 61

Number included in control group for final analysis: 57

Interventions

Treatment: sertraline (SSRI), $50 \mathrm{mg}$ daily; at any time after 2 weeks dose could be increased in $50 \mathrm{mg}$ increments up to $150 \mathrm{mg}$ daily; average dose $62.9 \mathrm{mg}$ daily

Control: matched placebo

Treatment duration: treatment continued for 12 months

Follow-up: not reported

Primary outcomes
- Depression (change in scores from baseline to end of treatment) measured using the HDRS
- Depression (proportion scoring $>2$ ) measured using the CGI
- Depression (proportion scoring $>16$ ) measured using the GDS
Secondary outcomes
- Leaving the study early

Notes

\section{Risk of bias}

Bias Authors' judgement Support for judgement

\begin{tabular}{lll}
\hline Random sequence genera- & Unclear risk & Quote: "Patients were randomly assigned to 12 months of double-blind study \\
tion (selection bias) & treatment." pp. 217
\end{tabular}
tion (selection bias) treatment." pp. 217

Comments: method of sequence generation not reported

\begin{tabular}{|c|c|c|}
\hline $\begin{array}{l}\text { Allocation concealment } \\
\text { (selection bias) }\end{array}$ & Unclear risk & Comments: method of allocation concealment not reported \\
\hline $\begin{array}{l}\text { Blinding of participants } \\
\text { and personnel (perfor- } \\
\text { mance bias) }\end{array}$ & Low risk & $\begin{array}{l}\text { Quote: title - "A double-blind, placebo-controlled study of sertraline in the pre- } \\
\text { vention of depression in stroke patients." pp. } 216\end{array}$ \\
\hline All outcomes & & Comments: who was blinded was not reported \\
\hline $\begin{array}{l}\text { Blinding of outcome as- } \\
\text { sessment (detection bias) }\end{array}$ & Low risk & $\begin{array}{l}\text { Quote: title - "A double-blind, placebo-controlled study of sertraline in the pre- } \\
\text { vention of depression in stroke patients." pp. } 216\end{array}$ \\
\hline
\end{tabular}


Rasmussen 2003 (Continued)

All outcomes
Comments: who was blinded was not reported
Incomplete outcome data High risk (attrition bias)

All outcomes
Comments: per protocol analysis reported only. 19/137 were not included in the analysis

\section{Selective reporting (reporting bias)

Other bias Unclear risk Comments: the treatment group were older on average

\section{Study characteristics}

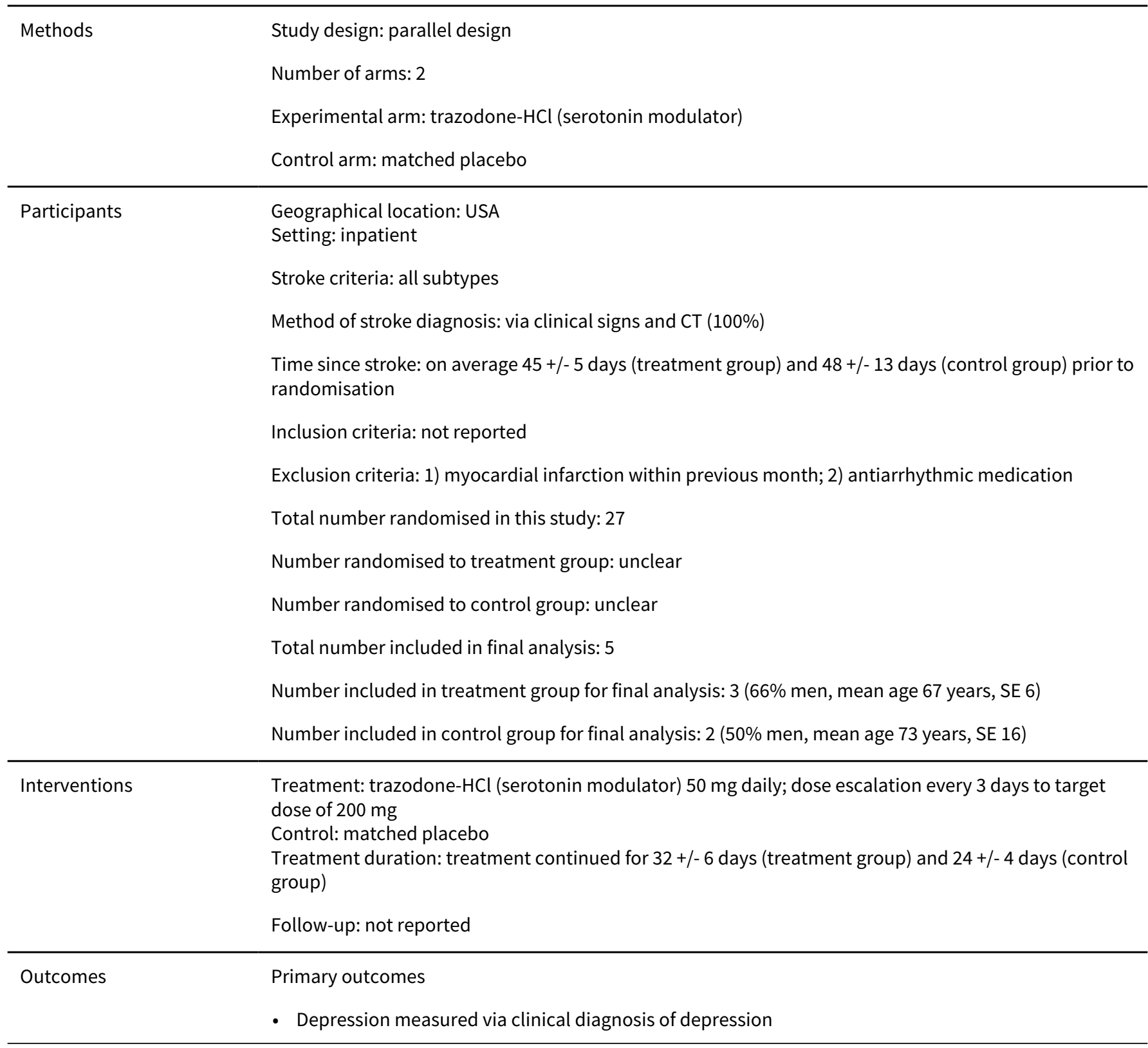

Pharmacological, psychological and non-invasive brain stimulation interventions for preventing depression after stroke (Review) 54 Copyright $\odot 2020$ The Cochrane Collaboration. Published by John Wiley \& Sons, Ltd. 
Reding 1986 (Continued)

\title{
Secondary outcomes
}

- Activities of daily living measured using the $\mathrm{BI}$

\section{Notes}

\section{Risk of bias}

\begin{tabular}{|c|c|c|}
\hline Bias & Authors' judgement & Support for judgement \\
\hline $\begin{array}{l}\text { Random sequence genera- } \\
\text { tion (selection bias) }\end{array}$ & Low risk & $\begin{array}{l}\text { Quote: "Patients were assigned to either treatment or placebo groups accord- } \\
\text { ing to a table of random numbers." pp. } 763\end{array}$ \\
\hline $\begin{array}{l}\text { Allocation concealment } \\
\text { (selection bias) }\end{array}$ & Unclear risk & Comments: method of allocation concealment not reported \\
\hline $\begin{array}{l}\text { Blinding of participants } \\
\text { and personnel (perfor- } \\
\text { mance bias) } \\
\text { All outcomes }\end{array}$ & Low risk & $\begin{array}{l}\text { Quote: "Trazodone hydrochloride ( } 50 \mathrm{mg} \text { ) or placebo in an identical capsule } \\
\text { was administered orally by the nursing staff ..." pp. } 763\end{array}$ \\
\hline $\begin{array}{l}\text { Blinding of outcome as- } \\
\text { sessment (detection bias) } \\
\text { All outcomes }\end{array}$ & Low risk & $\begin{array}{l}\text { Quote: "... attending physician, unaware of treatment group assignment, not- } \\
\text { ed signs or symptoms possibly due to trazodone ..." pp. } 764\end{array}$ \\
\hline $\begin{array}{l}\text { Incomplete outcome data } \\
\text { (attrition bias) } \\
\text { All outcomes }\end{array}$ & High risk & $\begin{array}{l}\text { Comments: only } 5 / 27 \text { patients completed the study at target dose. The study } \\
\text { was discontinued due to perceived side effects in both groups. The analysis } \\
\text { is presented in subgroups with only numbers of improved not total numbers } \\
\text { analysed }\end{array}$ \\
\hline $\begin{array}{l}\text { Selective reporting (re- } \\
\text { porting bias) }\end{array}$ & Unclear risk & Comments: no trial protocol available to compare with the publication \\
\hline Other bias & Low risk & $\begin{array}{l}\text { Comments: there was no statistically significant difference between the treat- } \\
\text { ment and control group in baseline demographic characteristics }\end{array}$ \\
\hline
\end{tabular}

Robinson 2000a

\section{Study characteristics}

\begin{tabular}{ll}
\hline Methods & Study design: parallel design \\
Number of arms: 2 \\
Experimental arm: fluoxetine (SSRI) \\
Control arm: matched placebo
\end{tabular}

\section{Participants}

\author{
Geographical location: USA and Argentina \\ Setting: mixed \\ Stroke criteria: all subtypes \\ Method of stroke diagnosis: via clinical signs and CT (100\%) \\ Time since stroke: on average 8 weeks (treatment) and 5 weeks (control) prior to randomisation \\ Inclusion criteria: 1) stroke within 6 months of recruitment; 2) 18 to 85 years of age
}


Robinson 2000a (Continued)

Exclusion criteria: 1) other significant medical illness; 2) severe comprehension deficit; 3) prior history of head injury; 4) prior history of other brain disease (with the exception of stroke); 5) participants on antidepressants (other than fluoxetine) were allowed to stop their antidepressants for a 2-week washout period

Total number randomised in this study: 25

Number randomised to treatment group: 17 ( $88 \%$ men, mean age 66 years, SD 13)

Number randomised to control group: $8^{\star}$ ( $75 \%$ men, mean age 67 years, SD 9)

Total number included in final analysis: unclear

Number included in treatment group for final analysis: unclear

Number included in control group for final analysis: unclear

Interventions

Treatment: fluoxetine (SSRI),10 mg daily (3 weeks), $20 \mathrm{mg}$ daily (3 weeks), $30 \mathrm{mg}$ daily (3 weeks), $40 \mathrm{mg}$ daily (3 weeks)

Control: matched placebo

Treatment duration: treatment continued for 12 weeks

Follow-up: none

Primary outcomes
- Depression measured using the HDRS
Secondary outcomes
- Cognitive function measured using the MMSE
- Activities of daily living measured using the Johns Hopkins Functioning Inventory
- Adverse events
- Death

Notes

\section{Risk of bias}

Bias Authors' judgement Support for judgement

Random sequence genera- Unclear risk tion (selection bias)
Quote: "All patients were randomly assigned to either active or placebo medication." pp. 352

Comments: method of sequence generation not reported.

\begin{tabular}{lll}
\hline $\begin{array}{l}\text { Allocation concealment } \\
\text { (selection bias) }\end{array}$ & Unclear risk & Comments: method of allocation concealment not reported. \\
\hline $\begin{array}{l}\text { Blinding of participants } \\
\text { and personnel (perfor- } \\
\text { mance bias) }\end{array}$ & Low risk & $\begin{array}{l}\text { Quote: "To maintain the blind, doses were decreased for equal numbers of } \\
\text { placebo patients. The active and placebo pills were identical." pp. } 353\end{array}$ \\
All outcomes & &
\end{tabular}

\begin{tabular}{lll}
\hline $\begin{array}{l}\text { Blinding of outcome as- } \\
\text { sessment (detection bias) } \\
\text { All outcomes }\end{array}$ & Low risk & $\begin{array}{l}\text { Comments: blinding of outcome assessment not reported. However, the } \\
\text { study authors state that this trial is 'double-blind' }\end{array}$ \\
\hline $\begin{array}{l}\text { Incomplete outcome data } \\
\text { (attrition bias) } \\
\text { All outcomes }\end{array}$ & High risk & $\begin{array}{l}\text { Comments: Table } 1 \text { presents ITT analysis but per protocol analyses reported. } \\
\text { The number excluded from analysis varies }\end{array}$ \\
\hline \hline
\end{tabular}


Robinson 2000a (Continued)

Selective reporting (re- Unclear risk Comments: no trial protocol available to compare with the publication porting bias)

Other bias

Low risk

Comments: there was no statistically significant difference between the treatment and control group in baseline demographic characteristics

Robinson 2000b

\section{Study characteristics}

\begin{tabular}{ll}
\hline Methods & Study design: parallel design \\
& Number of arms: 2 \\
Experimental arm: nortriptyline (tricyclic agent) \\
Control arm: matched placebo
\end{tabular}

\section{Participants}

Geographical location: USA and Argentina

Setting: mixed

Stroke criteria: all subtypes

Method of stroke diagnosis: via clinical signs and CT (100\%);

Time since stroke: on average 10 weeks (treatment) and 5 weeks (control) prior to randomisation

Inclusion criteria: 1) stroke within 6 months of recruitment; 2) 18 to 85 years of age

Exclusion criteria: 1) other significant medical illness; 2) severe comprehension deficit; 3) prior history of head injury; 4) prior history of other brain disease (with the exception of stroke); 5) participants on antidepressants (other than fluoxetine) were allowed to stop their antidepressants for a 2-week washout period

Total number randomised in this study: 23

Number randomised to treatment group: 15 (47\% men, mean age 65 years, SD 13)

Number randomised to control group: $8^{\star}$ (75\% men, mean age 67 years, SD 9)

Total number included in final analysis: unclear

Number included in treatment group for final analysis: unclear

Number included in control group for final analysis: unclear

Interventions $\quad$ Treatment: nortriptyline (tricyclic agent), $25 \mathrm{mg}$ daily (1 week), $50 \mathrm{mg}$ daily ( 2 weeks), $75 \mathrm{mg}$ daily (4 weeks), $100 \mathrm{mg}$ daily (6 weeks)

Control: matched placebo

Treatment duration: treatment continued for 12 weeks

Follow-up: not reported

Outcomes

Primary outcomes

- Depression measured using the HDRS

Secondary outcomes

- Cognitive function measured using the MMSE

- Activities of daily living measured using the Johns Hopkins Functioning Inventory 
Robinson 2000b (Continued)

$$
\begin{aligned}
& \text { - Adverse events } \\
& \text { - Death }
\end{aligned}
$$

\section{Notes}

\section{Risk of bias}

\begin{tabular}{lll}
\hline Bias & Authors' judgement & Support for judgement \\
\hline $\begin{array}{l}\text { Random sequence genera- } \\
\text { tion (selection bias) }\end{array}$ & Unclear risk & $\begin{array}{l}\text { Quote: "All patients were randomly assigned to either active or placebo med- } \\
\text { ication." pp. } 352\end{array}$
\end{tabular}

Comments: method of sequence generation not reported

\begin{tabular}{|c|c|c|}
\hline $\begin{array}{l}\text { Blinding of participants } \\
\text { and personnel (perfor- }\end{array}$ & Low risk & $\begin{array}{l}\text { Quote: "To maintain the blind, doses were decreased for equal numbers of } \\
\text { placebo patients. The active and placebo pills were identical." pp. } 353\end{array}$ \\
\hline
\end{tabular}

Allocation concealment $\quad$ Unclear risk
(selection bias)

mance bias)

All outcomes

$\begin{array}{ll}\begin{array}{l}\text { Blinding of outcome as- } \\ \text { sessment (detection bias) }\end{array} \quad \text { Low risk } & \begin{array}{l}\text { Comments: blinding of outcome assessment not reported. However, the } \\ \text { study authors state that this trial is 'double-blind' }\end{array}\end{array}$

\begin{tabular}{|c|c|c|}
\hline $\begin{array}{l}\text { Incomplete outcome data } \\
\text { (attrition bias) }\end{array}$ & High risk & $\begin{array}{l}\text { Comments: Table } 1 \text { presents ITT analysis but per protocol analyses reported. } \\
\text { The number excluded from analysis varies }\end{array}$ \\
\hline
\end{tabular}

All outcomes

All outcomes

The number excluded from analysis varies

\begin{tabular}{lll}
\hline $\begin{array}{l}\text { Selective reporting (re- } \\
\text { porting bias) }\end{array}$ & Unclear risk & Comments: no trial protocol available to compare with the publication \\
\hline Other bias & Low risk & $\begin{array}{l}\text { Comments: there was no statistically significant difference between the treat- } \\
\text { ment and control group in baseline demographic characteristics }\end{array}$ \\
\hline
\end{tabular}

Robinson 2008

\section{Study characteristics}

\begin{tabular}{ll}
\hline Methods & Study design: parallel design \\
& Number of arms: 2 \\
Experimental arm: escitalopram (SSRI) & Control arm: matched placebo \\
\hline Participants & Geographical location: USA \\
& Setting: outpatient \\
Stroke criteria: ischaemic or cerebellar stroke & \\
& Method of stroke diagnosis: based on clinical and neuro-radiological findings consistent with either \\
& hemispheric, brainstem or cerebellar stroke \\
& Time since stroke: not reported \\
Inclusion criteria: 1 ) patients with either ischaemic or cerebellar stroke \\
Exclusion criteria: 1 ) if they met DSM-IV diagnostic criteria for major or minor (research criteria) depres- \\
sive disorder; 2 ) those with severe comprehension deficit as demonstrated by inability to complete part \\
1 of the Token Test or patients with neuropsychological testing who showed impaired decision-mak-
\end{tabular}


Robinson 2008 (Continued)

ing capacity; 3) patients with acute coronary syndromes; 4) those with stroke as secondary complications from an intracranial aneurysm, arterial-venous malformation, stroke from a myocardial infarction, aortic dissection or revascularization therapy; 5) life-threatening heart or respiratory failure, renal or hepatic failure, severely disabling musculoskeletal disorder, cancer and neurodegenerative disorders such as idiopathic Parkinson disease or Alzheimer disease; 6) those who met the DSM-IV criteria for alcohol or substance abuse or dependence within the past 12 months

Depression criteria: depression score not an entry criteria

Total number randomised in this trial: 117

Number randomised to treatment group: 59 (64.4\% men, mean age 61.3, SD 13.7)

Number randomised to control group: 58 (63.8\% men, mean age 63.9, SD 13.3)

Total number included in final analysis: 117

Number included in treatment group for final analysis: 59

Number included in control group for final analysis: 58

\begin{tabular}{|c|c|}
\hline Interventions & $\begin{array}{l}\text { Treatment: escitalopram (SSRI) } 10 \mathrm{mg} / \text { day (morning) for participants }<65 \text { years and } 5 \mathrm{mg} / \text { day for par- } \\
\text { ticipants }>65 \text { years } \\
\text { Control: matched placebo } \\
\text { Treatment duration: } 12 \text { months } \\
\text { Follow-up: not reported }\end{array}$ \\
\hline Outcomes & $\begin{array}{l}\text { Primary outcomes } \\
\text { - Depression measured using the } 17 \text {-item HDRS } \\
\text { Secondary outcomes } \\
\text { - Activities of daily living measured using the ADL Index } \\
\text { - Social function measured using the Social Functioning Examination }\end{array}$ \\
\hline Notes & $\begin{array}{l}\text { This trial had } 3 \text { arms (escitalopram, problem-solving therapy and placebo) but only the data from the } \\
\text { escitalopram group compared with placebo ( } n=117 \text { participants) are presented here }\end{array}$ \\
\hline
\end{tabular}

\section{Risk of bias}

\begin{tabular}{lll}
\hline Bias & Authors' judgement & Support for judgement \\
\hline $\begin{array}{l}\text { Random sequence genera- } \\
\text { tion (selection bias) }\end{array}$ & Low risk & $\begin{array}{l}\text { Quote: "... each block patients were randomly assigned 1 of the 3 treatments } \\
\text { using computer-generated random numbers of 1, 2, or 3 to escitalopram." pp. } \\
2393\end{array}$ \\
\hline $\begin{array}{l}\text { Allocation concealment } \\
\text { (selection bias) }\end{array}$ & Low risk & Quote: "all pills were identical ..." pp. 2393 \\
\hline $\begin{array}{l}\text { Blinding of participants } \\
\text { and personnel (perfor- } \\
\text { mance bias) } \\
\text { All outcomes }\end{array}$ & Low risk & Quote: "all pills were identical ..." pp. 2393 \\
\hline
\end{tabular}

$\begin{array}{ll}\begin{array}{l}\text { Blinding of outcome as- } \\ \text { sessment (detection bias) }\end{array} & \text { Low risk }\end{array} \quad \begin{aligned} & \text { Quote: "Patients were seen for evaluation by raters who were blinded to drug } \\ & \text { assignment ..." pp. } 2393\end{aligned}$

All outcomes

\begin{tabular}{|c|c|c|}
\hline $\begin{array}{l}\text { Incomplete outcome data } \\
\text { (attrition bias) }\end{array}$ & Low risk & $\begin{array}{l}\text { Comments: ITT analysis was reported. All participants were included in the } \\
\text { analysis despite dropouts }\end{array}$ \\
\hline
\end{tabular}
(attrition bias) 
Robinson 2008 (Continued)

Selective reporting (re- High risk Comments: did not report the results for anxiety porting bias)

Other bias

Low risk
Quote: "There were no significant differences between the groups in age, sex, years of education, marital status, or socioeconomic status."

"There were no significant inter-group differences in overall cumulative illness scores, coronary artery disease, low-density lipoprotein cholesterol, atrial fibrillation, chronic obstructive pulmonary disease, or systolic blood pressure. The patients randomized to escitalopram, however, had a significantly greater frequency of diabetes mellitus when compared with the placebo group" pp. 2395

\section{Study characteristics}

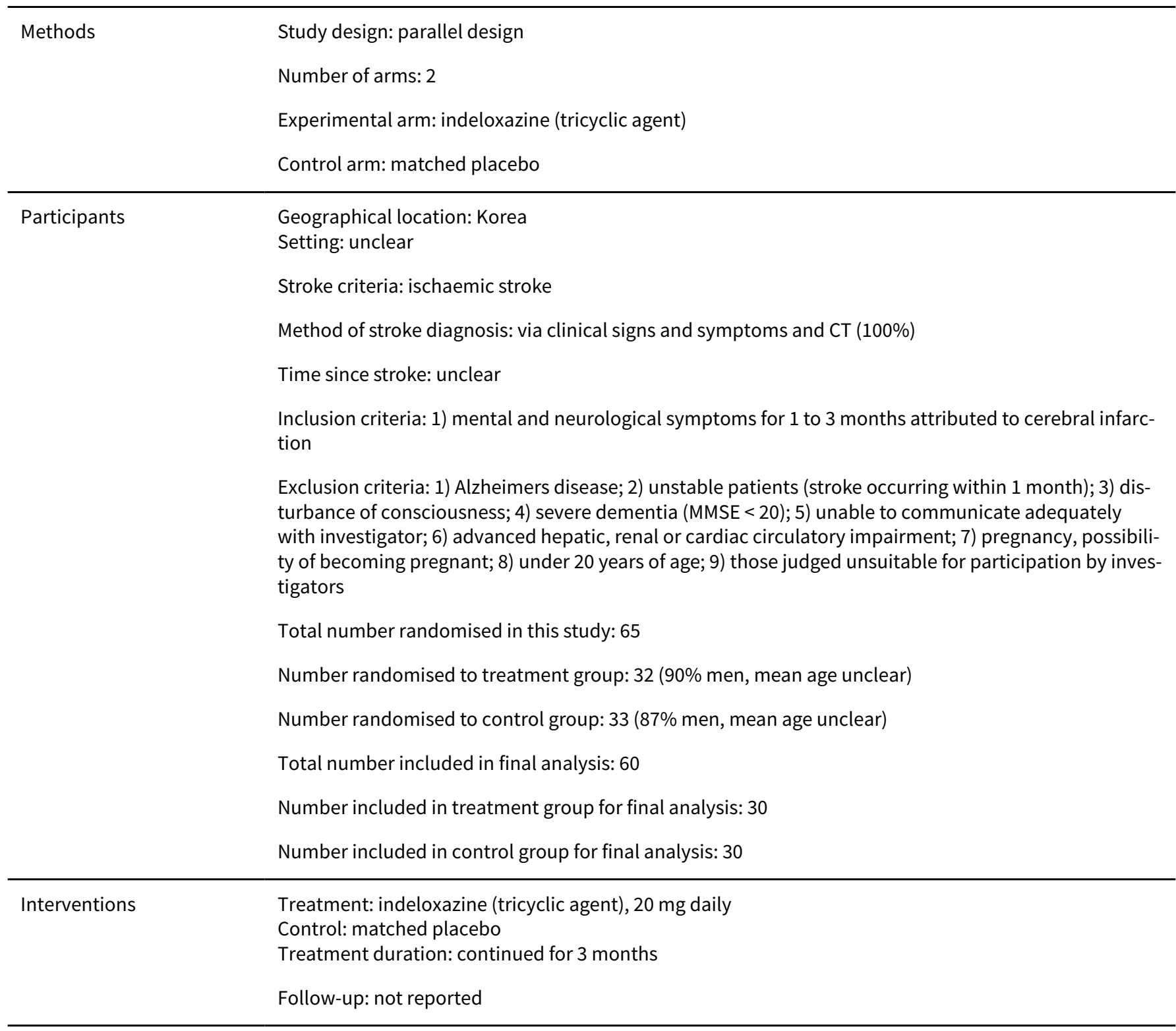


Roh 1996 (Continued)
Outcomes
Primary outcome
- Depression measured using the physician assessment of change in emotional disturbance from base- line to end of treatment

Notes

\section{Risk of bias}

\begin{tabular}{lll}
\hline Bias & Authors' judgement & Support for judgement \\
\hline $\begin{array}{ll}\text { Random sequence genera- } \\
\text { tion (selection bias) }\end{array}$ & Low risk & $\begin{array}{l}\text { Quote: "Patients were assigned by using a table of random numbers to receive } \\
\text { either } 20 \mathrm{mg} \text { of indeloxazine or placebo three times daily, after meals, for } 3 \\
\text { months." pp. } 633\end{array}$ \\
& &
\end{tabular}

\begin{tabular}{lll}
\hline $\begin{array}{l}\text { Allocation concealment } \\
\text { (selection bias) }\end{array}$ & Low risk & $\begin{array}{l}\text { Quote: "The placebo and test drug were given in identical tablet form to en- } \\
\text { sure double-masking." pp. 633-4 }\end{array}$ \\
\hline $\begin{array}{l}\text { Blinding of participants } \\
\begin{array}{l}\text { and personnel (perfor- } \\
\text { mance bias) }\end{array}\end{array}$ & Low risk & $\begin{array}{l}\text { Quote: "The placebo and test drug were given in identical tablet form to en- } \\
\text { sure double-masking." pp. 633-4 }\end{array}$ \\
\begin{tabular}{l} 
All outcomes \\
\hline
\end{tabular}
\end{tabular}

\begin{tabular}{lll}
\hline $\begin{array}{l}\text { Blinding of outcome as- } \\
\text { sessment (detection bias) } \\
\text { All outcomes }\end{array}$ & Low risk & $\begin{array}{l}\text { Quote: "The placebo and test drug were given in identical tablet form to en- } \\
\text { sure double-masking." pp. 633-4 }\end{array}$ \\
\hline $\begin{array}{l}\text { Incomplete outcome data } \\
\begin{array}{l}\text { (attrition bias) } \\
\text { All outcomes }\end{array}\end{array}$ & High risk & $\begin{array}{l}\text { Quote: "Of the } 33 \text { patients in the placebo group, } 2 \text { patients complained of } \\
\text { headache and } 1 \text { showed a deterioration in eyesight: these patients withdrew } \\
\text { from the study and were not included in the analysis." pp. } 636-7\end{array}$ \\
\hline $\begin{array}{l}\text { Selective reporting (re- } \\
\text { porting bias) }\end{array}$ & Unclear risk & Comments: no trial protocol available to compare with the publication \\
\hline $\begin{array}{l}\text { Other bias } \\
\text { Low risk }\end{array}$ & Lomments: there was no statistically significant difference between the treat- \\
\hline
\end{tabular}

Tsai 2011

\section{Study characteristics}

\begin{tabular}{ll}
\hline Methods & Study design: parallel design \\
& Number of arms: 2 \\
& Experimental arm: milnacipran (SNRI) \\
Control arm: matching placebo & \\
\hline Participants & Geographical location: Taiwan \\
& Setting: inpatient \\
& Stroke criteria: first ischaemic or recurrent stroke \\
& Method of stroke diagnosis: confirmed by imaging \\
& Time since stroke: 4 weeks before admission \\
Inclusion criteria: 1 ) first or recurrent ischaemic stroke & Exclusion criteria: 1 ) with past history of depression, substance abuse or psychosis; 2) taking antide- \\
pressants at least two weeks before stroke; 3) MMSE < 15; 4) impairment of communication; 5) TIA; 6) \\
had possible concurrent depression (HDRS > 10).
\end{tabular}


Tsai 2011 (Continued)

Depression criteria: HDRS $>10$

Total number randomised in this trial: 92

Number randomised to treatment group: 46 (65.2\% men, mean age 61.0, SD 10.8)

Number randomised to control group: 46 (63\% men, mean age 64.9, SD 10.5)

Total number included in final analysis: 56

Number included in treatment group for final analysis: 25

Number included in control group for final analysis: 31

\begin{tabular}{ll}
\hline Interventions & $\begin{array}{l}\text { Treatment: milnacipran (SNRI), } 50 \mathrm{mg} / \text { day titrated to } 100 \mathrm{mg} / \text { day } \\
\text { Control: matching placebo } \\
\text { Treatment duration: } 12 \text { months } \\
\text { Follow-up: not reported }\end{array}$ \\
\hline Outcomes & Primary outcomes \\
- Depression measured using the HDRS \\
Secondary outcomes \\
- Quality of life measured using the QoL Index \\
- Disability measured using the London Handicap Scale
\end{tabular}

Notes

\section{Risk of bias}

Bias Authors' judgement Support for judgement

Random sequence genera- Unclear risk tion (selection bias)

Quote: "The randomization was carried out by our pharmacy department, which has a professional team in charge of the clinical drug trials in our hospital." pp. 264

Comments: method of sequence generation not reported

\begin{tabular}{ll}
\hline $\begin{array}{l}\text { Allocation concealment } \\
\text { (selection bias) }\end{array}$ & Qow risk \\
pant based on their random assignment." pp. 264
\end{tabular}

\begin{tabular}{|c|c|c|}
\hline $\begin{array}{l}\text { Blinding of participants } \\
\text { and personnel (perfor- }\end{array}$ & Low risk & $\begin{array}{l}\text { Quote: "The placebo was tailor-made, and the appearance and weight (starch } \\
\text { inside) were the same as that of the active drug, milnacipran." pp. } 265\end{array}$ \\
\hline
\end{tabular}
and personnel (perforinside) were the same as that of the active drug, milnacipran." pp. 265

mance bias)

All outcomes

\begin{tabular}{|c|c|c|}
\hline $\begin{array}{l}\text { Blinding of outcome as- } \\
\text { sessment (detection bias) } \\
\text { All outcomes }\end{array}$ & Low risk & Quote: "All the interviewers were blinded to the patient's medication." pp. 26 \\
\hline $\begin{array}{l}\text { Incomplete outcome data } \\
\text { (attrition bias) } \\
\text { All outcomes }\end{array}$ & Low risk & $\begin{array}{l}\text { Quote: "Fifty-six (60.9\%) of } 92 \text { patients completed } 12 \text { months of study treat- } \\
\text { ment: } 25(54.3 \%) \text { of } 46 \text { in the treatment group and } 31(67.4 \%) \text { of } 46 \text { in the } \\
\text { placebo group, respectively." pp. } 265\end{array}$ \\
\hline
\end{tabular}

Comments: Figure 1 and Table 1 presents ITT analysis. All 92 participants were included in the analysis

$\begin{array}{ll}\begin{array}{l}\text { Selective reporting (re- } \\ \text { porting bias) }\end{array} & \text { Quote: "The modified version of the 17-item HDRS without item } 14 \text { (sexual be- } \\ & \begin{array}{l}\text { havior), NIHSS, and BI, were assessed in each of the follow-up visits. The defin- } \\ \text { ition of PSD in the study was based on the diagnostic criteria of major depres- } \\ \text { sive episode in DSM-IV by professional psychiatrists." }\end{array}\end{array}$


Tsai 2011 (Continued)

Other bias

Unclear risk
Comments: treatment group appears to contain higher proportion of participants who have higher than secondary school education $34.8 \%$ compared to $20.0 \%$

Watkins 2007

\section{Study characteristics}

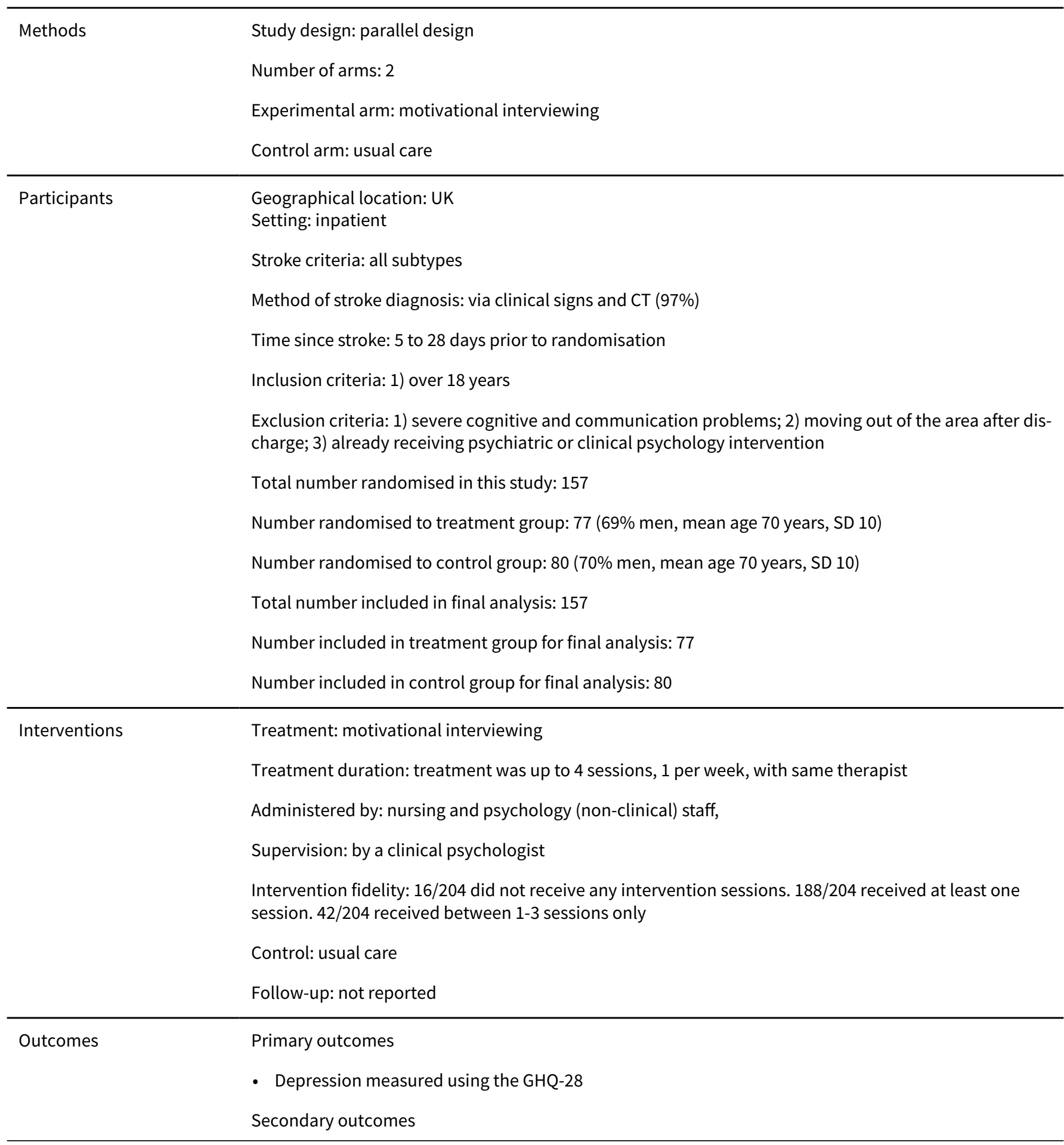

Pharmacological, psychological and non-invasive brain stimulation interventions for preventing depression after stroke (Review) 
Watkins 2007 (Continued)

- Depression measured using the Yale Depression Screen

- Activities of daily living measured using the BI

Notes Additional unpublished data provided by authors

\section{Risk of bias}

\begin{tabular}{lll}
\hline Bias & Authors' judgement & Support for judgement \\
\hline $\begin{array}{ll}\text { Random sequence genera- } \\
\text { tion (selection bias) }\end{array}$ & Unclear risk & $\begin{array}{l}\text { Quote: "A research nurse randomized patients (1:1 ratio) to either usual care } \\
\text { (control) or MI (intervention) using minimization over sex, age (65 and 65 }\end{array}$ \\
& & years), baseline function in activities of daily living (ADL; Barthel: 18 to 20; 11 to \\
& & $17 ; 0$ to 10), 24 and location (acute stroke unit or not)." pp. 1957
\end{tabular}

17; 0 to 10),24 and location (acute stroke unit or not)." pp. 1957

Comments: method of sequence generation not reported.

\begin{tabular}{|c|c|c|}
\hline $\begin{array}{l}\text { Allocation concealment } \\
\text { (selection bias) }\end{array}$ & High risk & $\begin{array}{l}\text { Quote: "The same nurse then assigned intervention group patients to } 1 \text { of } 4 \\
\text { therapists using an opaque sealed envelope in a pseudo-randomized blocked } \\
\text { design." pp. } 1957\end{array}$ \\
\hline
\end{tabular}
design." pp. 1957

Blinding of participants High risk and personnel (performance bias)

All outcomes

\section{Blinding of outcome as- High risk} sessment (detection bias) All outcomes
Quote: "The therapists were not involved in the initial assessment of patients, randomization, or assignment of patients to therapists." pp. 1957

Comments: the same research nurse randomised and assigned patients to therapists

Quote: "Surviving patients were sent a questionnaire. Patients not returning questionnaires within 2 weeks were telephoned by a second research nurse, blind to group allocation, and given the option of declining, having a further questionnaire posted, completing the questionnaire over the telephone, or receiving a home visit to assist." pp. 1958

Comments: Figure 1 shows the trial profile. Under analysis, it states that all participants were included in the analysis. Missing data were imputed

Incomplete outcome data Low risk

(attrition bias)

Low risk

All outcomes

\begin{tabular}{lll}
$\begin{array}{l}\text { Selective reporting (re- } \\
\text { porting bias) }\end{array}$ & Unclear risk & Comments: no trial protocol available to compare with the publication \\
\hline Other bias & Low risk & $\begin{array}{l}\text { Comments: there was no statistically significant difference between the treat- } \\
\text { ment and control group in baseline demographic characteristics }\end{array}$ \\
\hline
\end{tabular}

Wichowicz 2017

\section{Study characteristics}

$\begin{array}{ll}\text { Methods } & \text { Study design: parallel design } \\ & \text { Number of arms: } 2 \\ & \text { Experimental arm: solution focused brief therapy } \\ \text { Control arm: no psychotherapeutic interventions }\end{array}$

Geographical location: Poland
Setting: inpatient
Stroke criteria: first ischaemic stroke
Method of stroke diagnosis: confirmed by imaging


Wichowicz 2017 (Continued)

Time since stroke: 30 days

Inclusion criteria: 1) first ischaemic stroke

Exclusion criteria: 1) extensive intellectual defects precluding verbal contact, dementia (MMSE $<23$

points); 2) aphasia; 3 ) severe dysarthria; 4) severe paresis; 5) NIHSS scale $>21$; (6) aged $>65$ years

Depression criteria: depression score not an entry criteria

Total number randomised in this trial: 100

Number randomised to treatment group: 51 (\% men not reported, mean age 53.3, SD 9.9)

Number randomised to control group: 49 (\% men not reported, mean age 54.2, SD 9.4)

Total number included in final analysis: 62

Number included in treatment group for final analysis: 30

Number included in control group for final analysis: 32

Interventions
Treatment: solution focused brief therapy
Administered by: a therapist
Supervision: not reported
Intervention fidelity: $30 / 51$ received allocated inte
tion. $32 / 49$ received control intervention, $17 / 49$ did
Control: no therapeutic interventions
Follow-up: not reported
Primary outcomes
- Depression measured using the HADS
- Anxiety measured using the HADS
Secondary outcomes
- Disability measured using the RS
- Activities of daily living measured using the BI
- Impairment measured using the NIHSS

Notes

\section{Risk of bias}

Bias Authors' judgement Support for judgement

Random sequence genera- Low risk Quote: "They were assigned by simple random allocation to one of two tion (selection bias) groups..." pp. 1508

Comments: sequence generation was done through random number tables (according to the study author)

\begin{tabular}{|c|c|c|}
\hline $\begin{array}{l}\text { Allocation concealment } \\
\text { (selection bias) }\end{array}$ & Unclear risk & $\begin{array}{l}\text { Quote: "Allocation sequence was concealed until participation was pro- } \\
\text { posed." pp. } 1508\end{array}$ \\
\hline
\end{tabular}

Comments: method of allocation concealment not reported

\begin{tabular}{|c|c|c|}
\hline $\begin{array}{l}\text { Blinding of participants } \\
\text { and personnel (perfor- }\end{array}$ & High risk & $\begin{array}{l}\text { Comments: participants were not blinded according to the author. Personnel } \\
\text { were not blinded according to the author }\end{array}$ \\
\hline
\end{tabular}

$$
\text { mance bias) }
$$

All outcomes 
Wichowicz 2017 (Continued)

Blinding of outcome as- Low risk Quote: "All assessments were conducted and interpreted by one researcher sessment (detection bias) who was blinded to the group and identity of subjects." pp. 1509

All outcomes

$\begin{aligned} & \text { Incomplete outcome data } \\ & \text { (attrition bias) }\end{aligned}$
$\begin{aligned} & \text { Cow risk } \\ & \text { tion were included in the analysis }\end{aligned}$

All outcomes

tion were included in the analysis

Selective reporting (re- Unclear risk Comments: no trial protocol available to compare with the publication
porting bias)

porting bias)

Other bias Low risk

Quote: "There were no significant differences in the age, education and the physical function between the groups as shown in Table 1." pp. 1509

Comments: no other bias detected

Xu 2006

\section{Study characteristics}

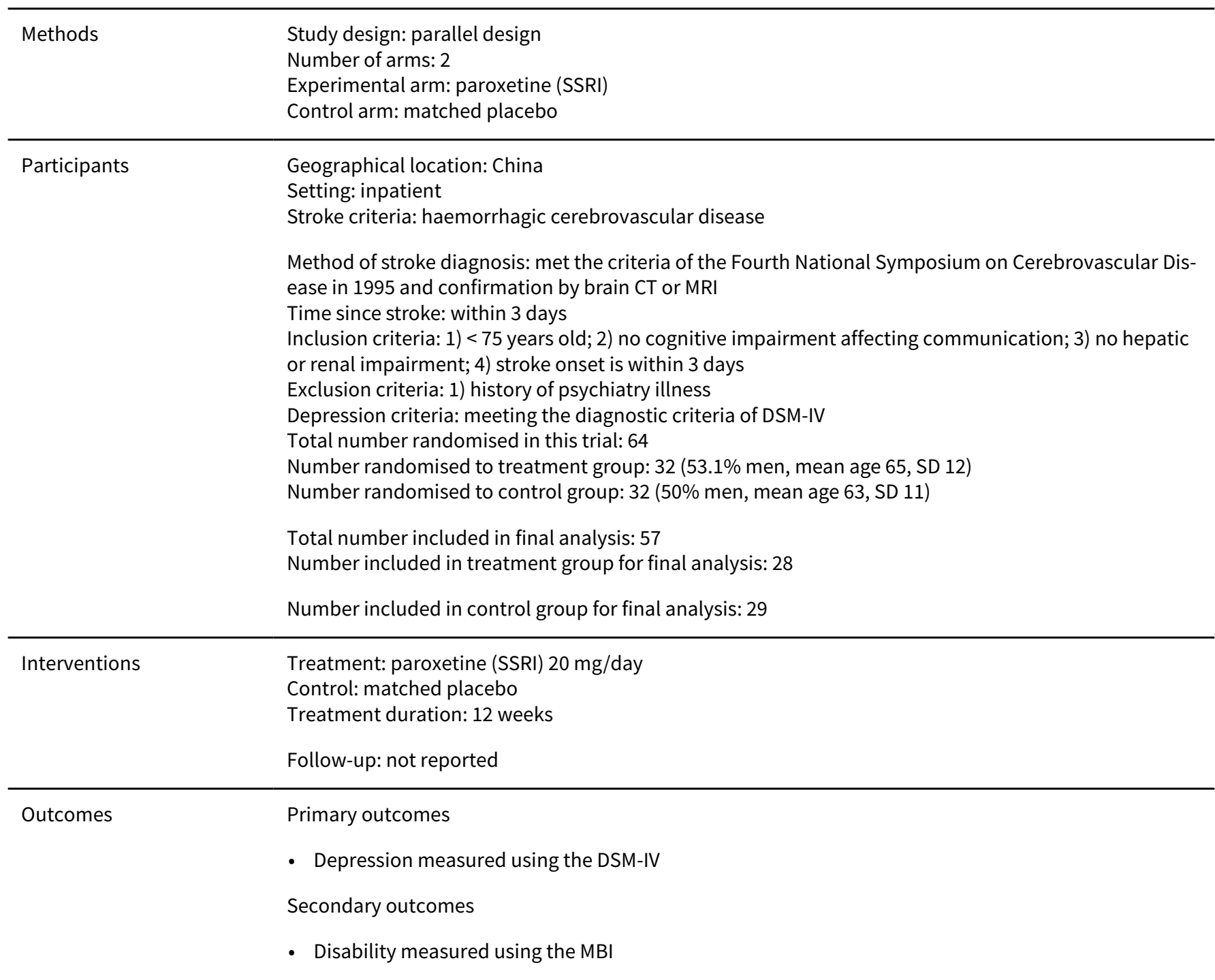


Notes

\section{Risk of bias}

\begin{tabular}{|c|c|c|}
\hline Bias & Authors' judgement & Support for judgement \\
\hline $\begin{array}{l}\text { Random sequence genera- } \\
\text { tion (selection bias) }\end{array}$ & Unclear risk & Comments: method of sequence generation not reported \\
\hline $\begin{array}{l}\text { Allocation concealment } \\
\text { (selection bias) }\end{array}$ & Unclear risk & Comments: method of allocation concealment not reported \\
\hline $\begin{array}{l}\text { Blinding of participants } \\
\text { and personnel (perfor- } \\
\text { mance bias) } \\
\text { All outcomes }\end{array}$ & Unclear risk & Comments: blinding of participants and personnel not reported \\
\hline $\begin{array}{l}\text { Blinding of outcome as- } \\
\text { sessment (detection bias) } \\
\text { All outcomes }\end{array}$ & Unclear risk & Comments: blinding of outcome assessors not reported \\
\hline $\begin{array}{l}\text { Incomplete outcome data } \\
\text { (attrition bias) } \\
\text { All outcomes }\end{array}$ & Unclear risk & $\begin{array}{l}\text { Comments: ITT analysis reported but } 7 / 64 \text { dropped out, how missing data } \\
\text { were handled was not reported }\end{array}$ \\
\hline $\begin{array}{l}\text { Selective reporting (re- } \\
\text { porting bias) }\end{array}$ & Unclear risk & Comments: no trial protocol available to compare with the publication \\
\hline Other bias & Low risk & $\begin{array}{l}\text { Comments: no differences in age, gender, Modified Edinburgh Stroke Scale or } \\
\text { ADL scales }\end{array}$ \\
\hline
\end{tabular}

*: control group numbers and values halved to enable comparison with each active intervention

$\wedge$ : results for attention control and control group pooled

ADL: activities of daily living

BDI: Beck Depression Inventory

BI: Barthel Index

BOP: Beoordelingsschaal voor Oudere Patiënten (also known as Behavioural Rating Scale)

CES-D: Center for Epidemiologic Studies-Depression scale

CGI: Clinical Global Impression of Depression

CT: computed tomography

CVA: cerebrovascular accident

DSM-IV: Diagnostic and Statistical Manual of Mental Disorders- Fourth Edition

FIM: Functional Independence Measure

GDS: Geriatric Depression Scale

GHQ-28: 28-item General Health Questionnaire

HADS: Hospital Anxiety and Depression Scale

HDRS: Hamilton Depression Rating Scale

HSS: Hemispheric Stroke Scale

ICD-10: International Classification of Diseases, Tenth Revision

IM: intramuscular

ITT: intention-to-treat

IQR: interquartile range

MADRS: Montgomery-Åsberg Depression Rating Scale

MBI: Modified Barthel Index

MCA: middle cerebral artery

mRS: modified Rankin Scale

MMSE: Mini Mental State Examination

Pharmacological, psychological and non-invasive brain stimulation interventions for preventing depression after stroke (Review) 
MRI: magnetic resonance imaging

PHQ-9: 9-item Patient Health Questionaire

QoL: quality of life

RS: Rankin Scale

SD: standard deviation

SE: standard error

SNRI: serotonin and norepinephrine reuptake inhibitors

SSRI: selective serotonin reuptake inhibitors

TIA: transient ischaemic attack

ZDS: Zung Depression Scale

Characteristics of excluded studies [ordered by study ID]

\begin{tabular}{|c|c|}
\hline Study & Reason for exclusion \\
\hline Aben 2014 & Depression is not the primary outcome of the study \\
\hline Agnoli 1985 & Unable to isolate data for participants with stroke only \\
\hline Bramanti 1989 & Results not available in suitable format for the review \\
\hline Casella 1960 & Depression is not the primary outcome of the study \\
\hline Choi-Kwon 2006 & Unable to isolate outcome data for non- depressed participants at randomisation \\
\hline Downes 1995 & Unable to isolate outcome data for non- depressed participants at randomisation \\
\hline F.X. 1976 & Unable to isolate participants with stroke only \\
\hline Friedland 1992 & Results not available in suitable format for the review \\
\hline Graffingo 2003 & Results not available \\
\hline Hadidi 2014 & Unable to isolate outcome data for non- depressed participants at randomisation \\
\hline ljzerman 2005 & Depression is not the primary outcome of this study \\
\hline Isenberg 2000 & Results not available \\
\hline Kim 2017 & Unable to isolate outcome data for non-depressed participants at randomisation \\
\hline Kim 2017a & Participants were depressed at baseline \\
\hline Laska 2005 & Depression is not the primary outcome of this study \\
\hline Latow 1983 & Results not available \\
\hline Leathley 2003 & Results not available in format suitable for this review \\
\hline Leijon 1989 & Depression is not the primary outcome of this study \\
\hline Lobjanidze 2010 & Depression is not the primary outcome of this study \\
\hline Martucci 1986 & Unable to isolate data for participants with stroke only \\
\hline McCafferty 2000 & Results not available \\
\hline
\end{tabular}




\begin{tabular}{ll}
\hline Study & Reason for exclusion \\
\hline Ohtomo 1985 & Results not available \\
\hline Ostwald 2006 & No usual care comparison \\
\hline Ostwald 2014 & Unable to isolate outcome data for non- depressed participants at randomisation \\
\hline Otomo 1986 & Unclear if depression is the primary outcome of the study \\
\hline Poppitt 2009 & Intervention did not meet review criteria \\
\hline Raffaele 1996 & Unable to isolate outcome data for non- depressed participants at randomisation \\
\hline Sasaki 2017 & Depression is not the primary outcome of this study \\
\hline Sivenius 2001 & Depression is not the primary outcome of this study \\
\hline Walker-Batson 1995 & Depression is not the primary outcome of this study \\
\hline Yamamoto 1999 & Unclear if depression is the primary outcome of the study \\
\hline
\end{tabular}

Characteristics of studies awaiting classification [ordered by study ID]

\section{Chang 2011}

Study design: parallel design
Number of arms: 2
Treatment arm: rational emotive behaviour therapy (REBT) + regular care
Control arm: prescribed medications (i.e. antithrombotic, vasodilators, neurotrophic drugs) and re-
habilitation training programs for physical functioning

\section{Participants}

Geographical location: China

Setting: inpatient

Stroke criteria: patients with hemiplegic stroke

Method of stroke diagnosis: based on the diagnostic criteria set at the National Fourth Cerebrovascular Disease Conference. Each patient's condition was confirmed with a CT or MRI scan

Time since stroke: less than 2 weeks

Inclusion criteria: 1) patients with hemiplegic stroke, 2) patients who were less than 2 weeks poststroke

Exclusion criteria: 1) MMSE score of < 24, 2) severe aphasia, 3) those who failed to complete the treatment, including those who died or stopped the treatment by choice, 4) history of psychiatric illness

Depression criteria: Chinese version of the HDRS. Mean depression score used rather than depression cut off

Total number randomised in this trial: 77

Number randomised in treatment group: 39 
Number randomised to control group: 38

Total number included in final analysis: 66

Number included in treatment group for final analysis: 34

Number included in control group for final analysis: 32

Interventions

Treatment: REBT + regular care. Counselling, which consisted of a knowledge component: education about health psychology and recovery from hemiplegic stroke; and a behavioural training component including belief changes forgiveness training and anger management

Treatment duration: 1-2 hours weekly for 1 month

Administerd by: treatment group: by trained psychology graduate; regular care: administered by the hospital nurses

Supervision: not reported

Intervention fidelity: those who failed to complete the intervention were excluded from the study. $34 / 39$ completed in the treatment arm. 32/38 completed in the control arm

Control: prescribed medications (i.e. antithrombotic, vasodilators, neurotrophic drugs) and rehabilitation training programs for physical functioning

Follow-up: not reported

Outcomes

Primary outcomes

- Anger management measured using the State Trait Anger Expression Inventory (STAXI)

- Anxiety measured using the Chinese version of the HDRS

- Depression measured using the Chinese version of the HDRS

Secondary outcomes

- Activities of daily living measured using the Chinese version of the BI

- Quality of life measured using the Chinese version of the Stroke Specific Quality of Life scale

Notes

Evans 1985

Methods Study design: parallel design

Number of arms: 2

Treatment arm: telephone counselling

Control arm: usual care

Participants

Geographical location: USA

Setting: outpatient

Stroke criteria: unclear (also includes people with spinal cord injury, CNS disease and 'other')

Method of stroke diagnosis: not reported

Time since stroke: not reported 
Inclusion criteria: 1) clients discharged from rehabilitation centre; 2) housebound; 3) able to hear; 4) ordinary speech; and 5) sufficient cognitive ability to engage in meaningful conversation

Exclusion criteria: not reported

Depression criteria: score taken from the Life satisfaction Index (LSI), unclear how scored

Total number randomised in this trial: 38

Number randomised in treatment group: 19 (95\% men, mean age 54.8 years, SD 11.9 years) - 4 stroke participants

Number randomised to control group: 19 ( $95 \%$ men, mean age 54.8 years, SD 10.2 years) - 5 stroke participants

Total number included in final analysis: unclear

Number included in treatment group for final analysis: unclear

Number included in control group for final analysis: unclear

Interventions

Treatment: 8 weekly hour-long counselling sessions by phone with groups of 4 clients. Formulation of behaviourally-specific goals was encouraged and developed with each client and discussion was directed at finding ways to meet those goals

Administered by: an experienced counsellor

Supervision: not reported

Intervention fidelity: not reported

Control: usual care (no contact)

Treatment duration: not reported

Follow-up: not reported

Outcomes

Primary outcome

- Depression using Life satisfaction Index (LSI)

Notes

Unable to obtain any more information on this trial or series of trials despite multiple attempts since 2003

\section{IRCT201112228490N1}

\section{Methods}

Study design: parallel design

Number of arms: 2

Treatment arm: fluoxetine (SSRI)

Control arm: matched placebo

\section{Participants}

Setting: unclear

Stroke criteria: cerebral ischaemic stroke

Method of stroke diagnosis: unclear 
Inclusion criteria: 1) informed consent; 2) unilateral occlusion of MCA trunk; 3) resident in Rasht; 4) admission NIHSS < 20; 5) no history of alcohol abuse; 6) no history of insomnia, epilepsy; 7) no previous history of cerebral haemorrhage and heart of cerebral stroke; 8) no previous history of systemic diseases of other organs, including liver failure and kidney; 9) absence of cardiac pace maker, severe neuropathy, systemic vascular disease or major affective disorders; 10) absence of concomitant stroke in an area other than the stroke of the MCA

Exclusion criteria: 1) dissatisfaction of patient during the study; 2) occurrence of serious adverse drug affects at any time during drug administration; 3 ) alcohol abuse during the study period; 4) occurrence of post stroke depression concomitant use of the mono amine oxidase inhibitors or serotonergic drugs such as tricyclic antidepressants and SSRI

Depression criteria: none

Total number randomised in this trial: unclear

Number randomised in treatment group: unclear

Number randomised to control group: unclear

Total number included in final analysis: unclear

Number included in treatment group for final analysis: unclear

Number included in control group for final analysis: unclear

Control: matched placebo $15 \mathrm{mg}$ for the first month and $20 \mathrm{mg}$ for the next 2 months

Treatment duration: 3 months

Follow-up: unclear

Outcomes

Primary outcomes

- Disability measured using the mRS

- Activities of daily living measured using the BI

- Functional recovery measured using the NIHSS

- Depression measured using the BDI

Secondary outcomes

- Cerebral blood flow changes of MCA measured by the Transcranial Doppler

Notes

\section{Katz 1998}

\section{Methods}

Study design: unclear

Number of arms: unclear

Treatment arm: group psychotherapy/ behavioural therapy/ antidepressants - combination unclear

Control arm: unclear 
Katz 1998 (Continued)

\author{
Setting: unclear \\ Stroke criteria: unclear \\ Method of stroke diagnosis: unclear \\ Time since stroke: unclear \\ Inclusion criteria: unclear \\ Exclusion criteria: unclear \\ Depression criteria: unclear
}

Total number randomised in this trial: unclear

Number randomised in treatment group: unclear

Number randomised to control group: unclear

Total number included in final analysis: unclear

Number included in treatment group for final analysis: unclear

Number included in control group for final analysis: unclear

Treatment: group psychotherap
Administered by: unclear
Supervision: unclear
Intervention fidelity: unclear
Control: unclear
Treatment duration: unclear
Follow-up: unclear

Outcomes Primary outcomes

- Depression (unclear what measure was used)

Secondary outcomes

- Cognitive impairment (unclear what measure was used)

- Functional recovery (unclear what measure was used)

\title{
Razazian 2016
}

\begin{tabular}{ll}
\hline Methods & Study design: parallel design \\
Number of arms: 2 \\
Treatment arm: fluoxetine (SSRI) \\
Control arm: matched placebo
\end{tabular}

\section{Participants}

Geographical location: Iran 
Razazian 2016 (Continued)

Setting: inpatient

Stroke criteria: acute Ischemic stroke, in MCA region

Method of stroke diagnosis: confirmed by radiological imaging (CT)

Time since stroke: not reported

Inclusion criteria: 1) stroke in MCA region that leads to monoparesis, hemiparesis or hemiplegia, 2) stable condition

Exclusion criteria: 1) death, 2) pregnancy, 3) poor compliance with drugs and physiotherapy, 4) potential drug complications, 5) metabolic disease, 6) stroke in anterior cerebral artery or posterior cerebral artery

Depression criteria: not reported.

Total number randomised in this trial: 172

Number randomised in treatment group: 86

Number randomised to control group: 86

Total number included in final analysis: 150

Number included in treatment group for final analysis: 75

Number included in control group for final analysis: 75

Treatment: fluoxetine (SSRI) 20 mg once per day orally
Control: matched placebo
Treatment duration: 90 days
Follow-up: not reported

Outcomes

Primary outcome

- Depression measured using the HDRS

Secondary outcomes

- Activities of daily living measured using the $\mathrm{BI}$

- Neurological examinations

\section{Notes}

BDI: Beck Depression Inventory

BI: Barthel Index

CNS: central nervous system

$\mathrm{CT}$ : computed tomography

HDRS: Hamilton Depression Rating Scale

MCA: middle cerebral artery

MMSE: Mini Mental State Examination

MRI: magnetic resonance imaging

mRS: Modified Rankin Scale

NIHSS: National Institute of Health Stroke Scale

SD: standard deviation

SSRI: Selective Serotonin Reuptake Inhibitors

USA: United States of America 
Characteristics of ongoing studies [ordered by study ID]

Kirkevold 2018

Study name

Promoting psychosocial well-being following stroke: study protocol for a randomized, controlled trial

Methods

Study design: parallel design

Number of arms: 2

Treatment arm: dialogue-based intervention to promote psychosocial well-being

Control arm: usual care

Participants

Geographical location: Norway

Setting: outpatient

Stroke criteria: unclear

Method of stroke diagnosis: unclear

Time since stroke: unclear

Inclusion criteria: unclear

Exclusion criteria: unclear

Depression criteria: unclear

Total number randomised in this trial: unclear

Number randomised in treatment group: unclear

Number randomised to control group: unclear

Total number included in final analysis: unclear

Number included in treatment group for final analysis: unclear

Number included in control group for final analysis: unclear

Treatment: the intervention consists of 8 one-to-one and a half hour dialogue-based sessions between the stroke survivor and a specially trained health professional (RN or OT). Each meeting has a guiding topical outline, which addresses significant issues described in the research literature (e.g. bodily changes, emotional challenges, personal relations, daily life issues, meaningful activities, existential issues, important values, etc)

Administered by: a specially trained health professional (RN or OT)

Supervision: not reported

Intervention fidelity: not reported

Control: the control group receives treatment as usual and no intervention beyond participation in the assessment interviews at 1, 6, and 12 months. As participants are recruited from a variety of settings (acute stroke care and rehabilitation units), we anticipate some variation in the usual care provided

Treatment duration: 8 sessions

Follow-up: 6- and 12-month assessments 
Kirkevold 2018 (Continued)

- Depressive symptoms and anxiety (measured by GHQ-28)

Secondary outcomes

- Sense of coherence measured using the SOC-13

- Health-related quality of life measured using the SAQOL-39

\begin{tabular}{ll}
\hline Starting date & December 2014 \\
\hline Contact information & E: marit.kirkevold@medisin.uio.no; \\
& $\begin{array}{l}\text { Institute of Health and Society and Research Center for Rehabilitation and Rehabilitation services } \\
\text { and models (CHARM), University of Oslo, P.O.Box 1130, Blindern, 0318 Oslo, Norway }\end{array}$ \\
\hline
\end{tabular}

Notes

Sitzer 2002

Study name Prevention of poststroke depression after acute ischemic stroke using the selective serotonine reuptake inhibitor sertraline: "PreDIS-Study"

\begin{tabular}{ll}
\hline Methods & Study design: parallel design \\
& Number of arms: 2 \\
Treatment arm: sertraline (SSRI) \\
Control arm: matched placebo
\end{tabular}

\section{Participants}

Geographical location: Germany

Setting: mixed

Stroke criteria: unilateral ischaemic stroke

Method of stroke diagnosis: unclear

Time since stroke: 3 to 6 days prior to randomisation

Inclusion criteria: unclear

Exclusion criteria: 1) early and complete recovery from neurological symptoms; 2) mechanically ventilated for more than 2 days; 3 ) severe aphasia or dementia; 4) already on antidepressant

Depression criteria: none

Total number randomised in this trial: unclear

Number randomised in treatment group: unclear

Number randomised to control group: unclear

Total number included in final analysis: unclear

Number included in treatment group for final analysis: unclear

Number included in control group for final analysis: unclear 
Sitzer 2002 (Continued)

Treatment duration: treatment to continue for 6 months

Follow-up: unclear

Primary outcomes
- Depression measured using the HADS, MADRS and ICD-10 diagnostic criteria
Secondary outcomes
- Activities of daily living measured using the BI
- Functional capacity measured using the mRS
- Cognitive function measured using the MMSE
- Impairment measured using the European Stroke Scale
- Quality of life measured using the SF-36

\begin{tabular}{ll}
\hline Starting date & August 2001 \\
\hline Contact information & $\begin{array}{l}\text { Dr. Matthias Sitzer, Zentrum der Neurologie und Neurochirurgie JW Goethe-Universitat Frankfurt } \\
\text { am Main Schleusenweg 2-16 D-60528 Frankfurt am Main Germany, E: sitzer@em.uni-frankfurt.de }\end{array}$
\end{tabular}

Notes

BI: Barthel Index

GHQ-28: 28-item General Health Questionnaire

HADS: Hospital Anxiety and Depression Scale

ICD-10: International Classification of Disease- 10

MADRS: Montgomery Asberg Depression Rating Scale

MMSE: Mini Mental State Examination

mRS: modified Rankin Scale

OT: occupational therapist

$\mathrm{RN}$ : registered nurse

SAQOL-39: 39-item Stroke Aphasia Quality of Life Scale

SF-36: 36-item Short Form Questionnaire

SOC-13: 13-item Sense of Coherence Scale

SSRI: selective serotonin reuptake inhibitors

DATA AND ANALYSES

Comparison 1. Pharmacological interventions (antidepressants) versus placebo

\begin{tabular}{|c|c|c|c|c|}
\hline Outcome or subgroup title & $\begin{array}{l}\text { No. of } \\
\text { studies }\end{array}$ & $\begin{array}{l}\text { No. of } \\
\text { partici- } \\
\text { pants }\end{array}$ & Statistical method & Effect size \\
\hline $\begin{array}{l}\text { 1.1 Depression: meeting study criteria for de- } \\
\text { pression at end treatment }\end{array}$ & 9 & 734 & Risk Ratio (M-H, Random, 95\% Cl) & $0.50[0.37,0.68]$ \\
\hline 1.1.1 CGI (high score = more severe) & 1 & 137 & Risk Ratio (M-H, Random, 95\% Cl) & $0.62[0.34,1.15]$ \\
\hline $\begin{array}{l}\text { 1.1.2 Clinician interview/impression (no im- } \\
\text { provement) }\end{array}$ & 1 & 65 & Risk Ratio (M-H, Random, 95\% Cl) & $0.45[0.26,0.79]$ \\
\hline 1.1.3 Meets DSM-III-R criteria & 3 & 148 & Risk Ratio (M-H, Random, 95\% Cl) & $0.62[0.27,1.42]$ \\
\hline
\end{tabular}




\begin{tabular}{|c|c|c|c|c|}
\hline Outcome or subgroup title & $\begin{array}{l}\text { No. of } \\
\text { studies }\end{array}$ & $\begin{array}{l}\text { No. of } \\
\text { partici- } \\
\text { pants }\end{array}$ & Statistical method & Effect size \\
\hline 1.1.4 Meets DSM-IV criteria & 2 & 181 & Risk Ratio (M-H, Random, 95\% Cl) & $0.33[0.15,0.69]$ \\
\hline 1.1.5 HADS (high score = more severe) & 1 & 111 & Risk Ratio (M-H, Random, 95\% Cl) & $0.74[0.32,1.70]$ \\
\hline 1.1.6 HDRS (high score = more depressed) & 1 & 92 & Risk Ratio (M-H, Random, 95\% Cl) & $0.14[0.02,1.12]$ \\
\hline $\begin{array}{l}\text { 1.2 Scoring above cut-off points for a depres- } \\
\text { sive disorder at end of treatment }\end{array}$ & 0 & & Risk Ratio (M-H, Random, 95\% Cl) & $\begin{array}{l}\text { Totals not select- } \\
\text { ed }\end{array}$ \\
\hline $\begin{array}{l}\text { 1.3 Depression: average change in scores be- } \\
\text { tween baseline and end of treatment }\end{array}$ & 5 & 211 & $\begin{array}{l}\text { Std. Mean Difference (IV, Random, } \\
95 \% \mathrm{CI})\end{array}$ & $-0.05[-0.33,0.23]$ \\
\hline 1.3.1 HDRS (high score = more depressed) & 4 & 100 & $\begin{array}{l}\text { Std. Mean Difference (IV, Random, } \\
95 \% \mathrm{CI})\end{array}$ & $-0.16[-0.58,0.26]$ \\
\hline 1.3.2 HADS (high score = more depressed) & 1 & 111 & $\begin{array}{l}\text { Std. Mean Difference (IV, Random, } \\
95 \% \mathrm{CI})\end{array}$ & $0.04[-0.34,0.41]$ \\
\hline $\begin{array}{l}\text { 1.4 Depression: mean scores at end of treat- } \\
\text { ment }\end{array}$ & 4 & 100 & $\begin{array}{l}\text { Mean Difference (IV, Random, 95\% } \\
\mathrm{CI})\end{array}$ & $0.59[-1.46,2.63]$ \\
\hline 1.4.1 HDRS (high score = more depressed) & 4 & 100 & $\begin{array}{l}\text { Mean Difference (IV, Random, 95\% } \\
\mathrm{CI})\end{array}$ & $0.59[-1.46,2.63]$ \\
\hline $\begin{array}{l}1.5 \text { Cognition: average change in scores be- } \\
\text { tween baseline and end of treatment }\end{array}$ & 3 & 159 & $\begin{array}{l}\text { Mean Difference (IV, Random, 95\% } \\
\mathrm{CI})\end{array}$ & $0.11[-1.02,1.24]$ \\
\hline $\begin{array}{l}\text { 1.5.1 MMSE (low score = cognitive impair- } \\
\text { ment) }\end{array}$ & 3 & 159 & $\begin{array}{l}\text { Mean Difference (IV, Random, 95\% } \\
\mathrm{CI})\end{array}$ & $0.11[-1.02,1.24]$ \\
\hline $\begin{array}{l}\text { 1.6 Cognition: Mean scores at end of treat- } \\
\text { ment }\end{array}$ & 2 & 48 & $\begin{array}{l}\text { Mean Difference (IV, Random, 95\% } \\
\mathrm{CI})\end{array}$ & $-0.42[-2.60,1.76]$ \\
\hline $\begin{array}{l}\text { 1.6.1 MMSE (low score = cognitive impair- } \\
\text { ment) }\end{array}$ & 2 & 48 & $\begin{array}{l}\text { Mean Difference (IV, Random, 95\% } \\
\mathrm{CI})\end{array}$ & $-0.42[-2.60,1.76]$ \\
\hline $\begin{array}{l}\text { 1.7 Activities of daily living: average change } \\
\text { in scores between baseline and end of treat- } \\
\text { ment }\end{array}$ & 3 & 57 & $\begin{array}{l}\text { Mean Difference (IV, Random, 95\% } \\
\mathrm{CI})\end{array}$ & $\begin{array}{l}1.18[-7.77 \\
10.14]\end{array}$ \\
\hline $\begin{array}{l}\text { 1.7.1 Barthel Index (high score = more depen- } \\
\text { dent) }\end{array}$ & 3 & 57 & $\begin{array}{l}\text { Mean Difference (IV, Random, 95\% } \\
\mathrm{CI})\end{array}$ & $\begin{array}{l}1.18[-7.77 \\
10.14]\end{array}$ \\
\hline $\begin{array}{l}\text { 1.8 Activities of daily living: mean scores at } \\
\text { end of treatment }\end{array}$ & 3 & 116 & $\begin{array}{l}\text { Mean Difference (IV, Random, 95\% } \\
\mathrm{CI})\end{array}$ & $-3.86[-9.48,1.77]$ \\
\hline $\begin{array}{l}\text { 1.8.1 Barthel Index (high score = more depen- } \\
\text { dent) }\end{array}$ & 2 & 52 & $\begin{array}{l}\text { Mean Difference (IV, Random, 95\% } \\
\mathrm{CI})\end{array}$ & $\begin{array}{l}0.75[-12.97 \\
14.47]\end{array}$ \\
\hline $\begin{array}{l}\text { 1.8.2 Activities of daily living Index (high score } \\
=\text { more dependent) }\end{array}$ & 1 & 64 & $\begin{array}{l}\text { Mean Difference (IV, Random, 95\% } \\
\mathrm{CI})\end{array}$ & $\begin{array}{l}-5.18[-7.38 \\
-2.98]\end{array}$ \\
\hline $\begin{array}{l}\text { 1.9 Disability: average change in scores be- } \\
\text { tween baseline and end of treatment }\end{array}$ & 4 & & $\begin{array}{l}\text { Mean Difference (IV, Random, 95\% } \\
\mathrm{CI})\end{array}$ & $\begin{array}{l}\text { Totals not select- } \\
\text { ed }\end{array}$ \\
\hline
\end{tabular}




\begin{tabular}{|c|c|c|c|c|}
\hline Outcome or subgroup title & $\begin{array}{l}\text { No. of } \\
\text { studies }\end{array}$ & $\begin{array}{l}\text { No. of } \\
\text { partici- } \\
\text { pants }\end{array}$ & Statistical method & Effect size \\
\hline $\begin{array}{l}\text { 1.9.1 Hemispheric Stroke Scale Total Score } \\
\text { (high score = more neurological deficit) }\end{array}$ & 2 & & $\begin{array}{l}\text { Mean Difference (IV, Random, 95\% } \\
\mathrm{CI})\end{array}$ & $\begin{array}{l}\text { Totals not select- } \\
\text { ed }\end{array}$ \\
\hline $\begin{array}{l}\text { 1.9.2 Hemispheric Stroke Scale Motor Sub- } \\
\text { scale (high score }=\text { more deficit) }\end{array}$ & 2 & & $\begin{array}{l}\text { Mean Difference (IV, Random, 95\% } \\
\mathrm{Cl} \text { ) }\end{array}$ & $\begin{array}{l}\text { Totals not select- } \\
\text { ed }\end{array}$ \\
\hline $\begin{array}{l}\text { 1.9.3 Hemispheric Stroke Scale Gait Subscale } \\
\text { (high score = more deficit) }\end{array}$ & 2 & & $\begin{array}{l}\text { Mean Difference (IV, Random, 95\% } \\
\mathrm{CI})\end{array}$ & $\begin{array}{l}\text { Totals not select- } \\
\text { ed }\end{array}$ \\
\hline $\begin{array}{l}\text { 1.9.4 Johns Hopkins Functioning Inventory } \\
\text { (high score = less function) }\end{array}$ & 2 & & $\begin{array}{l}\text { Mean Difference (IV, Random, 95\% } \\
\mathrm{CI})\end{array}$ & $\begin{array}{l}\text { Totals not select- } \\
\text { ed }\end{array}$ \\
\hline $\begin{array}{l}\text { 1.10 Disability: mean scores at end of treat- } \\
\text { ment }\end{array}$ & 4 & & $\begin{array}{l}\text { Mean Difference (IV, Random, 95\% } \\
\mathrm{CI})\end{array}$ & $\begin{array}{l}\text { Totals not select- } \\
\text { ed }\end{array}$ \\
\hline $\begin{array}{l}\text { 1.10.1 Hemispheric Stroke Scale total score } \\
\text { (high score = more neurological deficit) }\end{array}$ & 2 & & $\begin{array}{l}\text { Mean Difference (IV, Random, 95\% } \\
\mathrm{CI})\end{array}$ & $\begin{array}{l}\text { Totals not select- } \\
\text { ed }\end{array}$ \\
\hline $\begin{array}{l}\text { 1.10.2 Hemispheric Stroke Scale motor sub- } \\
\text { scale (high score = more deficit) }\end{array}$ & 2 & & $\begin{array}{l}\text { Mean Difference (IV, Random, 95\% } \\
\mathrm{CI})\end{array}$ & $\begin{array}{l}\text { Totals not select- } \\
\text { ed }\end{array}$ \\
\hline $\begin{array}{l}\text { 1.10.3 Hemispheric Stroke Scale gait subscale } \\
\text { (high score = more deficit) }\end{array}$ & 2 & & $\begin{array}{l}\text { Mean Difference (IV, Random, 95\% } \\
\mathrm{CI})\end{array}$ & $\begin{array}{l}\text { Totals not select- } \\
\text { ed }\end{array}$ \\
\hline $\begin{array}{l}\text { 1.10.4 Johns Hopkins Functioning Inventory } \\
\text { (high score = less function) }\end{array}$ & 2 & & $\begin{array}{l}\text { Mean Difference (IV, Random, 95\% } \\
\mathrm{CI})\end{array}$ & $\begin{array}{l}\text { Totals not select- } \\
\text { ed }\end{array}$ \\
\hline $\begin{array}{l}\text { 1.11 Disability: moderate to severe disability } \\
\text { at end of treatment }\end{array}$ & 1 & 111 & Risk Ratio (M-H, Fixed, 95\% Cl) & Not estimable \\
\hline 1.11.1 Modified Rankin Scale & 1 & 111 & Risk Ratio (M-H, Fixed, 95\% Cl) & Not estimable \\
\hline 1.12 Adverse events: death & 9 & 496 & Risk Ratio (M-H, Random, 95\% Cl) & $1.25[0.32,4.91]$ \\
\hline 1.12.1 At end of treatment & 9 & 496 & Risk Ratio (M-H, Random, 95\% Cl) & $1.25[0.32,4.91]$ \\
\hline 1.13 Adverse events: all & 9 & & Risk Ratio (M-H, Random, 95\% Cl) & Subtotals only \\
\hline $\begin{array}{l}\text { 1.13.1 Central nervous system events (e.g. } \\
\text { confusion, sedation, tremor) }\end{array}$ & 4 & 214 & Risk Ratio (M-H, Random, 95\% Cl) & $1.26[0.51,3.15]$ \\
\hline $\begin{array}{l}\text { 1.13.2 Gastrointestinal effects (e.g. constipa- } \\
\text { tion, diarrhoea) }\end{array}$ & 6 & 383 & Risk Ratio (M-H, Random, 95\% Cl) & $1.21[0.67,2.19]$ \\
\hline 1.13.3 Recurrent stroke & 2 & 114 & Risk Ratio $(\mathrm{M}-\mathrm{H}$, Random, 95\% Cl) & $1.49[0.25,8.80]$ \\
\hline $\begin{array}{l}\text { 1.13.4 Vascular events - not stroke (e.g. dizzi- } \\
\text { ness, palpitation) }\end{array}$ & 3 & 282 & Risk Ratio (M-H, Random, 95\% Cl) & $1.13[0.84,1.50]$ \\
\hline $\begin{array}{l}\text { 1.13.5 Psychiatric events (e.g. anxiety, in- } \\
\text { creased depression) }\end{array}$ & 1 & 100 & Risk Ratio (M-H, Random, 95\% Cl) & $4.81[0.24,97.68]$ \\
\hline $\begin{array}{l}\text { 1.13.6 Other events - not listed above (e.g. dy- } \\
\text { suria, eye discomfort) }\end{array}$ & 3 & 190 & Risk Ratio (M-H, Random, 95\% Cl) & $1.12[0.44,2.85]$ \\
\hline
\end{tabular}




\begin{tabular}{llllll}
\hline Outcome or subgroup title & No. of & & $\begin{array}{l}\text { No. of } \\
\text { partici- } \\
\text { pants }\end{array}$ & Statistical method & Effect size \\
\hline $\begin{array}{l}1.13 .7 \text { Protocol violation (e.g. refused treat- } \\
\text { ment, withdrew consent) }\end{array}$ & 5 & 309 & Risk Ratio (M-H, Random, 95\% Cl) & $0.89[0.62,1.27]$ \\
\hline $\begin{array}{l}1.14 \text { Adverse events: leaving the study early } \\
\text { (including death) }\end{array}$ & 12 & 836 & Risk Ratio (M-H, Random, 95\% Cl) & $1.03[0.84,1.26]$ \\
\hline \begin{tabular}{l}
1.14 .1 All dropouts and withdrawals \\
\hline
\end{tabular} & 12 & 836 & Risk Ratio (M-H, Random, 95\% Cl) & $1.03[0.84,1.26]$ \\
\hline
\end{tabular}




\section{Analysis 1.1. Comparison 1: Pharmacological interventions (antidepressants) versus placebo, Outcome 1: Depression: meeting study criteria for depression at end treatment}

\begin{tabular}{|c|c|c|c|c|c|c|c|}
\hline \multirow[b]{2}{*}{ Study or Subgroup } & \multicolumn{3}{|c|}{ Favours pharmacotherapy } & \multicolumn{2}{|c|}{ Placebo } & \multirow[b]{2}{*}{ Weight } & \multirow{2}{*}{$\begin{array}{c}\text { Risk Ratio } \\
\text { M-H, Random, 95\% CI }\end{array}$} \\
\hline & Events & & Total & Events & Total & & \\
\hline \multicolumn{8}{|c|}{ 1.1.1 CGI (high score = more severe) } \\
\hline Rasmussen 2003 & & 13 & 70 & 20 & 67 & $24.7 \%$ & $0.62[0.34,1.15]$ \\
\hline Subtotal (95\% CI) & & & 70 & & 67 & $24.7 \%$ & $0.62[0.34,1.15]$ \\
\hline Total events: & & 13 & & 20 & & & \\
\hline
\end{tabular}

Total events:

Heterogeneity: Not applicable

Test for overall effect: $Z=1.52(P=0.13)$

1.1.2 Clinician interview/impression (no improvement)

$\begin{array}{lll}\text { Roh } 1996 & 10 & 32 \\ \text { Subtotal }(\mathbf{9 5 \%} \text { CI) } & & \mathbf{3 2}\end{array}$

Total events: $\quad 10$

Heterogeneity: Not applicable

Test for overall effect: $\mathrm{Z}=2.80(\mathrm{P}=0.005)$

\subsubsection{Meets DSM-III-R criteria}

Palomaki 1999

Robinson 2000a

Robinson 2000b

Subtotal $(95 \%$ CI)

Total events:

$\begin{array}{ll}5 & 5 \\ 3 & 17 \\ 1 & 15 \\ & \mathbf{8}\end{array}$

51
17
15
83

$\begin{array}{rrr}5 & 49 & 6.7 \% \\ 3 & 8 & 5.0 \% \\ 2 & 8 & 1.8 \% \\ & \mathbf{6 5} & \mathbf{1 3 . 5 \%}\end{array}$

Heterogeneity: $\mathrm{Tau}^{2}=0.00 ; \mathrm{Chi}^{2}=1.24, \mathrm{df}=2(\mathrm{P}=0.54) ; \mathrm{I}^{2}=0 \%$

Test for overall effect: $\mathrm{Z}=1.13(\mathrm{P}=0.26)$

1.1.4 Meets DSM-IV criteria

$\begin{array}{lllllll}\text { Robinson 2008 } & 5 & 58 & 13 & 59 & 9.9 \% & 0.39[0.15,1.03] \\ \text { Xu 2006 } & 3 & 32 & 12 & 32 & 6.8 \% & 0.25[0.08,0.80] \\ \text { Subtotal (95\% CI) } & & \mathbf{9 0} & & \mathbf{9 1} & \mathbf{1 6 . 7 \%} & \mathbf{0 . 3 3}[\mathbf{0 . 1 5 , 0 . 6 9 ]}\end{array}$

Total events:

8

Heterogeneity: $\mathrm{Tau}^{2}=0.00 ; \mathrm{Chi}^{2}=0.34, \mathrm{df}=1(\mathrm{P}=0.56) ; \mathrm{I}^{2}=0 \%$

Test for overall effect: $\mathrm{Z}=2.95(\mathrm{P}=0.003)$

1.1.5 HADS (high score $=$ more severe $)$

Almeida $2006 \quad 8$

Subtotal (95\% CI)

$\begin{array}{llll}55 & 11 & 56 & 13.4 \% \\ \mathbf{5 5} & & \mathbf{5 6} & \mathbf{1 3 . 4 \%}\end{array}$

$0.74[0.32,1.70]$

$0.74[0.32,1.70]$

11

Heterogeneity: Not applicable

Test for overall effect: $\mathrm{Z}=0.71(\mathrm{P}=0.48)$

1.1.6 HDRS $($ high score $=$ more depressed)

Tsai 2011

Subtotal (95\% CI)

1

Total events:

Heterogeneity: Not applicable

Test for overall effect: $\mathrm{Z}=1.86(\mathrm{P}=0.06)$

Total $(95 \%$ CI $)$

$358 \quad 100.0 \%$

Total events:

$49 \quad 96$

Heterogeneity: $\mathrm{Tau}^{2}=0.00 ; \mathrm{Chi}^{2}=6.06, \mathrm{df}=8(\mathrm{P}=0.64) ; \mathrm{I}^{2}=0 \%$

Test for overall effect: $\mathrm{Z}=4.43(\mathrm{P}<0.00001)$

Test for subgroup differences: $\mathrm{Chi}^{2}=4.45, \mathrm{df}=5(\mathrm{P}=0.49), \mathrm{I}^{2}=0 \%$
$0.96[0.30,3.11]$

$0.47[0.12,1.84]$

$0.27[0.03,2.51]$

$0.62[0.27,1.42]$

Risk Ratio

M-H, Random, 95\% CI 
Analysis 1.5. Comparison 1: Pharmacological interventions (antidepressants) versus placebo, Outcome 5: Cognition: average change in scores between baseline and end of treatment

\begin{tabular}{|c|c|c|c|c|c|c|c|c|c|}
\hline & \multicolumn{3}{|c|}{ Pharmacotherapy } & \multicolumn{3}{|c|}{ Placebo } & \multicolumn{2}{|r|}{ Mean Difference } & Mean Difference \\
\hline Study or Subgroup & Mean & SD & Total & Mean & SD & Total & Weight & IV, Random, $95 \%$ CI & IV, Random, $95 \%$ CI \\
\hline
\end{tabular}

1.5.1 MMSE (low score = cognitive impairment)

Almeida 2006

Robinson 2000

Robinson 2000b

$\begin{array}{ll}2.6 & 3\end{array}$

$0.2 \quad 3.75$

55
17

$55 \quad 2.6$

$\begin{array}{ll}2.6 & 3.9\end{array}$

3.96

$\begin{array}{rr}56 & 76.3 \% \\ 8 & 11.9 \%\end{array}$

Subtotal (95\% CI)

$0.3 \quad 3.58$

15
87

$-0.2 \quad 3.96$

$8 \quad 11.8 \%$

$72 \quad \mathbf{1 0 0 . 0 \%}$

$0.50[-2.79,3.79]$

Heterogeneity: $\mathrm{Tau}^{2}=0.00 ; \mathrm{Chi}^{2}=0.11, \mathrm{df}=2(\mathrm{P}=0.95) ; \mathrm{I}^{2}=0 \%$

Test for overall effect: $\mathrm{Z}=0.19(\mathrm{P}=0.85)$

Total $(95 \%$ CI)

87

Heterogeneity: $\mathrm{Tau}^{2}=0.00 ; \mathrm{Chi}^{2}=0.11, \mathrm{df}=2(\mathrm{P}=0.95) ; \mathrm{I}^{2}=0 \%$

Test for overall effect: $\mathrm{Z}=0.19(\mathrm{P}=0.85)$

Test for subgroup differences: Not applicable

$72 \quad 100.0 \%$

$0.11[-1.02,1.24]$

, Random, 95\% CI

\section{Analysis 1.6. Comparison 1: Pharmacological interventions (antidepressants) versus placebo, Outcome 6: Cognition: Mean scores at end of treatment}

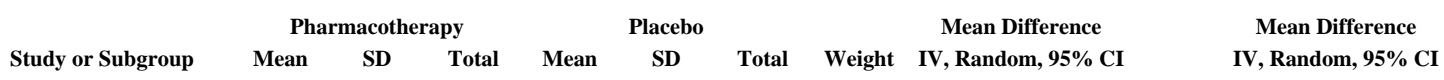

\begin{tabular}{lllllllll}
\hline 1.6.1 MMSE (low score & cognitive & impairment) & & & & & & \\
Robinson 2000a & 26.1 & 7.5 & 17 & 26.8 & 2.4 & 8 & $30.7 \%$ & $-0.70[-4.63,3.23]$ \\
Robinson 2000b & 26.5 & 4 & 15 & 26.8 & 2.4 & 8 & $69.3 \%$ & $-0.30[-2.92,2.32]$
\end{tabular}

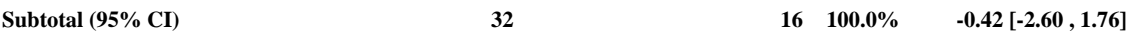

Heterogeneity: $\mathrm{Tau}^{2}=0.00 ; \mathrm{Chi}^{2}=0.03, \mathrm{df}=1(\mathrm{P}=0.87) ; \mathrm{I}^{2}=0 \%$

Test for overall effect: $\mathrm{Z}=0.38(\mathrm{P}=0.70)$

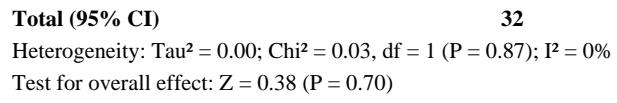

Heterogeneity: $\mathrm{Tau}^{2}=0.00 ; \mathrm{Chi}^{2}=0.03, \mathrm{df}=1(\mathrm{P}=0.87) ; \mathrm{I}^{2}=0 \%$

Test for overall effect: $\mathrm{Z}=0.38(\mathrm{P}=0.70)$

Test for subgroup differences: Not applicable

$16 \quad 100.0 \% \quad-0.42[-2.60,1.76]$

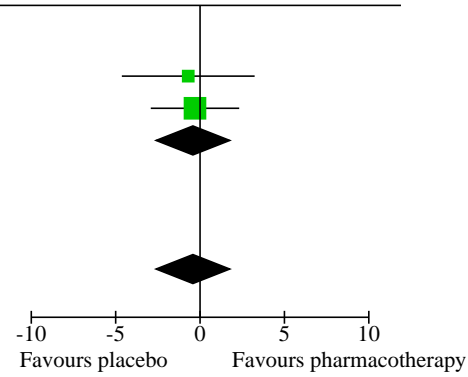

Analysis 1.7. Comparison 1: Pharmacological interventions (antidepressants) versus placebo, Outcome 7: Activities of daily living: average change in scores between baseline and end of treatment

\begin{tabular}{|c|c|c|c|c|c|c|c|c|c|}
\hline & \multicolumn{3}{|c|}{ Pharmacotherapy } & \multicolumn{3}{|c|}{ Placebo } & \multirow{2}{*}{\multicolumn{2}{|c|}{$\begin{array}{c}\text { Mean Difference } \\
\text { IV, Random, 95\% CI }\end{array}$}} & \multirow{2}{*}{$\begin{array}{c}\text { Mean Difference } \\
\text { IV, Random, 95\% CI }\end{array}$} \\
\hline Study or Subgroup & Mean & SD & Total & Mean & SD & Total & & & \\
\hline
\end{tabular}

1.7.1 Barthel Index (high score = more dependent)

$\begin{array}{lrrrrrrrr}\text { Dam 1996a } & -23.5 & 12.13 & 18 & -19 & 16.54 & 8 & 49.3 \% & -4.50[-17.26,8.26] \\ \text { Dam 1996b } & -11.5 & 16.69 & 17 & -19.1 & 16.76 & 9 & 43.9 \% & 7.60[-5.92,21.12] \\ \text { Reding 1986 } & -24 & 27.71 & 3 & -25 & 9.9 & 2 & 6.8 \% & 1.00[-33.23,35.23] \\ \text { Subtotal (95\% CI) } & & & \mathbf{3 8} & & & \mathbf{1 9} & \mathbf{1 0 0 . 0 \%} & \mathbf{1 . 1 8}[-\mathbf{- 7 . 7 7}, \mathbf{1 0 . 1 4}]\end{array}$

Heterogeneity: $\mathrm{Tau}^{2}=0.00 ; \mathrm{Chi}^{2}=1.63, \mathrm{df}=2(\mathrm{P}=0.44) ; \mathrm{I}^{2}=0 \%$

Test for overall effect: $\mathrm{Z}=0.26(\mathrm{P}=0.80)$

Total $(95 \%$ CI $)$

38

Heterogeneity: $\mathrm{Tau}^{2}=0.00 ; \mathrm{Chi}^{2}=1.63, \mathrm{df}=2(\mathrm{P}=0.44) ; \mathrm{I}^{2}=0 \%$

Test for overall effect: $\mathrm{Z}=0.26(\mathrm{P}=0.80)$

Test for subgroup differences: Not applicable

$19 \quad 100.0 \% \quad 1.18[-7.77,10.14]$

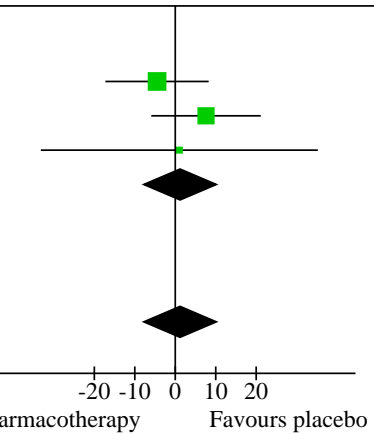


Analysis 1.8. Comparison 1: Pharmacological interventions (antidepressants) versus placebo, Outcome 8: Activities of daily living: mean scores at end of treatment

\begin{tabular}{|c|c|c|c|c|c|c|c|c|c|c|c|}
\hline \multirow[b]{2}{*}{ Study or Subgroup } & \multicolumn{3}{|c|}{ Pharmacotherapy } & \multicolumn{3}{|c|}{ Placebo } & \multirow[b]{2}{*}{ Weight } & \multirow{2}{*}{$\begin{array}{c}\text { Mean Difference } \\
\text { IV, Random, 95\% CI }\end{array}$} & \multirow{2}{*}{\multicolumn{2}{|c|}{$\begin{array}{c}\text { Mean Difference } \\
\text { IV, Random, 95\% CI }\end{array}$}} & \\
\hline & Mean & SD & Total & Mean & SD & Total & & & & & \\
\hline \multicolumn{12}{|c|}{ 1.8.1 Barthel Index (high score = more dependent) } \\
\hline Dam 1996a & 61.9 & 13 & 18 & 54.1 & 21.1 & 8 & $11.1 \%$ & $7.80[-8.01,23.61]$ & & & \\
\hline Dam 1996b & 47.9 & 15.5 & 17 & 54.1 & 21.1 & 9 & $11.3 \%$ & $-6.20[-21.83,9.43]$ & & - & \\
\hline Subtotal $(95 \%$ CI $)$ & & & 35 & & & 17 & $22.4 \%$ & $0.75[-12.97,14.47]$ & & & \\
\hline \multicolumn{12}{|c|}{ Heterogeneity: $\mathrm{Tau}^{2}=33.68 ; \mathrm{Chi}^{2}=1.52, \mathrm{df}=1(\mathrm{P}=0.22) ; \mathrm{I}^{2}=34 \%$} \\
\hline \multicolumn{12}{|c|}{ Test for overall effect: $\mathrm{Z}=0.11(\mathrm{P}=0.91)$} \\
\hline \multicolumn{12}{|c|}{ 1.8.2 Activities of daily living Index (high score = more dependent) } \\
\hline Xu 2006 & 27.63 & 4.81 & 32 & 32.81 & 4.13 & 32 & $77.6 \%$ & $-5.18[-7.38,-2.98]$ & & & \\
\hline Subtotal $(95 \%$ CI $)$ & & & 32 & & & 32 & $\mathbf{7 7 . 6 \%}$ & $-5.18[-7.38,-2.98]$ & & & \\
\hline \multicolumn{12}{|c|}{ Heterogeneity: Not applicable } \\
\hline \multicolumn{12}{|c|}{ Test for overall effect: $\mathrm{Z}=4.62(\mathrm{P}<0.00001)$} \\
\hline Total $(95 \%$ CI $)$ & & & 67 & & & 49 & $100.0 \%$ & $-3.86[-9.48,1.77]$ & & & \\
\hline \multicolumn{12}{|c|}{ Heterogeneity: $\mathrm{Tau}^{2}=9.36 ; \mathrm{Chi}^{2}=2.57, \mathrm{df}=2(\mathrm{P}=0.28) ; \mathrm{I}^{2}=22 \%$} \\
\hline \multicolumn{9}{|c|}{ Test for overall effect: $\mathrm{Z}=1.34(\mathrm{P}=0.18)$} & $-20-10$ & 10 & 20 \\
\hline \multicolumn{11}{|c|}{ Test for subgroup differences: $\mathrm{Chi}^{2}=0.70, \mathrm{df}=1(\mathrm{P}=0.40), \mathrm{I}^{2}=0 \%$} & s placebo \\
\hline
\end{tabular}

\section{Analysis 1.9. Comparison 1: Pharmacological interventions (antidepressants) versus placebo, Outcome 9: Disability: average change in scores between baseline and end of treatment}

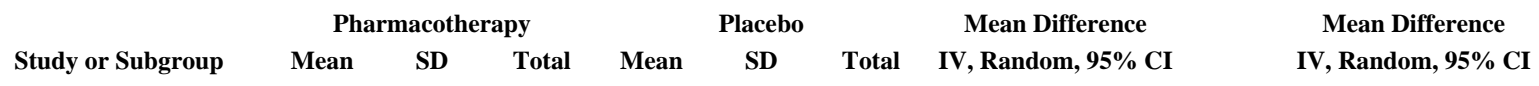

1.9.1 Hemispheric Stroke Scale Total Score (high score = more neurological deficit)

$\begin{array}{lllllll}\text { Dam 1996a } & -5.3 & 9.6 & 18 & -5 & 9.9 & 8\end{array}$

Dam 1996b

$-6.1 \quad 13.26$

17

9.9

9

$-0.30[-8.47,7.87]$

$-1.10[-10.13,7.93]$

1.9.2 Hemispheric Stroke Scale Motor Subscale (high score = more deficit)

\begin{tabular}{|c|c|c|c|c|c|c|c|}
\hline Dam 1996a & -3 & 3.85 & 18 & -3.9 & 4.9 & 8 & $0.90[-2.93,4.73]$ \\
\hline Dam 1996b & -3.2 & 4.75 & 17 & -3.9 & 4.9 & 9 & $0.70[-3.22,4.62]$ \\
\hline
\end{tabular}

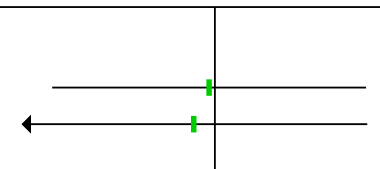

Dam 1996b

$-3.2 \quad 4.75$

$17-3.9$

$0.70[-3.22,4.62]$

1.9.3 Hemispheric Stroke Scale Gait Subscale (high score = more deficit)

$\begin{array}{llllll}\text { Dam 1996a } & -2.1 & 0.13 & 18 & -1.2 & 0.96\end{array}$

Dam 1996b

$\begin{array}{lllll}-0.9 & 1.17 & 17 & -1.2 & 0.96\end{array}$

$-0.90[-1.57,-0.23]$

$0.30[-0.54,1.14]$

1.9.4 Johns Hopkins Functioning Inventory (high score = less function)

$\begin{array}{llllll}\text { Robinson 2000a } & 0.7 & 3.82 & 17 & 2 & 3.93\end{array}$

$\begin{array}{llllll}\text { Robinson 2000b } & 1.5 & 3.14 & 15 & 2 & 3.93\end{array}$

$-1.30[-4.57,1.97]$

$-0.50[-3.65,2.65]$

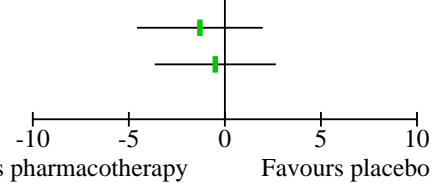


Analysis 1.10. Comparison 1: Pharmacological interventions (antidepressants) versus placebo, Outcome 10: Disability: mean scores at end of treatment

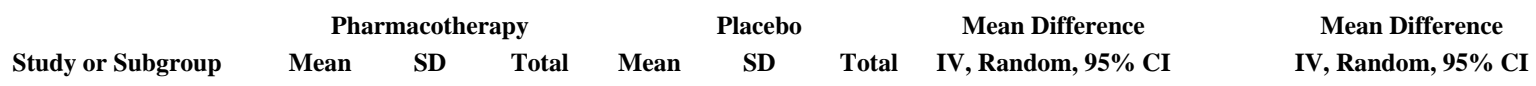

1.10.1 Hemispheric Stroke Scale total score (high score = more neurological deficit)

$\begin{array}{lllllll}\text { Dam 1996a } & 44.1 & 9.4 & 18 & 46.8 & 9.9 & 8\end{array}$

Dam 1996b

48.3

13.8

$17 \quad 46.8$

9.9

$-2.70[-10.82,5.42]$

$1.50[-7.71,10.71]$

1.10.2 Hemispheric Stroke Scale motor subscale (high score = more deficit)

$\begin{array}{llllllll}\text { Dam 1996a } & 32.4 & 3.8 & 18 & 31.6 & 5 & 8 & 0.80[-3.08,4.68] \\ \text { Dam 1996b } & 32.2 & 4.7 & 17 & 31.6 & 5 & 9 & 0.60[-3.36,4.56]\end{array}$

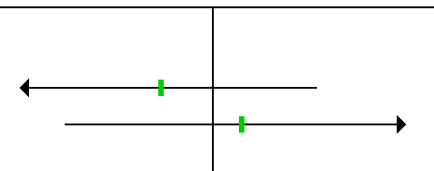

Dam 1996b

$32.2 \quad 4.7$

$17 \quad 31.6$

\begin{tabular}{lllll} 
1.10.3 Hemispheric Stroke Scale gait subscale (high score & \multicolumn{3}{c}{ more deficit) } \\
Dam $1996 \mathrm{a}$ & 3.8 & 0.9 & 18 & 4.6
\end{tabular}

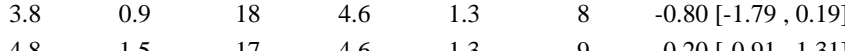

1.10.4 Johns Hopkins Functioning Inventory (high score = less function)

$\begin{array}{llllll}\text { Robinson 2000a } & 3.8 & 3.4 & 17 & 2.9 & 3.4\end{array}$

$\begin{array}{llllll}\text { Robinson } 2000 \mathrm{~b} & 2.4 & 2.3 & 15 & 2.9 & 3.4\end{array}$

$0.90[-1.96,3.76]$

$-0.50[-3.13,2.13]$

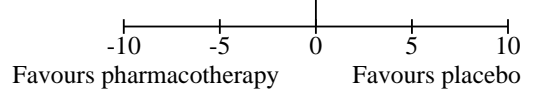

\section{Analysis 1.11. Comparison 1: Pharmacological interventions (antidepressants) versus placebo, Outcome 11: Disability: moderate to severe disability at end of treatment}

\begin{tabular}{|c|c|c|c|c|c|c|c|}
\hline & Pharm & herapy & $\mathbf{P l}$ & & & Risk Ratio & Risk Ratio \\
\hline Study or Subgroup & Events & Total & Events & Total & Weight & M-H, Fixed, 95\% CI & M-H, Fixed, $95 \%$ CI \\
\hline
\end{tabular}

\subsubsection{Modified Rankin Scale}

Almeida 2006

Subtotal (95\% CI)

0

Total events: 0

Heterogeneity: Not applicable

Test for overall effect: Not applicable

\section{Total $(95 \%$ CI $)$}

55

Total events:

0

55
55

55

56

56

0

Heterogeneity: Not applicable

Test for overall effect: Not applicable

Test for subgroup differences: Not applicable

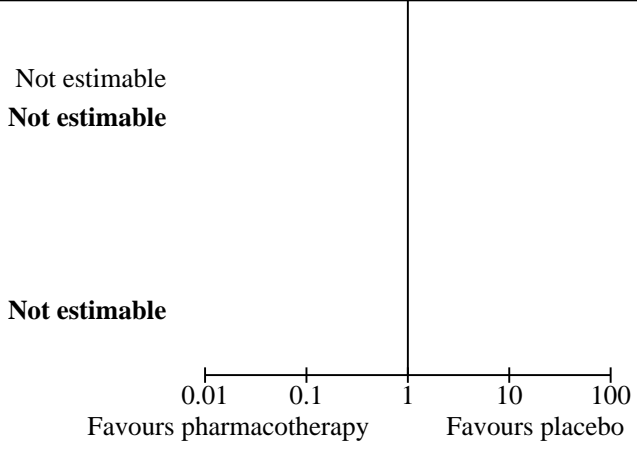




\section{Analysis 1.12. Comparison 1: Pharmacological interventions} (antidepressants) versus placebo, Outcome 12: Adverse events: death

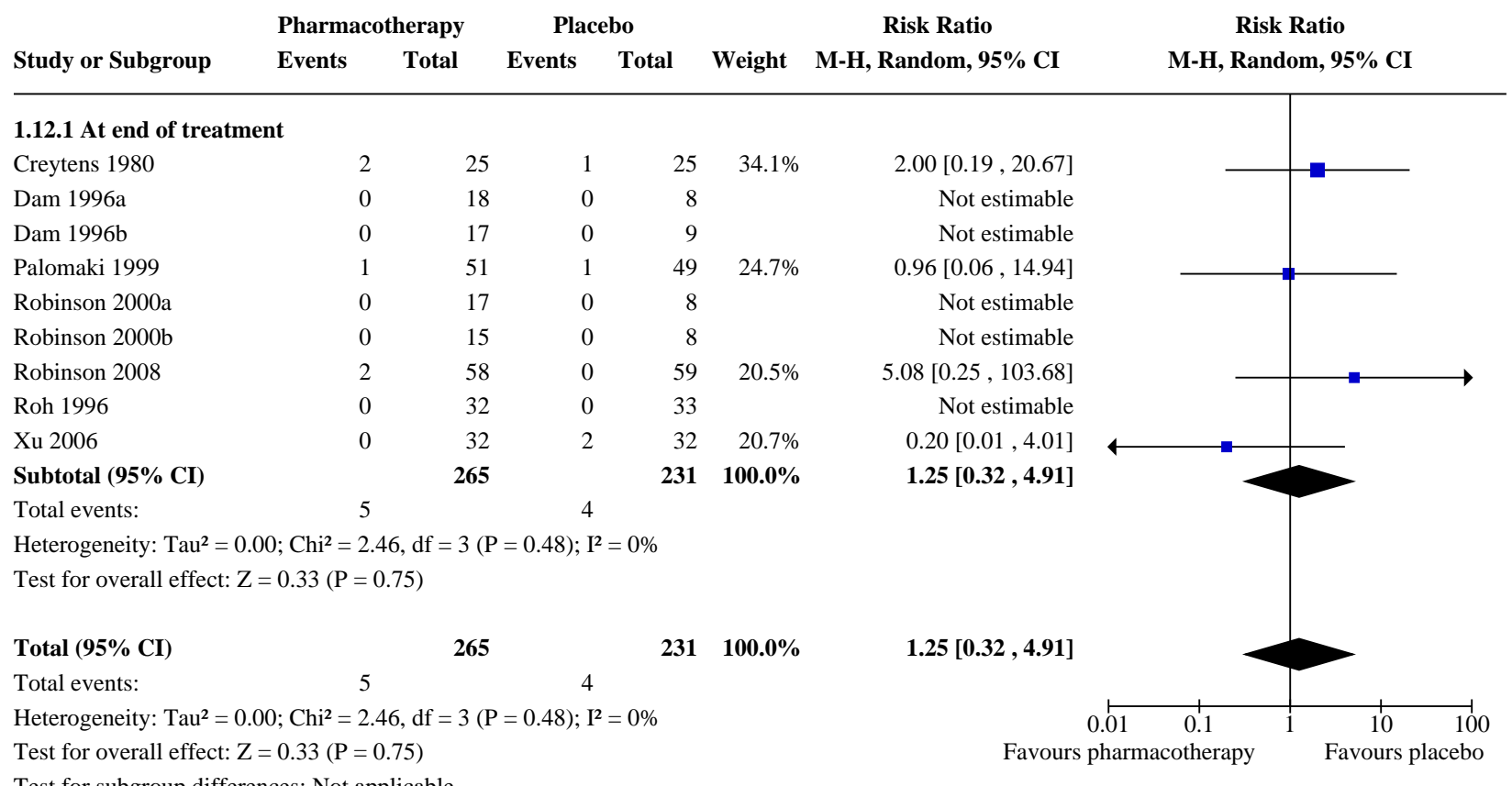


Analysis 1.13. Comparison 1: Pharmacological interventions (antidepressants) versus placebo, Outcome 13: Adverse events: all

\begin{tabular}{|c|c|c|c|c|c|c|c|}
\hline \multirow{2}{*}{ tudy or Subgroup } & \multicolumn{2}{|c|}{ Pharmacotherapy } & \multicolumn{2}{|c|}{ Placebo } & \multicolumn{2}{|r|}{ Risk Ratio } & \multirow{2}{*}{$\begin{array}{c}\text { Risk Ratio } \\
\text { M-H, Random, 95\% CI }\end{array}$} \\
\hline & Events & Total & Events & Total & Weight & M-H, Random, 95\% CI & \\
\hline
\end{tabular}

1.13.1 Central nervous system events (e.g. confusion, sedation, tremor)

Dam 1996a

Palomaki 1999

Robinson 2000b

Roh 1996

Subtotal (95\% CI)

Total events:

Heterogeneity: $\mathrm{Tau}^{2}=0.00 ; \mathrm{Chi}^{2}=1.90, \mathrm{df}=3(\mathrm{P}=0.59) ; \mathrm{I}^{2}=0 \%$

Test for overall effect: $\mathrm{Z}=0.50(\mathrm{P}=0.62)$

1.13.2 Gastrointestinal effects (e.g. constipation, diarrhoea)

$\begin{array}{lrrrrr}\text { Creytens 1980 } & 1 & 25 & 0 & 25 & 3.5 \% \\ \text { Dam 1996a } & 1 & 18 & 0 & 8 & 3.6 \% \\ \text { Palomaki 1999 } & 2 & 51 & 1 & 49 & 6.2 \% \\ \text { Robinson 2000a } & 1 & 17 & 0 & 8 & 3.6 \% \\ \text { Robinson 2008 } & 14 & 59 & 13 & 58 & 79.5 \% \\ \text { Roh 1996 } & 1 & 32 & 0 & 33 & 3.5 \% \\ \text { Subtotal (95\% CI) } & & \mathbf{2 0 2} & & \mathbf{1 8 1} & \mathbf{1 0 0 . 0 \%} \\ \text { Total events: } & 20 & & 14 & & \end{array}$

Total events:

20

Heterogeneity: $\mathrm{Tau}^{2}=0.00 ; \mathrm{Chi}^{2}=1.01, \mathrm{df}=5(\mathrm{P}=0.96) ; \mathrm{I}^{2}=0 \%$

Test for overall effect: $\mathrm{Z}=0.63(\mathrm{P}=0.53)$

\subsubsection{Recurrent stroke}

Creytens 1980

Xu 2006

Subtotal (95\% CI)

Total events:

2

25

25
32

57

3

2

Heterogeneity: $\mathrm{Tau}^{2}=0.00 ; \mathrm{Chi}^{2}=0.14, \mathrm{df}=1(\mathrm{P}=0.71) ; \mathrm{I}^{2}=0 \%$

Test for overall effect: $\mathrm{Z}=0.44(\mathrm{P}=0.66)$

1.13.4 Vascular events - not stroke (e.g. dizziness, palpitation)

Robinson 2008

Roh 1996

Subtotal (95\% CI)

$\begin{array}{rr}1 & 51 \\ 37 & 58 \\ 1 & 32 \\ & \mathbf{1 4 1}\end{array}$

0
34
0

$49 \quad 0.8 \%$

$98.3 \%$

Total events:

39

34

Heterogeneity: $\mathrm{Tau}^{2}=0.00 ; \mathrm{Chi}^{2}=0.79, \mathrm{df}=2(\mathrm{P}=0.68) ; \mathrm{I}^{2}=0 \%$

Test for overall effect: $\mathrm{Z}=0.80(\mathrm{P}=0.42)$

1.13.5 Psychiatric events (e.g. anxiety, increased depression)

$\begin{array}{lllllll}\text { Palomaki } 1999 & 2 & 51 & 0 & 49 & 100.0 \% & 4.81[0.24,97.68] \\ \begin{array}{l}\text { Subtotal }(\mathbf{9 5 \%} \text { CI) } \\ \text { Total events: }\end{array} & 2 & \mathbf{5 1} & & \mathbf{4 9} & \mathbf{1 0 0 . 0 \%} & \mathbf{4 . 8 1}[\mathbf{0 . 2 4}, \mathbf{9 7 . 6 8}] \\ \end{array}$

Heterogeneity: Not applicable

Test for overall effect: $\mathrm{Z}=1.02(\mathrm{P}=0.31)$

1.13.6 Other events - not listed above (e.g. dysuria, eye discomfort)

$\begin{array}{lllll}\text { Palomaki } 1999 & 7 & 51 & 5 & 49\end{array}$

Robinson 2000a

Roh 1996

Subtotal (95\% CI)

$2 \quad 17$

17

1

$74.3 \%$

$17.1 \%$

$1.35[0.46,3.96]$

$0.94[0.10,8.92]$

$0.34[0.01,8.13]$

$1.12[0.44,2.85]$

$2.88[0.12,69.16]$

$1.11[0.83,1.48]$

$3.09[0.13,73.19]$

$1.13[0.84,1.50]$

100

$100.0 \%$
$3.00[0.13,70.30]$

$3.09[0.13,73.19]$

$1.21[0.67,2.19]$
$1.92[0.18,20.52]$

Total events:

Heterogeneity: $\mathrm{Tau}^{2}=0.00 ; \mathrm{Chi}^{2}=0.67, \mathrm{df}=2(\mathrm{P}=0.71) ; \mathrm{I}^{2}=0 \%$

Test for overall effect: $\mathrm{Z}=0.25(\mathrm{P}=0.80)$ 


\section{Analysis 1.13. (Continued)}

Heterogeneity: $\mathrm{Tau}^{2}=0.00 ; \mathrm{Chi}^{2}=0.67, \mathrm{df}=2(\mathrm{P}=0.71) ; \mathrm{I}^{2}=0 \%$

Test for overall effect: $\mathrm{Z}=0.25(\mathrm{P}=0.80)$

1.13.7 Protocol violation (e.g. refused treatment, withdrew consent)

$\begin{array}{lllll}\text { Almeida } 2006 & 26 & 55 & 29 & 56\end{array}$

Creytens 1980

25

3

$90.3 \%$

$0.91[0.63,1.33]$

Palomaki 1999

51

3

$25 \quad 4.4 \%$

$0.67[0.12,3.65]$

$0.32[0.03,2.98]$

Robinson 2000a

Robinson 2000b

15

$\begin{array}{lll}0 & 8 & 1.3 \%\end{array}$

$1.50[0.07,33.26]$

$1.69[0.08,37.26]$

Subtotal (95\% CI)

163

$1.3 \%$

0.89 [0.62, 1.27]

Total events:

31 35

Heterogeneity: $\mathrm{Tau}^{2}=0.00 ; \mathrm{Chi}^{2}=1.23, \mathrm{df}=4(\mathrm{P}=0.87) ; \mathrm{I}^{2}=0 \%$

Test for overall effect: $\mathrm{Z}=0.64(\mathrm{P}=0.52)$

$146 \mathbf{1 0 0 . 0 \%}$

35

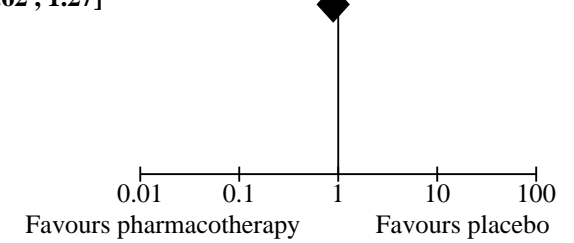

Analysis 1.14. Comparison 1: Pharmacological interventions (antidepressants) versus placebo, Outcome 14: Adverse events: leaving the study early (including death)

\begin{tabular}{|c|c|c|c|c|c|c|c|}
\hline \multirow[b]{2}{*}{ Study or Subgroup } & \multicolumn{2}{|c|}{ Pharmacotherapy } & \multicolumn{2}{|c|}{ Placebo } & \multicolumn{2}{|r|}{ Risk Ratio } & Risk Ratio \\
\hline & Events & Total & Events & Total & Weight & M-H, Random, $95 \%$ CI & M-H, Random, 95\% CI \\
\hline
\end{tabular}

\begin{tabular}{lrrrrr}
\hline 1.14.1 All dropouts and withdrawals & & & & \\
Almeida 2006 & 26 & 55 & 29 & 56 & $28.7 \%$ \\
Creytens 1980 & 4 & 25 & 4 & 25 & $2.5 \%$ \\
Dam 1996a & 2 & 18 & 0 & 8 & $0.5 \%$ \\
Dam 1996b & 3 & 17 & 1 & 9 & $0.9 \%$ \\
Palomaki 1999 & 9 & 51 & 10 & 49 & $6.2 \%$ \\
Rasmussen 2003 & 35 & 70 & 35 & 67 & $37.9 \%$ \\
Robinson 2000a & 4 & 17 & 1 & 8 & $1.0 \%$ \\
Robinson 2000b & 2 & 15 & 0 & 8 & $0.5 \%$ \\
Robinson 2008 & 7 & 58 & 5 & 59 & $3.4 \%$ \\
Roh 1996 & 2 & 32 & 3 & 33 & $1.4 \%$ \\
Tsai 2011 & 21 & 46 & 15 & 46 & $15.0 \%$ \\
Xu 2006 & 4 & 32 & 3 & 32 & $2.0 \%$ \\
Subtotal (95\% CI) & & $\mathbf{4 3 6}$ & & $\mathbf{4 0 0}$ & $\mathbf{1 0 0 . 0 \%}$ \\
Total events: & 119 & & 106 & &
\end{tabular}

Heterogeneity: $\mathrm{Tau}^{2}=0.00 ; \mathrm{Chi}^{2}=4.13, \mathrm{df}=11(\mathrm{P}=0.97) ; \mathrm{I}^{2}=0 \%$

Test for overall effect: $\mathrm{Z}=0.30(\mathrm{P}=0.76)$

Total $(95 \%$ CI $)$

436

$400 \quad 100.0 \%$

Total events:

119 106

Heterogeneity: $\mathrm{Tau}^{2}=0.00 ; \mathrm{Chi}^{2}=4.13, \mathrm{df}=11(\mathrm{P}=0.97) ; \mathrm{I}^{2}=0 \%$

Test for overall effect: $\mathrm{Z}=0.30(\mathrm{P}=0.76)$

Test for subgroup differences: Not applicable

$0.91[0.63,1.33]$

$1.00[0.28,3.56]$

$2.37[0.13,44.40]$

$1.59[0.19,13.15]$

$0.86[0.38,1.95]$

$0.96[0.69,1.33]$

$1.88[0.25,14.24]$

$2.81[0.15,52.38]$

$1.42[0.48,4.23]$

$0.69[0.12,3.85]$

$1.40[0.83,2.36]$

$1.33[0.32,5.49]$

$1.03[0.84,1.26]$

-
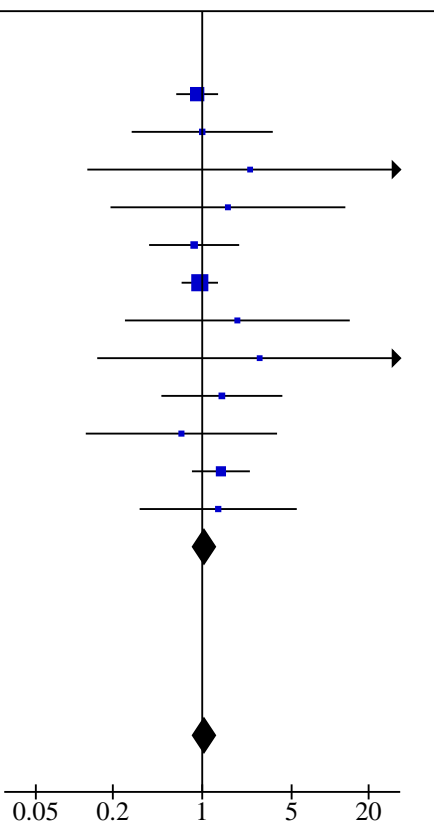

Favours pharmacotherapy 
Comparison 2. Psychological therapy versus standard care and/or attention control

\begin{tabular}{|c|c|c|c|c|}
\hline Outcome or subgroup title & $\begin{array}{l}\text { No. of } \\
\text { studies }\end{array}$ & $\begin{array}{l}\text { No. of } \\
\text { partici- } \\
\text { pants }\end{array}$ & Statistical method & Effect size \\
\hline $\begin{array}{l}\text { 2.1 Depression: meeting study criteria for } \\
\text { depression at end of treatment }\end{array}$ & 2 & 607 & Risk Ratio (M-H, Random, 95\% Cl) & $0.68[0.49,0.94]$ \\
\hline $\begin{array}{l}\text { 2.1.1 PSE (high score = probable psychi- } \\
\text { atric disorder) }\end{array}$ & 1 & 450 & Risk Ratio (M-H, Random, 95\% Cl) & $0.71[0.46,1.12]$ \\
\hline $\begin{array}{l}\text { 2.1.2 GHQ-28 (high score = greater psycho- } \\
\text { logical distress) }\end{array}$ & 1 & 157 & Risk Ratio (M-H, Random, 95\% Cl) & $0.64[0.39,1.03]$ \\
\hline $\begin{array}{l}2.2 \text { Scoring above cut-off points for a de- } \\
\text { pressive disorder at end of treatment }\end{array}$ & 0 & & Risk Ratio (M-H, Random, 95\% Cl) & $\begin{array}{l}\text { Totals not select- } \\
\text { ed }\end{array}$ \\
\hline $\begin{array}{l}2.3 \text { Depression: mean scores at end of } \\
\text { treatment }\end{array}$ & 2 & & Mean Difference (IV, Random, 95\% Cl) & $\begin{array}{l}\text { Totals not select- } \\
\text { ed }\end{array}$ \\
\hline 2.3.1 HADS (high score = more depressed) & 2 & & Mean Difference (IV, Random, 95\% Cl) & $\begin{array}{l}\text { Totals not select- } \\
\text { ed }\end{array}$ \\
\hline 2.3.2 MADRS (high score = more depressed) & 1 & & Mean Difference (IV, Random, 95\% Cl) & $\begin{array}{l}\text { Totals not select- } \\
\text { ed }\end{array}$ \\
\hline $\begin{array}{l}\text { 2.4 Depression: mean scores at end of fol- } \\
\text { low-up }\end{array}$ & 3 & & Mean Difference (IV, Random, 95\% Cl) & $\begin{array}{l}\text { Totals not select- } \\
\text { ed }\end{array}$ \\
\hline 2.4.1 HADS (high score = more depressed) & 3 & & Mean Difference (IV, Random, 95\% Cl) & $\begin{array}{l}\text { Totals not select- } \\
\text { ed }\end{array}$ \\
\hline 2.4.2 PHQ-9 (high score = more depressed) & 1 & & Mean Difference (IV, Random, 95\% Cl) & $\begin{array}{l}\text { Totals not select- } \\
\text { ed }\end{array}$ \\
\hline 2.4.3 MADRS (high score = more depressed) & 1 & & Mean Difference (IV, Random, 95\% Cl) & $\begin{array}{l}\text { Totals not select- } \\
\text { ed }\end{array}$ \\
\hline $\begin{array}{l}2.5 \text { Psychological distress: average change } \\
\text { in scores between baseline and end of } \\
\text { treatment }\end{array}$ & 2 & 607 & Mean Difference (IV, Random, 95\% Cl) & $-1.37[-2.27,-0.48]$ \\
\hline $\begin{array}{l}\text { 2.5.1 GHQ-28 (high score = greater psycho- } \\
\text { logical distress) }\end{array}$ & 2 & 607 & Mean Difference (IV, Random, 95\% Cl) & $-1.37[-2.27,-0.48]$ \\
\hline $\begin{array}{l}\text { 2.6 Psychological distress: mean scores at } \\
\text { end of treatment }\end{array}$ & 1 & & Mean Difference (IV, Fixed, 95\% CI) & $\begin{array}{l}\text { Totals not select- } \\
\text { ed }\end{array}$ \\
\hline $\begin{array}{l}\text { 2.6.1 GHQ-28 (high score = greater psycho- } \\
\text { logical distress) }\end{array}$ & 1 & & Mean Difference (IV, Fixed, 95\% CI) & $\begin{array}{l}\text { Totals not select- } \\
\text { ed }\end{array}$ \\
\hline $\begin{array}{l}2.7 \text { General Health: average change in } \\
\text { scores between baseline and end of treat- } \\
\text { ment }\end{array}$ & 1 & & Mean Difference (IV, Fixed, 95\% CI) & $\begin{array}{l}\text { Totals not select- } \\
\text { ed }\end{array}$ \\
\hline $\begin{array}{l}\text { 2.7.1 Nottingham Health Profile (high score } \\
=\text { better health) }\end{array}$ & 1 & & Mean Difference (IV, Fixed, 95\% CI) & $\begin{array}{l}\text { Totals not select- } \\
\text { ed }\end{array}$ \\
\hline
\end{tabular}




\begin{tabular}{|c|c|c|c|c|}
\hline Outcome or subgroup title & $\begin{array}{l}\text { No. of } \\
\text { studies }\end{array}$ & $\begin{array}{l}\text { No. of } \\
\text { partici- } \\
\text { pants }\end{array}$ & Statistical method & Effect size \\
\hline $\begin{array}{l}2.8 \text { General Health: Mean scores at end of } \\
\text { treatment }\end{array}$ & 1 & & Mean Difference (IV, Fixed, 95\% CI) & $\begin{array}{l}\text { Totals not select- } \\
\text { ed }\end{array}$ \\
\hline $\begin{array}{l}\text { 2.8.1 Nottingham Health Profile (high score } \\
=\text { better health) }\end{array}$ & 1 & & Mean Difference (IV, Fixed, 95\% CI) & $\begin{array}{l}\text { Totals not select- } \\
\text { ed }\end{array}$ \\
\hline $\begin{array}{l}\text { 2.9 Social activities: average change in } \\
\text { scores between baseline and end of treat- } \\
\text { ment }\end{array}$ & 1 & & Mean Difference (IV, Fixed, 95\% CI) & $\begin{array}{l}\text { Totals not select- } \\
\text { ed }\end{array}$ \\
\hline $\begin{array}{l}\text { 2.9.1 Frenchay Activities Index (high score = } \\
\text { better level of activity) }\end{array}$ & 1 & & Mean Difference (IV, Fixed, 95\% CI) & $\begin{array}{l}\text { Totals not select- } \\
\text { ed }\end{array}$ \\
\hline $\begin{array}{l}\text { 2.10 Social activities: mean scores at end of } \\
\text { treatment }\end{array}$ & 2 & 690 & Mean Difference (IV, Random, 95\% CI) & $-0.39[-3.81,3.03]$ \\
\hline $\begin{array}{l}\text { 2.10.1 Frenchay Activities Index (high score } \\
=\text { better level of activity) }\end{array}$ & 2 & 690 & Mean Difference (IV, Random, 95\% CI) & $-0.39[-3.81,3.03]$ \\
\hline $\begin{array}{l}\text { 2.11 Activities of daily living: Average } \\
\text { change in scores between baseline and } \\
\text { end of treatment }\end{array}$ & 3 & 847 & Mean Difference (IV, Random, 95\% CI) & $0.29[-0.18,0.77]$ \\
\hline $\begin{array}{l}\text { 2.11.1 Barthel Index (high score = more de- } \\
\text { pendent) }\end{array}$ & 3 & 847 & Mean Difference (IV, Random, 95\% CI) & $0.29[-0.18,0.77]$ \\
\hline $\begin{array}{l}\text { 2.12 Activities of daily living: mean scores } \\
\text { at end of treatment }\end{array}$ & 4 & & $\begin{array}{l}\text { Std. Mean Difference (IV, Random, } \\
95 \% \mathrm{CI})\end{array}$ & $\begin{array}{l}\text { Totals not select- } \\
\text { ed }\end{array}$ \\
\hline $\begin{array}{l}\text { 2.12.1 Barthel Index (high score = more de- } \\
\text { pendent) }\end{array}$ & 3 & & $\begin{array}{l}\text { Std. Mean Difference (IV, Random, } \\
95 \% \mathrm{CI})\end{array}$ & $\begin{array}{l}\text { Totals not select- } \\
\text { ed }\end{array}$ \\
\hline $\begin{array}{l}\text { 2.12.2 Modified Barthel Index (high score }= \\
\text { more dependent) }\end{array}$ & 1 & & $\begin{array}{l}\text { Std. Mean Difference (IV, Random, } \\
95 \% \mathrm{CI})\end{array}$ & $\begin{array}{l}\text { Totals not select- } \\
\text { ed }\end{array}$ \\
\hline $\begin{array}{l}\text { 2.12.3 Nottingham Extended Activities of } \\
\text { daily living (high score = more indepen- } \\
\text { dent) }\end{array}$ & 1 & & $\begin{array}{l}\text { Std. Mean Difference (IV, Random, } \\
95 \% \mathrm{CI})\end{array}$ & $\begin{array}{l}\text { Totals not select- } \\
\text { ed }\end{array}$ \\
\hline $\begin{array}{l}\text { 2.13 Activities of daily living: mean scores } \\
\text { at end of follow-up }\end{array}$ & 1 & & $\begin{array}{l}\text { Std. Mean Difference (IV, Fixed, 95\% } \\
\mathrm{Cl} \text { ) }\end{array}$ & $\begin{array}{l}\text { Totals not select- } \\
\text { ed }\end{array}$ \\
\hline $\begin{array}{l}\text { 2.13.1 Modified Barthel Index (high score }= \\
\text { more dependent) }\end{array}$ & 1 & & $\begin{array}{l}\text { Std. Mean Difference (IV, Fixed, 95\% } \\
\mathrm{Cl} \text { ) }\end{array}$ & $\begin{array}{l}\text { Totals not select- } \\
\text { ed }\end{array}$ \\
\hline $\begin{array}{l}\text { 2.13.2 Nottingham Extended Activities of } \\
\text { daily living (high score = more indepen- } \\
\text { dent) }\end{array}$ & 1 & & $\begin{array}{l}\text { Std. Mean Difference (IV, Fixed, 95\% } \\
\mathrm{Cl})\end{array}$ & $\begin{array}{l}\text { Totals not select- } \\
\text { ed }\end{array}$ \\
\hline $\begin{array}{l}\text { 2.14 Anxiety: mean scores at end of treat- } \\
\text { ment }\end{array}$ & 2 & & $\begin{array}{l}\text { Std. Mean Difference (IV, Random, } \\
95 \% \mathrm{CI})\end{array}$ & $\begin{array}{l}\text { Totals not select- } \\
\text { ed }\end{array}$ \\
\hline 2.14.1 HADS (high score = more anxious) & 2 & & $\begin{array}{l}\text { Std. Mean Difference (IV, Random, } \\
95 \% \mathrm{CI})\end{array}$ & $\begin{array}{l}\text { Totals not select- } \\
\text { ed }\end{array}$ \\
\hline
\end{tabular}




\begin{tabular}{|c|c|c|c|c|}
\hline Outcome or subgroup title & $\begin{array}{l}\text { No. of } \\
\text { studies }\end{array}$ & $\begin{array}{l}\text { No. of } \\
\text { partici- } \\
\text { pants }\end{array}$ & Statistical method & Effect size \\
\hline 2.14.2 STI Trait Anxiety & 1 & & $\begin{array}{l}\text { Std. Mean Difference (IV, Random, } \\
95 \% \mathrm{CI})\end{array}$ & $\begin{array}{l}\text { Totals not select- } \\
\text { ed }\end{array}$ \\
\hline 2.14.3 STI State Anxiety & 1 & & $\begin{array}{l}\text { Std. Mean Difference (IV, Random, } \\
95 \% \mathrm{CI})\end{array}$ & $\begin{array}{l}\text { Totals not select- } \\
\text { ed }\end{array}$ \\
\hline $\begin{array}{l}2.15 \text { Anxiety: mean scores at end of fol- } \\
\text { low-up }\end{array}$ & 3 & & $\begin{array}{l}\text { Std. Mean Difference (IV, Random, } \\
95 \% \mathrm{CI})\end{array}$ & $\begin{array}{l}\text { Totals not select- } \\
\text { ed }\end{array}$ \\
\hline 2.15.1 HADS (high score = more anxious) & 3 & & $\begin{array}{l}\text { Std. Mean Difference (IV, Random, } \\
95 \% \mathrm{CI})\end{array}$ & $\begin{array}{l}\text { Totals not select- } \\
\text { ed }\end{array}$ \\
\hline 2.15.2 STI Trait Anxiety & 1 & & $\begin{array}{l}\text { Std. Mean Difference (IV, Random, } \\
95 \% \mathrm{CI})\end{array}$ & $\begin{array}{l}\text { Totals not select- } \\
\text { ed }\end{array}$ \\
\hline 2.15.3 STI State Anxiety & 1 & & $\begin{array}{l}\text { Std. Mean Difference (IV, Random, } \\
95 \% \mathrm{Cl} \text { ) }\end{array}$ & $\begin{array}{l}\text { Totals not select- } \\
\text { ed }\end{array}$ \\
\hline 2.16 Adverse events: death & 5 & 975 & Risk Ratio (M-H, Random, 95\% Cl) & $1.18[0.73,1.91]$ \\
\hline 2.16.1 At end of treatment & 5 & 975 & Risk Ratio (M-H, Random, 95\% Cl) & $1.18[0.73,1.91]$ \\
\hline 2.17 Adverse events: all & 2 & & Risk Ratio (M-H, Random, 95\% Cl) & Subtotals only \\
\hline 2.17.1 Recurrent stroke & 2 & 212 & Risk Ratio (M-H, Random, 95\% Cl) & $2.61[0.88,7.70]$ \\
\hline $\begin{array}{l}\text { 2.17.2 Vascular events - not stroke (e.g. } \\
\text { transient ischaemic attack) }\end{array}$ & 1 & 157 & Risk Ratio (M-H, Random, 95\% Cl) & $6.23[0.77,50.59]$ \\
\hline $\begin{array}{l}\text { 2.18 Adverse events: leaving the study ear- } \\
\text { ly (including death) }\end{array}$ & 6 & 1030 & Risk Ratio (M-H, Random, 95\% Cl) & $1.30[0.90,1.88]$ \\
\hline 2.18.1 All dropouts and withdrawals & 6 & 1030 & Risk Ratio (M-H, Random, 95\% Cl) & $1.30[0.90,1.88]$ \\
\hline
\end{tabular}


Analysis 2.1. Comparison 2: Psychological therapy versus standard care and/or attention control, Outcome 1: Depression: meeting study criteria for depression at end of treatment

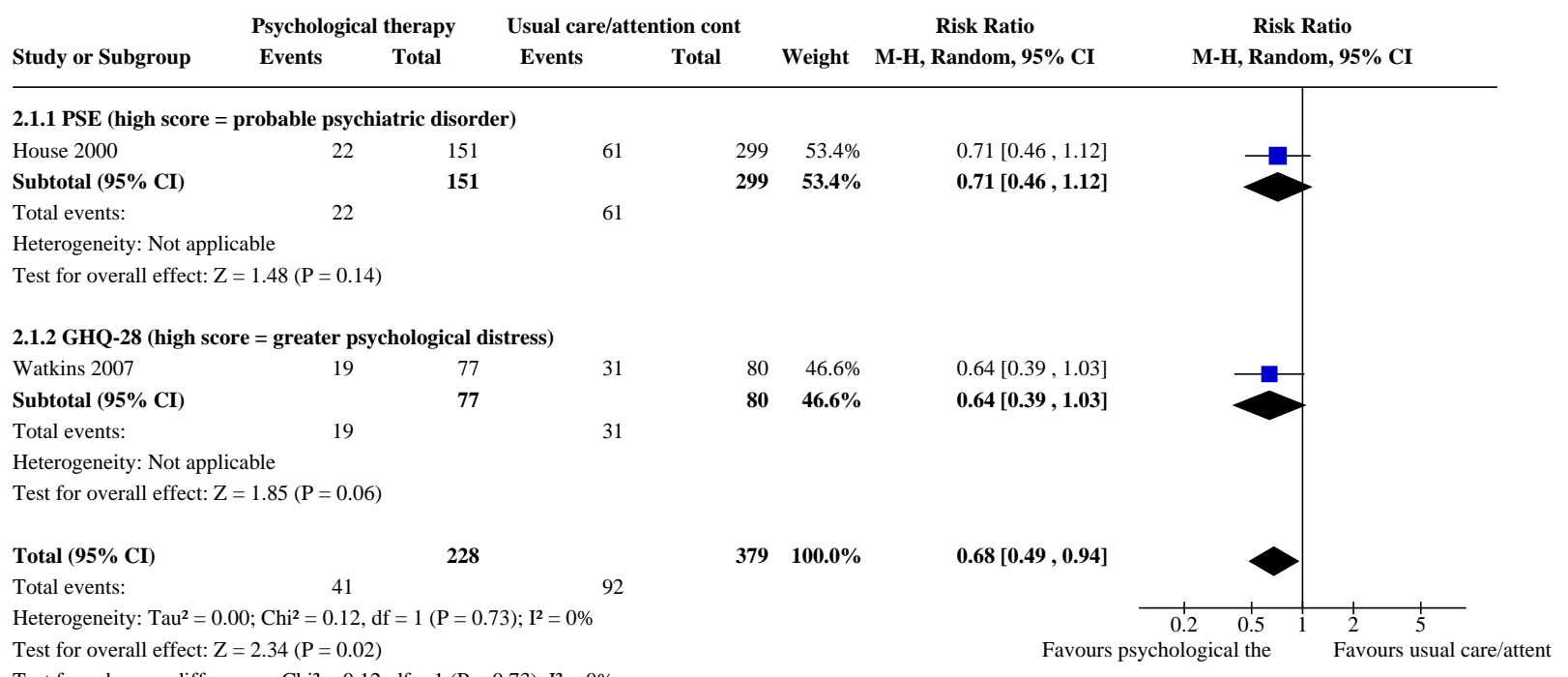

Analysis 2.2. Comparison 2: Psychological therapy versus standard care and/or attention control, Outcome 2: Scoring above cut-off points for a depressive disorder at end of treatment

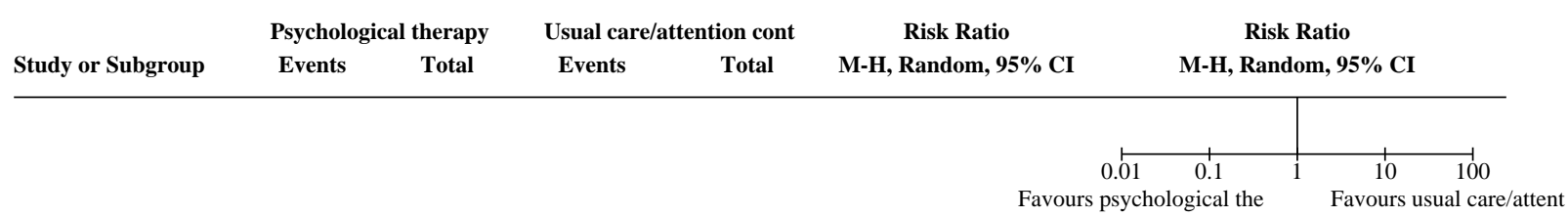

Analysis 2.3. Comparison 2: Psychological therapy versus standard care and/ or attention control, Outcome 3: Depression: mean scores at end of treatment

\begin{tabular}{|c|c|c|c|c|c|c|c|c|c|}
\hline \multirow[b]{2}{*}{ Study or Subgroup } & \multicolumn{3}{|c|}{ Psychological therapy } & \multicolumn{3}{|c|}{ Usual care/attention cont } & \multirow{2}{*}{$\begin{array}{c}\text { Mean Difference } \\
\text { IV, Random, 95\% CI }\end{array}$} & \multirow{2}{*}{\multicolumn{2}{|c|}{$\begin{array}{c}\text { Mean Difference } \\
\text { IV, Random, 95\% CI }\end{array}$}} \\
\hline & Mean & SD & Total & Mean & SD & Total & & & \\
\hline \multicolumn{10}{|c|}{ 2.3.1 HADS (high score $=$ more depressed) } \\
\hline Hoffman 2015 & 7.5 & 0.45 & 11 & 5.85 & 0.6 & 5 & $1.65[1.06,2.24]$ & & + \\
\hline Wichowicz 2017 & 2.93 & 2.89 & 51 & 4.56 & 4.08 & 49 & $-1.63[-3.02,-0.24]$ & 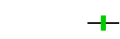 & \\
\hline \multicolumn{10}{|c|}{ 2.3.2 MADRS (high score $=$ more depressed $)$} \\
\hline Hoffman 2015 & 13.1 & 0.69 & 11 & 13.53 & 0.93 & 5 & $-0.43[-1.34,0.48]$ & 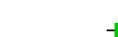 & \\
\hline
\end{tabular}


Analysis 2.4. Comparison 2: Psychological therapy versus standard care and/ or attention control, Outcome 4: Depression: mean scores at end of follow-up

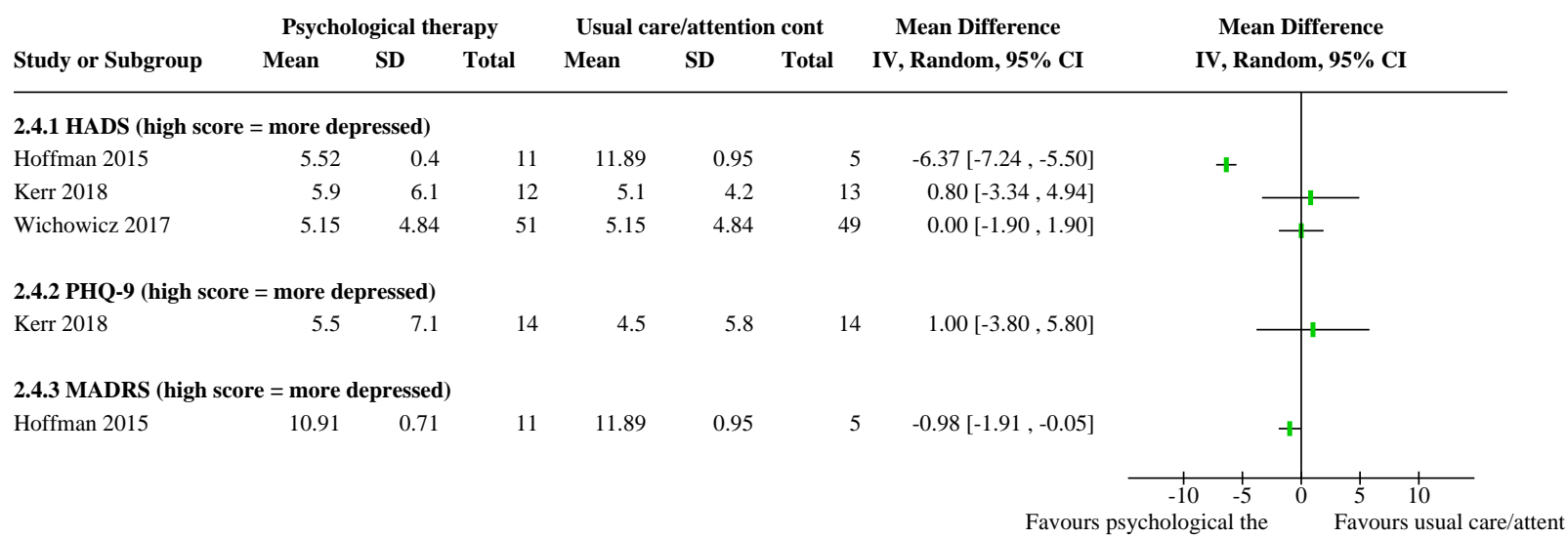

Analysis 2.5. Comparison 2: Psychological therapy versus standard care and/or attention control, Outcome 5: Psychological distress: average change in scores between baseline and end of treatment

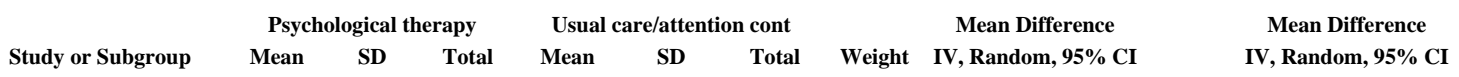

2.5.1 GHQ-28 (high score = greater psychological distress)

$\begin{array}{lrrrrrrrr}\text { 2.5.1 GHQ-28 (high score } & \text { greater psychological distress) } & & & & & \\ \text { House 2000 } & -2.13 & 5.57 & 151 & -0.72 & 5.53 & 299 & 67.6 \% & -1.41[-2.50,-0.32] \\ \text { Watkins 2007 } & 1.5 & 4.5 & 77 & 2.8 & 5.5 & 80 & 32.4 \% & -1.30[-2.87,0.27] \\ \text { Subtotal (95\% CI) } & & & \mathbf{2 2 8} & & & \mathbf{3 7 9} & \mathbf{1 0 0 . 0 \%} & \mathbf{- 1 . 3 7 [ - 2 . 2 7 , - 0 . 4 8 ]}\end{array}$

Subtotal (95\% CI)

228

$379100.0 \%$

$-1.37[-2.27,-0.48]$

Heterogeneity: $\mathrm{Tau}^{2}=0.00 ; \mathrm{Chi}^{2}=0.01, \mathrm{df}=1(\mathrm{P}=0.91) ; \mathrm{I}^{2}=0 \%$

Test for overall effect: $\mathrm{Z}=3.01(\mathrm{P}=0.003)$

Total (95\% CI) $\quad 228$

Heterogeneity: $\mathrm{Tau}^{2}=0.00 ; \mathrm{Chi}^{2}=0.01, \mathrm{df}=1(\mathrm{P}=0.91) ; \mathrm{I}^{2}=0 \%$

Test for overall effect: $\mathrm{Z}=3.01(\mathrm{P}=0.003)$

Test for subgroup differences: Not applicable
$379 \quad 100.0 \% \quad-1.37[-2.27,-0.48]$

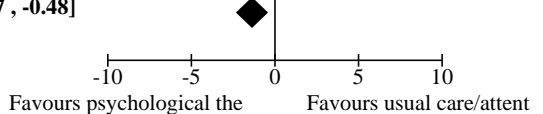

Analysis 2.6. Comparison 2: Psychological therapy versus standard care and/or attention control, Outcome 6: Psychological distress: mean scores at end of treatment

\begin{tabular}{|c|c|c|c|c|c|c|c|c|c|c|}
\hline \multirow[b]{2}{*}{ Study or Subgroup } & \multicolumn{3}{|c|}{ Psychological therapy } & \multicolumn{3}{|c|}{ Usual care/attention cont } & \multirow{2}{*}{$\begin{array}{l}\text { Mean Difference } \\
\text { IV, Fixed, 95\% CI }\end{array}$} & \multirow{2}{*}{\multicolumn{2}{|c|}{$\begin{array}{c}\text { Mean Difference } \\
\text { IV, Fixed, 95\% CI }\end{array}$}} & \\
\hline & Mean & SD & Total & Mean & SD & Total & & & & \\
\hline \multicolumn{10}{|c|}{ 2.6.1 GHQ-28 (high score = greater psychological distress) } & \\
\hline House 2000 & 4.5 & 5.26 & 151 & 5.64 & 5.34 & 299 & $-1.14[-2.17,-0.11]$ & $\rightarrow$ & & \\
\hline
\end{tabular}


Analysis 2.7. Comparison 2: Psychological therapy versus standard care and/or attention control, Outcome 7: General Health: average change in scores between baseline and end of treatment

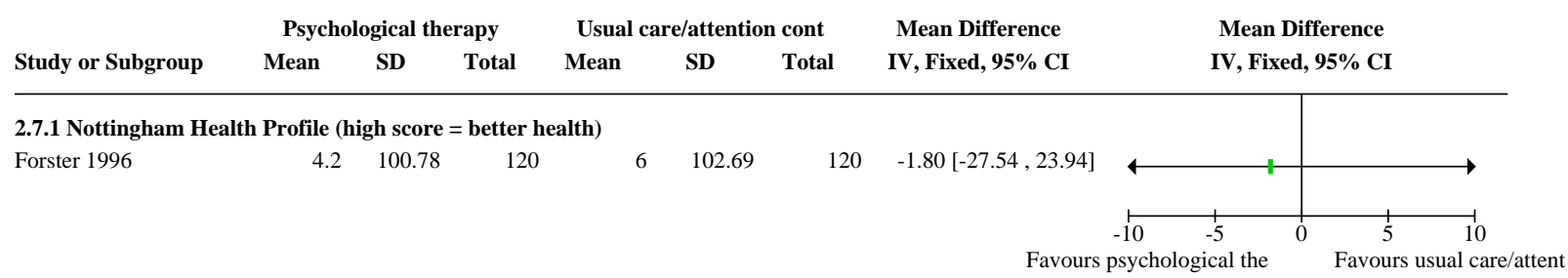

Analysis 2.8. Comparison 2: Psychological therapy versus standard care and/ or attention control, Outcome 8: General Health: Mean scores at end of treatment

\begin{tabular}{|c|c|c|c|c|c|c|c|c|}
\hline \multirow[b]{2}{*}{ Study or Subgroup } & \multicolumn{3}{|c|}{ Psychological therapy } & \multicolumn{3}{|c|}{ Usual care/attention cont } & \multirow{2}{*}{$\begin{array}{c}\text { Mean Difference } \\
\text { IV, Fixed, 95\% CI }\end{array}$} & \multirow{2}{*}{$\begin{array}{c}\text { Mean Difference } \\
\text { IV, Fixed, 95\% CI }\end{array}$} \\
\hline & Mean & SD & Total & Mean & SD & Total & & \\
\hline \multicolumn{9}{|c|}{ 2.8.1 Nottingham Health Profile (high score = better health) } \\
\hline Forster 1996 & 115.9 & 105.8 & 120 & 111.3 & 98.4 & 120 & $4.60[-21.25,30.45]$ & 1 \\
\hline
\end{tabular}

Analysis 2.9. Comparison 2: Psychological therapy versus standard care and/or attention control, Outcome 9: Social activities: average change in scores between baseline and end of treatment

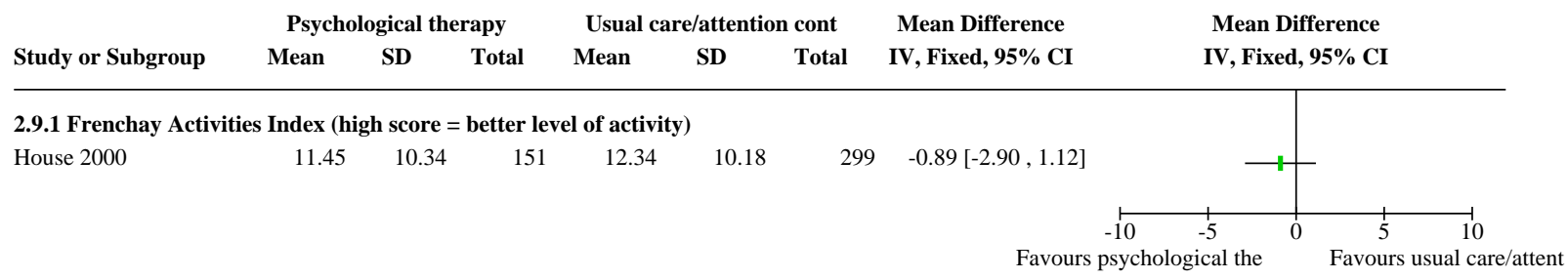

Analysis 2.10. Comparison 2: Psychological therapy versus standard care and/ or attention control, Outcome 10: Social activities: mean scores at end of treatment

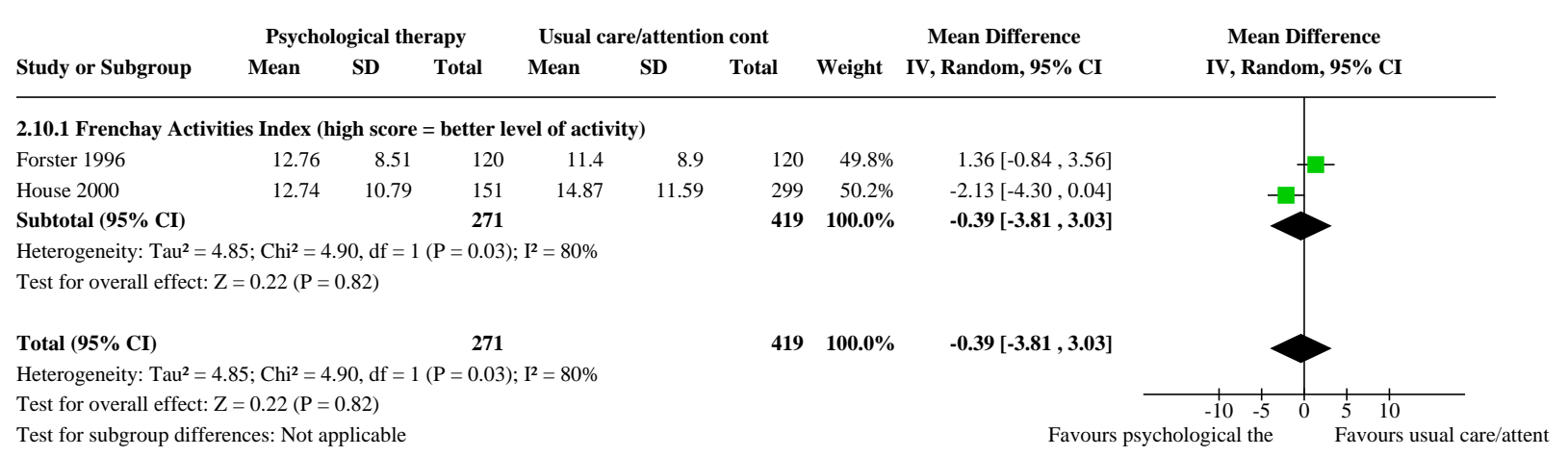


Analysis 2.11. Comparison 2: Psychological therapy versus standard care and/or attention control, Outcome 11: Activities of daily living: Average change in scores between baseline and end of treatment

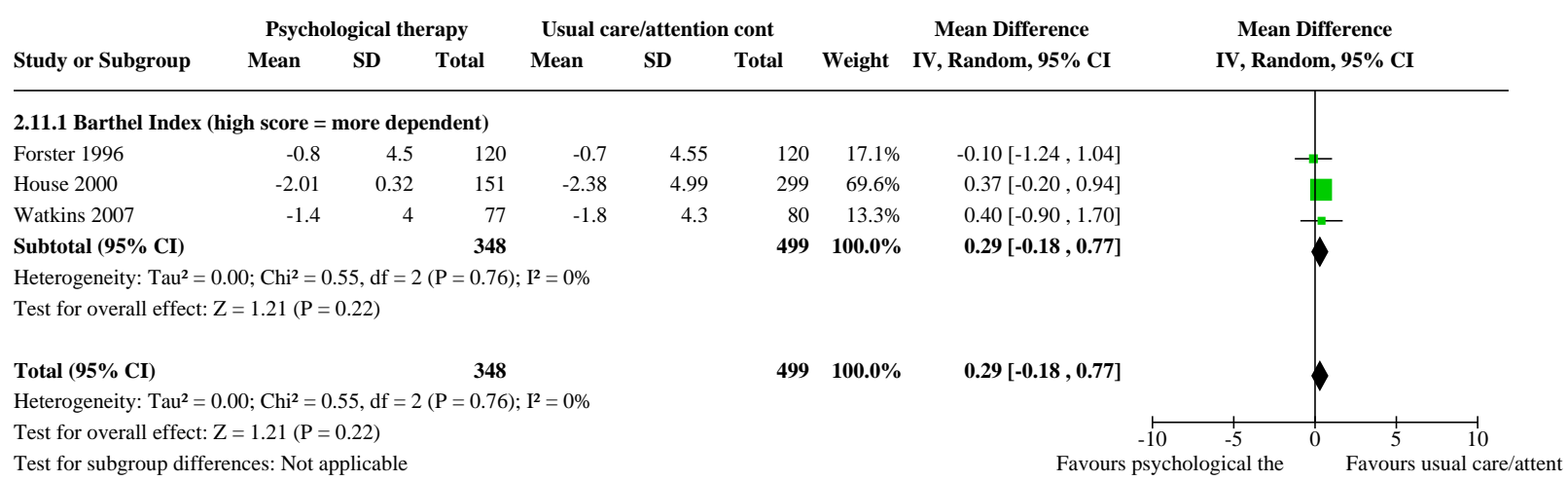

Analysis 2.12. Comparison 2: Psychological therapy versus standard care and/or attention control, Outcome 12: Activities of daily living: mean scores at end of treatment

\begin{tabular}{lcccccccc} 
& \multicolumn{2}{c}{ Psychological therapy } & \multicolumn{2}{c}{ Usual care/attention cont } & \multicolumn{2}{c}{ Std. Mean Difference } & Std. Mean Difference \\
Study or Subgroup & Mean & SD & Total & Mean & SD & Total & IV, Random, 95\% CI & IV, Random, 95\% CI
\end{tabular}

2.12.1 Barthel Index (high score $=$ more dependent)

$\begin{array}{llllllll}\text { Forster } 1996 & 16.1 & 4.5 & 120 & 15.6 & 4.7 & 120 & 0.11[-0.14,0.36]\end{array}$

$\begin{array}{llllllll}\text { House } 2000 & 15.4 & 4.78 & 151 & 16.14 & 4.58 & 299 & -0.16[-0.35,0.04]\end{array}$

$\begin{array}{llllllll}\text { Watkins } 2007 & 17.9 & 3.2 & 77 & 17.1 & 4.1 & 80 & 0.22[-0.10,0.53]\end{array}$

2.12.2 Modified Barthel Index (high score $=$ more dependent)

$\begin{array}{llllllll}\text { Hoffman } 2015 & 75.89 & 3.63 & 11 & 68.77 & 4.49 & 5 & 1.73[0.47,2.99]\end{array}$

2.12.3 Nottingham Extended Activities of daily living (high score = more independent)

$\begin{array}{llllllll}\text { Hoffman } 2015 & 40.39 & 3.76 & 11 & 43.45 & 5 & 5 & -0.70[-1.79,0.40]\end{array}$

Analysis 2.13. Comparison 2: Psychological therapy versus standard care and/or attention control, Outcome 13: Activities of daily living: mean scores at end of follow-up

\begin{tabular}{ccccccccc} 
& \multicolumn{2}{c}{ Psychological therapy } & \multicolumn{2}{c}{ Usual care/attention cont } & Std. Mean Difference & Std. Mean Difference \\
Study or Subgroup & Mean & SD & Total & Mean & SD & Total & IV, Fixed, 95\% CI & IV, Fixed, 95\% CI
\end{tabular}

2.13.1 Modified Barthel Index (high score = more dependent)

$\begin{array}{llllllll}\text { Hoffman } 2015 & 82.38 & 3.54 & 9 & 82.94 & 4.37 & 5 & -0.14[-1.23,0.96]\end{array}$

2.13.2 Nottingham Extended Activities of daily living (high score = more independent)

$\begin{array}{llllllll}\text { Hoffman } 2015 & 49.41 & 3.46 & 9 & 51.51 & 4.61 & 5 & -0.51[-1.62,0.61]\end{array}$

Favours psychological the Favours usual care/attent 
Analysis 2.14. Comparison 2: Psychological therapy versus standard care and/ or attention control, Outcome 14: Anxiety: mean scores at end of treatment

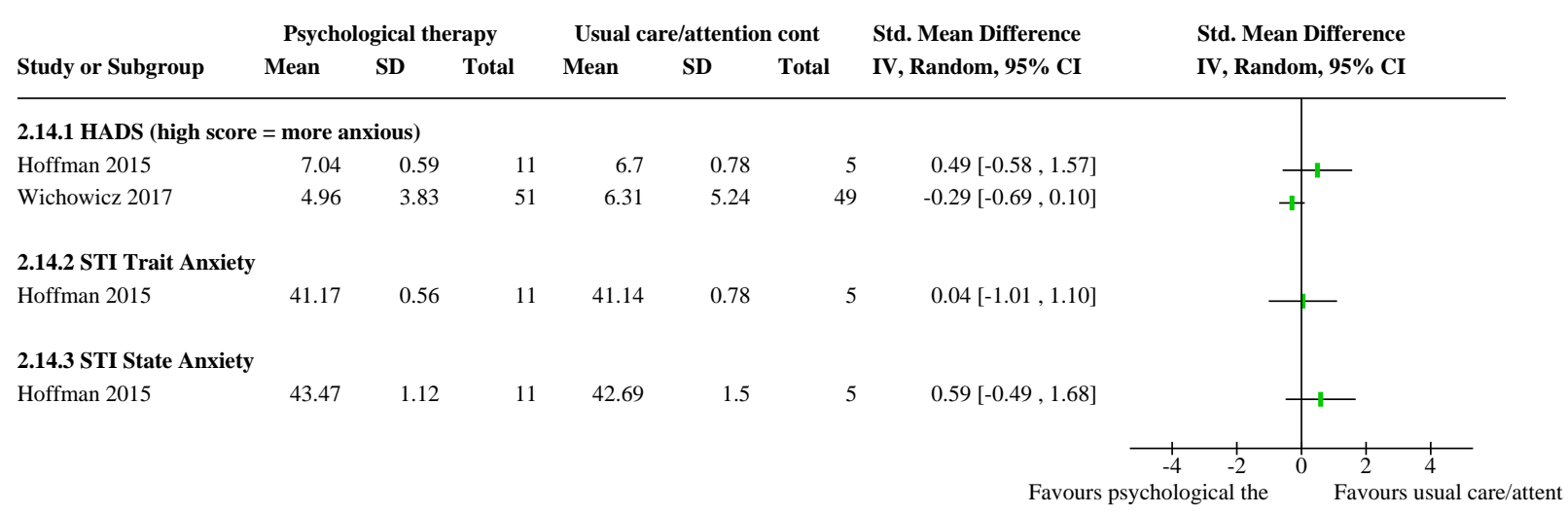

Analysis 2.15. Comparison 2: Psychological therapy versus standard care and/ or attention control, Outcome 15: Anxiety: mean scores at end of follow-up

\begin{tabular}{|c|c|c|c|c|c|c|c|c|}
\hline \multirow[b]{2}{*}{ Study or Subgroup } & \multicolumn{3}{|c|}{ Psychological therapy } & \multicolumn{3}{|c|}{ Usual care/attention cont } & \multirow{2}{*}{$\begin{array}{l}\text { Std. Mean Difference } \\
\text { IV, Random, 95\% CI }\end{array}$} & \multirow{2}{*}{$\begin{array}{l}\text { Std. Mean Difference } \\
\text { IV, Random, 95\% CI }\end{array}$} \\
\hline & Mean & SD & Total & Mean & SD & Total & & \\
\hline \multicolumn{9}{|c|}{ 2.15.1 HADS (high score = more anxious) } \\
\hline Hoffman 2015 & 5.32 & 0.46 & 11 & 6 & 0.6 & 5 & $-1.28[-2.45,-0.10]$ & -1 \\
\hline Kerr 2018 & 7.9 & 6.5 & 14 & 6.8 & 6.4 & 14 & $0.17[-0.58,0.91]$ & - \\
\hline Wichowicz 2017 & 6.93 & 5.87 & 51 & 6.93 & 5.87 & 49 & $0.00[-0.39,0.39]$ & 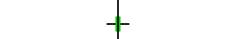 \\
\hline \multicolumn{9}{|c|}{ 2.15.2 STI Trait Anxiety } \\
\hline Hoffman 2015 & 41.05 & 0.53 & 11 & 40.88 & 0.73 & 5 & $0.27[-0.79,1.33]$ & 1 \\
\hline \multicolumn{9}{|c|}{ 2.15.3 STI State Anxiety } \\
\hline Hoffman 2015 & 42.11 & 1.08 & 11 & 41.06 & 1.4 & 5 & $0.84[-0.27,1.95]$ & -1 \\
\hline
\end{tabular}

\section{Analysis 2.16. Comparison 2: Psychological therapy versus standard care and/or attention control, Outcome 16: Adverse events: death}

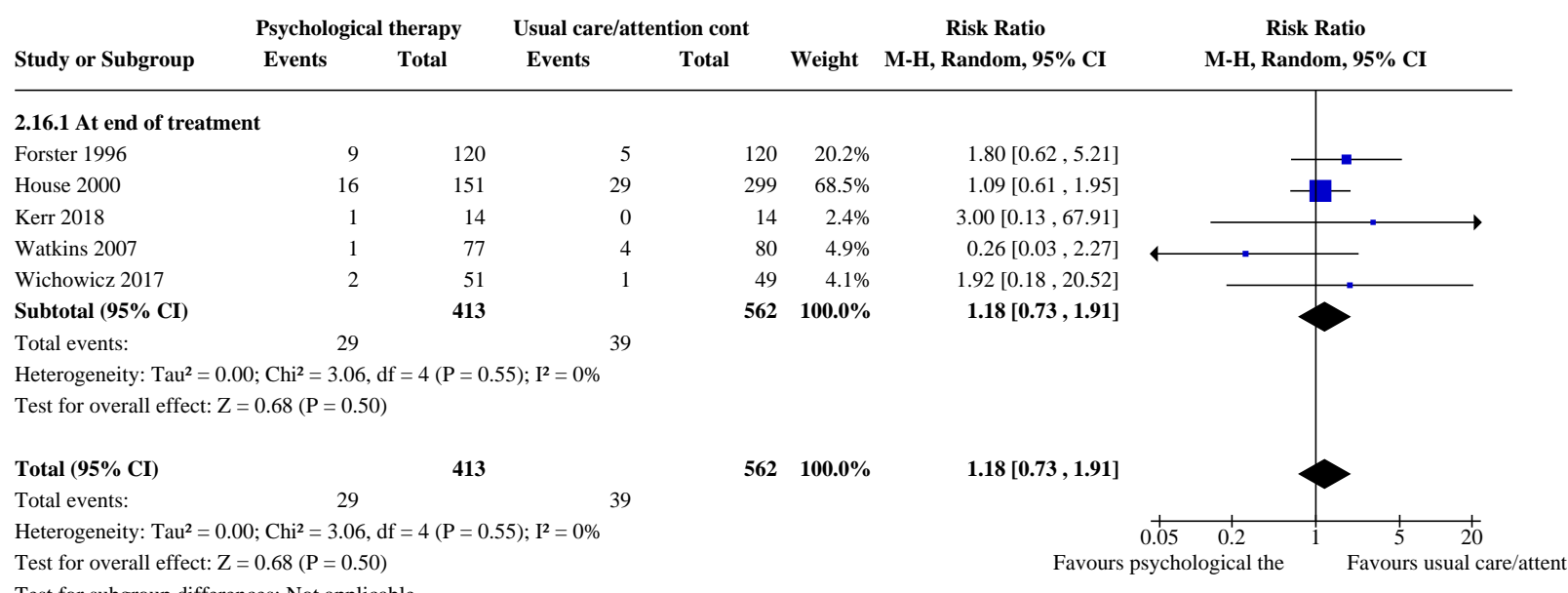




\section{Analysis 2.17. Comparison 2: Psychological therapy versus standard} care and/or attention control, Outcome 17: Adverse events: all

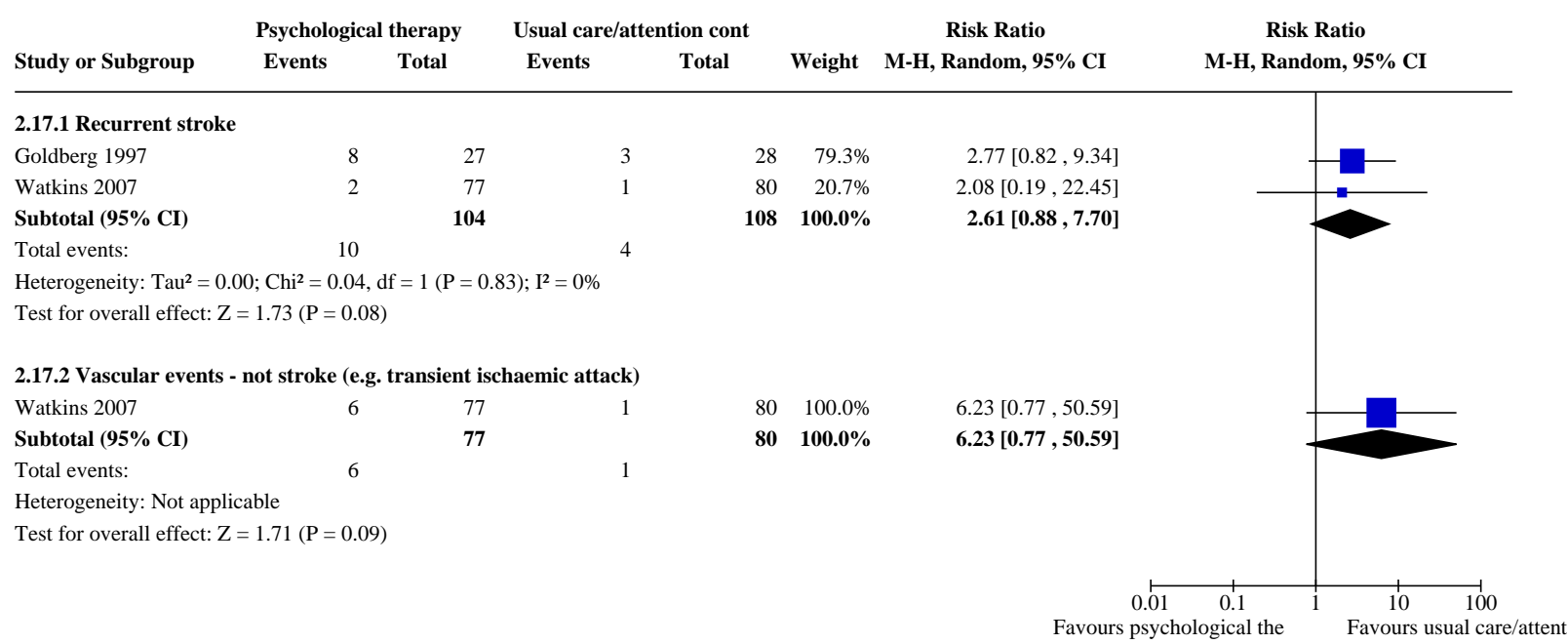

Analysis 2.18. Comparison 2: Psychological therapy versus standard care and/or attention control, Outcome 18: Adverse events: leaving the study early (including death)

\begin{tabular}{|c|c|c|c|c|c|c|c|c|}
\hline \multirow[b]{2}{*}{ Study or Subgroup } & \multicolumn{2}{|c|}{ Psychological therapy } & \multicolumn{2}{|c|}{ Usual care/attention cont } & \multirow[b]{2}{*}{ Weight } & \multirow{2}{*}{$\begin{array}{c}\text { Risk Ratio } \\
\text { M-H, Random, 95\% CI }\end{array}$} & \multirow{2}{*}{\multicolumn{2}{|c|}{$\begin{array}{c}\text { Risk Ratio } \\
\text { M-H, Random, 95\% CI }\end{array}$}} \\
\hline & Events & Total & Events & Total & & & & \\
\hline \multicolumn{9}{|c|}{ 2.18.1 All dropouts and withdrawals } \\
\hline Forster 1996 & 10 & 120 & 5 & 120 & $10.5 \%$ & $2.00[0.70,5.68]$ & - & \\
\hline Goldberg 1997 & 6 & 27 & 8 & 28 & $13.0 \%$ & $0.78[0.31,1.95]$ & $\longrightarrow$ & \\
\hline House 2000 & 34 & 151 & 49 & 299 & $37.8 \%$ & $1.37[0.93,2.03]$ & & \\
\hline Kerr 2018 & 8 & 14 & 2 & 14 & $6.6 \%$ & $4.00[1.03,15.60]$ & 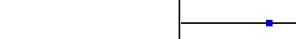 & $\longrightarrow$ \\
\hline Watkins 2007 & 1 & 77 & 4 & 80 & $2.8 \%$ & $0.26[0.03,2.27]$ & $\longleftarrow$ & \\
\hline Wichowicz 2017 & 21 & 51 & 17 & 49 & $29.4 \%$ & $1.19[0.72,1.97]$ & - & \\
\hline Subtotal (95\% CI) & & 440 & & 590 & $100.0 \%$ & $1.30[0.90,1.88]$ & & \\
\hline Total events: & 80 & & 85 & & & & & \\
\hline \multicolumn{9}{|c|}{ Heterogeneity: $\mathrm{Tau}^{2}=0.05 ; \mathrm{Chi}^{2}=6.80, \mathrm{df}=5(\mathrm{P}=0.24) ; \mathrm{I}^{2}=27 \%$} \\
\hline \multicolumn{9}{|c|}{ Test for overall effect: $\mathrm{Z}=1.41(\mathrm{P}=0.16)$} \\
\hline Total $(95 \%$ CI $)$ & & 440 & & 590 & $100.0 \%$ & $1.30[0.90,1.88]$ & & \\
\hline Total events: & 80 & & 85 & & & & & \\
\hline \multicolumn{7}{|c|}{ Heterogeneity: $\mathrm{Tau}^{2}=0.05 ; \mathrm{Chi}^{2}=6.80, \mathrm{df}=5(\mathrm{P}=0.24) ; \mathrm{I}^{2}=27 \%$} & $\begin{array}{llll}1 & 1 & 1 & 1 \\
0.1 & 0.2 & 0.5 & 1\end{array}$ & $5 \frac{1}{10}$ \\
\hline \multicolumn{4}{|c|}{ Test for overall effect: $\mathrm{Z}=1.41(\mathrm{P}=0.16)$} & & & Favours & sychological the & usual c \\
\hline
\end{tabular}

\section{Comparison 3. Pharmacological interventions (psychostimulants) versus placebo}

\begin{tabular}{lllll}
\hline Outcome or subgroup title & $\begin{array}{l}\text { No. of } \\
\text { studies }\end{array}$ & $\begin{array}{l}\text { No. of } \\
\text { partici- } \\
\text { pants }\end{array}$ & Statistical method & Effect size \\
\hline $\begin{array}{l}\text { 3.1 Depression: average change in scores be- } \\
\text { tween baseline and end of treatment }\end{array}$ & 1 & Mean Difference (IV, Fixed, 95\% Cl) & $\begin{array}{l}\text { Totals not select- } \\
\text { ed }\end{array}$ \\
\hline $\begin{array}{llll}\text { 3.1.1 HDRS (high score = more depressed) } \\
\text { 3.1.2 ZDS (high score = more depressed) }\end{array}$ & 1 & Mean Difference (IV, Fixed, 95\% Cl) & $\begin{array}{l}\text { Totals not select- } \\
\text { ed }\end{array}$ \\
\hline
\end{tabular}

Pharmacological, psychological and non-invasive brain stimulation interventions for preventing depression after stroke (Review) 


\begin{tabular}{|c|c|c|c|c|}
\hline Outcome or subgroup title & $\begin{array}{l}\text { No. of } \\
\text { studies }\end{array}$ & $\begin{array}{l}\text { No. of } \\
\text { partici- } \\
\text { pants }\end{array}$ & Statistical method & Effect size \\
\hline $\begin{array}{l}\text { 3.2 Cognition: average change in scores be- } \\
\text { tween baseline and end of treatment }\end{array}$ & 1 & & Mean Difference (IV, Fixed, 95\% CI) & $\begin{array}{l}\text { Totals not select- } \\
\text { ed }\end{array}$ \\
\hline $\begin{array}{l}\text { 3.2.1 MMSE (low score = cognitive impair- } \\
\text { ment) }\end{array}$ & 1 & & Mean Difference (IV, Fixed, 95\% CI) & $\begin{array}{l}\text { Totals not select- } \\
\text { ed }\end{array}$ \\
\hline $\begin{array}{l}\text { 3.3 Disability: average change in scores be- } \\
\text { tween baseline and end of treatment }\end{array}$ & 1 & & Mean Difference (IV, Fixed, 95\% Cl) & $\begin{array}{l}\text { Totals not select- } \\
\text { ed }\end{array}$ \\
\hline $\begin{array}{l}\text { 3.3.1 Fugl-Meyer Scale (high score = better } \\
\text { function) }\end{array}$ & 1 & & Mean Difference (IV, Fixed, 95\% CI) & $\begin{array}{l}\text { Totals not select- } \\
\text { ed }\end{array}$ \\
\hline $\begin{array}{l}\text { 3.3.2 Functional Independence Measure } \\
\text { (high score = independence) }\end{array}$ & 1 & & Mean Difference (IV, Fixed, 95\% CI) & $\begin{array}{l}\text { Totals not select- } \\
\text { ed }\end{array}$ \\
\hline 3.4 Adverse events: death & 1 & & Risk Ratio (M-H, Fixed, 95\% Cl) & Subtotals only \\
\hline 3.4.1 At end of treatment & 1 & 21 & Risk Ratio (M-H, Fixed, 95\% Cl) & Not estimable \\
\hline $\begin{array}{l}\text { 3.5 Adverse events: leaving the study early } \\
\text { (including death) }\end{array}$ & 1 & & Risk Ratio (M-H, Fixed, 95\% Cl) & $\begin{array}{l}\text { Totals not select- } \\
\text { ed }\end{array}$ \\
\hline 3.5.1 All dropouts and withdrawals & 1 & & Risk Ratio (M-H, Fixed, 95\% Cl) & $\begin{array}{l}\text { Totals not select- } \\
\text { ed }\end{array}$ \\
\hline
\end{tabular}

\section{Analysis 3.1. Comparison 3: Pharmacological interventions (psychostimulants) versus placebo, Outcome 1: Depression: average change in scores between baseline and end of treatment}

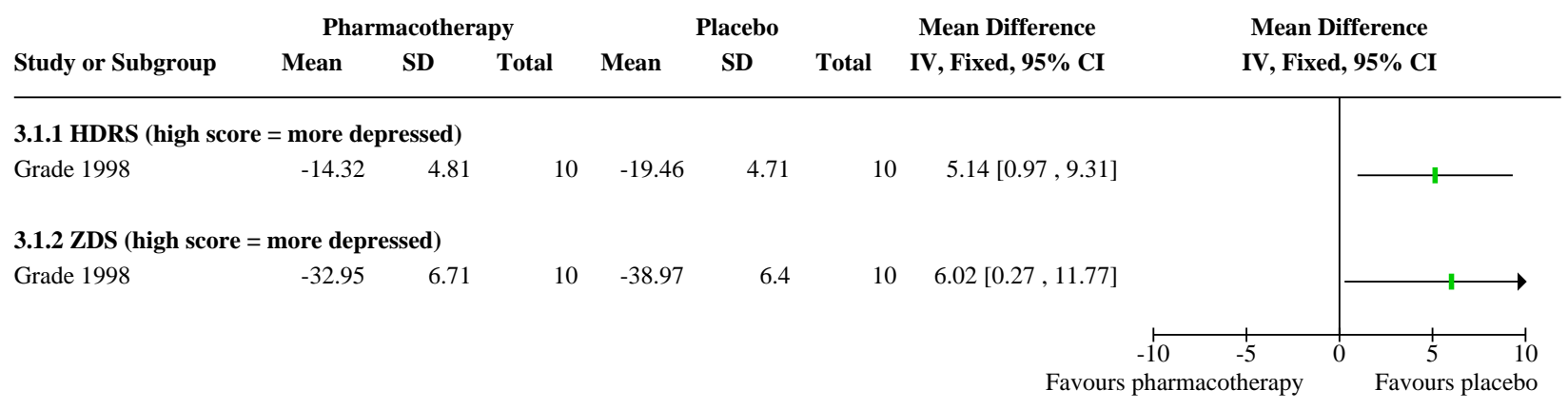


Analysis 3.2. Comparison 3: Pharmacological interventions (psychostimulants) versus placebo, Outcome 2: Cognition: average change in scores between baseline and end of treatment

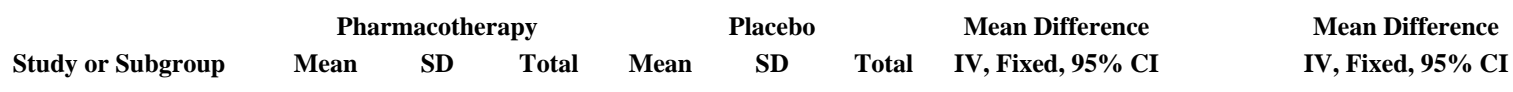

3.2.1 MMSE (low score = cognitive impairment)

Grade 1998

$23.55 \quad 2.19$

$10 \quad 24.15 \quad 2.09$

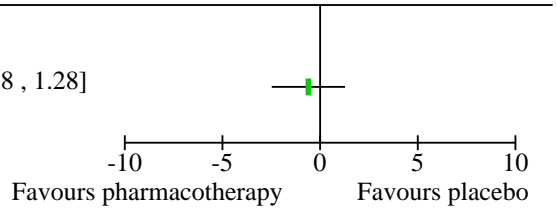

Analysis 3.3. Comparison 3: Pharmacological interventions (psychostimulants) versus placebo, Outcome 3: Disability: average change in scores between baseline and end of treatment

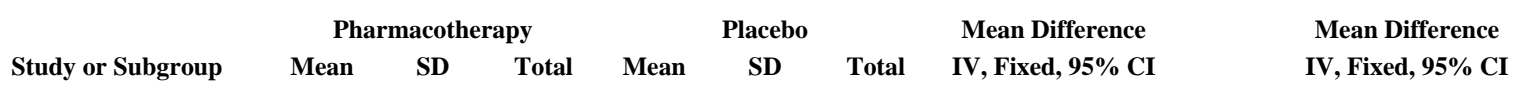

3.3.1 Fugl-Meyer Scale (high score $=$ better function)

$\begin{array}{llllllll}\text { Grade } 1998 & 55.26 & 22.37 & 10 & 37.78 & 15.76 & 10 & 17.48[0.52,34.44]\end{array}$

3.3.2 Functional Independence Measure (high score $=$ independence)

$\begin{array}{llllllll}\text { Grade } 1998 & 116.46 & 11.29 & 10 & 104.95 & 10.75 & 10 & 11.51[1.85,21.17]\end{array}$

$11.51[1.85,21.17]$

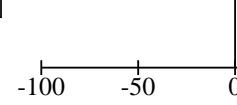

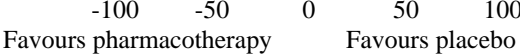

Analysis 3.4. Comparison 3: Pharmacological interventions (psychostimulants) versus placebo, Outcome 4: Adverse events: death

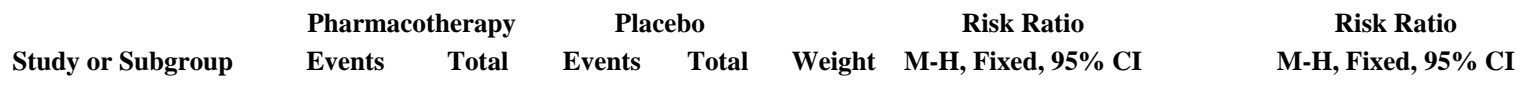

3.4.1 At end of treatment

Grade 1998

Subtotal (95\% CI)

0

10

10
10

0

11

11

Not estimable

Total events:

0

0

Not estimable

Heterogeneity: Not applicable

Test for overall effect: Not applicable

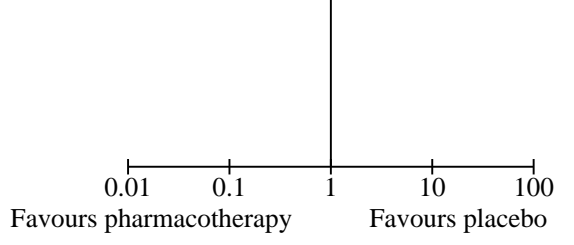


Analysis 3.5. Comparison 3: Pharmacological interventions (psychostimulants) versus placebo, Outcome 5: Adverse events: leaving the study early (including death)

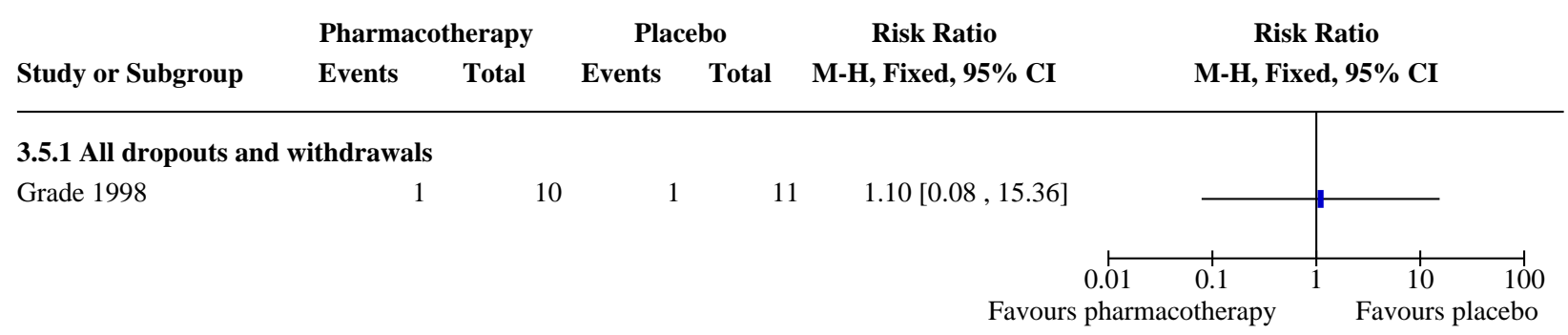

ADDITIONAL TABLES

Table 1. Characteristics of dropout studies

\begin{tabular}{|c|c|c|c|c|c|}
\hline Study ID & Methods & Participants & Interventions & $\begin{array}{l}\text { Out- } \\
\text { comes }\end{array}$ & Notes \\
\hline $\begin{array}{l}\text { Braman- } \\
\text { ti } 1989\end{array}$ & $\begin{array}{l}\text { Study de- } \\
\text { sign: par- } \\
\text { allel de- } \\
\text { sign } \\
\text { Number } \\
\text { of arms: } 2 \\
\text { Experi- } \\
\text { mental } \\
\text { arm: pro- } \\
\text { tirelin tar- } \\
\text { trate } \\
\text { (TRH-T) } \\
\text { Control } \\
\text { arm: } \\
\text { placebo }\end{array}$ & $\begin{array}{l}\text { Geographical location: Italy } \\
\text { Setting: unclear } \\
\text { Number of participants: } 30 \\
\text { Stroke criteria: acute stroke } \\
\text { Method of stroke diagnosis: not reported } \\
\text { Inclusion criteria: not reported } \\
\text { Exclusion criteria: not reported } \\
\text { Depression criteria: not reported } \\
\text { Number included in treatment group: unclear } \\
\text { ( } 63 \% \text { male, mean age } 72.2, \text { SD not reported of } \\
\text { the overall cohort) } \\
\text { Number included in control group: unclear } \\
\text { (63\% men, mean age } 72.2, \text { SD not reported of } \\
\text { the overall cohort) }\end{array}$ & $\begin{array}{l}\text { Treatment: protirelin tartrate } \\
\text { (TRHT) } 2 \mathrm{mg} / \text { day } \\
\text { Control: placebo } \\
\text { Duration: } 2 \text { weeks } \\
\text { Follow-up: none }\end{array}$ & $\begin{array}{l}\text { De- } \\
\text { pres- } \\
\text { sion } \\
\text { mea- } \\
\text { sured } \\
\text { using } \\
\text { the } \\
\text { HDRS }\end{array}$ & $\begin{array}{l}\text { Results } \\
\text { not } \\
\text { available } \\
\text { in for- } \\
\text { mat suit- } \\
\text { able for } \\
\text { the re- } \\
\text { view }\end{array}$ \\
\hline $\begin{array}{l}\text { Downes } \\
1995\end{array}$ & $\begin{array}{l}\text { Study de- } \\
\text { sign: par- } \\
\text { allel de- } \\
\text { sign } \\
\text { Number } \\
\text { of arms: } 3 \\
\text { Experi- } \\
\text { mental } \\
\text { arm 1: in- } \\
\text { formation } \\
\text { + coun- } \\
\text { selling } \\
\text { Experi- } \\
\text { mental } \\
\text { arm 2: in- } \\
\text { formation } \\
\text { pack } \\
\text { Control } \\
\text { arm: usual } \\
\text { care }\end{array}$ & $\begin{array}{l}\text { Geographical location: UK } \\
\text { Setting: outpatient } \\
\text { Number of participants: } 62 \\
\text { Stroke criteria: not reported } \\
\text { Method of stroke diagnosis: not reported } \\
\text { Inclusion criteria: } 1 \text { ) lived at home; } 2 \text { ) had an } \\
\text { informal carer; 3) stroke increase mRS; } 4 \text { ) post- } \\
\text { stroke mRS score of } 2 \text { to } 5 \\
\text { Exclusion criteria: } 1 \text { ) not living at home; } 2 \text { ) not } \\
\text { having an informal carer; } 3 \text { ) having no increase } \\
\text { in disability or change in lifestyle/dependency } \\
\text { Depression criteria: HADS score > } 11 \\
\text { Number included in treatment group 1: } 22 \\
\text { (50\% men, age not reported) } \\
\text { Number included in treatment group 2: } 22 \\
\text { (55\% men, age not reported) } \\
\text { Number included in control group: } 18 \text { ( } 44 \% \\
\text { men, age not reported) }\end{array}$ & $\begin{array}{l}\text { Treatment 1: information } \\
\text { plus counselling. Egan's prob- } \\
\text { lem-solving approach, individ- } \\
\text { ual is helped to explore con- } \\
\text { cerns, clarify problems, set } \\
\text { goals, and take appropriate ac- } \\
\text { tion. Protocol discussed first } \\
\text { and formulated into a counsel- } \\
\text { lor/client contract. Information } \\
\text { pack containing information on } \\
\text { physical, cognitive, behavioural } \\
\text { and emotional effects of stroke, } \\
\text { carer well-being, and local ser- } \\
\text { vices. } \\
\text { Treatment 2: information only: } \\
\text { information pack containing in- } \\
\text { formation on physical, cogni- } \\
\text { tive,behavioural, and emotional } \\
\text { effects of stroke, carer well-be- } \\
\text { ing, and local services. } \\
\text { Control: usual care, no visit(s) or } \\
\text { information pack provided }\end{array}$ & $\begin{array}{l}\text { De- } \\
\text { pres- } \\
\text { sion } \\
\text { mea- } \\
\text { sured } \\
\text { using } \\
\text { the } \\
\text { HADS- } \\
\text { De- } \\
\text { pres- } \\
\text { sion } \\
\text { Anxi- } \\
\text { ety } \\
\text { mea- } \\
\text { sured } \\
\text { using } \\
\text { the } \\
\text { HADS- } \\
\text { Anxi- } \\
\text { ety }\end{array}$ & $\begin{array}{l}\text { Unable } \\
\text { to iso- } \\
\text { late } \\
\text { outcome } \\
\text { data for } \\
\text { non-de- } \\
\text { pressed } \\
\text { partic- } \\
\text { ipants } \\
\text { at ran- } \\
\text { domisa- } \\
\text { tion }\end{array}$ \\
\hline
\end{tabular}


Duration: information session consisted of 1 visit and provision of the information pack. Counselling consisted of up to 8 counselling sessions over 4 to 6 months Administered by: nurse counsellor

Supervision: unclear

Follow-up: none

\begin{tabular}{|c|c|c|c|c|c|}
\hline $\begin{array}{l}\text { Fried- } \\
\text { land } \\
1992\end{array}$ & $\begin{array}{l}\text { Study de- } \\
\text { sign: par- } \\
\text { allel de- } \\
\text { sign } \\
\text { Number } \\
\text { of arms: } 2 \\
\text { Experi- } \\
\text { mental } \\
\text { arm: psy- } \\
\text { choedu- } \\
\text { cational } \\
\text { support } \\
\text { Control } \\
\text { arm: } \\
\text { usual care }\end{array}$ & $\begin{array}{l}\text { Geographical location: Canada } \\
\text { Setting: outpatient } \\
\text { Number of participants: } 88 \\
\text { Stroke criteria: all subtypes } \\
\text { Method of stroke diagnosis: via clinical signs } \\
\text { Inclusion criteria: 1) completed formal inpa- } \\
\text { tient rehabilitation and a period of rehabilita- } \\
\text { tion provided by a home care programme } \\
\text { Exclusion criteria: } 1 \text { ) history of psychiatric ad- } \\
\text { mission, 2) previously on antidepressant med- } \\
\text { ication, 3) aphasia with limited ability to com- } \\
\text { municate verbally } \\
\text { Depression criteria: unclear } \\
\text { Number included in treatment group: } 48 \text { ( } 44 \% \\
\text { men, mean age } 69 \text { years, SD 11) } \\
\text { Number included in control group: } 40 \text { ( } 44 \% \\
\text { men, mean age } 69 \text { years, SD 11) }\end{array}$ & $\begin{array}{l}\text { Treatment: psychoeducational, } \\
\text { with participant and members } \\
\text { of their support team; work to } \\
\text { improve social support, estab- } \\
\text { lish new supports, emotional } \\
\text { support offered } \\
\text { Control: usual care, no visits } \\
\text { Duration: treatment continued } \\
\text { for } 6 \text { to } 12 \text { sessions over approx- } \\
\text { imately } 3 \text { months } \\
\text { Administered by: specially } \\
\text { trained social support interven- } \\
\text { tion therapist } \\
\text { Supervision: unclear } \\
\text { Follow-up: } 6 \text { months ( } 3 \text { months } \\
\text { post intervention) }\end{array}$ & $\begin{array}{l}\text { De- } \\
\text { pres- } \\
\text { sion } \\
\text { (dif- } \\
\text { fer- } \\
\text { ence } \\
\text { in } \\
\text { scores } \\
\text { at end } \\
\text { of } \\
\text { treat- } \\
\text { ment) } \\
\text { mea- } \\
\text { sured } \\
\text { using } \\
\text { the } \\
\text { GHQ-28 }\end{array}$ & $\begin{array}{l}\text { Results } \\
\text { not } \\
\text { available }\end{array}$ \\
\hline $\begin{array}{l}\text { Graffin- } \\
\text { go } 2003\end{array}$ & $\begin{array}{l}\begin{array}{l}\text { Study de- } \\
\text { sign: }\end{array} \\
\text { parallel } \\
\text { design } \\
\text { Number } \\
\text { of arms: } 2 \\
\text { Experi- } \\
\text { mental } \\
\text { arm: ser- } \\
\text { traline } \\
\text { (SSRI) } \\
\text { Control } \\
\text { arm: } \\
\text { matched } \\
\text { placebo }\end{array}$ & $\begin{array}{l}\text { Geographical location: unclear } \\
\text { Setting: unclear } \\
\text { Number of participants: unclear } \\
\text { Stroke criteria: unclear } \\
\text { Method of stroke diagnosis: unclear } \\
\text { Inclusion criteria: unclear } \\
\text { Exclusion criteria: unclear } \\
\text { Depression criteria: unclear } \\
\text { Number included in treatment group: unclear } \\
\text { Number included in control group: unclear }\end{array}$ & $\begin{array}{l}\text { Treatment: sertraline (SSRI) } \\
\text { Control: matched placebo } \\
\text { Duration: unclear } \\
\text { Follow-up: unclear }\end{array}$ & $\begin{array}{l}\text { De- } \\
\text { pres- } \\
\text { sion: } \\
\text { un- } \\
\text { clear } \\
\text { what } \\
\text { mea- } \\
\text { sure } \\
\text { was } \\
\text { used }\end{array}$ & $\begin{array}{l}\text { Results } \\
\text { not } \\
\text { available }\end{array}$ \\
\hline $\begin{array}{l}\text { Hadidi } \\
2014\end{array}$ & $\begin{array}{l}\text { Study de- } \\
\text { sign: par- } \\
\text { allel de- } \\
\text { sign }\end{array}$ & $\begin{array}{l}\text { Geographical location: USA } \\
\text { Setting: inpatient } \\
\text { Number of participants: } 22\end{array}$ & $\begin{array}{l}\text { Treatment: one-on-one prob- } \\
\text { lem solving therapy sessions } \\
\text { lasting } 1-2 \text { hours. Therapy en- } \\
\text { tails providing patient informa- } \\
\text { tion on impact and guidance to }\end{array}$ & $\begin{array}{l}\text { De- } \\
\text { pres- } \\
\text { sion } \\
\text { mea- } \\
\text { sured }\end{array}$ & $\begin{array}{l}\text { Unable } \\
\text { to iso- } \\
\text { late out- } \\
\text { come } \\
\text { data for }\end{array}$ \\
\hline
\end{tabular}


Table 1. Characteristics of dropout studies (Continued) of arms: 2 stroke $<48$ hours

Experi- Method of stroke diagnosis: not reported mental Inclusion criteria: 1) Mini-Cog score of $3 ; \geq 50$

arm: $\quad$ years of age; 2 ) able to read and write in English

prob-

lem-solv-

ing thera-

py (PST)

Con-

trol arm:

weekly

telephone

calls
Number Stroke criteria: first time diagnosis of ischaemic
Exclusion criteria: 1) previous history of mental

health problems; 2 ) diagnosis of severe apha-

sia as identified by a speech pathologist; 3 )

haemorrhagic stroke or transient ischaemic

attack; 4) medical instability requiring transfer

to critical care

Depression criteria: CES-D score measured at baseline but patients recruited regardless of their CES-D score. If CES-D score $>10$, or suicidal ideation the primary physician was notified

Number included in treatment group: 11 (18\% men, mean age 73 )

Number included in control group: 11 (45\% men, mean age 69) enable the patient to: identify and define the problem; brainstorm all potential solutions; select the most appropriate and feasible solution; create and implement a SMART (Specific, Measureable,

Achievable, Realistic and Timely) goal; evaluate and re-view progress in follow-up sessions Administered by: a doctoral nursing student who received PST training through a 13- module online program adapted from a standard 3-day in person training

Supervision: principal investigator who had undergone in person PST training

Intervention fidelity: not reported

Control: weekly telephone calls to assess CES-D and FIM scores Duration: once per week for 10 weeks

Follow-up: 3 months

Treatment: oral escitalopram

(SSRI) $10 \mathrm{mg} /$ day

Control: placebo

Duration: 3 months

Follow-up: 6 months

of arms: 2 bral haemorrhage

Experi- $\quad$ Method of stroke diagnosis: confirmed by MRI mental or CT

arm:

escitalo-

pram

(SSRI)

Con-

trol arm:

placebo
Inclusion criteria: 1 ) $>20$ years, 2) had an acute ischaemic stroke or intracerebral haemorrhage within the previous 21 days, 3) mRS score of 2 or greater at the time of screening, 4) agreed to participate

Exclusion criteria: 1) history of diagnosed depression or other psychiatric diseases before the index stroke, 2) severe dementia, 3) cognitive dysfunction (stages 5-7 of the Global Deterioration Scale), 4) aphasia, 5) on antimigraine or antiepileptic medication, 6) suicidal thoughts (a combined MADRS score $>8,7$ ) pregnant or lactating, 8) participation in another clinical trial

Depression criteria: MADRS

Number included in treatment group: 241

Number included in control group: 237 using non-de-

the pressed

CES-D partic-

- Im- ipants

pair- at ran-

ment domisa-

mea- tion

sured

using

the

FIM

- Leav-

ing

the

trial

early

\begin{tabular}{ll} 
De- & Unable \\
pres- & to iso- \\
sion & late \\
(fre- & outcome \\
quen- & data for \\
cy of & non- de- \\
mod- & pressed \\
erate & partic- \\
or se- & ipants \\
vere & at ran- \\
de- & domisa- \\
pres- & tion \\
sive & \\
symp- & \\
toms) & \\
mea- & \\
sured & \\
using & \\
the & \\
(MADRS) \\
Im- & \\
pair- & \\
ment & \\
mea- & \\
sured & \\
using & \\
the & \\
NIHSS & \\
Func- & \\
tional & \\
ca- & \\
\hline
\end{tabular}


Table 1. Characteristics of dropout studies (Continued)

\begin{tabular}{|c|c|c|c|c|c|}
\hline & & & & $\begin{array}{l}\text { pacity } \\
\text { mea- } \\
\text { sured } \\
\text { using } \\
\text { the } \\
\text { mRS } \\
\text { - Activ- } \\
\text { ities } \\
\text { of } \\
\text { daily } \\
\text { living } \\
\text { mea- } \\
\text { sured } \\
\text { using } \\
\text { the BI } \\
\text { - Ad- } \\
\text { verse } \\
\text { events }\end{array}$ & \\
\hline $\begin{array}{l}\text { Leathley } \\
2003\end{array}$ & $\begin{array}{l}\text { Study de- } \\
\text { sign: } \\
\text { parallel } \\
\text { design } \\
\text { Number } \\
\text { of arms: } 4 \\
\text { Experi- } \\
\text { mental } \\
\text { arm 1: so- } \\
\text { cial sup- } \\
\text { port } \\
\text { Experi- } \\
\text { mental } \\
\text { arm 2: } \\
\text { psycho- } \\
\text { logical } \\
\text { support } \\
\text { (cogni- } \\
\text { tive thera- } \\
\text { py based } \\
\text { problem } \\
\text { solving) } \\
\text { Experi- } \\
\text { mental } \\
\text { arm 3: } \\
\text { social } \\
\text { support } \\
\text { and psy- } \\
\text { chological } \\
\text { support } \\
\text { Control } \\
\text { arm: usual } \\
\text { care }\end{array}$ & $\begin{array}{l}\text { Geographical location: UK } \\
\text { Setting: outpatient } \\
\text { Number of participants: unclear } \\
\text { Stroke criteria: unclear } \\
\text { Method of stroke diagnosis: unclear } \\
\text { Inclusion criteria: unclear } \\
\text { Exclusion criteria: unclear } \\
\text { Number included in treatment group 1: unclear } \\
\text { Number included in treatment group 2: unclear } \\
\text { Number included in treatment group 3: unclear } \\
\text { Number included in control group: unclear }\end{array}$ & $\begin{array}{l}\text { Treatment 1: social support (in- } \\
\text { formation, practical advice, ser- } \\
\text { vice liaison) } \\
\text { Treatment 2: psychological sup- } \\
\text { port (cognitive therapy based } \\
\text { problem solving) } \\
\text { Treatment 3: social support and } \\
\text { psychological support } \\
\text { Control: usual care, no visits } \\
\text { Duration: unclear } \\
\text { Administered by: unclear } \\
\text { Supervision: unclear } \\
\text { Follow-up: unclear }\end{array}$ & $\begin{array}{l}\text { De- } \\
\text { pres- } \\
\text { sion } \\
\text { (dif- } \\
\text { fer- } \\
\text { ence } \\
\text { be- } \\
\text { tween } \\
\text { groups } \\
\text { at end } \\
\text { of } \\
\text { treat- } \\
\text { ment) } \\
\text { mea- } \\
\text { sured } \\
\text { using } \\
\text { the } \\
\text { GHQ-12 } \\
\text { total } \\
\text { score }\end{array}$ & $\begin{array}{l}\text { Results } \\
\text { not } \\
\text { available } \\
\text { in for- } \\
\text { mat suit- } \\
\text { able } \\
\text { for this } \\
\text { review }\end{array}$ \\
\hline
\end{tabular}


Table 1. Characteristics of dropout studies (Continued)

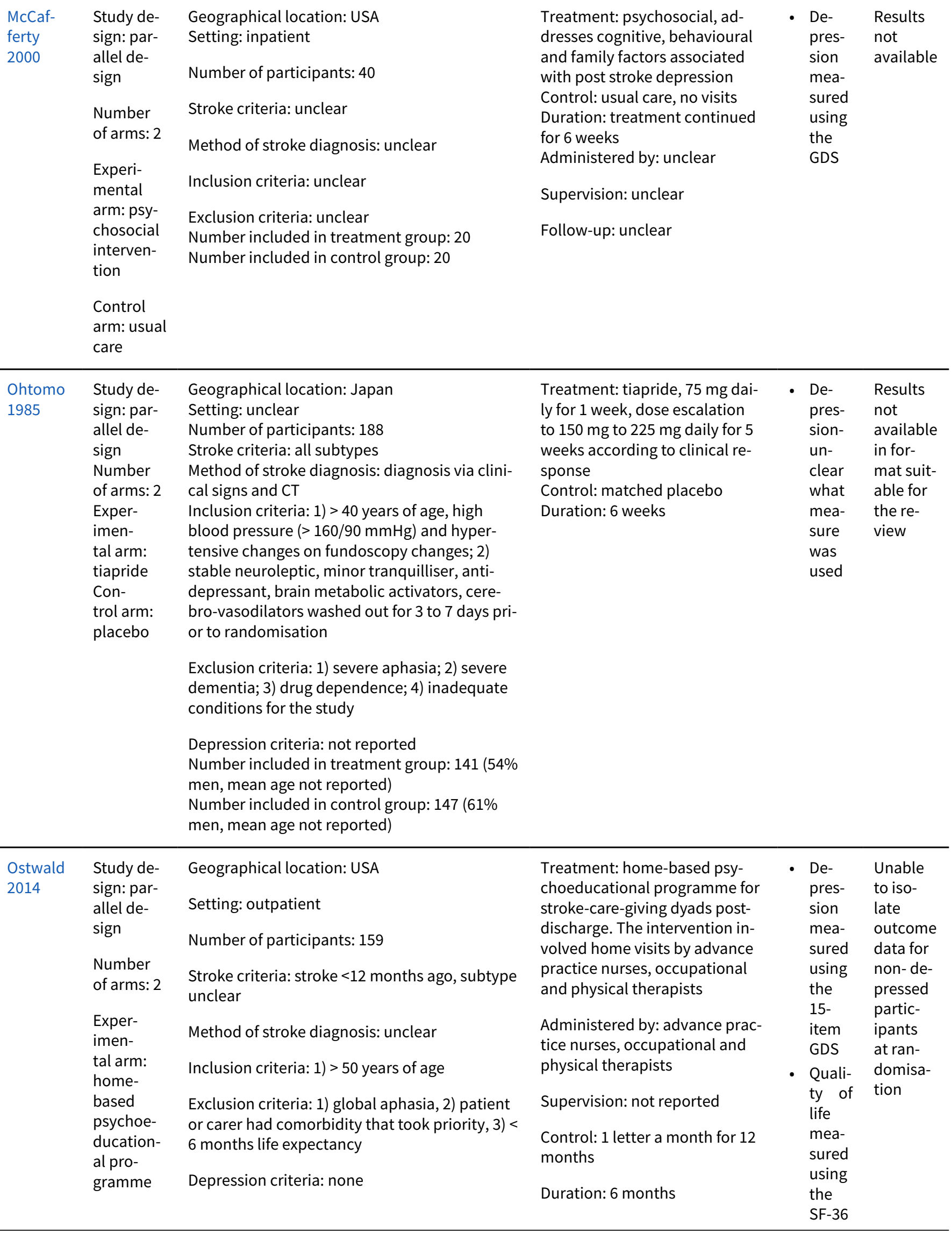


Table 1. Characteristics of dropout studies (Continued)

Con- Number included in treatment group: 79 (69\%

trol arm:

month-

ly mailed

letter

men, mean age 67 years)

Number included in control group: 80 (81\%

men, mean age 66 years)
Follow-up: 6 months

- Func-

tional

ca-

pacity

mea-

sured

using

the

FIM

physi-

cal

and

cog-

nitive

sub-

scales

\begin{tabular}{|c|c|c|c|c|c|}
\hline $\begin{array}{l}\text { Raffaele } \\
1996\end{array}$ & $\begin{array}{l}\text { Study de- } \\
\text { sign: par- } \\
\text { allel de- } \\
\text { sign } \\
\text { Number } \\
\text { of arms: } 2 \\
\text { Experi- } \\
\text { mental } \\
\text { arm: tra- } \\
\text { zodone } \\
\text { Control } \\
\text { arm: } \\
\text { placebo }\end{array}$ & $\begin{array}{l}\text { Geographical location: Italy } \\
\text { Setting: outpatient } \\
\text { Number of participants: } 22 \\
\text { Stroke criteria: unclear } \\
\text { Method of stroke diagnosis: not reported } \\
\text { Inclusion criteria: not reported } \\
\text { Exclusion criteria: not reported } \\
\text { Depression criteria: ZDS } \\
\text { Number included in treatment group: } 11 \\
\text { (45.4\% men, mean age } 69.5 \text {, SD } 2.3) \\
\text { Number included in control group: } 11 \text { (72.7\% } \\
\text { men, mean age } 70.4, \text { SD 3.0) }\end{array}$ & $\begin{array}{l}\text { Treatment: trazodone } 300 \mathrm{mg} / \\
\text { day } \\
\text { Control: placebo } \\
\text { Duration: } 30-45 \text { days } \\
\text { Follow-up: unclear }\end{array}$ & 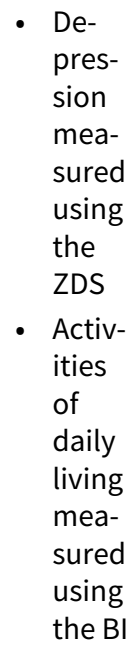 & $\begin{array}{l}\text { Unable } \\
\text { to iso- } \\
\text { late } \\
\text { outcome } \\
\text { data for } \\
\text { non- de- } \\
\text { pressed } \\
\text { partic- } \\
\text { ipants } \\
\text { at ran- } \\
\text { domisa- } \\
\text { tion. }\end{array}$ \\
\hline
\end{tabular}

BI: Barthel Index

CES-D: Center for Epidemiological Studies- Depression

CT: computed tomography

FIM: Functional Independence Measure

GDS: 15-item Geriatric Depression Scale

GHQ-12: 12 item General Health Questionnaire

GHQ-28: 28 item General Health Questionnaire

HADS: Hospital Anxiety and Depression Scale

HDRS: Hamilton Depression Rating Scale

MADRS: Montgomery-Asberg Depression Rating Scale

MRI: magnetic resonance imaging

mRS: modified Rankin Scale

NIHSS: National Institutes of Health Stroke Scale

SD: Standard Deviation

SF-36: 36-item Short Form Questionnaire

SSRI: selective serotonin reuptake inhibitors

ZDS: Zund Depression Scale

Pharmacological, psychological and non-invasive brain stimulation interventions for preventing depression after stroke (Review) 


\section{APPENDICES}

\section{Appendix 1. Search Review: 2008}

\section{Electronic searches}

Cochrane Stroke trial register searched October 2007; Cochrane Anxiety and Neurosis trial register searched February 2008.

We searched the remaining databases in May 2006:

- Cochrane Central Register of Controlled Trials (CENTRAL)

- MEDLINE

- EMBASE

- CINAHL

- Psycinfo

- Applied Science and Technology Plus

- Arts and Humanities Index

- Biological Abstracts

- BIOSIS Previews

- General Science Plus

- Science Citation Index

- Social Sciences Citation Index

- ISI Web of Science

- Dissertations and Theses

The following search strategy with a combination of controlled vocabulary and free text terms for MEDLINE and CINAHL (Ovid), and modified it to suit the other databases.

1. exp cerebrovascular disorders/

2. (stroke\$ or poststroke\$ or cva\$).tw.

3. (cerebrovascular\$ or cerebral vascular).tw.

4. (cerebral or cerebellar or brain $\$$ or vertebrobasilar).tw.

5. (infarct\$ or isch?emi\$ or thrombo $\$$ or emboli\$ or apoplexy).tw.

6. (cerebral or intracerebral or intracranial or brain\$).tw.

7. (haemorrhage or hemorrhage or bleed\$).tw.

8. 4 and 5

9. 6 and 7

10.1 or 2 or 3 or 8 or 9

11.Depression/

12.Depression, involutional/ or Depressive disorder/ or Dysthymic disorder/

13. (depress\$ or dysthymi\$).tw.

14.11 or 12 or 13

15.10 and 14

16. randomized controlled trial.pt.

17.randomized controlled trials/

18. controlled clinical trial.pt.

19.controlled clinical trials/

20.random allocation/

21.double-blind method/

22.single-blind method/

23.clinical trial.pt.

24.exp clinical trials/

25. (clin\$ adj25 trial\$).tw.

26. ((singl\$ or doubl\$ or tripl\$ or trebl\$) adj25 (blind\$ or mask\$)).tw.

27.placebos/

28. placebo\$.tw. 
29. random\$.tw.

30.research design/

31.clinical trial phase ii.pt.

32. clinical trial phase iii.pt.

33.clinical trial phase iv.pt.

34.meta analysis.pt.

35. multicenter study.pt.

36.intervention studies/

37.cross-over studies/

38.meta-analysis/

39.controls.tw.

40.alternate treatment.tw.

41."comparative study"/

42.exp evaluation studies/

43. Follow-up studies/

44.Prospective studies/

45. prospective.tw.

46. (versus or sham or intervention group or comparative stud\$).tw.

47.or/16-46

48.15 and 47

49. limit 48 to human

\section{Additional searches}

We searched the following conference abstracts and proceedings:

- European Stroke Conferences (2000 to 2007)

- Stroke Society of Australasia Annual Scientific Meetings (1999 to 2007)

We also serached the followinh online clinical trials and research registries in August 2007:

- www.strokecenter.org/trials

- www.ClinicalTrials.gov

- www.Clinicalstudyresults.org

- www.anzctr.org.au

\section{Reference lists}

We searched reference lists of relevant studies to identify studies not already included.

\section{Personal communication}

We contacted professional bodies, authors of included studies, and pharmaceutical companies for information on published and unpublished information.

\section{Appendix 2. Search Review 2018: CENTRAL}

Search strategy for CENTRAL, August 2018

\begin{tabular}{|c|c|}
\hline \# & Query \\
\hline \#1 & $\begin{array}{l}\text { [mh “"cerebrovascular disorders"] or [mh "basal ganglia cerebrovascular disease"] or [mh "brain } \\
\text { ischemia"] or [mh "carotid } \\
\text { artery diseases"] or [mh "intracranial arterial diseases"] or [mh "intracranial embolism and throm- } \\
\text { bosis"] or [mh "intracranial } \\
\text { hemorrhages"] or [mh ^stroke] or [mh "brain infarction"] or [mh “"stroke, lacunar"] or [mh ^"va- } \\
\text { sospasm, intracranial”] or }\end{array}$ \\
\hline
\end{tabular}

Pharmacological, psychological and non-invasive brain stimulation interventions for preventing depression after stroke (Review) 
[mh “"vertebral artery dissection"]

\#2

stroke or poststroke or "post-stroke" or cerebrovasc $c^{*}$ or brain next vasc" or cerebral next vasc" or cva* $^{*}$ or apoplex* or SAH:ti,

$\mathrm{ab}, \mathrm{kw}$ (Word variations have been searched)

\#3

(brain* ${ }^{\star}$ or cerebr ${ }^{\star}$ or cerebell ${ }^{\star}$ or intracran ${ }^{\star}$ or intracerebral) near/5 (isch*emi ${ }^{\star}$ or infarct* or thrombo* $^{*}$ or emboli* or occlus*)

:ti,ab,kw (Word variations have been searched)

\#4

(brain* ${ }^{\star}$ crebre ${ }^{\star}$ or cerebell* or intracerebral or intracranial or subarachnoid) near/5 (haemor-

rhage* or hemorrhage* or

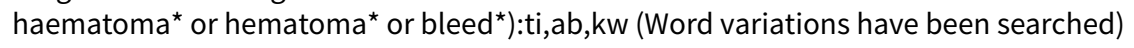

\begin{tabular}{|c|c|}
\hline \#5 & [mh hemiplegia] or [mh paresis] \\
\hline \#6 & hemipleg* or hemipar* or paresis or paretic:ti,ab,kw (Word variations have been searched) \\
\hline \#7 & $\# 1$ or \#2 or \#3 or \#4 or \#5 or \#6 \\
\hline \#8 & $\begin{array}{l}\text { [mh ^ “depressive disorder”] or [mh ^ “depressive disorder, major”] or [mh ^ “depressive disorder, } \\
\text { treatment-resistant”] or [mh } \\
\text { ^"dysthymic disorder”] or [mh ^depression] or [mh “antidepressive agents”] }\end{array}$ \\
\hline \#9 & $\begin{array}{l}\text { depress* or dysthymi*or dysphor*or antidepress*or anti-depress*:ti,ab,kw (Word variations have } \\
\text { been searched) }\end{array}$ \\
\hline \#10 & \#8 or \#9 \\
\hline \#11 & $\# 7$ and \#10 \\
\hline
\end{tabular}

\section{Appendix 3. Search Review 2018: MEDLINE}

Search strategy for MEDLINE, August 2018

1. cerebrovascular disorders/ or exp basal ganglia cerebrovascular disease/ or exp brain ischemia/ or exp carotid artery diseases/ or exp intracranial arterial diseases/ or exp "intracranial embolism and thrombosis"/ or exp intracranial hemorrhages/or stroke/ or exp brain infarction/ or stroke, lacunar/ or vasospasm, intracranial/ or vertebral artery dissection/

2. stroke or poststroke or post-stroke or cerebrovasc $\$$ or brain vasc $\$$ or cerebral vasc $\$$ or cva $\$$ or apoplex\$ or SAH).tw.

3. ((brain\$ or cerebr\$ or cerebell\$ or intracran\$ or intracerebral) adj5 (isch?emi\$ or infarct\$ or thrombo $\$$ or emboli\$ or occlus\$)).tw.

4. ((brain $\$$ or cerebr\$ or cerebell\$ or intracerebral or intracranial or subarachnoid) adj5 (haemorrhage\$ or hemorrhage\$ or haematoma \$ or hematoma or bleed\$)).tw.

5. hemiplegia/ or exp paresis/

6. (hemipleg\$ or hemipar\$ or paresis or paretic).tw.

7. 1 or 2 or 3 or 4 or 5 or 6

8. depressive disorder/ or depressive disorder, major/ or depressive disorder, treatment-resistant/ or dysthymic disorder/ or Depression/ or exp Antidepressive Agents/

9. (depress $\$$ or dysthymi\$ or dysphor $\$$ or antidepress $\$$ or anti-depress $\$$ ).tw.

10.8 or 9

11. Randomized Controlled Trials as Topic/

12.random allocation/

13. Controlled Clinical Trials as Topic/

14.control groups/

15.clinical trials as topic/ or clinical trials, phase i as topic/ or clinical trials, phase ii as topic/ or clinical trials, phase iii as topic/ or clinical trials, phase iv as topic/ 
16.double-blind method/

17.single-blind method/

18.Placebos/

19. placebo effect/

20.cross-over studies/

21. Therapies, Investigational/

22.Drug Evaluation/

23. Research Design/

24. randomized controlled trial.pt.

25.controlled clinical trial.pt.

26. (clinical trial or clinical trial phase i or clinical trial phase ii or clinical trial phase iii or clinical trial phase iv).pt.

27.(random\$ or RCT or RCTs).tw.

28. (controlled adj5 (trial\$ or stud\$)).tw.

29. (clinical\$ adj5 trial\$).tw.

30. ((control or treatment or experiment $\$$ or intervention) adj5 (group $\$$ or subject $\$$ or patient $\$)) . t w$.

31. (quasi-random $\$$ or quasi random $\$$ or pseudo-random $\$$ or pseudo random $\$$ ).tw.

32. ((control or experiment\$ or conservative) adj5 (treatment or therapy or procedure or manage\$)).tw.

33. ((singl\$ or doubl\$ or tripl\$ or trebl\$) adj5 (blind\$ or mask\$)).tw.

34. (cross-over or cross over or crossover).tw.

35. (placebo\$ or sham).tw.

36.trial.ti.

37.(assign\$ or allocat\$).tw.

38.or/11-37

39.7 and 10 and 38

40.exp animals/ not humans.sh.

41.39 not 40

42.limit 41 to $y r=" 2014$-Current"

\section{Appendix 4. Search Review 2018: Embase}

Search strategy for Embase, August 2018

1. cerebrovascular disease/ or basal ganglion hemorrhage/ or exp brain hematoma/ or exp brain hemorrhage/ or exp brain infarction/ or exp brain ischemia/ or exp carotid artery disease/ or cerebral artery disease/ or exp cerebrovascular accident/ or exp intracranial aneurysm/ or exp occlusive cerebrovascular disease/

2. stroke patient/ or stroke unit/

3. (stroke or poststroke or post-stroke or cerebrovasc $\$$ or brain vasc $\$$ or cerebral vasc $\$$ or cva $\$$ or apoplex $\$$ or SAH).tw.

4. ((brain $\$$ or cerebr\$ or cerebell\$ or intracran $\$$ or intracerebral) adj5 (isch?emi\$ or infarct $\$$ or thrombo $\$$ or emboli\$ or occlus\$)).tw.

5. ((brain $\$$ or cerebr\$ or cerebell\$ or intracerebral or intracranial or subarachnoid) adj5 (haemorrhage $\$$ or hemorrhage $\$$ or haematoma $\$$ or hematoma or bleed\$)).tw.

6. hemiparesis/ or hemiplegia/

7. (hemipleg\$ or hemipar\$ or paresis or paretic).tw.

8. 1 or 2 or 3 or 4 or 5 or 6 or 7

9. depression/or agitated depression/or atypical depression/ or dysphoria/ or dysthymia/ or endogenous depression/ or involutional

10.depression/ or late life depression/ or major depression/ or masked depression/ or reactive depression/ or recurrent brief depression/ or treatment resistant depression/ exp antidepressant agent/

11. (depress\$ or dysthymi\$ or dysphor\$ or antidepress\$ or anti-depress\$).tw.

12.9 or 10 or 11

13. Randomized Controlled Trial/ or "randomized controlled trial (topic)"/

14.Randomization/

15.Controlled clinical trial/ or "controlled clinical trial (topic)"/

16.control group/ or controlled study/

17.clinical trial/ or "clinical trial (topic)"/ or phase 1 clinical trial/ or phase 2 clinical trial/ or phase $3 \mathrm{clinical}$ trial/ or phase $4 \mathrm{clinical}$ trial/ 18.Crossover Procedure/ 
19.Double Blind Procedure/

20.Single Blind Procedure/ or triple blind procedure/

21. placebo/ or placebo effect/

22. (random\$ or RCT or RCTs).tw.

23.(controlled adj5 (trial\$ or stud\$)).tw.

24. (clinical\$ adj5 trial\$).tw.

25. ((control or treatment or experiment\$ or intervention) adj5 (group\$ or subject\$ or patient\$)).tw.

26. (quasi-random $\$$ or quasi random\$ or pseudo-random\$ or pseudo random\$).tw.

27. ((control or experiment\$ or conservative) adj5 (treatment or therapy or procedure or manage\$)).tw.

28. ((singl\$ or doubl\$ or tripl\$ or trebl\$) adj5 (blind\$ or mask\$)).tw.

29. (cross-over or cross over or crossover).tw.

30.(placebo\$ or sham).tw.

31.trial.ti.

32.(assign\$ or allocat\$).tw.

33.or/13-32

34.8 and 12 and 33

35. (exp animals/ or exp invertebrate/ or animal experiment/ or animal model/ or animal tissue/ or animal cell/ or nonhuman/) not 36.(human/ or normal human/ or human cell/)

37.34 not 35

\section{Appendix 5. Search Review 2018: PsycINFO}

Search strategy for PsycINFO, August 2018

1. cerebrovascular disorders/ or cerebral hemorrhage/ or exp cerebral ischemia/ or cerebral small vessel disease/ or cerebrovascular accidents/ or subarachnoid hemorrhage/

2. (stroke or poststroke or post-stroke or cerebrovasc $\$$ or brain vasc $\$$ or cerebral vasc $\$$ or cva $\$$ or apoplex $\$$ or $S A H) . t w$.

3. ((brain\$ or cerebr\$ or cerebell\$ or intracran\$ or intracerebral) adj5 (isch?emi\$ or infarct $\$$ or thrombo $\$$ or emboli\$ or occlus\$)).tw.

4. ((brain $\$$ or cerebr\$ or cerebell\$ or intracerebral or intracranial or subarachnoid) adj5 (haemorrhage $\$$ or hemorrhage $\$$ or haematoma \$ or hematoma\$ or bleed\$)).tw.

5. hemiparesis/ or hemiplegia/

6. (hemipleg\$ or hemipar\$ or paresis or paretic).tw.

7. 1 or 2 or 3 or 4 or 5 or 6

8. major depression/ or dysthymic disorder/ or endogenous depression/ or reactive depression/ or recurrent depression/ or treatment resistant depression/ or atypical depression/ or "depression (emotion)"/

9. exp antidepressant drugs/

10.(depress\$ or dysthymi\$ or dysphor\$ or antidepress\$ or anti-depress\$).tw.

11.8 or 9 or 10

12.clinical trials/ or treatment effectiveness evaluation/ or placebo/

13.treatment outcome clinical trial.md.

14. (random\$ or RCT or RCTs).tw.

15.(controlled adj5 (trial\$ or stud\$)).tw.

16. (clinical\$ adj5 trial\$).tw.

17.((control or treatment or experiment\$ or intervention) adj5 (group\$ or subject\$ or patient\$)).tw.

18. (quasi-random $\$$ or quasi random\$ or pseudo-random $\$$ or pseudo random\$).tw.

19. ((control or experiment\$ or conservative) adj5 (treatment or therapy or procedure or manage\$)).tw.

20. ((singl\$ or doubl\$ or tripl\$ or trebl\$) adj5 (blind\$ or mask\$)).tw.

21. (cross-over or cross over or crossover).tw.

22. (placebo\$ or sham).tw.

23.trial.ti.

24. (assign\$ or allocat\$).tw.

25.or/12-24

26.7 and 11 and 25

Pharmacological, psychological and non-invasive brain stimulation interventions for preventing depression after stroke (Review) 


\section{Appendix 6. Search Review 2018: CINAHL}

Search strategy for CINAHL, August 2018

\begin{tabular}{|c|c|}
\hline \# & Query \\
\hline S1 & $\begin{array}{l}\text { (MH“CerebrovascularDisorders”)OR (MH“Basal Ganglia CerebrovascularDisease+”) OR (MH“Carotid } \\
\text { ArteryDiseases+”) } \\
\text { OR (MH “Cerebral Ischemia+”) OR (MH “Cerebral Vasospasm”) OR (MH “Intracranial Arterial Dis- } \\
\text { eases+”) OR (MH } \\
\text { "Intracranial Embolism and Thrombosis”) OR (MH “Intracranial Hemorrhage+") OR (MH “Stroke”) } \\
\text { OR (MH “Vertebral } \\
\text { Artery Dissections”) }\end{array}$ \\
\hline
\end{tabular}

$\mathrm{S} 2$

(MH “Stroke Patients") OR (MH “Stroke Units”)

S3 TI (stroke or poststroke or post-stroke or cerebrovasc* or brain vasc ${ }^{\star}$ or cerebral vasc or cva or apoplex or $\mathrm{SAH}$ ) or $\mathrm{AB}$ (stroke

or poststroke or post-stroke or cerebrovasc* or brain vasc* or cerebral vasc or cva or apoplex or $\mathrm{SAH})$

\begin{tabular}{|c|c|}
\hline S4 & $\begin{array}{l}\text { TI }\left(\text { brain }^{\star} \text { or cerebr }{ }^{\star} \text { or cerebell }{ }^{\star} \text { or intracran* or intracerebral ) or AB ( brain }{ }^{\star} \text { or cerebr }{ }^{\star} \text { or cerebell }\right. \\
\text { or intracran }{ }^{\star} \text { or } \\
\text { intracerebral) }\end{array}$ \\
\hline S5 & $\begin{array}{l}\text { TI (ischemi* or ischaemi* or infarct }{ }^{\star} \text { or thrombo* or emboli* or occlus }{ }^{\star} \text { ) or AB (ischemi* or ischae- } \\
\text { mi }^{\star} \text { or infarct* or thrombo } \\
\text { or emboli* or occlus }{ }^{\star} \text { ) }\end{array}$ \\
\hline S6 & S4 and S5 \\
\hline S7 & $\begin{array}{l}\text { TI (brain* or cerebr }{ }^{\star} \text { or cerebell* or intracerebral or intracranial or subarachnoid) or AB (brain* or } \\
\text { cerebr }{ }^{\star} \text { or cerebell* or } \\
\text { intracerebral or intracranial or subarachnoid) }\end{array}$ \\
\hline S8 & $\begin{array}{l}\text { TI (haemorrhage* or hemorrhage* or haematoma* or hematoma* or bleed }{ }^{\star} \text { ) or AB } \text { (haemorrhage }^{\star} \\
\text { or hemorrhage* or } \\
\text { haematoma* or hematoma* or bleed }{ }^{\star} \text { ) }\end{array}$ \\
\hline S9 & S7 and S8 \\
\hline S10 & (MH “Hemiplegia”) \\
\hline S11 & $\mathrm{TI}$ (hemipleg* or hemipar* or paresis or paretic) or $\mathrm{AB}$ (hemipleg* or hemipar* or paresis or paretic) \\
\hline S12 & $\mathrm{S} 1$ or $\mathrm{S} 2$ or $\mathrm{S} 3$ or $\mathrm{S} 6$ or $\mathrm{S} 9$ or $\mathrm{S} 10$ or $\mathrm{S} 11$ \\
\hline S13 & (MH “Depression”) OR (MH “Depression, Reactive”) OR (MH “Dysthymic Disorder”) \\
\hline S14 & (MH “Antidepressive Agents+”) \\
\hline S15 & $\begin{array}{l}\text { TI (depress* or dysthymi*or dysphor }{ }^{\star} \text { or antidepress }{ }^{\star} \text { or anti-depress }{ }^{\star} \text { ) OR AB (depress }{ }^{\star} \text { or dysthy- } \\
\text { mi*or dysphor }^{\star} \text { or antidepress } \\
\text { or anti-depress }{ }^{\star} \text { ) }\end{array}$ \\
\hline S16 & S13 OR S14 OR S15 \\
\hline
\end{tabular}




\begin{tabular}{|c|c|}
\hline S17 & (MH "Randomized Controlled Trials") or (MH “Random Assignment”) or (MH “Random Sample+”) \\
\hline S18 & (MH "Clinical Trials") or (MH “Intervention Trials”) or (MH “Therapeutic Trials”) \\
\hline S19 & (MH "Double-blind Studies") or (MH “Single-Blind Studies”) or (MH “Triple-Blind Studies”) \\
\hline S20 & (MH "Control (Research)") or (MH “Control Group”) or (MH “Placebos”) or (MH “Placebo Effect”) \\
\hline S21 & (MH “Crossover Design”) OR (MH “Quasi-Experimental Studies”) \\
\hline S22 & PT (clinical trial or randomized controlled trial) \\
\hline S23 & $\mathrm{TI}$ (random* or RCT or RCTs) or AB (random* or RCT or RCTs) \\
\hline S24 & TI (controlled N5 (trial* or stud $\left.\left.{ }^{\star}\right)\right)$ or AB (controlled N5 (trial* or stud $\left.\left.{ }^{\star}\right)\right)$ \\
\hline S25 & TI (clinical* N5 trial* $)$ or AB (clinical* N5 trial*) \\
\hline S26 & $\begin{array}{l}\left.\left.\text { TI ((control or treatment or experiment* or intervention) N5 (group }{ }^{\star} \text { or subject }{ }^{\star} \text { or patient }^{\star}\right)\right) \text { or AB } \\
\text { ((control or treatment } \\
\left.\left.\text { or experiment }{ }^{\star} \text { or intervention) N5 (group } \text { or subject }^{\star} \text { or patient }^{\star}\right)\right)\end{array}$ \\
\hline
\end{tabular}

\begin{tabular}{|c|c|}
\hline S27 & $\begin{array}{l}\left.\left.\text { TI ((control or experiment }{ }^{\star} \text { or conservative) N5 (treatment or therapy or procedure ormanage }{ }^{\star}\right)\right) \text { or } \\
\mathrm{AB}((\text { control or experiment } \\
\left.\left.\text { or conservative) N5 (treatment or therapy or procedure or manage }{ }^{\star}\right)\right)\end{array}$ \\
\hline $\mathrm{S} 28$ & 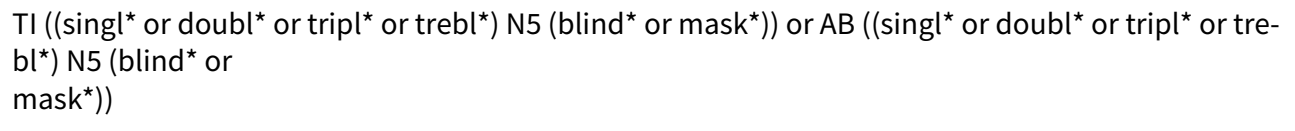 \\
\hline S29 & $\mathrm{TI}$ (cross-over or cross over or crossover) or AB (cross-over or cross over or crossover) \\
\hline S30 & TI (placebo* or sham) or AB (placebo* or sham) \\
\hline S31 & TI trial \\
\hline S32 & $\mathrm{TI}$ (assign* or allocat ${ }^{\star}$ ) or AB (assign ${ }^{\star}$ or allocat ${ }^{\star}$ ) \\
\hline S33 & TI controls or AB controls \\
\hline S34 & $\begin{array}{l}\text { TI (quasi-random* or quasi random* or pseudo-random* or pseudo random }{ }^{\star} \text { ) or AB (quasi-ran- } \\
\text { dom }^{\star} \text { or quasi random }{ }^{\star} \text { or } \\
\text { pseudo-random }{ }^{\star} \text { or pseudo random }{ }^{\star} \text { ) }\end{array}$ \\
\hline S35 & $\begin{array}{l}\text { S } 17 \text { OR S } 18 \text { OR S } 19 \text { OR S } 20 \text { OR S } 21 \text { OR S22 OR S } 23 \text { OR S } 24 \text { OR S } 25 \text { OR S } 26 \text { OR S } 27 \text { OR S } 28 \text { OR S } 29 \text { OR } \\
\text { S30 OR } \\
\text { S31 OR S32 OR S33 OR S34 S36.S12 AND S16 AND S } 35\end{array}$ \\
\hline S36 & S6 AND S16 AND S35 \\
\hline
\end{tabular}

\section{Appendix 7. Search Review 2018: Web of Science}

\section{Search strategy for Web of Science, August 2018}

We searched the following indexes Science Citation Index Expanded (SCI-EXPANDED), Social Sciences Citation Index (SSCI), and Arts \& Humanities Citation Index (A\&HCl) within Web of Science, from January 2002 to August 2018. 


\begin{tabular}{|c|c|}
\hline \# & Query \\
\hline$\# 1$ & $\begin{array}{l}\text { TS=(stroke or post-stroke or post-stroke or cerebrovasc }{ }^{\star} \text { or brain vasc }{ }^{\star} \text { or cerebral vasc }{ }^{\star} \text { or cva* or } \\
\text { apoplex }{ }^{\star} \text { or SAH) }\end{array}$ \\
\hline \#2 & $\begin{array}{l}\text { TS }=\left(\left(\text { brain }^{\star} \text { or cerebr }{ }^{\star} \text { or cerebell }{ }^{\star} \text { or intracran }{ }^{\star} \text { or intracerebral) NEAR/5 (isch\$ } \text { emi }^{\star} \text { or infarct }^{\star} \text { or }\right.\right. \\
\text { thrombo* or emboli* or } \\
\left.\left.\text { occlus }^{\star}\right)\right)\end{array}$ \\
\hline \#3 & $\begin{array}{l}\text { TS=((brain* } \text { or cerebr }^{\star} \text { or cerebell }{ }^{\star} \text { or intracerebral or intracranial or subarachnoid) NEAR/5 (haem- } \\
\text { orrhage } \text { or hemorrhage* }^{\star} \\
\left.\left.\text { or haematoma }^{\star} \text { or hematoma* } \text { or bleed }^{\star}\right)\right)\end{array}$ \\
\hline \#4 & TS=(hemipleg ${ }^{\star}$ or hemipar ${ }^{\star}$ or paresis or paretic or hemineglect or hemi-neglect) \\
\hline \#5 & $\mathrm{TS}=(($ unilateral or spatial or hemi\$spatial or visual) NEAR/5 neglect) \\
\hline \#6 & \#5 OR \#4 OR \#3 OR \#2 OR \#1 \\
\hline$\# 7$ & TS $=\left(\right.$ depress $^{\star}$ or dysthymi*or dysphor ${ }^{\star}$ or antidepress ${ }^{\star}$ or anti-depress $\left.{ }^{\star}\right)$ \\
\hline \#8 & $\mathrm{TS}=\left(\right.$ random ${ }^{\star}$ or RCT or RCTs) \\
\hline \#9 & $\mathrm{TS}=\left(\right.$ controlled NEAR/5 $\left(\right.$ trial $^{\star}$ or stud $\left.\left.{ }^{\star}\right)\right)$ \\
\hline \#10 & $\mathrm{TS}=\left(\right.$ clinical $^{\star}$ NEAR $/ 5$ trial $\left.^{\star}\right)$ \\
\hline \#11 & $\mathrm{TS}=\left(\left(\right.\right.$ control or treatment or experiment ${ }^{\star}$ or intervention) NEAR/5 (group ${ }^{\star}$ or subject $^{\star}$ or patient $\left.\left.^{\star}\right)\right)$ \\
\hline \#12 & TS=(quasi-random* or quasi random* or pseudo-random* or pseudo random*) \\
\hline \#13 & $\begin{array}{l}\text { TS }=\left(\left(\text { control or experiment }{ }^{\star} \text { or conservative) NEAR/5 (treatment or therapy or procedure or man- }\right.\right. \\
\left.\left.\text { age }^{\star}\right)\right)\end{array}$ \\
\hline \#14 & $\mathrm{TS}=\left(\left(\right.\right.$ singl ${ }^{\star}$ or doubl ${ }^{\star}$ or tripl* or trebl*) NEAR/5 $\left(\right.$ blind $^{\star}$ or mask $\left.\left.{ }^{\star}\right)\right)$ \\
\hline \#15 & $\mathrm{TS}=($ cross-over or cross over or crossover) \\
\hline \#16 & $\mathrm{TS}=($ placebo* or sham) \\
\hline \#17 & $\mathrm{Tl}=$ trial \\
\hline \#18 & $\mathrm{TS}=\left(\right.$ assign $^{\star}$ or allocat $\left.{ }^{\star}\right)$ \\
\hline \#19 & $\mathrm{TS}=$ controls \\
\hline \#20 & \#8 OR \#9 OR \#10 OR \#11 OR \#12 OR \#13 OR \#14 OR \#15 OR \#16 OR \#17 OR \#18 or \#19 \\
\hline \#21 & \#6 AND \#7 AND \#20 \\
\hline
\end{tabular}

\section{Appendix 8. Search Review 2018: Other sources \\ Additional searches}

We searched the following conference abstracts and proceedings: 
- European Stroke Conference (2011 to 2018)

- Stroke Society of Australasia Annual Scientific Meetings (2011 to 2017)

- World Stroke Congress (2000 to 2016)

- Asia Pacific Stroke Conference (2011 to 2017)

We also searched online clinical trials and research registers in August 2018

- www.ClinicalTrials.gov (https://clinicaltrials.gov/)

( depression OR low mood) AND ( Brain Infarction OR Intracranial Hemorrhages OR Carotid Artery Diseases OR Brain Ischemia

OR Cerebral Hemorrhage OR Cerebrovascular Disorders OR Stroke )

- WHO International Clinical Trials Registry Platform (https://www.who.int/ictrp/search/en/)

Condition: stroke AND depression OR low mood

Recruitment status is: ALL

Phases are: ALL

Hide synonyms

- 9-52 DEPRESSIVE DISORDERS, BEREAVEMENT, DEPRESSED, DEPRESSED - SYMPTOM, DEPRESSED MOOD, DEPRESSED MOOD (FINDING), DEPRESSED MOOD (PHYSICAL FINDING), DEPRESSED STATE, DEPRESSIVE DIS, DEPRESSIVE DISORDER, DEPRESSIVE DISORDER (DISORDER), DEPRESSIVE DISORDER [DISEASE/FINDING], DEPRESSIVE DISORDER NOS, DEPRESSIVE DISORDER, NOS, DEPRESSIVE DISORDERS, DEPRESSIVE DISORDERS NOS, DEPRESSIVE ILLNESS, DEPRESSIVE NEUROSES, DEPRESSIVE NEUROSIS, DEPRESSIVE STATE, DEPRESSIVE STATENOS,DEPRESSIVE;DISORDER,DEPRESSIVE;NEUROSIS,DEPRESSIVE; STATE,DISORDER,DEPRESSIVE,DISORDER; DEPRESSIVE,DISORDERS,DEPRESSIVE,DYSTHYMIC DISORDER, FEELINGBLUE, FEELINGDOWN, FEELING; DOWN, LOW MOOD, MELANCHOLY, MISERABLE, MOOD DEPRESSED, MOOD DISORDER OF DEPRESSED TYPE, MOOD DISORDER OF DEPRESSED TYPE (DISORDER), MOROSE MOOD, NEUROSES, DEPRESSIVE, NEUROSIS, DEPRESSIVE, NEUROSIS; DEPRESSIVE, PUSH DOWN OR DEPRESS, STATE; DEPRESSIVE, depression - DEPRESSED, DEPRESSED MOOD, DEPRESSED MOOD (FINDING), DEPRESSED MOOD (PHYSICAL FINDING), FEELING BLUE, FEELING DOWN, FEELING;DOWN, MELANCHOLY, MOOD DEPRESSED, MOOD DEPRESSION, MOOD DEPRESSIONS,MOROSEMOOD, low mood - ACCIDENT CEREBROVASCULAR, ACCIDENT; CEREBRAL, ACCIDENT; CEREBROVASCULAR, APOPLEXY, APOPLEXY, CEREBROVASCULAR, APOPLEXY; CEREBRAL, BRAIN ATTACK, BRAIN VASCULAR ACCIDENT, BRAIN VASCULAR ACCIDENTS, CEREBRAL VASCULAR ACCIDENT, CEREBRAL VASCULAR EVENTS, CEREBRAL; ACCIDENT, CEREBRAL; APOPLEXY, CEREBROVASCULAR ACCIDENT, CEREBROVASCULAR ACCIDENT (DISORDER), CEREBROVASCULAR ACCIDENTNOS,CEREBROVASCULAR ACCIDENT, NOS, CEREBROVASCULAR ACCIDENTS, CEREBROVASCULAR APOPLEXY, CEREBROVASCULAR; ACCIDENT, CVA, CVA (CEREBRAL VASCULAR ACCIDENT), CVA (CEREBROVASCULARACCIDENT), CVANOS,CVAS (CEREBROVASCULAR ACCIDENT), NEURO: CEREBROVASCULAR ACCIDENT, VASCULAR ACCIDENT, BRAIN, VASCULAR ACCIDENTS, BRAIN, stroke

\section{WHAT'S NEW}

Date Event Description

13 August $2018 \quad$ New citation required and conclusions have changed

There is very low-certainty evidence that pharmacological interventions may prevent depression and improve mood after stroke compared to placebo.

13 August $2018 \quad$ New search has been performed

New co-authors: SA, KC \& C-FH

New interventions included: combination psychological and pharmacological interventions versus a single intervention, and non-invasive brain stimulation interventions and the review title has been updated accordingly.

The following additional outcomes: health-related quality of life (HRQoL), as measured on scales such as the 36 -item short form questionnaire (SF-36, Ware 1993), proportion reporting dependence in self-care activities of daily living (ADL) on the Modified Rankin Scale (mRS, Rankin 1957) and principal caregiver HRQoL and stress have been removed in this update.

Six new trials, with 417 participants included in the review. A total of 19 trials ( 21 interventions), with 1771 participants, are in- 


$\begin{array}{lll}\text { Date Event Description } & \text { Den }\end{array}$

cluded in the review. Data were available for 12 pharmacological trials (14 interventions) and seven psychological therapy trials.

Covidence was used to collate and screen identified titles and abstracts.

MH extracted additional data from previously included trials.

The searches for the review were completed to 13 August 2018.

\section{HISTORY}

Protocol first published: Issue 3, 2002

Review first published: Issue 2, 2004

\begin{tabular}{|c|c|c|}
\hline Date & Event & Description \\
\hline 18 March 2008 & Amended & Converted to new review format. \\
\hline 30 January 2008 & $\begin{array}{l}\text { New citation required and conclusions } \\
\text { have changed }\end{array}$ & Substantive amendment. \\
\hline 30 January 2008 & New search has been performed & $\begin{array}{l}\text { Two new trials have been added: one pharmacological inter- } \\
\text { vention making a total of } 10 \text { pharmacological trials ( } 12 \text { compar- } \\
\text { isons), and one psychological intervention making a total of four } \\
\text { trials. Seven trials require more information before they can be } \\
\text { assessed for inclusion in the review (down from } 14 \text { in the previ- } \\
\text { ous version). Seven trials appear to meet review inclusion crite- } \\
\text { ria but information is not available in a format suitable for pool- } \\
\text { ing. Four trials are ongoing (up from two in the previous version). }\end{array}$ \\
\hline
\end{tabular}

\section{CONTRIBUTIONS OF AUTHORS}

SA: contributed to writing the review, completed title screening and inclusion/exclusion review, extracted data, performed the metaanalyses and GRADE assessment.

KC: completed title screening and inclusion/exclusion review and data extraction.

$\mathrm{CFH}$ : assisted with obtaining, translating and extracting data from Chinese language studies for the current updated review.

$\mathrm{AH}$ : conceived the idea for the review, contributed to the development, writing, and editing of the protocol, and undertook the work necessary to complete the 2004 and 2008 versions of the review, and edited this update.

$\mathrm{MH}$ : contributed to the development, writing, and editing of the protocol and undertook the work necessary to complete the 2004 and 2008 version of the review, and edited each review update.

\section{DECLARATIONS OF INTEREST}

SA: none known

KC: none known

C-FH: none known

$\mathrm{AH}$ : none known

$\mathrm{MH}$ : none known

Pharmacological, psychological and non-invasive brain stimulation interventions for preventing depression after stroke (Review) 


\section{SOURCES OF SUPPORT}

\section{Internal sources}

- The George Institute for International Health, Australia

\section{External sources}

- Stroke Society of Australasia, Overseas Study Scholarship, Australia

- Academic Unit of Psychiatry, University of Leeds, UK

- Department of Clinical Neurosciences, University of Edinburgh, UK

- Clinical Trials Research Unit, University of Auckland, New Zealand

\section{DIFFERENCES BETWEEN PROTOCOL AND REVIEW}

In this update, we expanded the review to include non-invasive brain stimulation such as 1) transcranial magnetic stimulation or repetitive transcranial magnetic stimulation (TMS or rTMS, where a magnetic 'coil' is placed near the head of the person receiving the treatment without making physical contact); 2) transcranial direct current stimulation (tDCS, where a constant, low current is delivered directly to the brain area of interest via small electrodes); 3) cranial electrotherapy stimulation (CES, where a small, pulsed electric current is applied across a person's head); and 4) magnetic seizure therapy (MST), a type of convulsive therapy that involves replacing the electrical stimulation used in electroconvulsive therapy (ECT) with a rapidly alternating strong magnetic stimulation. We also expanded it to include combination interventions with single interventions.

We have removed the following additional outcomes from this update: health-related quality of life (HRQoL), as measured on scales such as the 36-item short form questionnaire (SF-36); proportion reporting dependence in self-care activities of daily living (ADL) on the Modified Rankin Scale (mRS); and principal caregiver HRQoL and stress, as few trials included these data. Trials with these endpoints are included in other Cochrane Reviews.

\section{N DEX TERMS}

\section{Medical Subject Headings (MeSH)}

Affect; Depression [ ${ }^{*}$ prevention \& control]; Depressive Disorder [ ${ }^{*}$ prevention \& control]; ${ }^{\star}$ Psychotherapy; Randomized Controlled Trials as Topic; Stroke [*psychology]

\section{MeSH check words}

Humans 